\title{
Measurement and Simulation of the Nonlocal Dispersion Tensor in Porous Media
}

\author{
by \\ Mark Warwick Hunter
}

\author{
A thesis \\ submitted to the Victoria University of Wellington \\ in fulfilment of the \\ requirements for the degree of \\ Doctor of Philosophy \\ in Physics.
}

Victoria University of Wellington 2010 



\begin{abstract}
Nuclear Magnetic Resonance (NMR) techniques have been used extensively to characterise dispersion and diffusion in porous media. The completely non-invasive nature of the measurements and the ability to measure opaque samples provide the makings for an excellent tool. Detailed understanding of the microstructure of porous media leads to the ability to model and predict macroscopic effects such as ground water flow, oil extraction, blood perfusion and enable understanding of industrial catalytic reactors.

The range of properties that NMR is capable of measuring is extensive but one particular quantity, the nonlocal dispersion tensor has long been identified as an ideal way to characterise dispersive effects at short time and length scales. The nonlocal dispersion tensor is a quantity that is included in theory proposed by Koch and Brady (1987) to explain nonFickian dispersive behaviour. Demonstrated here is, for the first time, a method to measure the tensor.

Details of the newly developed NMR pulse sequence and the post processing technique required to extract the nonlocal dispersion tensor are given. Successful measurements have been undertaken on model systems such as capillary flow and Couette flow. This enabled direct comparison with analytically calculated quantities and excellent agreement is found, thus verifying the methodology.

A complete set of nonlocal dispersion components has been identified and measured in a model porous medium, in this case a random beadpack of monosized spheres. The new measurements provide the ability to infer and characterise the nature of fluid correlations, particularly at short
\end{abstract}


length scales. In parallel, a simulation suite based on a lattice Boltzmann calculation (implemented by a co-worker Dr Andrew Jackson), has been used to independently generate the same nonlocal components as measured. The simulations have also been used to guide the design of further NMR experiments, to further investigate aspects of the new parameter space that the nonlocal dispersion tensor provides and to explore parts of the parameter space that are inaccessible by NMR. Finally, the methodology was adapted to enable nonlocal dispersion measurements on a 'real' porous medium, a Bentheimer sandstone. 


\section{Acknowledgments}

The number of people I would like to thank is almost too many to list, however I will attempt to acknowledge the support I have enjoyed from a number of people. Invariably during a long project there is a huge number of people that I could mention, I apologise for anyone that I miss.

Firstly my supervisor Professor Sir Paul Callaghan, or as most people know him, Paul. Anyone who has had the privilege of interacting with Paul has had their life enormously enriched. I have enjoyed your company for a number of years now, you are a fantastic scientist, mentor, teacher, New Zealander and friend. Your passion for science and understanding, your appreciation of the arts and enthusiasm for life are all inspirational. Paul, I cannot thank you enough or truly express my admiration for you.

Andrew Jackson is a great friend and co-worker, your support has been fantastic and your input to the project was crucial. I have greatly enjoyed the time we have spent together and I wish you and Eilidh all the best in your endeavours into parenthood. Unfortunately my project moved away from the areas of expertise of my second supervisor Shaun Hendy, but your enthusiasm for science is contagious and I sincerely hope my future research moves into areas where we might work closer together.

I cannot forget the influence of Craig Eccles, my masters supervisor and Henning Klank. Craig and Henning taught me how to be a scientist and for that I am truly grateful. Robin Dykstra has also been a great friend, supporter and co-worker for a number of years, I look forward to that continuing. 
I would like to thank Andrew Coy and the team at Magritek Ltd for giving me the opportunity to think about projects that are completely different to my PhD. I have enjoyed learning how to see things from a slightly different perspective.

I am deeply appreciative of all the friends I have made through my work in the NMR lab. All I can do is say, thank you, I hope you know how much I have enjoyed your friendship, support and willingness to either talk about NMR, or not, as the situation demands. It is hard to think about my PhD without considering the impact you have had. Again, at the risk of missing anyone out, you are; William Holmes, Alexandre Khrapitchev, Melanie Britton, Joe Seymour, Sarah Codd, Maria del Rosario Lopez-Gonzalez, Dave Fairhurst, Antoine Lutti, Holgar Egger, Ocean Mercier, Penny Hubbard, Ying Qiao, Antje Gottwald, Kate Washburn, Simon Rogers, Kirk Feindel, Guillaume Madelin, Jen Brown, Suzanne Fielding, Ed Samulski, Petrik Galvosas, Allan Raudsepp, Bradley Douglass, Margaret Brown, Sarah Dadley, Achim Gädke, Alan Rennie, Manu Pouajen-Blakston, Phillip Luey, Meghan Halse, Ben Parkinson, Nick Grinter, Dave Gilmour, Marcel Nogueira d' Eurydice, Wilfred Kittler and Lauren Burcaw. Particular thanks also to Matthias Meyer for assistance with the simulations in the latter stages of my work.

Outside of the lab there are a number of my friends who have been interested enough to try and learn at least one word from my thesis title. Craig and Alesha, David and Nicola and Daniel and Stephanie, you know you will always be a part of my life. Duncan and Justine, Caleb, Robyn, Paul and Jane, Row and Dane, Warwick and Wendy, Rachael and Richard and Rachel and Richard, I look forward to seeing more of you all postthesis.

The unwavering love and unconditional support from my parents Jeannie and Warwick Hunter is second to none, I love you both with all my heart. The same goes for my extended family Rosie, Rodd, Reuben and Jesse Kingston-Smith, Nick and Christy Hunter and Jeremy Hunter. Al- 
ison and Alan MacRae were always very supportive, particularly Alan who was a consistent encouragement to me. His passing is a huge loss and I would have loved to share this with him.

I have been blessed with a truly wonderful second family, most people refer to them as 'in-laws', thank you for your encouragement and support, Colin and Glenys Wilson, Leanne and Daniel Marshall, Kirsten and Thomas Trollope and Tim Wilson. I would also like to acknowledge Heather and Alan McClean, particularly the Pukawa mentoring sessions from Heather.

In talking about unwavering support, I cannot go without thanking Paul Callaghan once more. The personal and professional support I have had from you Paul is something I will hold on to for the rest of my life.

And how can I possibly express my gratitude to my wife Jillian? I hope you know how I 'fell'. Your love, support, friendship, dependability and understanding have been a blessing in every sense. Without you this thesis would not have been possible. You are a fantastic wife, my best friend and an excellent mother. I love you and Samuel with everything I have. I thank you both from the bottom of my heart. There is a symmetry here, on completing my masters thesis, we got married, becoming a family of two. On completing this thesis our family will become four. The excitement that this gives me far overshadows the joy I have in completing this thesis. And that is saying something. 


\section{Contents}

1 Introduction 1

2 Introduction to Porous Media 5

2.1 Introduction . . . . . . . . . . . . . . . . 5

2.1.1 Definition of a Porous Medium . . . . . . . . . . 6

2.1.2 Mass Transport in Porous Media . . . . . . . . . . . 6

2.2 Properties of Porous Media . . . . . . . . . . . . . 7

2.3 Diffusion and Dispersion . . . . . . . . . . . . . 12

2.3 .1 Fick's Law . . . . . . . . . . . . . . . . . . . 14

2.3 .2 Propagators . . . . . . . . . . . . . . 19

2.3.3 The Peclet Number . . . . . . . . . . . . . . . . . . . 20

2.3.4 Eulerian and Lagrangian . . . . . . . . . . . 23

2.3.5 The Velocity Autocorrelation Function . . . . . . . . . 24

2.4 Nonlocal Dispersion . . . . . . . . . . . . . . . 26

2.4.1 Non-Fickian Dispersion . . . . . . . . . . . 26

2.4 .2 Nonlocality . . . . . . . . . . . . . . . 26

2.4 .3 Koch and Brady . . . . . . . . . . . . . . . 27

2.4 .4 Measurement of $\mathbf{D}^{N L} \ldots \ldots \ldots \ldots$

2.5 Conclusions . . . . . . . . . . . . . . . . 31

3 Nuclear Magnetic Resonance 33

3.1 Introduction . . . . . . . . . . . . . . . 33

3.2 The Semi-Classical Model . . . . . . . . . . . . . . . . 34 
3.2.1 Quantum Mechanical Background . . . . . . . . . . 34

3.2.2 Bulk Magnetisation . . . . . . . . . . . . . . . . . 36

3.2 .3 The Rotating Frame . . . . . . . . . . . . . . . . 37

3.2.4 The Bloch Equations . . . . . . . . . . . . . . . 38

3.2.5 Excitation and Detection . . . . . . . . . . . . . . 40

3.2 .6 Relaxation . . . . . . . . . . . . . . . . . . . 42

3.3 Spatial Encoding . . . . . . . . . . . . . . . . . 45

3.3.1 One Dimensional Imaging . . . . . . . . . . . . . 46

3.3.2 Displacement Encoding . . . . . . . . . . . . . . 50

3.3.3 Low-q Approximation . . . . . . . . . . . . . . . . 53

3.3.4 Stimulated Echo . . . . . . . . . . . . . . . . . 55

3.3.5 Double PGSTE . . . . . . . . . . . . . . . . . . . . . . 59

3.3.6 Bi Polar PGSE . . . . . . . . . . . . . . . . . . . . . . . 62

3.4 Dispersion and NMR . . . . . . . . . . . . . . . 62

3.4.1 Ensemble Averaging . . . . . . . . . . . . . . 65

3.5 Technical Aspects of Applying Gradients . . . . . . . . . 65

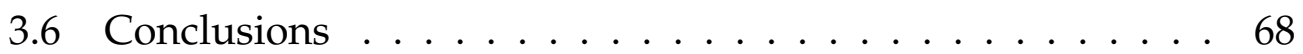

4 Simulation of Dispersion in Porous Media 69

4.1 Introduction . . . . . . . . . . . . . . . . . . 69

4.2 The Simulation Suite . . . . . . . . . . . . . . . . . 70

4.2.1 Lattice Boltzmann Calculation of the Flow Field . . . 70

4.2.2 Beadpack and Lattice Generation . . . . . . . . . . 73

4.2.3 Tracer Particle Simulation . . . . . . . . . . . . . . . 77

4.2.4 Statistical Calculation of Parameters . . . . . . . . . 81

4.2.5 Sample Results . . . . . . . . . . . . . . . . . 81

4.3 Lattice Boltzmann, Dispersion and NMR . . . . . . . . 83

4.4 Conclusions . . . . . . . . . . . . . . . . 86

$5 \quad$ NMR for Nonlocal Dispersion $\quad 87$

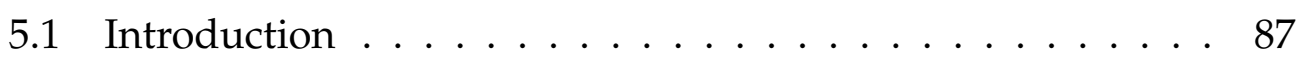

5.2 The Nonlocal Dispersion Tensor _ . . . . . . . . . . . 88 
5.3 NMR Implementation $\ldots \ldots \ldots$

5.3.1 The Pulse Sequence . . . . . . . . . . . . . . . . . . . . 88

5.3.2 The NMR Signal . . . . . . . . . . . . . . . . . . . . 91

5.3.3 The Low- $q$ Limit . . . . . . . . . . . . . . . . . . . . 92

5.3 .4 Extracting $\mathrm{D}^{N L} \ldots \ldots \ldots \ldots \ldots$

5.4 Experimental Method . . . . . . . . . . . . . . . . . . . 95

5.4 .1 Pulse Sequence . . . . . . . . . . . . . . . . . . . . 96

5.4 .2 Post Processing . . . . . . . . . . . . . . . . . . . . . 100

5.4.3 General Experimental Considerations . . . . . . . . 106

5.5 Echo Attenuation Simulation . . . . . . . . . . . . . . 107

5.6 Limits of Double-PGSTE Encoding . . . . . . . . . . . . . 110

5.6.1 Velocity Autocorrelation Function . . . . . . . . . . 112

5.6 .2 Second Moment of Velocity . . . . . . . . . . . . . 113

5.7 Double PGSTE and Asymptotic Conditions . . . . . . . . . 115

5.8 Conclusions . . . . . . . . . . . . . . . . . . . . . 116

6 Model Systems for Nonlocal Dispersion 119

6.1 Introduction . . . . . . . . . . . . . . . . . . . . . . 119

6.2 Taylor Flow in a Capillary . . . . . . . . . . . . . . . 120

6.2.1 Calculation of Nonlocal Dispersion in Capillary Flow 121

6.2.2 Inferring Properties of the Flow . . . . . . . . . . 127

6.2.3 Simulation of Nonlocal Dispersion in Capillary Flow 128

6.2.4 Experimental Measurement of Nonlocal Dispersion in Capillary Flow . . . . . . . . . . . . . 133

6.2.5 Further Improvements . . . . . . . . . . . . . . . 136

6.3 Taylor-Couette Flow . . . . . . . . . . . . . . . . . . 141

6.3.1 Calculation of Nonlocal Dispersion . . . . . . . . 141

6.3.2 Experimental Results . . . . . . . . . . . . . . . . 144

6.4 Conclusions . . . . . . . . . . . . . . . . . . . . . . 148

7 Nonlocal Dispersion in a Porous Medium 149

7.1 Introduction . . . . . . . . . . . . . . . . . . . . . . . . 149 
7.2 Experimental Method . . . . . . . . . . . . . . . . . . 150

7.3 Lattice Boltzmann Simulation . . . . . . . . . . . . . . . 151

7.4 Dimensionality of the Nonlocal Dispersion Tensor . . . . . 151

7.5 Experiment and Simulation Results . . . . . . . . . . . . 152

7.5.1 Definition of Peclet Number . . . . . . . . . . . . . 152

7.5.2 Propagator Measurement . . . . . . . . . . . . 153

7.5.3 $\mathrm{D}^{N L}$ Components from Simulation and Experiments 155

7.5.4 Analysis of Longitudinal Displacement Encodings . . 157

7.5.5 Analysis of Transverse Displacement Encodings . . . 160

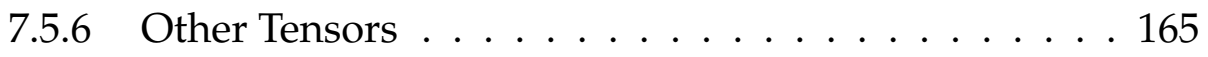

7.6 Conclusions . . . . . . . . . . . . . . . . . . . . 167

8 Higher Dimensional Encodings 173

8.1 Introduction . . . . . . . . . . . . . . . . 173

8.2 Dimensionality . . . . . . . . . . . . . . . . 174

8.3 Simulation . . . . . . . . . . . . . . . . 176

8.4 Visualisation . . . . . . . . . . . . . . . . . 176

8.5 The Components . . . . . . . . . . . . . . . . . 177

8.5.1 Longitudinal Correlations . . . . . . . . . . . . 177

8.5.2 Transverse Correlations . . . . . . . . . . . . . . . . 179

8.5.3 Transverse-Longitudinal Correlations . . . . . . . . 183

8.5.4 Transverse-Transverse Correlations . . . . . . . . 186

8.6 Conclusions . . . . . . . . . . . . . . . . 188

9 Nonlocal Dispersion in a Rock Core 191

9.1 Introduction . . . . . . . . . . . . . . . . . . . . . . . 191

9.2 Experimental Setup . . . . . . . . . . . . . . . . . . . . 192

9.2 .1 Flow Cell . . . . . . . . . . . . . . . . . . . . . . . . . . 192

9.2 .2 Flow Stability . . . . . . . . . . . . . . . . . . . 193

9.2 .3 Internal Gradients . . . . . . . . . . . . . . . . . 193

9.2.4 Measured Components _. . . . . . . . . . . . . 196

9.3 Nonlocal Components . . . . . . . . . . . . . . . . . . . 196 
9.3.1 Longitudinal Correlations . . . . . . . . . . . . . . 196

9.3.2 Transverse Correlations . . . . . . . . . . . . . . . 197

9.4 Conclusions . . . . . . . . . . . . . . . . . . 199

10 Conclusions 203

10.1 The Work Contained in this Thesis . . . . . . . . . . . . . . . 203

10.1.1 A New Measurement Technique . . . . . . . . . . . 203

10.1.2 Analysis of Nonlocal Dispersion . . . . . . . . . . . 205

10.2 Future Work . . . . . . . . . . . . . . . . . . . 206

10.2.1 Experimental Developments . . . . . . . . . . 206

10.2.2 New Systems . . . . . . . . . . . . . . . . 207

10.2.3 Nonlocal Theory of Koch and Brady . . . . . . . . . . 208

10.3 Closing Remarks . . . . . . . . . . . . . . . . 208 


\section{Chapter 1}

\section{Introduction}

Our interactions with porous media encompass a wide range of situations from perhaps the most visible; a kitchen sponge soaking up a spilt drink, to the large scale transport of ground water. The movement of fluid through porous media occurs in a range of systems such as blood flow through organs, nutrient transport in plants, domestic and industrial filtration processes and large scale catalytic reactors. This wide range of applications attracts interest from a range of disciplines.

Dispersion in porous media characterises the way the molecules, initially adjacent, migrate apart due to diffusive, velocity gradient or turbulent effects. These processes have been studied by a range of techniques and there are many standard texts on the physics of flow through porous media [1-3]. The use of Nuclear Magnetic Resonance (NMR) in measuring dispersion and characterising porous media is a research field in itself. The primary advantage of NMR is the ability to probe opaque systems in a non-invasive, non-destructive manner. A series of international conferences over the last 20 years showcases the state-of-the-art in the research of magnetic resonance applications in porous media [4-12].

One quantity of primary importance in fluid dynamics is The Nonlocal Dispersion Tensor. This tensor forms part of a theory proposed by Koch and Brady in 1987 [13] as a means to explain dispersive observations not 
predicted by Fick's laws. The nonlocal dispersion tensor is a quantity that is well defined at short distances and times and has been adopted by the NMR community as potentially an ideal measurement to characterise dispersion [14-24].

This thesis concerns itself with the development of a technique to measure the nonlocal dispersion tensor, and having done that, provide an interpretation for the measurements. Chapters 2-4 form the introduction, 5-9 are the original findings and the dissertation is concluded in chapter 10.

\section{Chapter 2}

Here the basic language of porous media and mass transport in porous media is given. Various NMR methods for characterisation are introduced and briefly discussed. Diffusion and dispersion are treated in some detail, including Fick's law which leads to the need for a nonlocal description.

\section{Chapter 3}

A description of Nuclear Magnetic Resonance is given, heavily skewed towards the techniques used in the remainder of the thesis. These techniques are known as Pulsed Gradient Spin Echo (PGSE) measurements and are essential for measuring particle displacements which are the essence of dispersion. A brief literature review of dispersion measurements and measurement on porous media with NMR is given.

\section{Chapter 4}

An introduction to the simulation suite is given here. It should be noted that the programming of the suite was not the work of the author. A coworker, Dr Andy Jackson, performed the bulk of the programming. The introduction given draws from a submitted paper, containing work that is independent of this thesis, in which Dr Jackson is first author. However the use of the suite and interpretation form many of the original findings 
of the thesis and as such, an introduction is relevant. A brief literature review of NMR and simulated dispersion is given here.

\section{Chapter 5}

This chapter marks the beginning of the original work. The development of the NMR methodology for extracting the nonlocal dispersion tensor is contained here. The details of the NMR pulse sequence, post processing and challenges are given. This chapter is an expansion on the publication "PGSE NMR measurement of the nonlocal dispersion tensor for flow in porous media, M.W. Hunter, A.N. Jackson and P.T. Callaghan. Journal of Magnetic Resonance, Volume 204(1) 2010".

\section{Chapter 6}

The new methodology is applied to model systems in this chapter. Comparison with numerically calculated quantities were made to verify the experimental measurements. The highlights of this chapter appeared as "NMR Measurement of Nonlocal Dispersion in Complex Flows, M.W. Hunter and P.T. Callaghan, Physical Review Letters 99 210602, 2007".

\section{Chapter 7}

Having successfully developed the methodology, a full set of nonlocal dispersion measurements were made on a model porous medium, in this case, a pack of monosized beads. The measured quantities were compared with simulations. The results and interpretations were published as "Nuclear magnetic resonance and lattice Boltzmann simulation of the nonlocal dispersion tensor, M.W. Hunter, A.N. Jackson and P.T. Callaghan Physics of Fluids, 22027101 2010". 


\section{Chapter 8}

The measurements in Chapter 7 suggested a higher dimensional extension that was unaccessible experimentally. The simulation suite is ideal for investigating such aspects of the parameter space. Here, nonlocal dispersion components resolved in three-dimensional displacement space, as a function of time, were investigated.

\section{Chapter 9}

The measurement technique was further adapted to enable experiments on a 'real' porous medium, in this case a Bentheimer rock core. The measurements in chapter 7 suggested that information could still be obtained from an incomplete set of measurements. Despite experimental difficulties, a selection of nonlocal components were measured.

\section{Chapter 10}

A summary of the findings is given. Ideas on suggested technical improvements to the experimental technique are summarised. These improvements could greatly increase the applicability of the technique. Suggestions are made for other dispersive systems where the nonlocal dispersion could be measured. 


\section{Chapter 2}

\section{Introduction to Porous Media}

\subsection{Introduction}

The study of mass transport in porous media, and study of porous media in general, is motivated by the desire to understand and predict macroscopic properties from a deeper understanding and knowledge of the microscopic properties. Macroscopic properties are directly related to 'realworld' effects, such as filtration, predicting ground water flow, designing chemical reactors and the increasing of, or predicting, the economics of oil recovery.

Here we introduce some of the relevant details of porous media and in particular the use of NMR in characterisation. Nuclear magnetic resonance has proven to be invaluable as a tool to characterise porous media, an inherent advantage being the ability to perform measurements on opaque samples. The length scales and time scales associated with NMR measurements are, in general, coincident with the scales of interest in porous media. Almost exclusively the measurements are performed on some liquid or gas that has permeated the pore space and, the use of a fluid as a probe of the material makes this method appropriate to the direct study of the pore space and mass transport. However, the method can also provide an indirect means of studying the structure of the solid 
matrix.

Some basic definitions and concepts of porous media and mass transport will be introduced with a focus on those properties that can be quantified by NMR. Some quantities, such as porosity, are directly related to the medium, while others, such as 'pore size distribution' are closely related to the measurement technique used and as such, the NMR methods used will be introduced in parallel. The role of PGSE NMR will be discussed in the measurement of mass transport and dispersion in porous media followed by a discussion of nonlocal dispersion and a brief introduction to nonlocal theories.

For a thorough treatment on flow in porous media the reader is referred to standard texts $[2 ; 25]$ and general reviews of the use of NMR to characterise porous media are given in references [26;27].

\subsubsection{Definition of a Porous Medium}

A porous medium is a material or region that consists of two or more phases. One of which is solid, called the solid matrix, and the remaining part known as the void space or pore space, can be occupied by a combination of fluid and gas. In real porous media the solid matrix is continuous (although isolated islands of the matrix can exist in simulated porous media). The void space is typically continuous and multiply interconnected although 'dead-end' pores and 'closed' pores can exist. If a medium contains 'closed' pores they are typically treated as part of the solid matrix since no interaction with the bulk void space is possible. Examples of porous media include, wood, lung and kidney tissue, foams and the media studied here; rocks and random packings of monosized spheres.

\subsubsection{Mass Transport in Porous Media}

Nuclear magnetic resonance provides an almost ideal mechanism for studying the behaviour of the spins in the liquid and/or gaseous phases con- 
tained in the pore space of a porous medium. The labelling and subsequent measurement is completely non-invasive and the measurement of fluid in opaque samples is possible. For systems where there is no pressure gradient, and hence no bulk mass transport, pulsed gradient spin echo (PGSE) NMR provides an effective method for measuring the ensemble averaged 'mean-squared-displacement' and hence diffusion. This method, coupled with the known bulk diffusion of the chosen liquid can provide detailed structure of the macro and microscopic properties of the medium. Relaxation measurements are also used to characterise the pore space.

In systems where a pressure gradient is present, and hence a bulk flow, the measurement of the 'mean-squared-displacement' and hence the dispersion, is still important. Such a pressure gradient creates a mechanism for mass transfer and so much emphasis is placed on the study of tracer particles moving through pores, at the microscopic scale, and predicting quantities like 'asymptotic dispersion' [2] and 'residence time distributions' [28].

\subsection{Properties of Porous Media}

The porosity $\phi$ of a porous medium is the ratio of the pore space to the total volume. For a random pack of mono-sized spheres this is typically around 0.4 , rock cores have a porosity typically around 0.15 to 0.3 , depending on their composition. Foams and sponges might have porosities over 0.9.

A representative elementary volume (REV) is the smallest volume from which macroscopic properties can be derived. Such a volume would be able to provide a statistically relevant description of the void space and the solid matrix. Following [2] we describe a definition in terms of the porosity. First we start with an arbitrary shaped volume $\Delta U$, much larger than a pore, and calculate its porosity. As this volume is continuously reduced in size the calculated porosity will be relatively constant (for a homoge- 


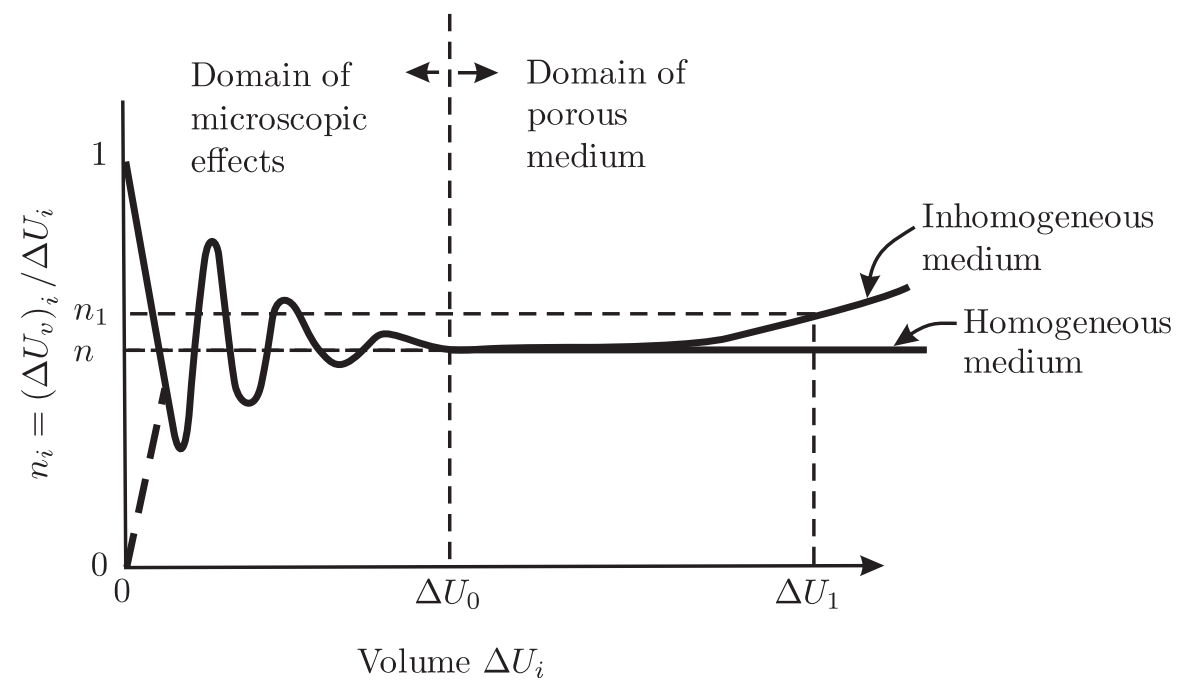

Figure 2.1: Describing the representative elementary volume in terms of porosity, reproduced from [2]. In a homogeneous medium the volume $\Delta U_{0}$ is the smallest volume that provides a measure of porosity that is not dominated by microscopic effects, this provides a measure of a REV. Such a quantity can be difficult to define in a heterogeneous medium. 
nous medium) and will then begin to fluctuate as the dimensions of $\Delta U$ become comparable with the pore size (see figure 2.1). The representative elementary volume is the smallest volume before large fluctuations occur. Inhomogeneous, or heterogenous, media will be characterised by an absence of, or a gradually varying, plateau as in figure 2.1. Model porous media constructed from a random arrangement of mono-sized spheres are thought of as homogenous. Sandstones are described as heterogeneous and carbonates as 'very heterogeneous' [29]. Grossly heterogenous media, such as large aquifers, often show continuum behaviour at a range of length scales. For such a material the graph in figure 2.1 might show a number of plateaus at vastly different $\Delta U_{i}$, and differing values of $n_{i}$. These media are referred to as 'hierarchical' [30].

Heterogeneity and REVs can be defined in terms of other statistical properties [2]. Figure 2.2 shows an example of a homogeneous medium and a heterogenous medium in terms of a 'grain-size distribution'. In each porous medium the solid matrix is described as a monolayer of consolidated grains.

Closely related to a measure of heterogeneity is the measure of a 'poresize distribution'. This is not a well defined quantity since it is difficult to define a pore by a single radius [1], and even the very definition of a single 'pore' is not straight forward. One such explanation states [25], "While a completely general definition of a pore is probably not possible, a "pore" is defined as a portion of pore space bounded by solid surfaces and by planes erected where the hydraulic radius of the pore space exhibits minima". Several methods are used to determine the pore size distribution of a medium, each method effectively defining a pore size according to the nature of the measurement.

For media with a relatively homogeneous structure, a characteristic length can be defined. This refers to some length associated with a REV or a well-defined structural element. For a random pack of monosized spheres the characteristic length is sometimes taken as the bead diameter, $d_{b e a d}$, or 

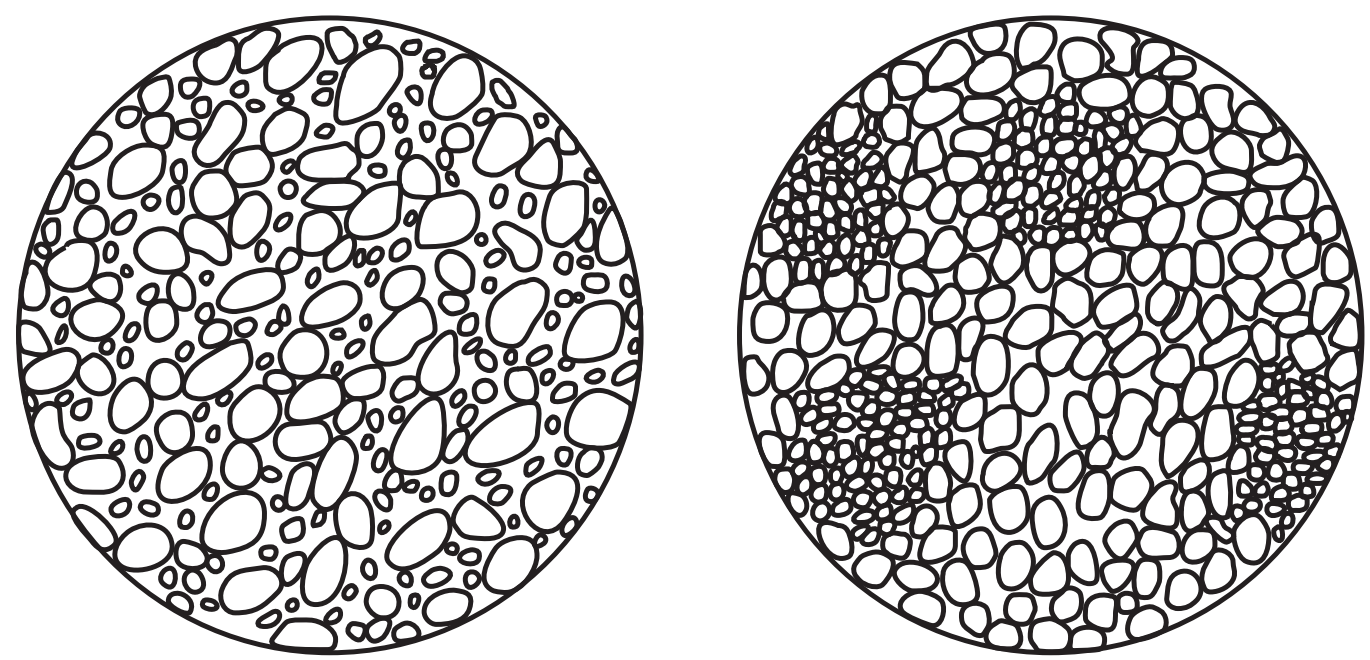

Figure 2.2: An example of a homogeneous porous media (a) and a heterogeneous porous media (b) shown as a monolayer. Reproduced from reference [31]

more often [14] as $d_{\text {bead }} \phi /(1-\phi)$. Heterogeneous and hierarchial media are characterised by a multiplicity of length scales [30; 32].

The ability to characterise pore-size distributions using NMR was first noted in 1979 by Brownstein and Tarr [33]. Here the modes of a multiexponential decay of a measured CPMG decay (see section 3.2.6) were related to the sizes of biological cells, due to surface interactions of diffusing spins. In contrast to the pore space of a porous medium, the individual cells have a well defined size making a pore-size distribution reasonably well defined. The linear relationship between the distribution of exponential decays and the distribution of surface-to-volume ratios of a pore space was noted by Kleinberg et al [34]. In the NMR community the distribution of surface-to-volume ratios has come to mean 'pore size distribution' [35]. Such a NMR measurement is valid for situations where particles do not, in the relaxation measurement time, diffuse to regions where the local surface-to-volume ratio is significantly different.

Comparisons of different measurements of pore size distributions were performed in [36]. Recently, Arns [37] has used micro-X-ray-CT to mea- 
sure the complete fine structure of porous media such as sandstones and carbonate rock. A digitised version of the medium can then be used to simulate the response from NMR experiments. The simulated response can then be used to estimate pore-size-distributions and compared directly to quantities derived directly from the image.

Measurements of the effective diffusion coefficient $D(t)$ (see section 2.3) have also proven to be a useful tool for characterising the surface-to-volume ratio. For longer encoding times, the diffusion of tracer particles is restricting due to the porous medium. Mitra et al. [38] have shown that, independent of any surface relaxation [39], the time dependent diffusion coefficient for short times can be written as

$$
\frac{D(t)}{D_{0}}=\lim _{t \rightarrow 0} 1-\frac{4}{3 n \sqrt{\pi}} \frac{S}{V} \sqrt{D_{0} t}+O\left(D_{0} t\right),
$$

where $n$ is the number of dimensions in which $D(t)$ is measured.

Liaw et al. [40] have performed measurements on a range of model and real porous media. Using an analysis of $T_{1}$ relaxation (see section 3.2.4) and time dependent diffusion measurements, volume-to-surface distribution and pore-size distributions were made. Comparisons to standard techniques, mercury porosimetry and thin-section analysis, were made. Equation 2.1, in the long time limit, also gives the tortuosity, $\Upsilon$, of a porous medium. The tortuosity of a hydrocarbon reservoir provides important information regarding its economic potential. The tortuosity is the ratio of the average path length in the medium squared to the average free path length squared [2]. For this reason the diffusion coefficient provides a good measure. In the limit of long time for equation 2.1 we have [35]

$$
\frac{1}{\Upsilon}=\frac{D_{\infty}}{D_{0}}
$$

For this to be a meaningful measurement, the limiting time must be long enough so that the tracer particles have sampled a large region of the pore space. It was also noted that relaxation time measurements of pore size 
distributions and PGSE measurements of tortuosity are mutually exclusive [35].

In experimental measurements where the limiting value of $D_{\infty}$ is not observed, the observation of $D(t)$ can be used to predict an asymptotic value. An approximation known as Archie's law gives $\frac{D_{\infty}}{D_{0}}=\phi$. The tortuosity can also be written as

$$
\Upsilon=\frac{1}{F \phi},
$$

where $F$ is the electrical formation factor. This is given as the inverse ratio of the conductivity of a pore space filled with an electrolyte, to the the bulk conductivity of the electrolyte.

The permeability, $k$, of a porous medium quantifies the conductivity of mass transport. In analogy to Newton's law of viscosity we write Darcy's law as

$$
Q=\frac{k A}{\mu} \frac{\Delta P}{L},
$$

where the dimensions of the sample are given by the length $L$ and the cross-sectional area (including the solid and the void space) $A$. $Q$ is the volumetric flow rate, the viscosity of the liquid is given by $\mu, \Delta P$ is the pressure drop across the length $L$ and the permeability is $k$. In some cases the permeability of a porous medium can be predicted from knowledge of the microstructure [41].

\subsection{Diffusion and Dispersion}

The phenomenon of diffusion is often studied in the context of the response of a solute to a concentration gradient. Here we will be primarily concerned with the time evolution of particle separation under equilibrium conditions. Einstein has shown that in the absence of a concentration gradient particles will undergo random fluctuations in speed and position 

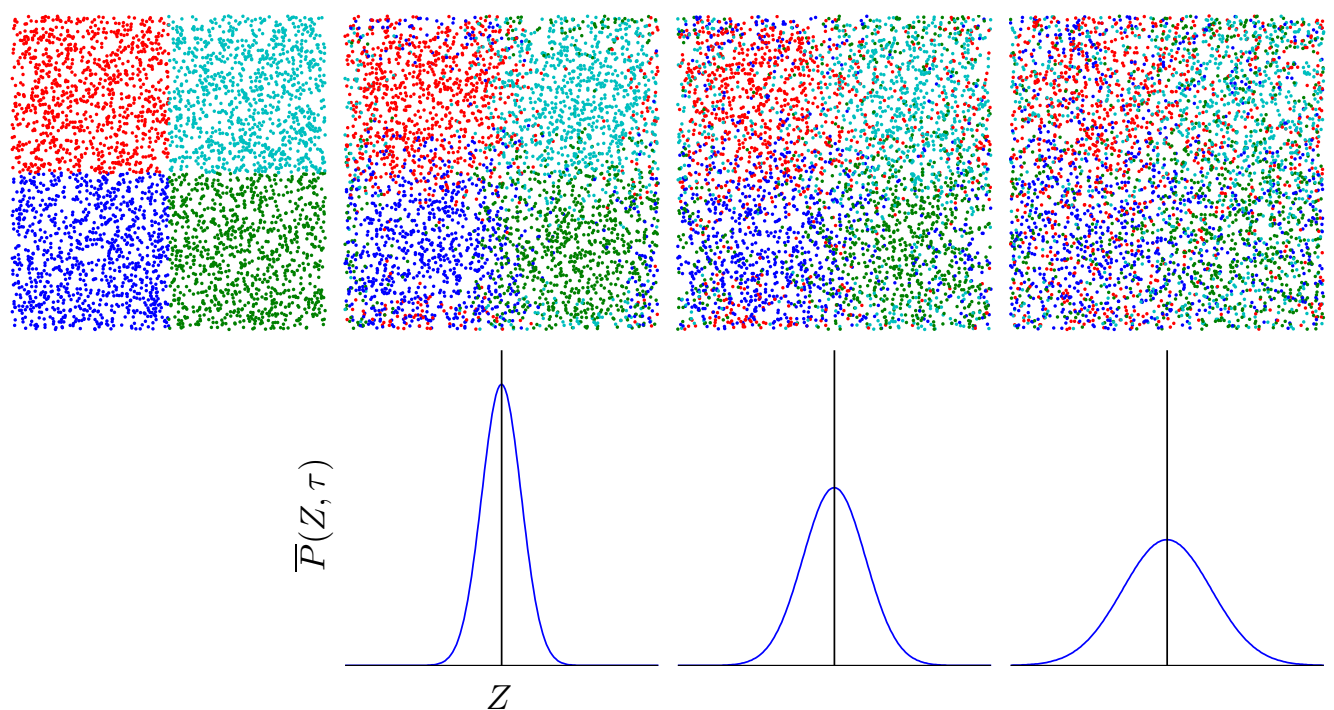

Figure 2.3: The free diffusion of tracer particles are shown at evolving times. The different colours are labels only and the boundaries are periodic. The average propagators, $\bar{P}(Z, \tau)$ describe the probability of particles moving a distance $Z$ in a time $\tau$.

that will lead, statistically, to a well defined displacement as a function of time. In the previous section it was noted that the measurement of the time-dependent diffusion coefficient can provide a method for characterising porous media. Here we will look closer at the definition of this quantity.

The separation of initially adjacent particles for flow in a porous medium is governed by the stochastic process of molecular diffusion and the properties of the underlaying flow field. Most porous media have a sufficiently complicated pore space to make standard Navier-Stokes calculations of the flow field impractical. Numerical calculations for relatively simple, small scale porous media have recently become widespread using techniques such as computational fluid dynamics [42-44] and as detailed in chapter 4, lattice Boltzmann simulations. Knowledge of the flow 
field and diffusive properties enables one to make predictions of the observed macroscopic transport properties such as residence time distributions, propagators, moments, velocity autocorrelation functions and dispersion coefficients. Some of these quantities will be introduced in the following sections. In general these quantities can be predicted by Fick's law, however in section 2.4 we will introduce non-Fickian behaviour and hence the need for the nonlocal dispersion tensor, the topic of this thesis.

\subsubsection{Fick's Law}

\section{Diffusion}

Fick's law allows one to predict the particular concentration $C$ at a time after the introduction of a concentration gradient. Beginning with the continuity equation we have,

$$
\frac{\partial C}{\partial t}-\nabla \cdot \mathbf{j}=\rho,
$$

where $\mathbf{j}$ is the flux density and $\rho$ describes the source of tracer particles. In the absence of a source we have

$$
\frac{\partial C}{\partial t}-\nabla \cdot \mathbf{j}=0
$$

The flux density can be approximated by Fick's law [45], that says the flux is proportional to the gradient of the concentration.

$$
\mathbf{j}=-D \nabla C,
$$

where $D$ is the diffusion coefficient. This can then be substituted into equation 2.5 giving the diffusion equation, or sometimes called Fick's second law

$$
\frac{\partial C(\mathbf{r}, t)}{\partial t}=D \nabla^{2} C(\mathbf{r}, t)
$$



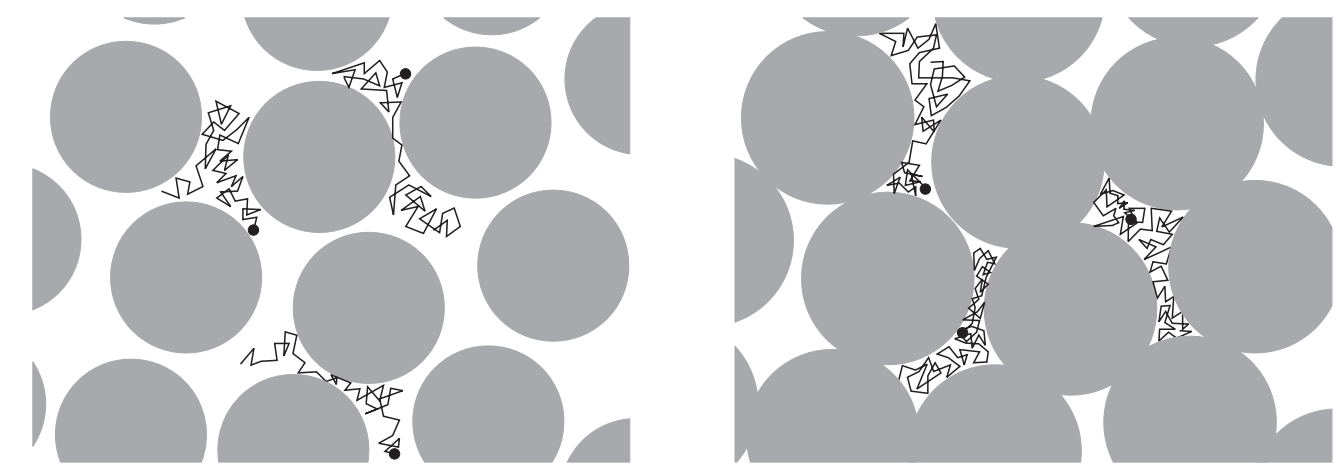

(a)

(b)

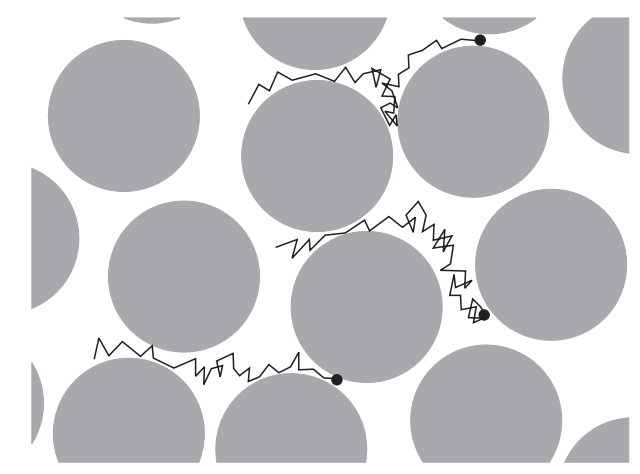

$(c)$

$\langle v\rangle$

Figure 2.4: Panel (a) shows diffusion in a two-dimensional porous medium. Here the diffusion will be restricted but in the long time limit $\sigma^{2} \propto t$, as shown in figure 2.5(a). Panel (b) shows the tracer paths for the same porous medium with larger, consolidated grains resulting in a closed restricted diffusion. The asymptotic mean-squared displacement will be limited by the size of the closed pores. This is shown in figure 2.5(b). Panel (c) shows the dispersion of tracers under flow and diffusion. The growth of the mean squared displacement will also be proportional to time and at a greater rate than free diffusion, as shown in figure 2.5(c). 

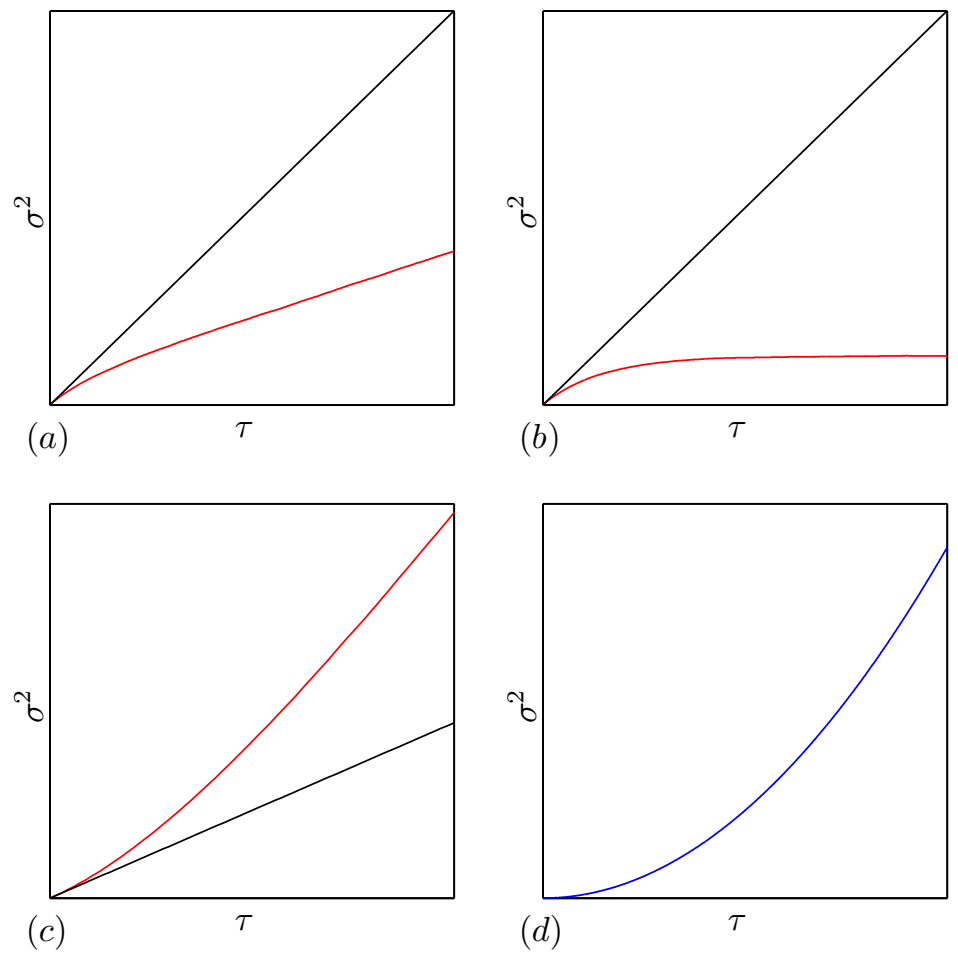

Figure 2.5: Four examples of mean squared displacement as a function of time. Panel (a) shows pure diffusion (black) and restricted open diffusion (red), the slope asymptotes to a value less than that of free diffusion. Panel (b) shows free diffusion (black) and restricted closed diffusion where tracers are confined in a pore, the slope will asymptote to zero. Panel (c) shows free diffusion (black) and dispersion due to diffusion in a laminar velocity field (red), the slope will asymptote to a value greater than that of free diffusion. In all of the cases in (a-c) the initial slope of $\sigma^{2}$ is the same as that of free diffusion. Panel (d) shows the mean squared displacement for laminar flow in the absence of diffusion. No asymptote is reached. 
Consistent with equation 2.8 the usual definition of the self diffusion coefficient $D$ is

$$
\mathbf{D}=\frac{\boldsymbol{\sigma}^{2}}{2 n t}
$$

where $\boldsymbol{\sigma}^{2}$ is the mean squared displacement $\left\langle(\mathbf{r}(t)-\mathbf{r}(0))^{2}\right\rangle$ and $n$ is the number of dimensions in which the displacement is measured. Formally the mean-squared displacement is an outer product, resulting in a diffusion tensor. This tensor is often spherical and characterised by a scalar, diffusion coefficient, $D_{0}$ [46]. Equation 2.8 has been formulated as a response to a change in concentration, but it also applies to the random motions of particles in thermal equilibrium. This self diffusion is shown in figure 2.3.

For fully closed systems, such as diffusion inside spheres, the maximum of the mean squared displacement $\sigma^{2}$ will be bound by the size of the sphere. In restricted open media the growth of the mean squared displacement will be a function of time. This allows for a definition of one-dimensional time-dependent diffusion as

$$
D(t)=\frac{\sigma^{2}(t)}{2 t}
$$

The evolution of this quantity is a consequence of the particle's self diffusion being impeded by the structure of the medium, thus providing information regarding the surface-to-volume ratio and the tortuosity [47]. Figure 2.5(a) shows the growth of $\sigma^{2}$ (in one-dimension) as a function of time for free diffusion (black) and restricted open diffusion (red). At long times the growth of $\sigma^{2}$ becomes linear in $t$, this asymptote and the rate of approach gives information about the structure of the medium. Free diffusion and restricted open diffusion are shown graphically in figures 2.3 and 2.4(a). Figure 2.5(b) shows the growth of $\sigma^{2}$ as a function of time for free diffusion (black) and restricted closed diffusion (red). At long times the growth of $\sigma^{2}$ becomes limited by the size of the pore and $D(t)$ becomes zero. This is shown graphically in figure 2.4(c). 


\section{Dispersion}

In the case of a flow field imposed by a pressure gradient the continuity equation becomes

$$
\frac{\partial C}{\partial t}+\nabla \cdot(\mathbf{v} C)+\nabla \cdot J=0
$$

where $V$ describes the velocity field. Again, $J$ is approximated by Fick's law of diffusion giving the convection-diffusion equation

$$
\frac{\partial C}{\partial t}+\nabla \cdot(\mathbf{v} C)-\nabla \cdot(D \cdot \nabla C)=0 .
$$

This equation describes the spreading of tracer particles and as such, there is general interest in the moments of the spreading. In an analogy to diffusion we look at the centralised mean-squared displacement

$$
\boldsymbol{\sigma}^{2}=\left\langle(\mathbf{r}(t)-\mathbf{r}(0))^{2}\right\rangle-\langle\mathbf{r}(t)-\mathbf{r}(0)\rangle^{2},
$$

this is the mean-squared displacement with respect to the bulk flow, henceforth the 'centralised' will be dropped and is referred to simply as 'meansquared displacement'.

Typically at long times, the growth of $\sigma^{2}$ is proportional to time so that a definition of the dispersion tensor can be made as $[46 ; 48]$

$$
\mathbf{D}^{*}=\lim _{t \rightarrow \infty} \frac{1}{2} \frac{\partial \boldsymbol{\sigma}^{2}}{\partial t}
$$

The definition of the Dispersion tensor includes the asymptote. In contrast to diffusive systems, dispersive systems typically have longer time scales such that in some systems the existence of asymptotic conditions is debated [49]. Hence methods for studying and quantifying the approach to asymptotic conditions have been developed [29], particulary using NMR. These methods are primarily applied to heterogeneous materials. Equation 2.14 allows for a time-dependent definition of dispersion as 


$$
\mathbf{D}^{*}(t)=\frac{1}{2} \frac{\partial \boldsymbol{\sigma}^{2}}{\partial t}
$$

typically in NMR studies a simpler definition of

$$
\mathbf{D}^{*}(t)=\frac{\boldsymbol{\sigma}^{2}}{2 t}
$$

is used. The implications of the two definitions will be discussed in section 5.7 .

\subsubsection{Propagators}

The Green's function for the diffusion equation also allows the definition of propagator [45], which is also a solution to the equation 2.8. NMR is well suited for measuring the average propagator (see section 3.3.2), this describes the probability that a particle moves a certain displacement $\mathbf{R}$ in a time $\tau$. For free diffusion we have

$$
\bar{P}(\mathbf{R}, \tau)=\frac{1}{(4 \pi D \tau)^{n / 2}} \exp \left(\frac{-\mathbf{R} \cdot \mathbf{R}}{4 D \tau}\right)
$$

where $n$ is the number of dimensions in which $\mathbf{R}$ is expressed. The convention of using capital letters to denote displacement and lower case letters to represent absolute position will be continued.

Using an eigenfunction expansion [45; 50], one can find the displacement propagator for closed geometries and model systems for an open restricted system [51].

In steady state, one can measure the distribution of displacements for a given encoding time. For very short times, the displacement will be dominated by diffusion. At intermediate times the displacement distribution will represent a histogram of the velocity field. Finally, at long times when particles have sampled the entire velocity field, the displacement propagator will tend to a Gaussian shape. Propagators for diffusion are shown in figure 2.3 and for dispersion in pipe flow in figure 2.6. 


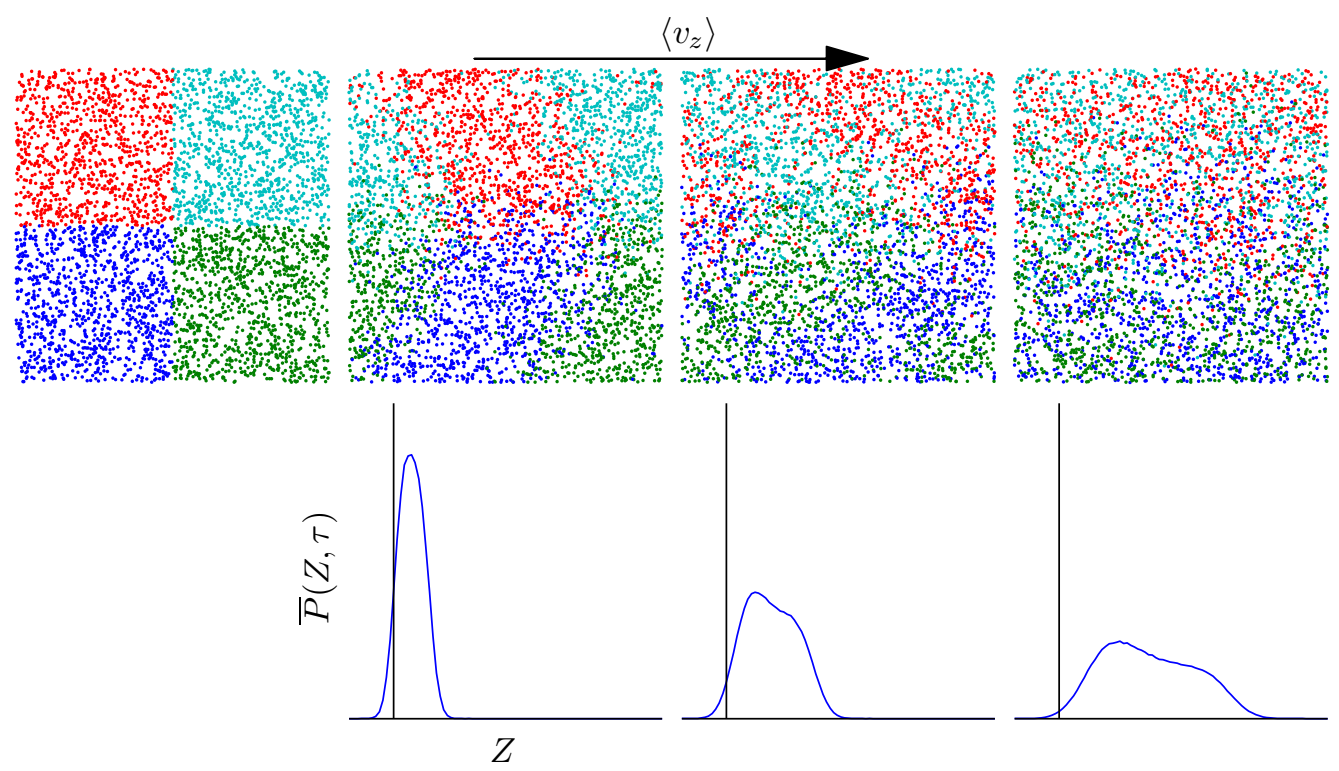

Figure 2.6: The dispersion of tracer particles in a parabolic flow shown at evolving times. The different colours are labels only. The boundaries are periodic in the $z$ direction and fully reflective transverse to the velocity. The average propagators, $\bar{P}(Z, \tau)$ describe the probability of particles moving a distance $Z$ in a time $\tau$. The effect of a bulk flow results in the average propagators being centered around the mean flow.

Propagators give an indication of the approach to asymptotic conditions, a measure of heterogeneity and provide a way of differentiating between the static and moving fractions of a system.

\subsubsection{The Peclet Number}

Dispersive systems can be characterised by the non-dimensional Peclet number, this describes the ratio of velocity gradient effects in relation to diffusion effects. It is given by

$$
P e=\frac{\langle v\rangle l}{D_{0}},
$$




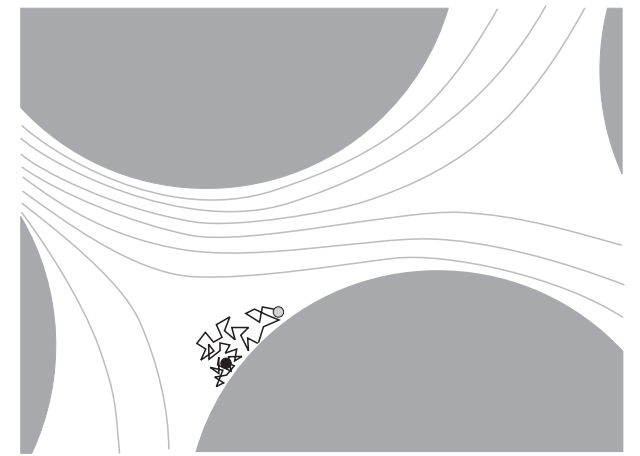

(a)
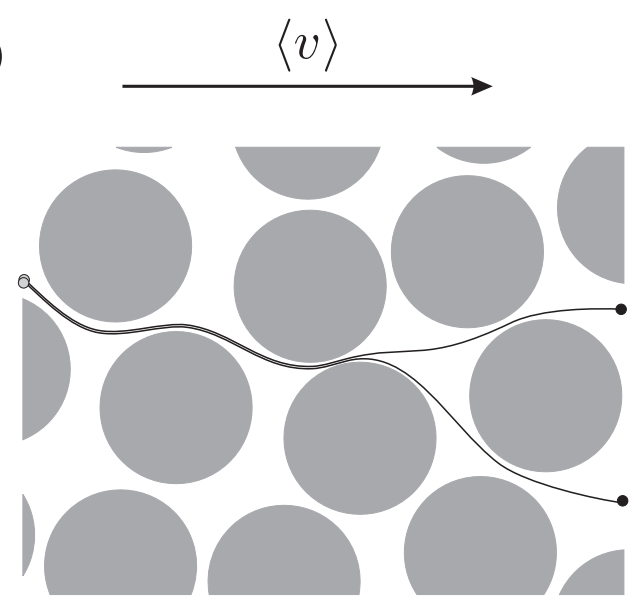

(c)

\section{$\langle v\rangle$}

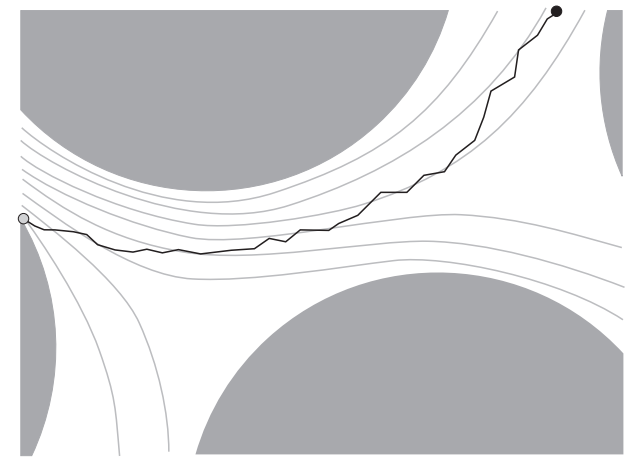

(b)

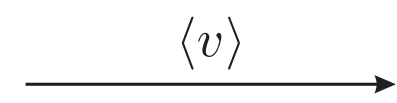

Figure 2.7: The three main mechanisms for dispersion in stationary velocity fields. Panel (a) shows 'hold-up' dispersion, a particle in a region of low velocity and mixing is dominated by diffusive effects. Panel (b) shows 'Taylor dispersion', where a tracer diffuses between stream lines. Panel (c) shows 'Mechanical dispersion', driven by the large length scale bifurcation of streamlines. 

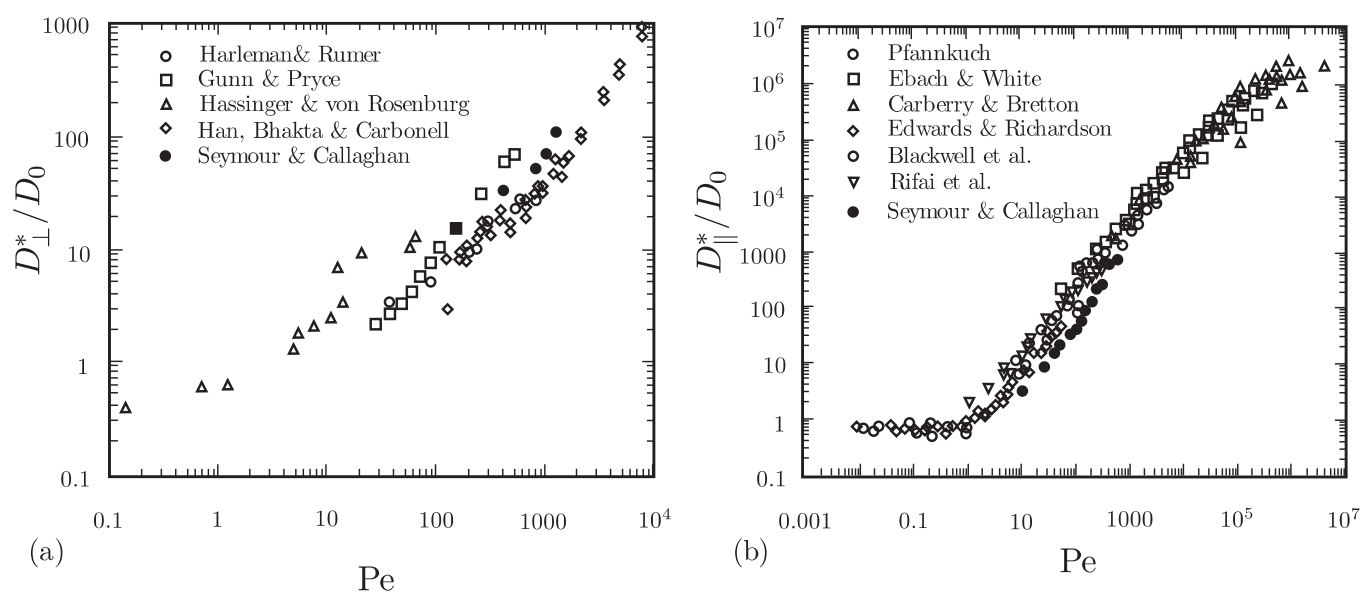

Figure 2.8: The universal curve for transverse (a) and longitudinal (b) dispersion. The NMR data labelled 'Seymour and Callaghan' [53] sit on the lower bound in the case of longitudinal dispersion.

where $l$ is the characteristic length. For flow in a random beadpack the Peclet number can be defined by [52]

$$
P e=\frac{\langle v\rangle d_{\text {bead }}}{D_{0}} \frac{\phi}{1-\phi}
$$

Longitudinal dispersion broadly follows a universal curve, shown in figure 2.8(b) The three main mechanisms of dispersion can be seen, holdup, diffusion dominated dispersion, (see figure 2.7(a)) at low Peclet number, where $D^{*} \approx D_{0}$, Taylor dispersion (see figure $2.7(\mathrm{~b})$ ) where $D^{*} \sim P e^{\beta}$ where $\beta \approx 1.2$ and mechanical dispersion at high Peclet numbers (see figure 2.7(c)), where $D^{*} \sim P e$ [2]. This curve has been compiled using a variety of different techniques, typically the NMR data sits on the lower bounds [14]. The transverse dispersion coefficients are a little more scattered (see figure 2.7(a)) and are, for high $P e$, several magnitudes lower than the longitudinal coefficients. The distinction between the three main mechanisms of dispersion is not seen as clearly. 


\subsubsection{Eulerian and Lagrangian}

There are two common descriptions of fluid dynamics, referred to as the Eulerian and the Lagrangian descriptions. In an Eulerian approach the fluid is described by considering the velocity at a fixed point in space as a function of time. This creates a velocity field that can be denoted by $\mathbf{v}_{E}(\mathbf{r}, t)$, or for stationary, time independent flow simply $\mathbf{v}_{E}(\mathbf{r})$. Associated with an Eulerian flow field is a probability density, $\rho(\mathbf{r}, t)$ which describes the probability of a fluid element being at position $\mathbf{r}$ and time $t$. Often the interest is in the velocity that fluctuates, in space, with respect to the mean, these velocity fields are denoted by $\mathbf{u}$. To describe the mean of the velocity field, an integration over all space is required, weighted by a probability density $\rho(\mathbf{r})$, giving

$$
\overline{\mathbf{v}}=\int \rho(\mathbf{r}) \mathbf{v}_{E}(\mathbf{r}) d \mathbf{r}
$$

The fluctuating part of a stationary velocity field can then be written as $\mathbf{u}_{E}(\mathbf{r})=\mathbf{v}_{E}(\mathbf{r})-\overline{\mathbf{v}}$.

A Lagrangian approach is one where the coordinates of an individual particle or volume element is tracked over time [2] This creates a set of elements given by $\mathbf{v}^{i}(\mathbf{r}(t))$. There is a distinction in the time dependence in the two formalisms since a Lagrangian description still includes a time dependance for a stationary flow. For a stationary flow field we typically write this as $\mathbf{v}^{i}(\mathbf{r}(\tau))$ and sometimes simply as $\mathbf{v}^{i}(\tau)$.

The average velocity of a Lagrangian velocity description is the numerical mean of the set of tracers. For a stationary velocity field the mean will be independent of time

$$
\overline{\mathbf{v}}=\frac{1}{N} \sum_{i=1}^{N} \mathbf{v}_{L}^{i}
$$

where the superscript $i$, refers to the particle or volume element label.

An Eulerian description of stationary flow field, will not contain the information about particles moving across stream lines, and the effect of 
diffusive mixing. Such information is described in the propagator. The propagator and the probability distribution function provide the link between the Eulerian and Lagrangian formalisms.

In the experimental limit of instantaneous velocity encoding (see section 3.3.3), NMR velocimetry can provide a complete, three dimensional, Eulerian flow field. A NMR image provides the probability density. Other NMR experiments such as PGSE experiments are more suited to a Lagrangian description. The ensemble average notation $\langle\ldots\rangle$ typically used in PGSE NMR can refer to averages in both formalisms.

\subsubsection{The Velocity Autocorrelation Function}

A Lagrangian description of a flow field is a natural way for the definition of a velocity autocorrelation function (VACF). This provides some temporal structure to the mixing. The definition is more natural using the the Lagrangian description. In a stationary velocity field, a time-dependent quantity can be defined that describes the rate that a particle loses memory of its initial velocity

$$
\langle\mathbf{u}(0) \mathbf{u}(\tau)\rangle=\frac{1}{N} \sum_{i=1}^{N} \mathbf{u}_{L}^{i}(0) \cdot \mathbf{u}_{L}^{i}(\tau),
$$

where the fluctuating velocity $\mathbf{u}(\tau)$ is with respect to the mean, that is $\mathbf{u}(\tau)=\mathbf{v}(\tau)-\overline{\mathbf{v}}$.

The velocity autocorrelation function also provides an equivalent description of dispersion. The displacement of a single tracer can be expressed as the time integral of its velocity

$$
\mathbf{r}(\tau)-\mathbf{r}(0)=\int_{0}^{\tau} \mathbf{v}(t) d t
$$

alternatively, integration over the $\mathbf{u}$ velocity will give the displacement with respect to the mean. Taking the ensemble average means we can express the centralised mean-squared displacement as 


$$
\boldsymbol{\sigma}^{2}(\tau)=\left\langle\left(\int_{0}^{\tau} \mathbf{u}(t) d t\right)\left(\int_{0}^{\tau} \mathbf{u}\left(t^{\prime}\right) d t^{\prime}\right)\right\rangle
$$

The independent integrals can then be combined and the stationary velocity field means the integral of the ensemble average is the same as the ensemble average of the integral giving

$$
\boldsymbol{\sigma}^{2}(\tau)=\int_{0}^{\tau} \int_{0}^{\tau}\left\langle\mathbf{u}(t) \mathbf{u}\left(t^{\prime}\right)\right\rangle d t d t^{\prime}
$$

The integrand here is a velocity autocorrelation function expressed in two time dimensions. The VACF is independent of the initial time such that $\langle\mathbf{u}(0) \mathbf{u}(0+\tau)\rangle=\langle\mathbf{u}(t) \mathbf{u}(t+\tau)\rangle$ and $\langle\mathbf{u}(0) \mathbf{u}(\tau)\rangle=\langle\mathbf{u}(\tau) \mathbf{u}(0)\rangle$. This enables the mean squared displacement to be written in terms of the VACF described by one time variable,

$$
\boldsymbol{\sigma}^{2}(\tau)=2 \int_{0}^{\tau} \int_{0}^{t^{\prime}}\langle\mathbf{u}(0) \mathbf{u}(t)\rangle d t d t^{\prime} .
$$

Note a change in variables giving the factor 2 and the integration limit to $t^{\prime}$. This allows the expression for the time dependent dispersion in equation 2.15 to be expressed as

$$
\mathbf{D}^{*}(\tau)=\int_{0}^{\tau}\langle\mathbf{u}(0) \mathbf{u}(t)\rangle d t
$$

This expression relies on an underlying velocity field and so has no meaning for pure diffusion. In fact the pure diffusive part of the dispersion is not included in this definition. This has led to the often quoted asymptotic dispersion for pipe flow as $D^{*}=\frac{\bar{v}^{2} a^{2}}{48 D_{0}}$ [54], when it should be $D^{*}=$ $\frac{\bar{v}^{2} a^{2}}{48 D_{0}}+D_{0}$ [55]. For dispersion in porous media this will be a small effect for large Peclet numbers $(>100)$. For smaller Peclet numbers the discrepancy will be a measure of the time dependent diffusion and hence the tortuosity. 


\subsection{Nonlocal Dispersion}

\subsubsection{Non-Fickian Dispersion}

The conventional advection-diffusion equation 2.11, has been shown to be unable to fully predict dispersive effects, even for dispersion in pipe flow [56]. Anomalous, or non-Fickian, dispersion has been observed in a large number of systems in the field [57-60], laboratory [61-65] and specifically with PGSE NMR [66; 67]. In general, these effects are observed in heterogenous materials such that the assumption of purely deterministic behaviour over a representative elementary volume (REV) is not sufficient [68]. The need for an improved description of dispersion is well summerised by Sternburg et al.[65] "These observations suggest that dispersion in scale dependent media is non-Fickian and that another constitutive theory must be identified. We believe that this theory must have nonlocal character".

\subsubsection{Nonlocality}

In physics, a nonlocal theory is one in which the description of the system involves some interaction of particles or other parts of the system separated by space and or time. Nonlocality has been treated extensively in classical electrodynamics where the most general connection between the electric displacement field and the electric field in a dielectric is nonlocal [69]. Nonlocal equations describe the behaviour at a point by integrating over the entire system and integration over its history. The equations that describe such a behaviour are characterised by containing integrals and derivatives $[68 ; 70]$.

Several such theories exist for describing non-Fickian dispersion in porous media. In 1995 Cushman and $\mathrm{Hu}$ [71] published "A resumé of nonlocal transport theories". More recently Neuman and Tartakovsky [68] published a review discussing theories of non-Fickian transport in hetero- 
geneous media. One such nonlocal theory from Koch and Brady [13] is described as 'Space-time convolution-Fickian dispersion' [71] and 'spacetime nonlocal' [68]. Associated with each nonlocal theory is at least one nonlocal dispersion tensor. It is the nonlocal dispersion tensor, denoted here by $\mathbf{D}^{N L}$, from the theory of Koch and Brady with which we will be concerned.

A nonlocal dispersion theory is one in which the behaviour at any point can not simply be described by the local spatial environment at a given time. Instead, to be able to describe the behaviour at a particular point, one much have knowledge of the system over all space and its complete history. The nonlocal dispersion tensor therefore describes the correlations between particles separated by space and time.

\subsubsection{Koch and Brady}

The nonlocal theory of Koch and Brady, and specifically the nonlocal dispersion tensor, is a quantity recognised as being of fundamental importance in the NMR characterisation of fluid dispersion [14-24], particularly at short times and at length scales comparable to a pore.

Koch and Brady proposed a nonlocal description of dispersion where the tracer flux is given by $[13 ; 71]$

$$
\mathbf{q}=\langle\mathbf{v}(t)\rangle C\left(\mathbf{R}^{\prime}, t\right)-\int_{0}^{t} \int \mathbf{D}^{N L}(\mathbf{R}, \tau) \cdot \nabla C\left(\mathbf{R}^{\prime}-\mathbf{R}, t-\tau\right) d \mathbf{R} d \tau .
$$

Here we have followed [72] by neglecting the small terms associated with spatial changes in the average concentration and fluctuation of concentration caused by any sources. The nonlocal dispersion tensor $\mathbf{D}^{N L}$ is defined as

$$
\mathbf{D}^{N L}(\mathbf{R}, \tau)=\int \mathbf{u}_{E}(\mathbf{r}, 0) P(\mathbf{r}) P(\mathbf{r} \mid \mathbf{r}+\mathbf{R}, \tau) \mathbf{u}_{E}(\mathbf{r}+\mathbf{R}, \tau) d \mathbf{r}
$$


This definition is of a quantity that is well defined at all times and which has the potential to describe dispersive effects and short displacements. The nonlocal dispersion tensor has the potential to be used for calculations in nonlocal theories, and therefore to model concentration profile changes and residence time distributions that Fick's law is unable to model. As will be shown in chapters 7 to 9 , components of the nonlocal dispersion tensor itself are useful for inferring properties of the flow and describing correlations.

The relationship between the nonlocal dispersion tensor $\mathbf{D}^{N L}$ and the conventional dispersion tensor $\mathrm{D}^{*}$ can be seen from consideration of the velocity autocorrelation function. The definition of the dispersion tensor, equation 2.15, can also be represented through the time integral of the velocity autocorrelation function. The dispersion tensor is asymptotic in time and contains no spatial, or displacement, information. A nonlocal description requires some displacement information. This can be found by considering the VACF, which is naturally expressed as an ensemble average of the Lagrangian velocities, with an Eulerian description.

Using the notation of a steady state Eulerian velocity field from section 2.3.4 we can rewrite the expression for the VACF using Eulerian velocities. The velocity autocorrelation function as calculated from a set of Lagrangian particles is

$$
\langle\mathbf{u}(0) \mathbf{u}(\tau)\rangle=\frac{1}{N} \sum_{i=1}^{N} \mathbf{u}_{L}^{i}(0) \cdot \mathbf{u}_{L}^{i}(\tau) .
$$

Here we have some temporal information about the decay of correlation, but there is no spatial information. In order to have a more complete description of dispersion and correlations it is desirable to have some sort of spatial, or in this case displacement resolution. The nonlocal dispersion tensor provides these details and the definition of $\mathbf{D}^{N L}$ can be seen by rewriting the expression for the velocity autocorrelation function using Eulerian velocities. The stationary field of Eulerian velocities contains no 
information about how particles move from one region to another, this information is provided by the propagator $P\left(\mathbf{r}, 0, \mathbf{r}^{\prime}, \tau\right)$. This describes the probability that a particle at position $r$ at time 0 , will be found at position $\mathbf{r}^{\prime}$ at time $\tau$. Thus the VACF can be written as a correlation integral,

$$
\left\langle\mathbf{u}_{L}(0) \mathbf{u}_{L}(\tau)\right\rangle=\iint \mathbf{u}_{E}(\mathbf{r}, 0) P(\mathbf{r}) P\left(\mathbf{r} \mid \mathbf{r}^{\prime}, \tau\right) \mathbf{u}_{E}\left(\mathbf{r}^{\prime}\right) d \mathbf{r}^{\prime} d \mathbf{r}
$$

which contains spatial correlation information buried in the VACF. Rewriting equation 2.31 in terms of relative displacements $\mathbf{R}=\mathbf{r}^{\prime}-\mathbf{r}$ and initial position we have

$$
\left\langle\mathbf{u}_{L}(0) \mathbf{u}_{L}(\tau)\right\rangle=\iint \mathbf{u}_{E}(\mathbf{r}) P(\mathbf{r}, 0) P(\mathbf{r} \mid \mathbf{r}+\mathbf{R}, \tau) \mathbf{u}_{E}(\mathbf{r}+\mathbf{R}) d \mathbf{r} d \mathbf{R}
$$

which enables the integrand of the integral over displacement $\mathbf{R}$, to be seen as the nonlocal dispersion tensor,

$$
\mathbf{D}^{N L}(\mathbf{R}, \tau)=\int \mathbf{u}_{E}(\mathbf{r}) P(\mathbf{r}) P(\mathbf{r} \mid \mathbf{r}+\mathbf{R}, \tau) \mathbf{u}_{E}(\mathbf{r}+\mathbf{R}) d \mathbf{r}
$$

This can be written, using the compact $\langle\ldots\rangle$ notation, as

$$
\mathbf{D}^{N L}(\mathbf{R}, \tau)=\left\langle\mathbf{u}_{E}(0) \bar{P}(\mathbf{R}, \tau) \mathbf{u}_{E}(\tau)\right\rangle
$$

The compact notation of the expression in equation 2.34 will be used interchangeably with the explicit definition is given in equation 2.29. The subscripts denoting Eulerian velocities will be omitted. Although this derivation has used a stationary velocity field such that $\mathbf{u}(\mathbf{r}, 0)=\mathbf{u}(\mathbf{r}, t)$, the expression on the right hand side of equation 2.34 has an apparent time dependence in the velocities. This time variable describes a temporal correlation. The spatial and displacement information of the velocities is implied through the average propagator. The nonlocal dispersion tensor thus describes a displacement resolved, velocity autocorrelation function. The extra information in this quantity will be highlighted in further chapters. 
It is clear that the conventional dispersion tensor can be recovered via

$$
\mathbf{D}^{*}=\lim _{t \rightarrow \infty} \int_{0}^{t} d \tau \int \mathbf{D}^{N L}(\mathbf{R}, \tau) d \mathbf{R} .
$$

The nonlocal dispersion tensor therefore contains all the temporal and displacement information that is averaged out in the dispersion tensor.

\subsubsection{Measurement of $\mathrm{D}^{N L}$}

As will be seen in chapters 6 to 9, components of the nonlocal dispersion tensor can be measured using NMR, and simulated using a lattice Boltzmann based simulation suite. Typically these components are plotted as functions of continuous displacement. The nonlocal dispersion tensor is also continuous in time, measurements over a wide range of times are somewhat tedious for NMR but are suited for simulations.

Typically, in experiments, the displacement is resolved in only one Cartesian dimension. This gives a term which is an average over two other displacement directions. In the example of encoding for $Z$ displacement we would have

$$
\mathbf{D}^{N L}(Z, \tau)=\int \mathbf{u}_{E}(\mathbf{r}) P(\mathbf{r}) P(\mathbf{r} \mid \mathbf{r}+Z, \tau) \mathbf{u}_{E}(\mathbf{r}+Z) d \mathbf{r}
$$

which can also be written as

$$
\mathbf{D}^{N L}(Z, \tau)=\iint \mathbf{D}^{N L}(\mathbf{R}, \tau) d X d Y
$$

A choice of the direction for the velocity encoding, such as $z$, would then give us a term $D_{z z}^{N L}(Z, \tau)$ where the two subscripts relate to the direction chosen for the final and initial velocity encoding. These terms are referred to as 'one-dimensional components'.

An encoding that contains all three displacement directions would be highly desirable, and indeed, essential for use in using equation 2.28 for modelling flux concentration. However, as discussed in section 8.1 such 
an experimental encoding is technically difficult. Nevertheless in chapter 8 these higher dimensional encodings have been investigated in simulations. Insights into the behaviour of particles at the pore scale can be readily inferred from the measurement of one-dimensional components. It is here where the focus of this thesis lies.

\subsection{Conclusions}

The basic language of dispersion in porous media has been introduced and an introduction to non-Fickian effects provided. A number of theories have been put forward to model such behaviour, and the theory from Koch and Brady [13] contains a tensor that we show is measurable with NMR. It is not the intention of this thesis to verify this particular nonlocal theory, but to develop the methodology to measure the components of the tensor with NMR and to then use these components to infer properties of the dispersion.

Pulsed Gradient Spin Echo (PGSE) NMR techniques, as discussed in chapter 3, are ideal for measuring ensemble properties of flow in opaque systems such as porous media. One difficulty is the limited time scale accessible making asymptotic conditions difficult to determine. However, considerable information can be obtained at shorter time scales. The nonlocal dispersion tensor of Koch and Brady has been adopted by the NMR community as the ideal way to describe correlation effects at a pore size scale. The measurement of the nonlocal tensor components provides a well defined way of quantifying dispersive and correlation effects at short times and distances. 


\section{Chapter 3}

\section{Nuclear Magnetic Resonance}

\subsection{Introduction}

The two most widespread uses of magnetic resonance are imaging, commonly called MRI (magnetic resonance imaging) and spectroscopy. MRI has become routine and indispensable in clinical diagnostics. The noninvasive nature and the relatively high spatial resolution of measurements result in convenient diagnoses. Further information through the use of contrast agents increases the ability to detect cancerous tumours. Recently, functional MRI techniques have been developed to enable spatial localisation of brain activity. Nuclear Magnetic Resonance Spectroscopy is a measurement technique whereby the signal from a nucleus is very sensitive to its local, molecular environment. This permits identification of chemical species, structure determination of large molecules such as proteins and local orientation.

In this thesis we will be concerned primarily with measuring particle displacements, using Pulsed Gradient Spin Echo (PGSE) techniques. These techniques will be covered in some detail in this chapter and chapter 5 . Full descriptions of imaging and spectroscopy techniques can be found in $[73 ; 74]$ and $[75 ; 76]$, respectively. 


\subsection{The Semi-Classical Model}

Many of the simple phenomena in Nuclear Magnetic Resonance, particulary when studying imaging and diffusion, can be explained by what is known as The semi-classical model. This is where a sample, on being placed in a magnetic field, acquires a bulk magnetisation. The evolution of the bulk magnetisation can be understood through classical laws of torque and free-precession. Such an approach will suffice for describing the phenomena contained in this thesis. A simple quantum mechanical background of nuclei in a magnetic field is given to explain the origin of the bulk magnetisation. For a full quantum mechanical treatment, essential for many facets of NMR, the reader is referred to standard texts such as [77].

\subsubsection{Quantum Mechanical Background}

Certain nuclei possess a net quantum mechanical angular momentum or spin. This is a vector quantity and collinear to this is a magnetic dipole moment. The net spin of a nucleus is described by the quantum number $I$, which can take integer or half integer values. Examples of spin $1 / 2$ nuclei commonly studied using NMR are ${ }^{1} \mathrm{H},{ }^{13} \mathrm{C}$ and ${ }^{19} \mathrm{~F}$. Other nuclei studied using NMR include ${ }^{2} \mathrm{H}$ with spin 1 and ${ }^{23} \mathrm{Na}$ with spin $3 / 2$. The total angular momentum, $\mathbf{I}$, for a nucleus has a magnitude of $\hbar \sqrt{I(I+1)}$. In this thesis we will look solely at the ${ }^{1} \mathrm{H}$ nucleus.

Under a zero magnetic field nuclei are free to choose any orientation as shown in figure 3.1(a). Imposing a magnetic field $\mathbf{B}$ on the nuclei results in a torque, and therefore some alignment (as shown in figure 3.1(b)), due to the magnetic moment. The quantisation of energy restricts the nuclei to $2 I+1$ allowable orientations labelled by $m$, where $m$ varies from $-I$ to $I$. The orientations are projections onto a quantisation axis (in this case the direction of the magnetic field). Note that the orientations of the moments are not collinear with the magnetic field but for the purposes of this 


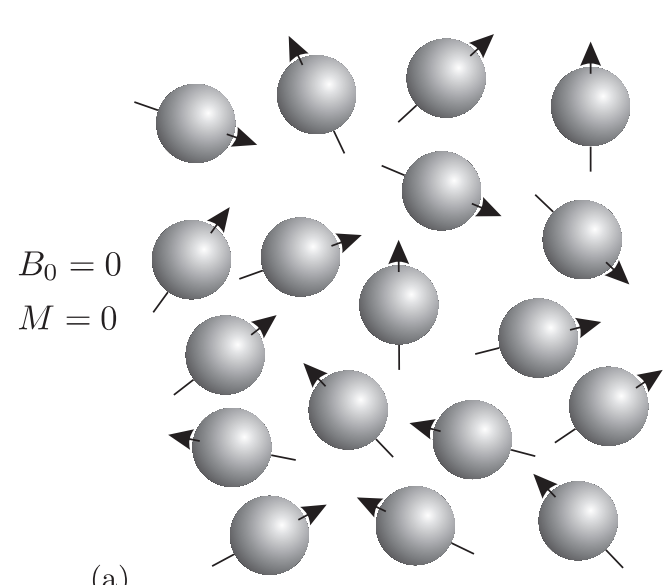

(a)

Figure 3.1: In a zero magnetic field, spins are free to choose any orientation, as shown in panel (a) Imposing a magnetic field $B_{0}$ on a set of nuclear spins will cause alignment as shown in panel (b). For the purposes of this discussion the spins can be treated as either parallel or anti-parallel to the main field [78]. The population given from the Boltzmann distribution will give the set of nuclei with a net magnetisation $M$.

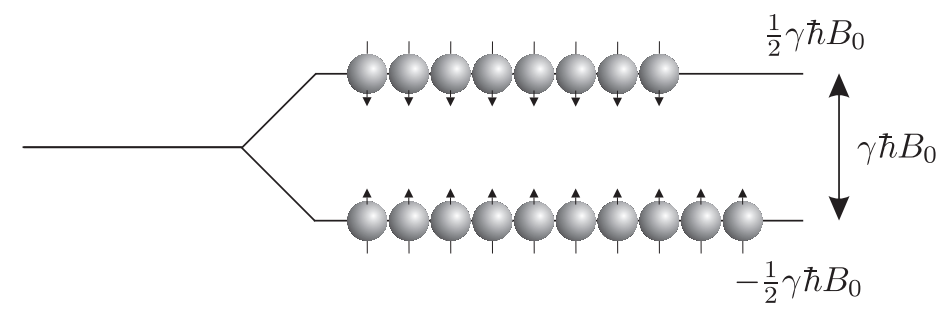

Figure 3.2: The energy levels due to the Zeeman interaction for a spin $1 / 2 \mathrm{nu}-$ cleus. The population difference between the two states is exaggerated in this figure. 
discussion they can be treated as parallel or antiparallel [78]. The energy level for each orientation is given by

$$
E_{m}=-\gamma \hbar m B_{0}
$$

where $\gamma$ is the magnetogyric ratio of the nuclei and $\hbar$ is Planck's constant divided by $2 \pi$. The splitting into $2 I+1$ energy levels is known as the Zeeman interaction, and is shown for a spin $1 / 2$ nucleus in figure 3.2. The difference in the two energy levels for a spin $1 / 2$ system is given by

$$
\Delta E=\gamma \hbar B_{0}
$$

The population of states, $P_{m}$ is given by the Boltzmann distribution. For a spin $1 / 2$ nucleus at room temperature and laboratory magnetic fields the difference in population can be approximated very well as

$$
P_{-\frac{1}{2}}-P_{\frac{1}{2}}=\frac{\gamma \hbar B_{0}}{2 k_{B} T}
$$

where $k_{B}$ is Boltzmann's constant and $T$ is the temperature. Nuclei can be excited from a lower energy state to that of a higher energy by absorbing a photon with the correct polarisation and an energy equal to that of equation 3.2 that is, with a frequency $\omega=\gamma B_{0}$. On relaxing back to equilibrium, a photon is emitted.

\subsubsection{Bulk Magnetisation}

For the purposes of describing the experiments henceforth the magnetisation from the nuclei can be summed up to give a bulk magnetisation, $\mathbf{M}_{0}$. The angular momentum is collinear with the bulk magnetisation. The bulk magnetisation of a sample can be given by

$$
M_{0}=N\left(P_{-\frac{1}{2}}-P_{\frac{1}{2}}\right) \mu_{z}=\frac{N \gamma^{2} \hbar^{2} B_{0}}{4 k_{B} T}
$$


where $N$ is the total number of nuclei in the sample. We have used the $z$ component of the magnetic moment as $\mu_{z}= \pm \frac{1}{2} \gamma \hbar$. The bulk magnetisation is aligned with the magnetic field, which is typically taken as being in the $z$-axis as shown in figure 3.3. Although not observable in this equilibrium state, the bulk magnetisation $M_{0}$ will precess around the $B_{0}$ field due to its angular momentum and the torque. The rate of precession is given by the Lamor frequency $\omega=\gamma B_{0}$. In order to detect this magnetisation it must be tipped into the transverse plane where the precession can be detected by the use of an induction coil. In the section 3.2.1 we saw that the nuclei can be excited into a higher state by absorbing a photon. In this semi-classical treatment we consider the excitation as the bulk magnetisation precessing into the transverse plane around a second orthogonal magnetic field, $B_{1}$.

\subsubsection{The Rotating Frame}

In order for a field $B_{1}$ to interact with the bulk magnetisation, it must be rotating with the same precession frequency. This is best visualised in the rotating frame. The bulk magnetisation and the $B_{0}$ field are shown in the laboratory frame, $x_{l a b}, y_{l a b}$ and $z$ in figure 3.3(a). If a new rotating frame, $x_{r o t}, y_{\text {rot }}$ and $z$ is defined as rotating with the precession frequency, the precession of the bulk magnetisation is no longer observed and hence the effective $B_{0}$ is now zero (figure 3.3(b)). The introduction of a field $B_{1}$ which is static along $x_{\text {rot }}$ in the rotating frame (and hence rotating in the laboratory frame) causes a new precession of the bulk magnetisation (figure 3.3(c)). For a given strength of a $B_{1}$ field it is possible to apply the field for a time such that the bulk magnetisation has rotated into the $x_{\text {rot }}-$ $y_{\text {rot }}$ plane. As the rotation has moved through $\pi / 2$ radians the application of the $B_{1}$ field is known as a ' $\pi / 2$ pulse'. A $B_{1}$ field that is applied for a time such that the magnetisation has rotated through $\pi$ radians is called a ' $\pi$ pulse' 


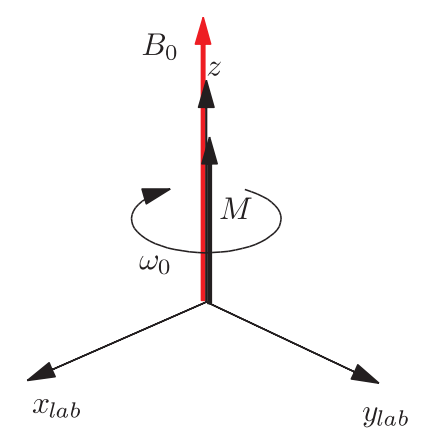

(a)

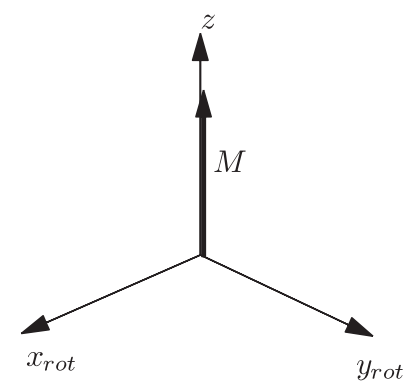

(b)

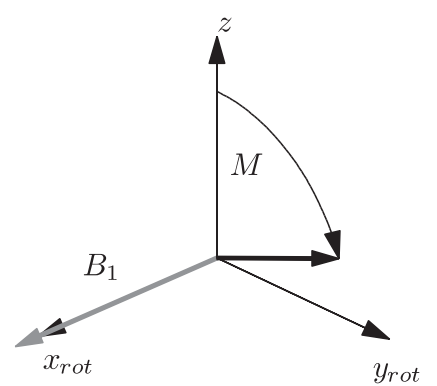

(c)

Figure 3.3: Panel (a) shows the magnetic field $B_{0}$ and the precessing bulk magnetisation $M$ (Not to scale). If a rotating coordinate frame is used, $M$ is stationary and the field $B_{0}$ has no effect (panel (b)). Shown in panel(c) is a second field $B_{1}$ which causes the magnetisation to precess into the transverse plane.

A rotating $B_{1}$ field can be generated by considering a sinusoidal pulse $2 B_{1} \sin \omega t$. This can be considered as a superpostition of two pulses such that $2 B_{1} \sin \omega t=B_{1} \exp (i \omega t)-B_{1} \exp (-i \omega t)$. Where the complex plane represents the $x_{l a b}-y_{l a b}$ plane. The leaves a static field of magnitude $B_{1}$ in the rotating frame. In the counter-rotating frame where the second component of $2 B_{1} \sin \omega t$ is stationary, the $B_{0}$ field is completely dominant.

The semi-classical approach and the rotating frame provides a convenient framework for further discussions involving manipulation of the magnetisation. Henceforth, in later sections, the rotating frame will be used exclusively. The rotating frame will be referred to simply as $x, y$ and $z$. Further discussions of the evolution and manipulation will treat the sample as a distribution of bulk magnetisations, each experiencing its own local magnetic field.

\subsubsection{The Bloch Equations}

The tipping of the bulk magnetisation into the transverse plane as shown in figure 3.3(c) puts the system into a state away from thermal equilibrium 
and in some cases a measurable temperature change is observed [79]. The time evolution of the magnetisation is given by the Bloch equation, considering $\mathbf{M} / \gamma$ as angular momentum,

$$
\frac{d \mathbf{M}}{d t}=\gamma \mathbf{M}(t) \times \mathbf{B}(t)-\mathbf{R}(\mathbf{M}(t)-\mathbf{M}(0)),
$$

where the relaxation matrix $\mathbf{R}$ has the elements $R_{11}=R_{22}=T_{2}$ and $R_{33}=T_{1}$. These times are referred to as the transverse relaxation and the longitudinal relaxation respectively.

Following the $\pi / 2$ pulse in figure 3.3(c) where the magnetisation is now wholly in the $x$-axis (of the rotating frame), the component of the magnetisation in the transverse plane will relax with a timescale, $T_{2}$. The relaxation can be described by

$$
\frac{\mathrm{d} M_{x}}{\mathrm{~d} t}=-\frac{M_{x}}{T_{2}}
$$

so that as a function of time we have

$$
M_{x}=M_{x} \exp \left(-t / T_{2}\right)
$$

The relaxation back to thermal equilibrium is described phenologically by

$$
\frac{\mathrm{d} M_{z}}{\mathrm{~d} t}=\frac{M_{0}-M_{z}}{T_{1}}
$$

where $T_{1}$ is also known as the spin-lattice relaxation or equivalently the longitudinal relaxation. This relaxation represents the loss of energy to the nuclei surroundings.

The solution to this gives

$$
M_{z}(t)=M_{z}(0) \exp \left(-t / T_{1}\right)+M_{0}\left(1-\exp \left(-t / T_{1}\right)\right) .
$$

A description of the pulse sequences used to measure $T_{1}$ and $T_{2}$ is given in section 3.2.6. 


\subsubsection{Excitation and Detection}

For this thesis we will be looking solely at ${ }^{1} \mathrm{H}$ nuclei, and in particular, water molecules. The magnetogyric ratio of ${ }^{1} \mathrm{H}$ nuclei (or known simply as protons) is $2.675 \times 10^{8}\left(\mathrm{rad} \mathrm{T}^{-1}\right)$. The liquid cryogen cooled superconducting magnet used for the experiments here provides a magnetic field of $9.40 \mathrm{~T}$ that gives a resonant frequency for protons at $400 \mathrm{MHz}$. Therefore the $B_{1}$ pulse is at the same frequency, referred to as a radio-frequency (RF) pulse.

To generate a $B_{1}$ field that is orthogonal to the main $B_{0}$ field a 'birdcage' resonant coil is used [80]. This is shown schematically in figure 3.4. The combination of inductive strips and capacitors provides the phase shifts necessary for a $\sin \theta$ current distribution, thus providing a reasonably homogeneous $B_{1}$ field. A pair of variable capacitors is provided in order to 'tune' the resonance of the coil to the correct frequency and to ensure the coil is impedance matched to $50 \Omega$.

The spins are detected in the transverse plane via an induced voltage in the RF coil. Heterodyne mixing is used in the detection to recover the magnitude and the phase shift of the acquired signal. The two components are referred to as the 'real' and 'imaginary' parts of the signal. The absolute phase of the acquired signal is arbitrary. Processing the signal in this method enables the extension of the rotating frame visualisation, so that phase shifts and frequency changes will appear centered around zero. Further details of the hardware and signal processing specific to NMR can be found in reference [78].

A pulse program describing a simple $\pi / 2$ excitation pulse is shown in figure 3.5. Once the spins are tipped into the transverse plane they will precess at slightly different frequencies due to inhomogeneities in the magnetic field. The summation of the phase changes of the spins will lead to a time dependent decay know as a free induction decay (FID). If the magnetic field is sufficiently homogeneous the structure of the FID will be due to the molecular environment of the nuclei, thus providing the basis of 


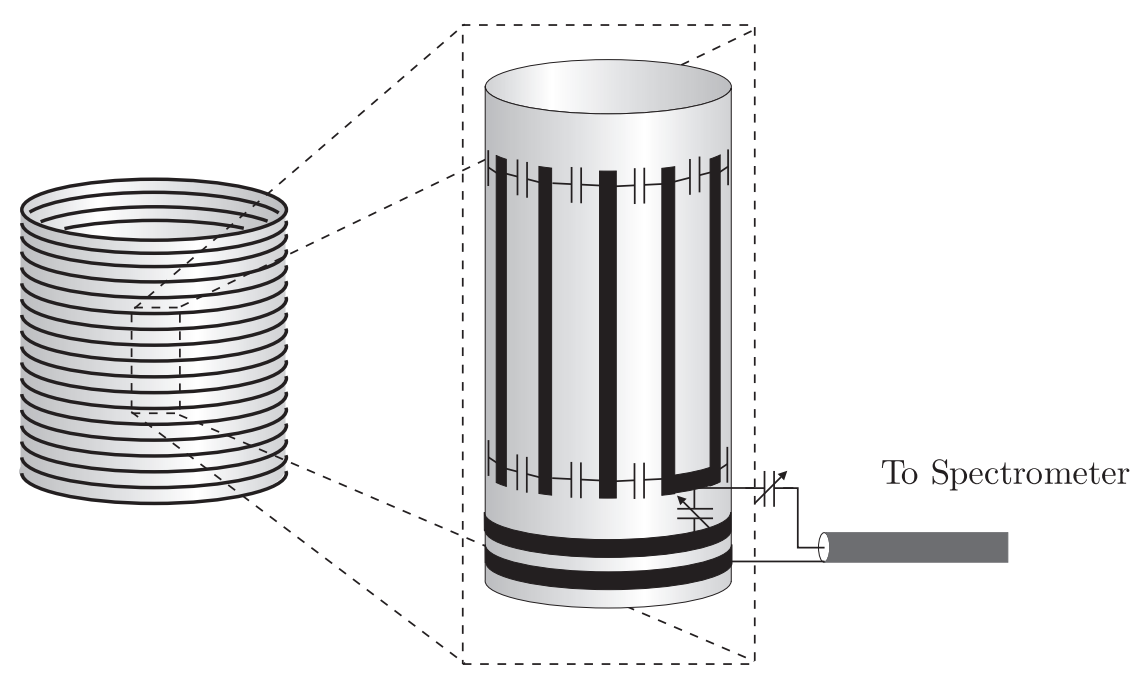

Figure 3.4: The static $B_{0}$ field is provided by a cryogen-cooled superconducting solenoid-like electromagnet. The 'birdcage' excitation and detection coil resonates and is impedance matched at the nuclear precession frequency. The phase shifts in the rings provide a sinusoidal current distribution giving a homogeneous $B_{1}$ 


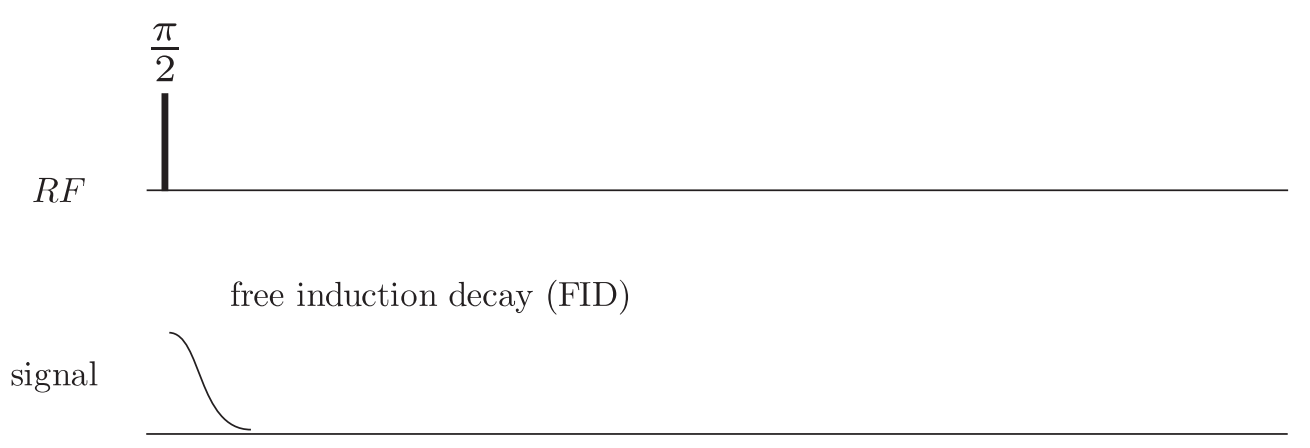

Figure 3.5: $\mathrm{A} \pi / 2$ excitation pulse will tip the spins out of equilibrium. A voltage will be induced in the excitaion/detection coil. The signal will decay due to the inhomogeneity of the magnetic field and therefore slightly different precession rates in the sample. The observed signal is called a free induction decay.

spectroscopy.

The signal loss due to magnetic inhomogeneities can be recovered by means of an NMR echo. Shown in figure 3.6 is a $\pi / 2$ excitation pulse followed by a free induction decay. At some time $t_{e}$ a pulse is applied with twice the length of the initial pulse. This second pulse has the effect of rotating the spins through $\pi$ radians into the transverse plane. This can be seen in figure 3.7. The spins then accumulate phase at the same rate as was previously lost, or vice versa, and then come into phase again forming a Hahn echo [81]. Note in figure 3.7(a) the initial $\pi / 2$ is oriented along the $x$ axis and the refocussing $\pi$ pulse is directed along the $y$ axis. This orientation can be determined by choosing the phase of the RF pulse. The time between the initial excitation pulse and the echo enables encoding for various measurements. The overwhelming bulk of NMR pulse sequences make use of an NMR echo.

\subsubsection{Relaxation}

The loss of signal after an NMR echo is further recoverable by subsequent $\pi$ pulses, this forms an echo train as shown in figure 3.8. After an initial 


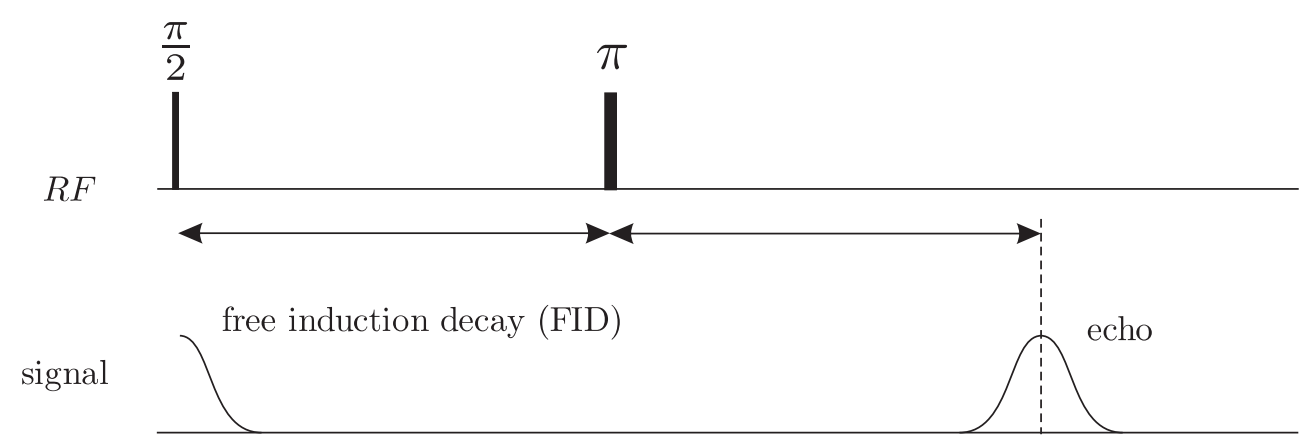

Figure 3.6: The dephasing seen following a $\pi / 2$ pulse can be refocussed into an 'echo'. The $\pi$ pulse has the effect of reversing the dephasing such that the spins will, at the echo time, superpose into a measurable signal. This is also shown in figure 3.7.

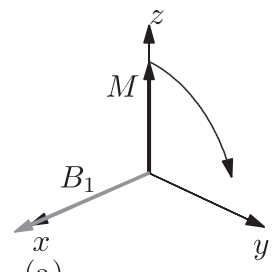

(a)

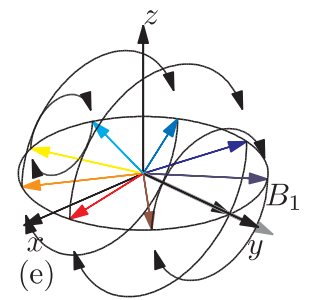

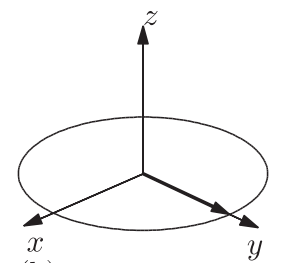

(b)

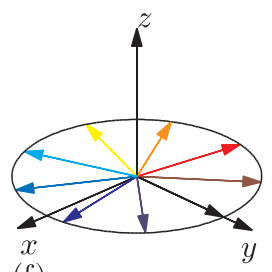

(f)

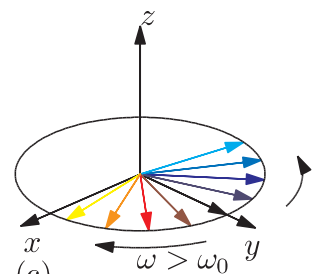

(c)

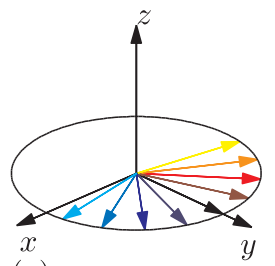

(g)

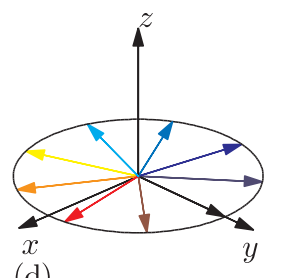

(d)

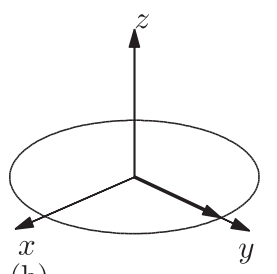

(h)

Figure 3.7: An excitation pulse along the $x$ axis (a) will tip the magnetisation into the $y$ axis (b). This pulse can be called $(\pi / 2)_{x}$. The spins will begin to dephase as shown in (c) and (d). The $\pi_{y}$ pulse in (e) will flip the phase of the spins so that the faster moving spins (red-yellow) and the slower moving spins (blue to cyan) will recover their phase (f) and (g) to form a Hahn echo in the $y$ axis (h). 


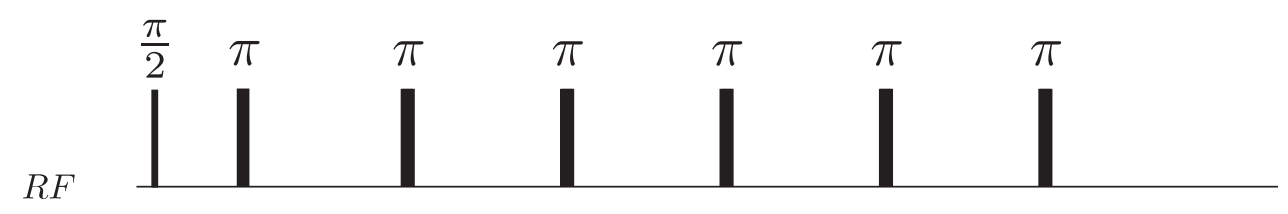

$$
E=E_{0} e^{-\frac{t}{T_{2}}}
$$

signal

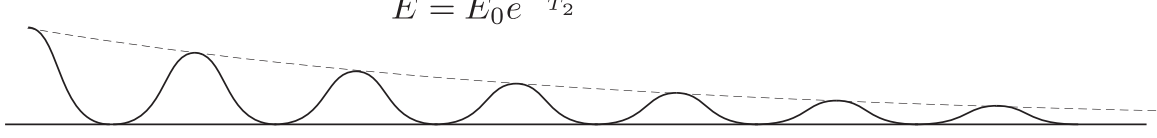

Figure 3.8: A CPMG echo train enables measurement of the transverse relaxation $T_{2}$. The height of each echo is recorded.

$\pi / 2$ pulse a train of equally spaced $\pi$ pulses is used to refocus the magnetisation. This sequence is known by the workers who discovered the necessary phasing of the $\pi$ pulses CMPG. The height of each echo is acquired. The amplitude of successive echoes will diminish exponentially with the characteristic time $T_{2}$. This relaxation is known as spin-spin relaxation and is irrecoverable. A system can exhibit a multiplicity of relaxation times which gives an indication of the local environments of spins.

A typical $T_{2}$ measurement is performed by acquiring the height of each echo and fitting this data to

$$
E(t)=E_{0} \exp \left(-t / T_{2}\right)
$$

where $E_{0}$ describes the amplitude of the initial signal. A distribution of $T_{2}$ in a sample is indicative of differing spins experiencing different environments.

When spins are in the longitudinal axis they will also relax irreversibly with a characteristic time $T_{1}$. This is called spin-lattice or longitudinal relaxation. An example of a pulse sequence used to measure this time is shown in figure 3.9. First the magnetisation is inverted with a $\pi$ and then at increasing delays a $\pi / 2$ pulse transfers what remains in the longitudinal 


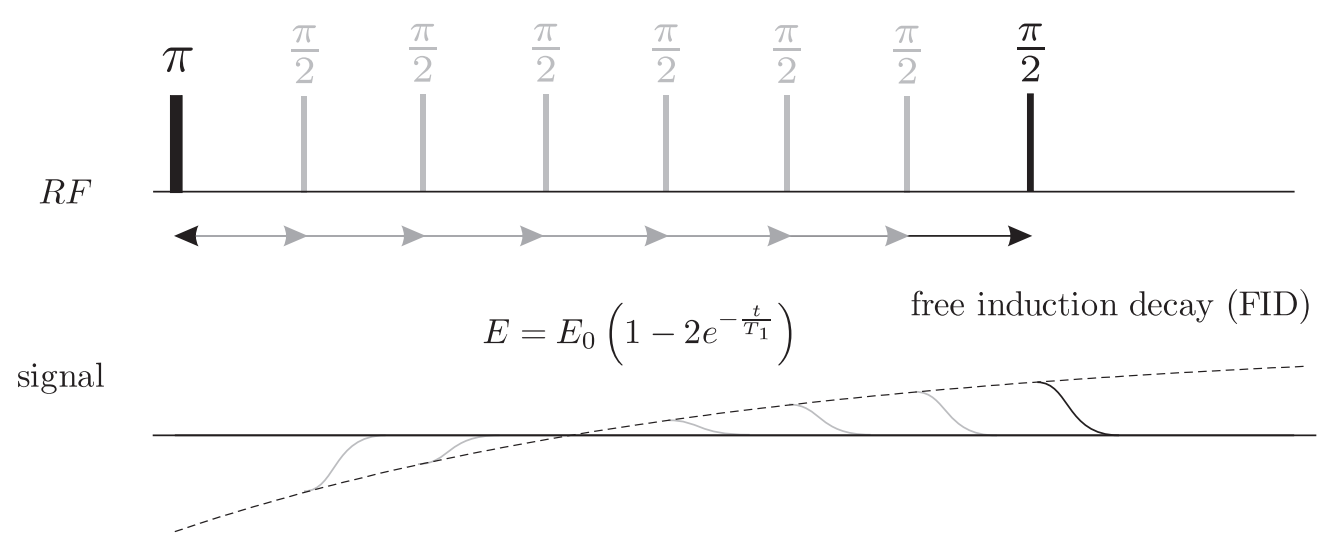

Figure 3.9: A measurement of $T_{1}$ can be made by first inverting the magnetisation with a $\pi$ pulse, and then at successive times measuring how much magnetisation returns to the transverse plane after a $\pi / 2$ pulse. The expression for the time evolution represents a single time constant relaxation from signal inversion to complete recovery.

axis to the transverse plane. The measured data can then be fitted to

$$
E(t)=E_{0}\left(1-2 \exp \left(-t / T_{1}\right)\right)
$$

The longitudinal relaxation is typically slower than the transverse relaxation and less sensitive to the spins local environment. Because $T_{1}$ measures a relaxation back to thermal equilibrium the $T_{1}$ time determines the minimum repetition time between successive scans, ideally a period of $5 T_{1}$ is employed.

\subsection{Spatial Encoding}

The spatial or displacement information in a NMR experiment can be obtained by deliberately perturbing the magnetic field. The superposition of a magnetic field gradient on to $B_{0}$ will result in the spins precessing at slightly different rates, and therefore accumulating phase, dependent on position. For full three-dimensional encoding a three-axis gradient set 
is needed, capable of providing magnetic fields that give the orthogonal gradients

$$
\begin{aligned}
& g_{x}=\frac{\partial B_{z}}{\partial x} \\
& g_{y}=\frac{\partial B_{z}}{\partial y} \\
& g_{z}=\frac{\partial B_{z}}{\partial z} .
\end{aligned}
$$

The fields are produced using electromagnetic coils, standard geometries are given in reference [73].

\subsubsection{One Dimensional Imaging}

A full treatment of imaging will not be given here as this can be found in standard texts $[73 ; 74]$. For a thorough treatment of using NMR to study flow and motion see references [82] and [83]. Pulsed Gradient Spin Echo (PGSE) techniques form the basis of the experiments performed in this thesis and, by way of introduction a one-dimensional, pure phase encoded image will be described.

A one dimensional phase encoded imaging sequence is one of the simplest NMR pulse sequences. The attenuation of an echo is measured for subsequent applications of a pulsed gradient of increasing amplitude. The pulse sequence is shown in figure 3.10. The spatial accumulation of phase, for differing gradient values, is shown in figure 3.11. The construction of the mathematical description of this sequence forms a useful introduction to the sequences that follow.

The signal that is recorded at $2 t_{e}$ will be a summation of the phase from all the spins. With no application of gradient and ignoring $T_{2}$ effects this signal, $E_{0}$, will be proportional to the total number of spins. Typically the acquired signal is normalised by this value. On application of a gradient pulse the spins will acquire a position dependent phase, as shown in figure 3.11. This accumulated phase will be present when the echo is formed, 

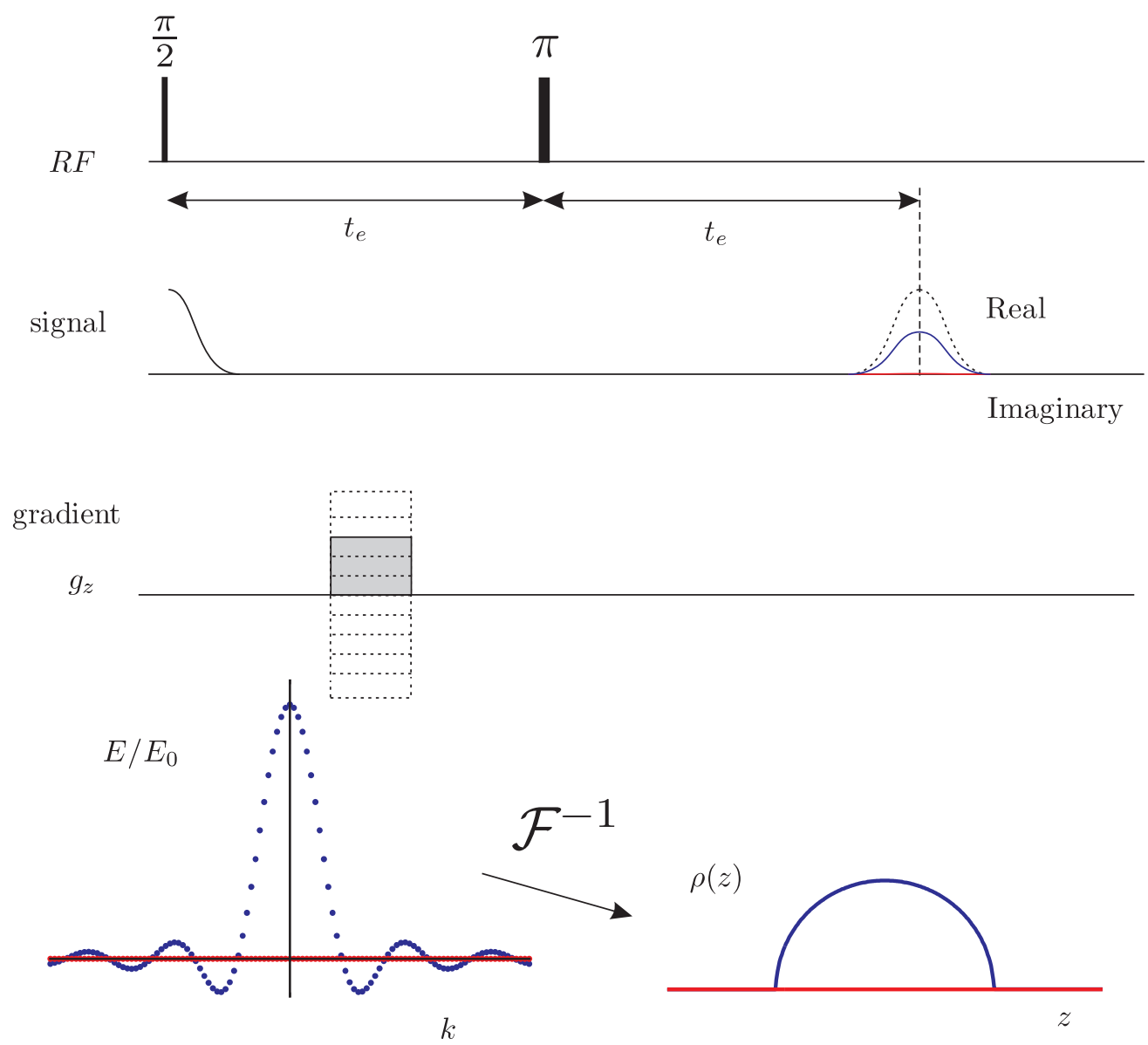

Figure 3.10: The pulse sequence for one dimensional phase encoding. The dotted gradients indicate that the gradient amplitude is stepped. For each gradient value the height of the echo is recorded. Shown is the $k$-space data and one-dimensional image for a cylindrical sample. Each dot in the $k$-space graph represents the measurement of the echo amplitude at a particular gradient value. 


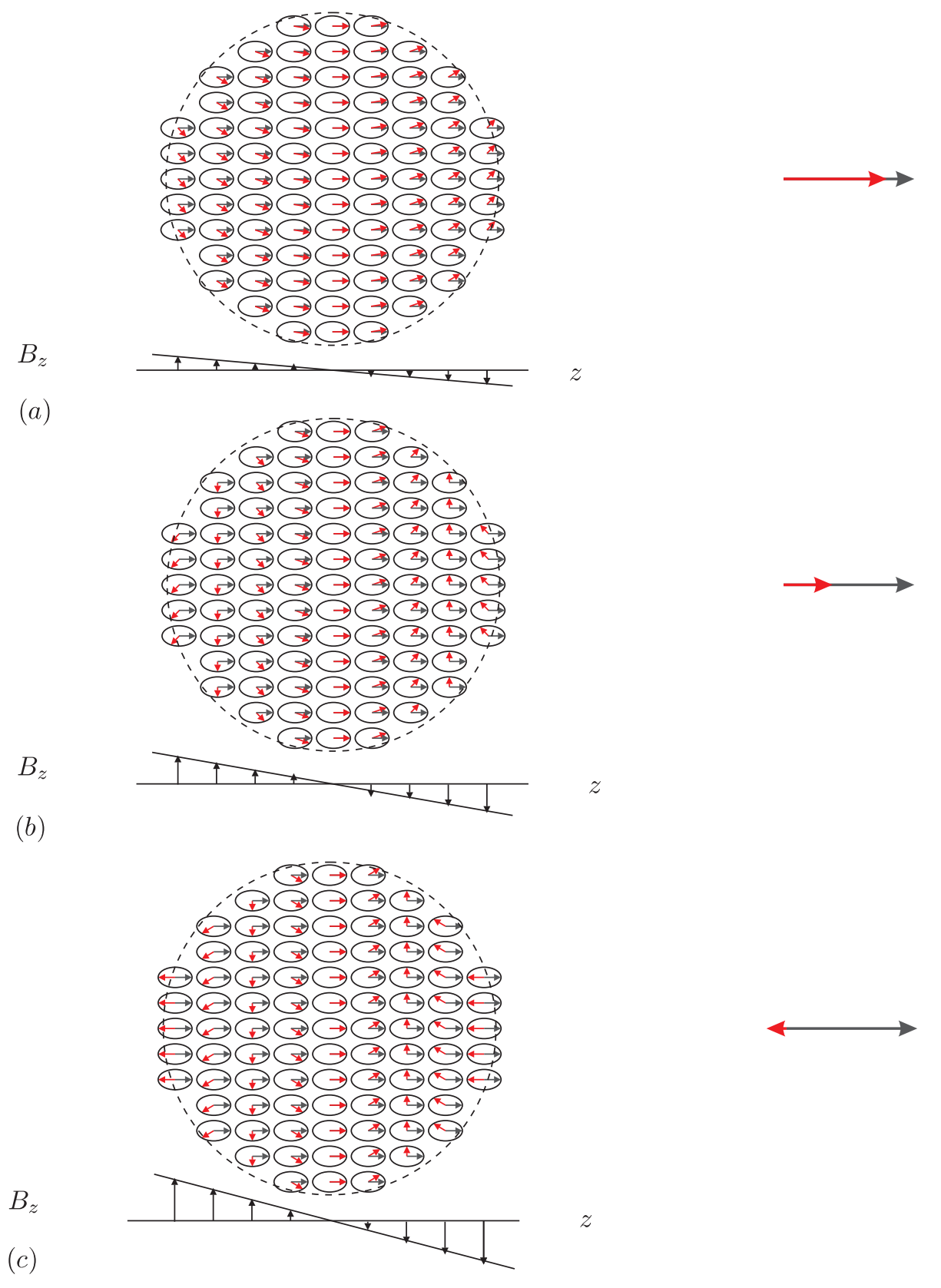

Figure 3.11: The spins in a cylindrical sample will evolve in phase due to the application of a gradient. The initial phase and echo height (on the right) is shown in dark gray, the resulting phase and echo attenuation is shown in red. Panels (a)-(c) show the accumulation and distribution of phase at successively higher gradients. The full $k$-space data is shown in figure 3.10 . 
resulting in a net attenuation of the echo. The spatially dependent phase accumulation is given by

$$
\phi=\gamma \delta \mathbf{g} \cdot \mathbf{r},
$$

in general and following the example in figures 3.10 and 3.11

$$
\phi=\gamma \delta g_{z} z,
$$

where $\delta$ is the length of time for which the gradient pulse is applied. Typically $\pi$ pulses will be around 5-20 $\mu$ s, gradient pulses $0.5-2 \mathrm{~ms}$ and echo times $3-1000 \mathrm{~ms}$. The echo attenuation is then found from summing up all the spatially dependent phase shifts, multiplied by the spin density $\rho(z)$. This can be represented by an integral over all space,

$$
E\left(g_{z}\right)=\int \rho(z) \exp \left(i \gamma \delta g_{z} z\right) d z .
$$

A vector $\mathbf{k}$ can be defined as [84]

$$
\mathbf{k}=\frac{1}{2 \pi} \gamma \delta \mathbf{g},
$$

so that the expression 3.15 can be written as

$$
E\left(k_{z}\right)=\int \rho(z) \exp \left(i 2 \pi k_{z} z\right) d z .
$$

This expression is identical to the definition of the Fourier Transform. If this experiment is performed over all $k$, or at least to a complete attenuation, a numerical inverse Fourier Transform of the echo attenuation with respect to $k_{z}$ will give the spin density $\rho(r)$. This type of imaging is known as phase-encoding. In practice phase-encoding is typically only used for one dimension of an image, the other dimensions provided by a 'read' encoding and from a 'slice' excitation [73].

The echo attenuation as a function of $k$ is a complex quantity and should, in principle, satisfy $E(k)=E^{*}(-k)$. This would result in the measured image being numerically purely 'real'. In complicated imaging sequences 
this is seldom the case due to various artifacts. The image is generally taken as the magnitude such that

$$
\rho(\mathbf{r})=\left|\mathcal{F}^{-1}\left\{E(\mathbf{k}) / E_{0}\right\}\right|
$$

where $\mathcal{F}$ represents a Fourier Transform.

\subsubsection{Displacement Encoding}

In order to measure displacement of spins, a pair of gradient pulses, either side of a $\pi$ pulse, can be used as shown in figure 3.12. A spin that accumulates phase during the first pulse will have the phase fully recovered by the second gradient pulse. However, if the spin has moved during the encoding time $\Delta$ a different phase shift will be applied. This will result in an incomplete rephasing and summing up all of the spins in the sample will give an attenuation and, in the case of flow, a phase shift. This is shown schematically for flow in a cylinder in figure 3.13.

To create an expression for the echo attenuation we need to consider all possible starting positions $\mathbf{r}$ and finishing positions $\mathbf{r}^{\prime}$, and the acquired phase at each position. The chance of finding spins at $\mathbf{r}$ is the spin density $\rho(\mathbf{r})$ and the chance of a spin being at $\mathbf{r}^{\prime}$ after being at $\mathbf{r}$ is the conditional probability $P\left(\mathbf{r} \mid \mathbf{r}^{\prime}, \Delta\right)$. The Echo attenuation can then be written as the integral over all possible starting and finishing positions [85]

$$
E(\mathbf{q})=\iint \rho(\mathbf{r}) \exp (-i 2 \pi \mathbf{q} \cdot \mathbf{r}) P\left(\mathbf{r} \mid \mathbf{r}^{\prime}, \Delta\right) \exp \left(i 2 \pi \mathbf{q} \cdot \mathbf{r}^{\prime}\right) d \mathbf{r} d \mathbf{r}^{\prime}
$$

here, analogous to the definition of $\mathbf{k}$ the gradient product has been replaced by‘[73]

$$
\mathbf{q}=\frac{1}{2 \pi} \gamma \delta \mathbf{g}
$$

The expression 3.19 can be rewritten in terms of displacement $\mathbf{R}$ where 


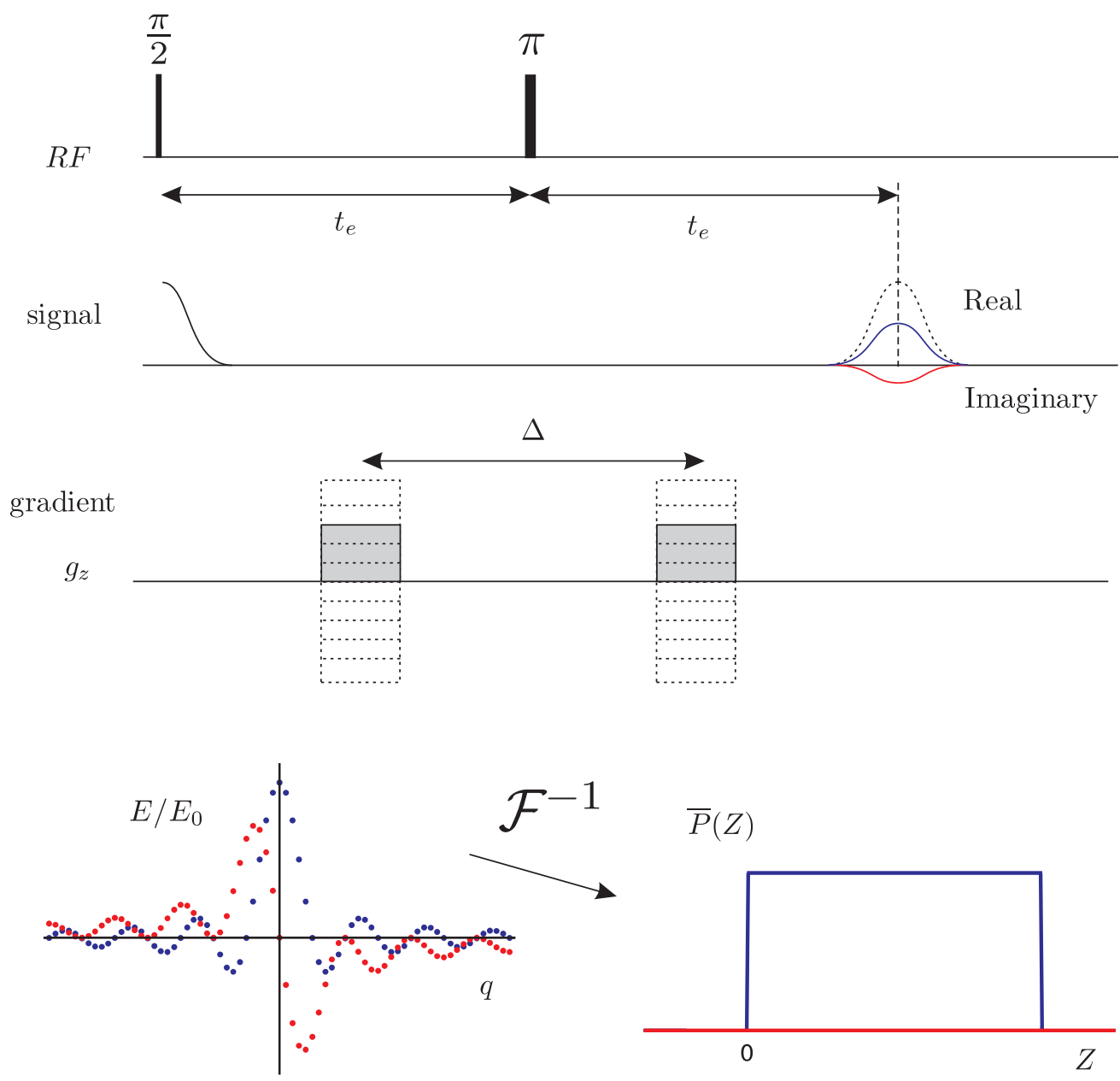

Figure 3.12: The pulse sequence for one dimensional propagator encoding. The echo attenuation and resulting propagator are shown for flow in a cylindrical pipe. Each dot in the $q$-space graph represents the measurement of the echo amplitude at a particular gradient value. 


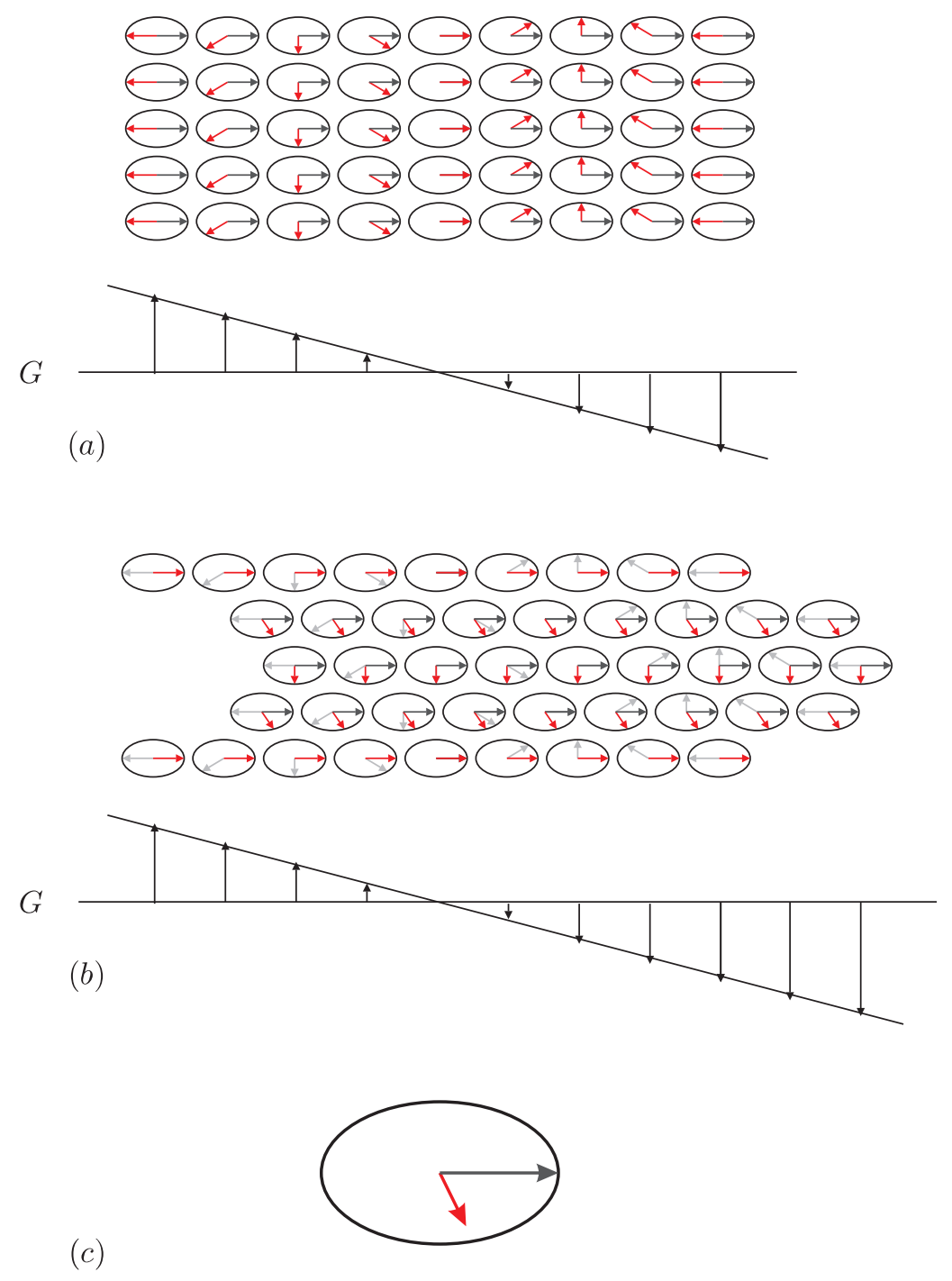

Figure 3.13: The phase changes of flowing spins due to the pulse sequence shown in figure 3.12. Panel (a) shows the initial phase (dark grey) and phase (red) immediately after application of a gradient pulse. This defines the starting time for the propagator encoding. After a $\pi$ pulse and a time $\Delta$ in which the spins have moved, a second, identical gradient pulse is applied. Panel (b) shows the spins phase immediately after the second gradient pulse (red), the phase after the first pulse (light grey) and the initial phase (dark grey). The spins that have moved the furthest have the greatest phase change. The resulting echo, shown in red panel (c), will be attenuated and phase shifted when compared to the case with no gradient (dark grey). 
$\mathbf{R}=\mathbf{r}^{\prime}-\mathbf{r}$. This gives

$$
E(\mathbf{q})=\iint \rho(\mathbf{r}) \exp (-i \mathbf{2} \pi \mathbf{q} \cdot \mathbf{r}) P(\mathbf{r} \mid \mathbf{r}+\mathbf{R}, \Delta) \exp (i 2 \pi \mathbf{q} \cdot(\mathbf{r}+\mathbf{R})) d \mathbf{r} d \mathbf{R}
$$

We can then write this expression so it includes the average propagator [86] $\bar{P}(\mathbf{R}, \Delta)=\int \rho(\mathbf{r}) P(\mathbf{r}+\mathbf{R} \mid \Delta) d \mathbf{r}$, and a single exponential factor

$$
E(\mathbf{q})=\int \bar{P}(\mathbf{R}, \Delta) \exp (i 2 \pi \mathbf{q} \cdot \mathbf{R}) d \mathbf{R}
$$

Similar to the case in the imaging experiment, the inverse Fourier Transform of the echo attenuation will give the average propagator. In an imaging experiment the vector $\mathbf{k}$ describes reciprocal space, in PGSE experiments the vector $\mathbf{q}$ describes reciprocal displacement space. As in an imaging experiment it is preferable to sample $q$-space such that the echo is fully attenuated, this will minimise truncation artifacts. The $q$-space data and a propagator is given for laminar flow in a pipe in figure 3.13 where the encoding direction is longitudinal to the flow. The echo attenuation as a function of $q$ is a complex quantity and should, in principle, satisfy $E(q)=E^{*}(-q)$. In contrast to imaging experiments this is obtainable in propagator measurements. Provided that the phase of $E(q=0)$ is zero, the inverse Fourier Transform should give a propagator that is numerically real, with the imaginary part being close to zero.

\subsubsection{Low-q Approximation}

There are cases when complete attenuation is not desired or not achievable. In this case we can make an approximation to the phase shift to extract the desired information out of the echo attenuation. Beginning with the expression from equation 3.19 we have

$$
E(\mathbf{q})=\iint \rho(\mathbf{r}) \exp (i 2 \pi \mathbf{q} \cdot \mathbf{r}) P\left(\mathbf{r} \mid \mathbf{r}^{\prime}, \tau\right) \exp \left(-i 2 \pi \mathbf{q} \cdot \mathbf{r}^{\prime}\right) d \mathbf{r} d \mathbf{r}^{\prime}
$$


rewriting this using the joint probability gives

$$
E(\mathbf{q})=\iint P\left(\mathbf{r}, \mathbf{r}^{\prime}, \tau\right) \exp \left(i 2 \pi\left(\mathbf{q} \cdot\left(\mathbf{r}-\mathbf{r}^{\prime}\right)\right) d \mathbf{r} d \mathbf{r}^{\prime}\right.
$$

The exponential can be approximated as $e^{x}=1+x+\frac{1}{2} x^{2}+\ldots$ and taking the encoding in the $z$ direction gives

$$
\begin{array}{r}
E(q)=\iint P\left(\mathbf{r}, \mathbf{r}^{\prime}, \tau\right) d \mathbf{r} d \mathbf{r}^{\prime}+i 2 \pi q \iint\left(z-z^{\prime}\right) P\left(\mathbf{r}, \mathbf{r}^{\prime}, \tau\right) d \mathbf{r} d \mathbf{r}^{\prime} \\
-2 \pi^{2} q^{2} \iint\left(z-z^{\prime}\right)^{2} P\left(\mathbf{r}, \mathbf{r}^{\prime}, \tau\right) d \mathbf{r} d \mathbf{r}^{\prime}+\ldots
\end{array}
$$

The first term is, by definition, equal to one and the further terms provide the non-centralised moments of displacement. This expression can then be written as

$$
E(q)=1+i 2 \pi q\langle z(\tau)-z(0)\rangle-2 \pi^{2} q^{2}\left\langle(z(\tau)-z(0))^{2}\right\rangle+\ldots
$$

The notation $\langle\cdots\rangle$ refers to the integral over all starting and finishing positions $\iint \cdots d \mathbf{r} d \mathbf{r}^{\prime}$ and is a form of ensemble averaging. The propagator information is lost in the integral over starting and final position. The full treatment only contains one integral over starting position so the displacement information is retained. A propagator measurement can be thought of as a one-dimensional measurement in terms of the stepped gradients. This results in a one-dimensional quantity, the propagator. A low- $q$ analysis of the same data will result in one or more scalar quantities, in effect losing a dimension. A fit to $q$ of the imaginary part of the echo attenuation in equation 3.26 will give the average displacement and hence displacement. A fit to $q^{2}$ of the real part of equation 3.26 will give the non-centralised second moment of displacement. Taking the magnitude of the echo attenuation will give, using $Z=z-z^{\prime}$

$$
|E(q)|=1-2 \pi^{2} q^{2}\left\langle(Z-\langle Z\rangle)^{2}\right\rangle
$$


where $\left\langle(Z-\langle Z\rangle)^{2}\right\rangle$ is the mean-squared displacement, often written as $\sigma^{2}$. PGSE NMR provides an ideal way to measure the mean squared displacement. The convention of using capital letters to denote displacement will be continued. The analysis is referred to as the 'low- $q$ ' approximation, a more correct name would be the 'low-attenuation' approximation. The approximation used for equation 3.26 is valid for values of $q$ that give an attenuation of around $0.7 E_{0}$.

Measuring mean squared displacement is essential for measuring diffusion and dispersion coefficients, as detailed in section 2.3. For bulk unrestricted diffusion the one dimensional propagator is well known as

$$
P(Z, \Delta)=\frac{1}{\sqrt{4 \pi D \Delta}} \exp \left(-\frac{Z^{2}}{4 D \Delta}\right) .
$$

Substituting this into equation 3.23 gives the natural logarithm of the echo attenuation as

$$
\log _{e} \frac{E}{E_{0}}=-4 \pi^{2} q^{2} \Delta D
$$

For diffusion measurements a correction can be made so that the gradients do not have to be short compared to $\Delta$. This gives the Stjeskal-Tanner relationship [85]

$$
\log _{e} \frac{E}{E_{0}}=-4 \pi^{2} q^{2}(\Delta-\delta / 3) D
$$

where $\delta$ is the length of the gradient pulse. A fit to $q^{2}$ of the logarithm of the echo attenuation can give the diffusion coefficient $D$. This expression does not rely on a low- $q$ analysis.

\subsubsection{Stimulated Echo}

Through the use of a stimulated echo the spins can be stored in the longitudinal axis during encoding periods. This gives access to longer encoding times since typically $T_{2}<T_{1}$. Spins in the transverse plane will also accumulate phase due to background gradients. This unwanted dephasing 
tends to limit the accessible encoding times. In order to minimise the time spent in the transverse plane a stimulated echo can be used. This is where the magnetisation is effectively stored along the $z$-axis during the encoding interval. The pulse sequence is shown in figure 3.14. An initial $\pi / 2$ pulse is followed by the first encoding gradient. After this a $\pi / 2$ 'storage pulse' is used to store the magnetisation in the $z$-axis. During the storage period a 'crusher' gradient is used to make sure any magnetisation remaining in the transverse plane is dephased. The magnetisation is then 'recalled' by a further $\pi / 2$ pulse, a second encoding gradient is applied and an echo is formed.

The dephasing of the transverse magnetisation during the storage time means that the storage and recall $\pi / 2$ pulses can not be considered purely as a $\pi$ pulse. As shown in figure $3.15(b)$ the initial gradient pulse produces a distribution of phase angles in the transverse plane and the storage $(\pi / 2)_{x}$ pulse rotates this distribution into the $x-z$ plane, figure 3.15(c). During the storage time the $y$ component of the magnetisation is lost, figure 3.15(d). In order to correctly preserve the entire encoding a phase cycle must be used to store and recall the complete components of the phase. Figure 3.15(g)-(l) shows the second half of the phase cycle that gives the complete phase encodings. The superposition of figures 3.15(f) and 3.15(l) shows that all phase encoding will be preserved. This is essential for experiments where there is a $q$ dependent phase shift due to some bulk flow. For diffusion measurements this is unnecessary, except for very low attenuation. A phase cycle is also necessary to ensure correct encoding, compensated for receiver offsets and to minimise unwanted signal from RF pulses.

The phase cycle typically used for a stimulated echo experiment, known as a PGSTE (pulsed gradient stimulated echo), is given in table 3.1. Note the the phase of the receiver channel is also cycled thus eliminating any inherent DC offset. The resulting signal is reduced by a factor of 2 per scan, when compared to a standard PGSE experiment. 


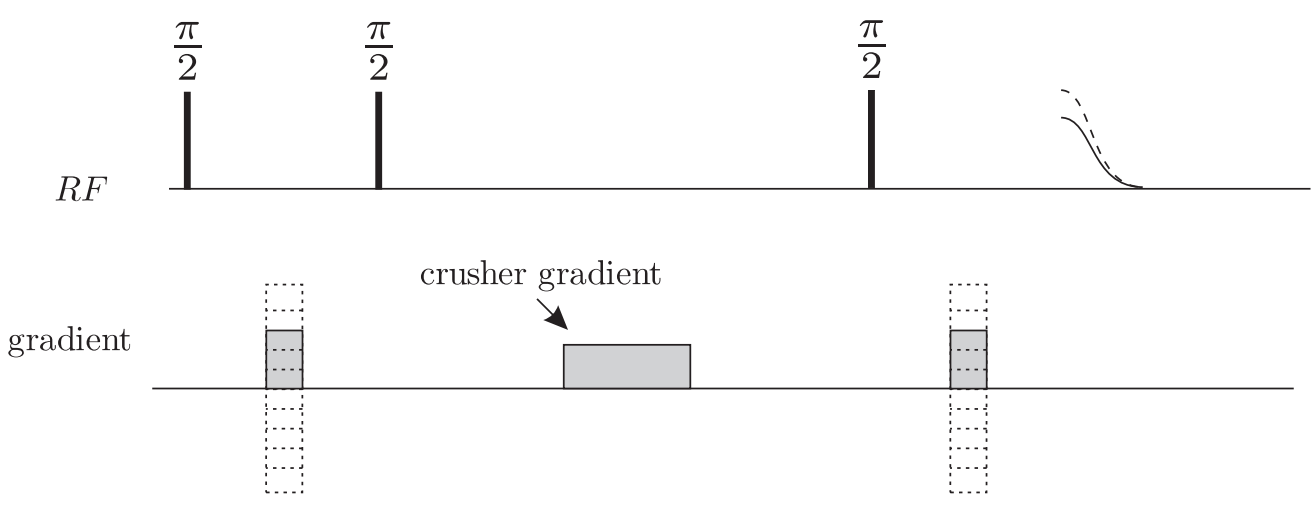

Figure 3.14: In a stimulated echo sequence the gradient pulses, which are separated by $\Delta$ provide the displacement encoding. During this encoding period the magnetisation is 'stored' in the $z$-axis to reduce $T_{2}$ losses and background gradient effects. A crusher gradient is used to ensure the loss of transverse magnetisation.

\begin{tabular}{|c|rrrr|}
\hline Excitation & $\mathrm{x}$ & $\mathrm{x}$ & $\mathrm{x}(-\mathrm{x})$ & $\mathrm{x}$ \\
Storage & $\mathrm{x}$ & $-\mathrm{x}$ & $\mathrm{y}(-\mathrm{y})$ & $-\mathrm{y}$ \\
Recall & $\mathrm{x}$ & $-\mathrm{x}$ & $\mathrm{y}(-\mathrm{y})$ & $-\mathrm{y}$ \\
Receiver & $\mathrm{x}$ & $\mathrm{x}$ & $-\mathrm{x}(\mathrm{x})$ & $-\mathrm{x}$ \\
\hline
\end{tabular}

Table 3.1: The 4-step phase cycle for the pulse sequence depicted in figure 3.14. This phase cycle minimises unwanted signals and also ensures pure phaseencoded terms after recall following the storage period. Columns 1 and 3 (in parenthesis) describe the phase cycle depicted in fig 3.15. In practice the phase cycle also needs to cycle through the receiver (Columns 3 and 4) and minimise unwanted FIDs from the recall $\pi / 2$ pulse (Columns 2 and 4 ) 


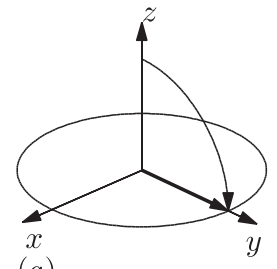

$(a)$
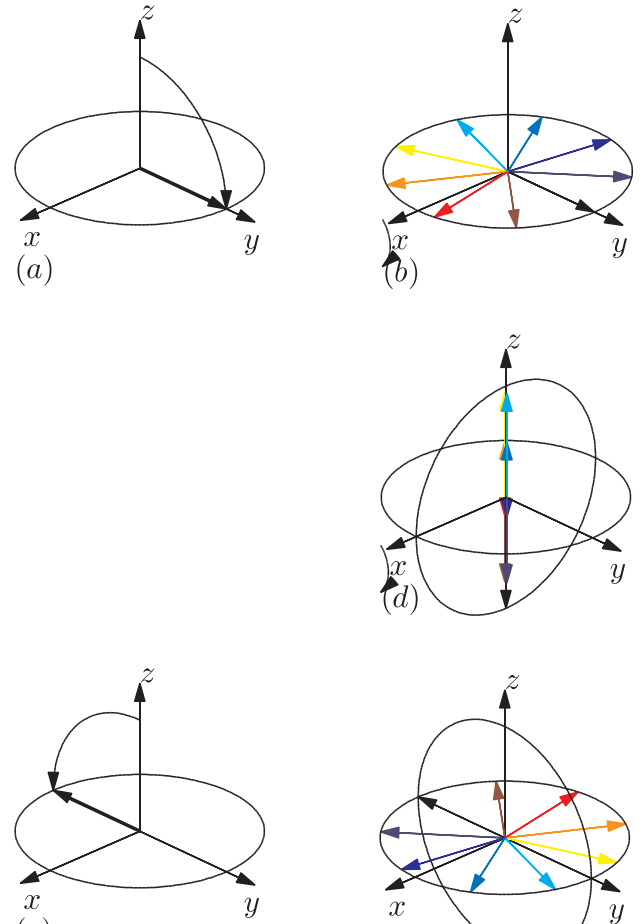

$(g)$
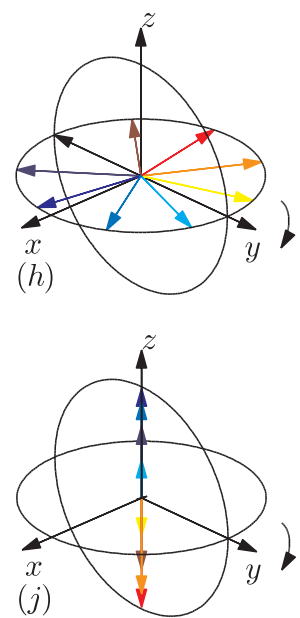
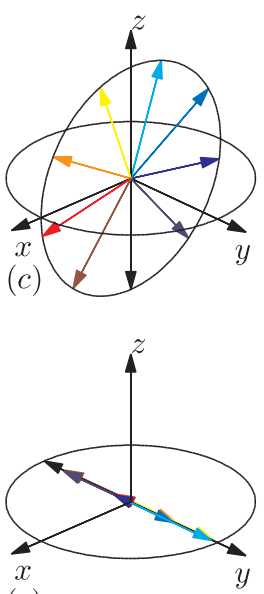

(e)
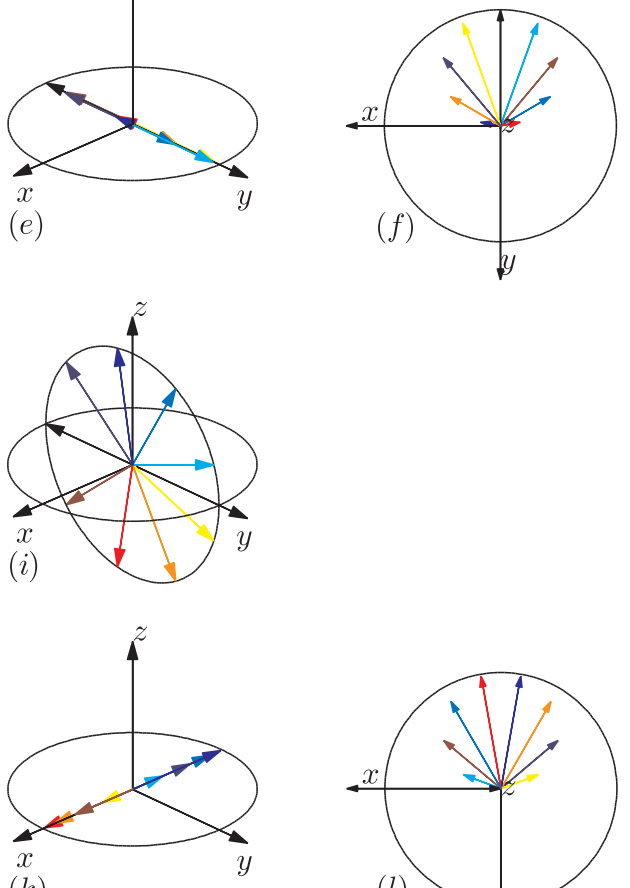

$(k)$

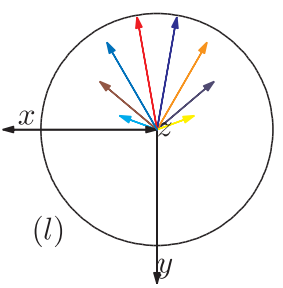

Figure 3.15: The storage and recall of magnetisation causes loss of the phase of some spins. Panels (a)-(f) show the first half of the phase cycles. The echo is formed (for no net encoding) in panel (f) showing spins with incomplete phase information. To preserve the complete encoding the second component of the magnetisation distribution must be stored in the $z$-axis. ((g)-(l)). The superposition of (f) and (I) shows the complete refocussing of all the phase information. 


\subsubsection{Double PGSTE}

A double PGSTE experiment is used to encode for displacement and then encode for displacement after a mixing time $\tau$. Such pulse sequences have been used to measure the velocity autocorrelation function (VACF). The sequence is shown in figure 3.16(a). The initial and final encoding for displacement are shown as Hahn echoes whereas the mixing time is encoded with a stimulated echo. This pulse sequence is typically performed and analysed in the low- $q$ limit.

The double PGSTE experiment requires some phase cycling to eliminate unwanted coherences [87]. Spins that have relaxed back to thermal equilibrium or moved into the sensitive volume will see a pulse sequence that is not intended. Shown in figure 3.16(b) is the first $\pi$ pulse effectively acting as a excitation pulse. This will be seen by a small amount of spins that have relaxed back to thermal equilibrium since the initial excitation. This unwanted magnetisation will then be stored in the $z$-axis and may be recalled not by the $\pi / 2$ pulse but by the second $\pi$ pulse acting as a $\pi / 2$ pulse. Note that in this case dephasing due to the gradients will be refocussed. Figure 3.16(c) shows the recall $\pi / 2$ pulse acting as an initial excitation pulse for all the spins that have relaxed back to thermal equilibrium since the initial excitation. The gradients and the last $\pi$ pulse provide an unwanted echo. Both of these coherences can be minimised through cycling the phase of the RF pulse so that the desired signal continues to superpose while the unwanted signal is cancelled out. Further details are given in section 5.4.1 and table 5.1.

A double PGSTE experiment is typically implemented with the initial and final encoding times $\Delta_{u}$ to be as short as possible. If this time is short compared to some characteristic time of the flow, the encoding can be considered as a velocity. A double PGSTE experiment can be implemented as a two dimensional experiment where the gradients are stepped independently. If the measurement is performed to full attenuation a Fourier Transform will then recover a two-dimensional probability distribution 

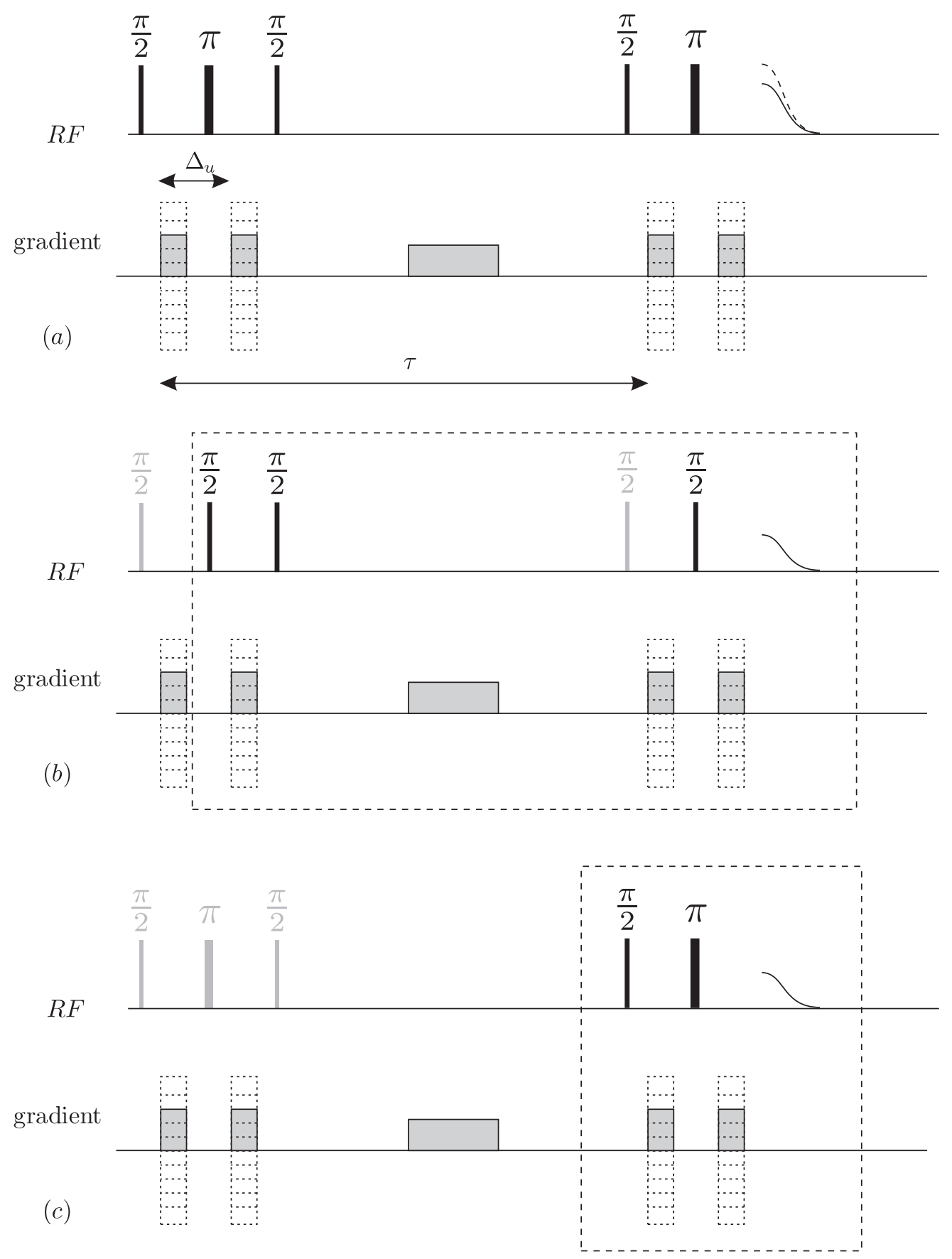

Figure 3.16: A double PGSTE is shown in panel (a). The encoding time $\tau$ is a stimulated echo but the encoding for initial and final displacement is a normal echo. Panels (b) and (c) show two of the unwanted coherences that need to be cycled out, see text for dicussion. 
$\bar{P}\left(V_{z}(0), V_{z}(\tau), \tau\right)$ which describes the velocity exchange of particles [88].

If the gradients of a double PGSTE are stepped in unison there are two configurations. If the gradients are stepped as in figure 3.16(a) the phase shift due to the bulk flow is cancelled out and is thus called a 'compensated' double PGSTE [16]. In a low- $q$ analysis, and treating the displacement between the pairs of pulse as $\mathbf{v} \Delta_{u}$, we can write, with the encoding in the $z$ direction

$$
E(q)=\left\langle\exp \left(i 2 \pi q_{z} \Delta_{u} v(0)\right) \exp \left(-i 2 \pi q_{z} \Delta_{u} v(\tau)\right)\right\rangle .
$$

Rewriting the velocity as $u=v-\langle v\rangle$ gives

$$
E(q)=\left\langle\exp \left(i 2 \pi q_{z} \Delta_{u} u(0)\right) \exp \left(-i 2 \pi q_{z} \Delta_{u} u(\tau)\right)\right\rangle
$$

here there is no dependence on $\bar{v}$ thus showing the compensating nature of the second gradient pair. Approximating the exponentials gives

$$
\left.E(q)=\left\langle\left(1+i 2 \pi q_{z} \Delta_{u} u(0)-2 \pi q_{z} \Delta_{u} u(0)\right)\left(1-i 2 \pi q_{z} \Delta_{u} u(\tau)-2 \pi q_{z} \Delta_{u} u(\tau)\right)\right)\right\rangle
$$

which is, to $q^{2}$

$$
E(q)=1-4 \pi^{2} q_{z}^{2} \Delta_{u}^{2}\left(\left\langle u^{2}\right\rangle-\langle u(0) u(\tau)\rangle\right)
$$

since the second moment of velocity $\left\langle u^{2}\right\rangle$ is independent of time. A fit to $q^{2}$ of equation 3.34 can provide access to $\left(\left\langle u^{2}\right\rangle-\langle u(0) u(\tau)\rangle\right)$ from which the velocity autocorrelation can be recovered provided a measurement of $\left\langle u^{2}\right\rangle$ can be made, typically at a long encoding time $\tau$ when the VACF is taken to be zero [16]. Such an approach presupposes the existence of asymptotic conditions. In chapter 5, through the use of a second uncompensated double PGSTE, the VACF can be recovered independently of an infinite time encoding. This technique forms a crucial part of the measurement detailed in this thesis. 

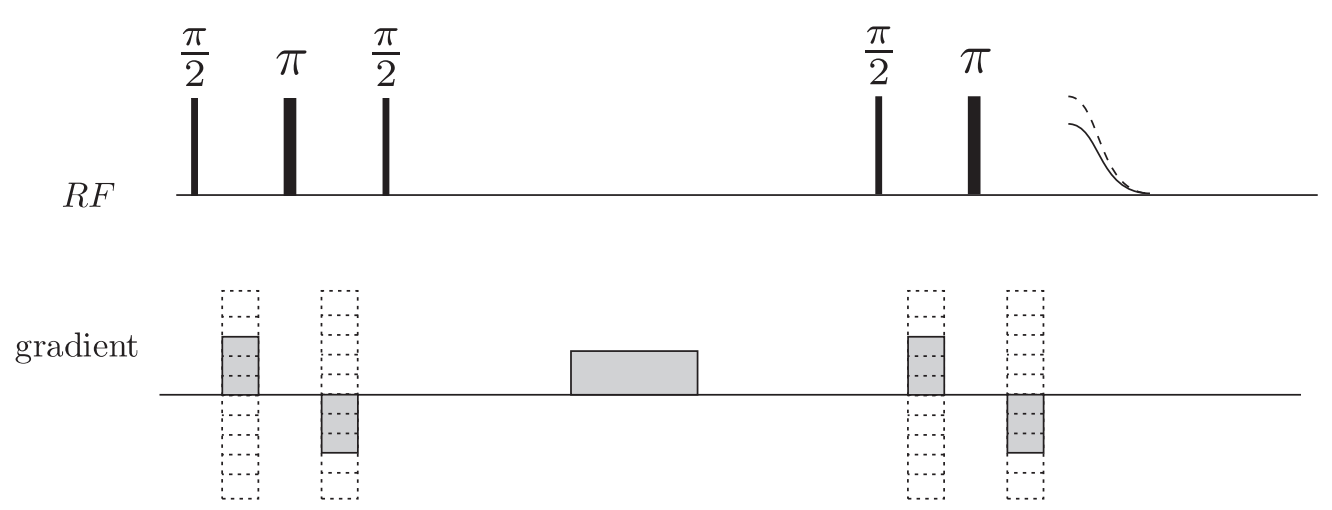

Figure 3.17: A 'Cotts' or 'Bi Polar' PGSE. This implementation of a PGSTE experiment is designed to minimise unwanted attenuation from static, internal gradients.

\subsubsection{Bi Polar PGSE}

When PGSE experiments are performed on porous media the internal gradients due to a susceptibility mismatch provide an additional unwanted dephasing. To minimise this effect a 'Cotts' sequence [89] can be used. The time in which the initial gradient pulse is applied is divided into two by the application of a $\pi$ pulse. This ensures that the dephasing due to the background gradients is at least partially refocussed. The gradient pulse itself is divided into the two intervals, the $\pi$ pulse requiring that each half of the gradient pulse be implemented with opposite polarity (see figure 3.17). Ideally the gradient pulses should be equidistant between the RF pulses. The second rephasing gradient pair is applied similarly. Note that the RF scheme for this pulse sequence is identical to the double PGSTE shown in figure 3.9(a).

\subsection{Dispersion and NMR}

Some of the NMR applications in characterising porous media are summarised in chapter 2 . Here a brief review of NMR techniques specifically 
related to dispersion measurements is given.

Some of the early measurements using NMR for studying dispersion in porous media used imaging techniques to measure the displacement of oil in a rock sample [90]. Guillot et al. used imaging techniques to measure the effect of paramagnetic tracer particles [91; 92], a dispersion coefficient was fitted to the resulting concentration profiles. Propagator measurements using PGSE and PGSTE NMR and analysis of the propagator shape in a beadpack was investigated by Lebon et al. in [93-95].

The first measurements of a dispersion coefficient using PGSE NMR to measure mean-squared displacement were reported simultaneously in 1996 by Seymour and Callaghan [53] and by Ding and Candala [24]. The former paper also investigated 'flow diffraction' through $q$-space imaging. The latter introduced the notion of measuring nonlocal dispersion using NMR. Measurements of longitudinal and transverse dispersion coefficients were given by [96] and [97], the latter containing comparisons to preexisting standard techniques for measuring dispersion. A second paper by Seymour and Callaghan in 1997 [14] demonstrated the unique capabilities of PGSE NMR in relation to all aspects of dispersion and has become a 'standard text' in the field. In 1999 the same authors with Codd and Manz [98] performed NMR dispersion measurements in a capillary (see section 6.2) which were able to be compared directly with theory.

Extensive dispersion measurements of chromatography columns were made by Tellarek et al. [99-101]. Comparisons were made between results from NMR measurements and other measurement techniques.

In 1998 Manz et al. measured and modelled flow and dispersion using NMR and the lattice Boltzmann technique [22]. This paper represents an important link between simulation and experiments and is covered, with other simulations in the literature, in section 4.3. Further investigations into spatial correlations of dispersion were investigated [15; 23]. Many other studies of flow and structure in porous media and beadpacks have used MRI velocimetry techniques [102-104]. 
The double PGSE experiment, already proposed as a VESXY experiment in 1994 [88], was further shown in 1999 to be able to differentiate between the stochastic and deterministic components of the flow [105]. Extensive use of the double PGSE experiment was used by Khrapitchev et al. to measure the velocity autocorrelation function [16]. Double PGSE techniques have been used recently for further diffusive characterisation of porous media [106-108].

In 1995 Callaghan and Stepisnik began investigations of dispersion in the frequency domain rather than time [109]. These experiments are performed with trains of gradient pulses. These flow coherences where further investigated in [110-112]. In 2000 Stapf et al. used a two-dimensional PGSE sequence, accompanied by numerical simulations, to measure twodimensional flow propagators in beadpacks and rocks [21].

The work by Scheven et al. has investigated the preasymptotic dispersion regime to make quantitative measurements [113; 114], and quantify the effect of relaxation in inhomogeneous field in propagators [29]. Flow and relaxation effects were measured by Britton et al. [115] earlier in 2001, and further extended by Mitchell et al. [116] in 2008.

Dispersion measurements in NMR have been made using gas as a probe fluid, thermally polarised propane [117] and hyperpolarised Xenon [118120]. A gas as a fluid probe provides access to larger diffusive length scales. Thermally polarised gasses enable the use of conventional NMR techniques while hyperpolarised gasses provide a signal-to-noise enhancement.

A novel propagator study with encoding times of 35s was achieved in [121] using a polarisation transfer to a ${ }^{13} \mathrm{C}$. While this represents an order of magnitude increase in the measurement time scale, a limitation of this technique is the requirement for hydrocarbon-based solvents or probe fluids. An improvement to the experimental techniques of measuring of propagators has been described in [122; 123]. A sequence called 'difftrain' uses a stimulated echo with a partial recall to enable propagator measure- 
ments at multiple encoding times.

\subsubsection{Ensemble Averaging}

Undoubtedly the use of MRI velocimetry has given great insight into the flow and transport properties of porous media. However, the length scales that can be probed using imaging techniques are inherently limited by signal-to-noise. Spatially encoded images necessarily contain little information about flow coherences over large distances. Pulse gradient spin echo techniques are ensemble averaged techniques such that signal from parts of a sample that are probing short distances are averaged together. This bulk measurement enables probing of distances that are far shorter than the smallest voxel achievable with MRI [14; 17]. Ensemble averaged techniques are therefore preferred for investigations of correlation and small scale displacement effects.

\subsection{Technical Aspects of Applying Gradients}

Large $q$ values are required to probe short displacements in PGSE experiments. Having a large $g$ enables the time the gradient is switched on $\delta$ to be minimised. This is useful so that the time in the transverse plane can be minimised and so that smaller encoding times can be measured. The maximum gradient achieved and the shortest $\delta$ is limited by hardware. The experiments described in this thesis utilised a Bruker three-axis mirco-imaging gradient set. Current supplies capable of pulsing $60 \mathrm{~A}$ enabled a maximum gradient of $1.45 \mathrm{~T} / \mathrm{m}$.

The non-zero inductance of the gradient coils results in the current amplifiers responding to a square pulse with a characteristic rise time. The rise time can be minimised by adjusting the impedance of the feedback loop in the current amplifier. To enable the current supply to more accurately reproduce the desired current pulse shape a trapezoidal gradient 


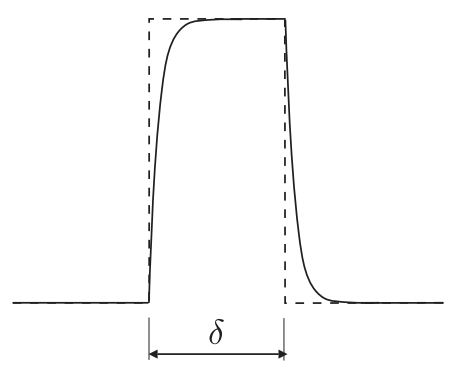

(a)

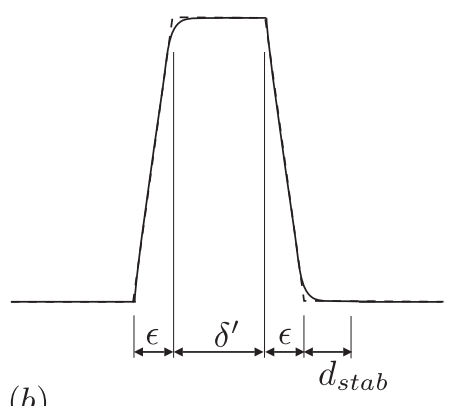

(b)

Figure 3.18: A typical amplifier response to a rectangular gradient pulse is shown in (a). In order for the actual current pulse to be a better match for what is desired a trapezoidal pulse is used (b). The effective length of the pulse can be given as $\delta=\delta^{\prime}+\epsilon$

pulse is typically used. This is shown schematically in figure 3.18. Typically the length of the gradient pulse is 500-1000 $\mu$ s and the ramp time, $\epsilon$, is $100 \mu \mathrm{s}$. The effective length of the gradient pulse is $\delta^{\prime}+\epsilon$, hereafter we refer to this effective time as $\delta$.

Subsequent to the application of a large current pulse a small stabilisation delay, $d_{\text {stab }}$, is needed. A long tail switch off current will result in a magnetic field gradient and it is preferable to allow this to stabilise before applying an RF pulse. Typically $500 \mu$ s is used.

The change in magnetic field from the gradient coils can induce eddy currents in the bore of the magnet that result in an unwanted time-dependent magnetic field. This will result in a small $q$ dependent phase shift of the echo attenuation. Although this effect is minimised by designing actively shielded gradient coils a small effect sometimes remains. For sensitive measurements of flow, the measured $q$-dependent phase shift can be compared to that of the same experiment with zero flow.

In order to obtain echo attenuation that is solely due to small particle displacements, the gradient pulses need be very well matched. In most amplifiers a slight drop between subsequent pulses will occur due to the 


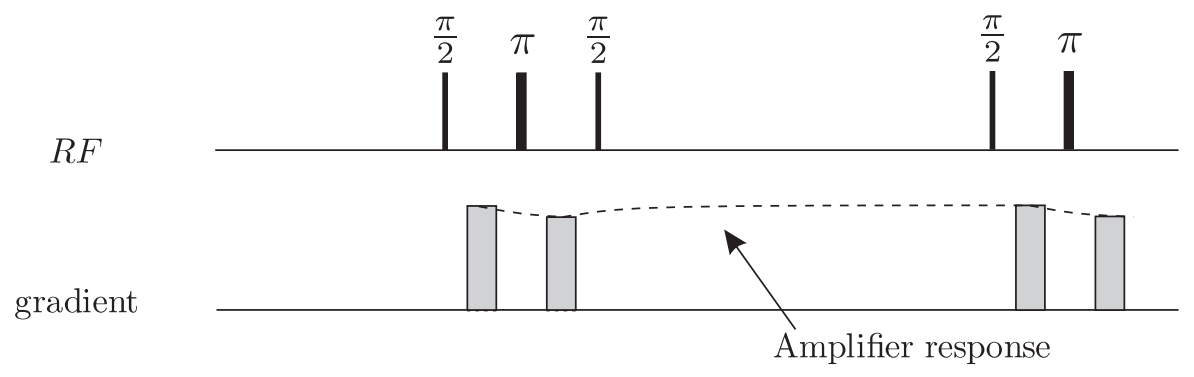

$(a)$

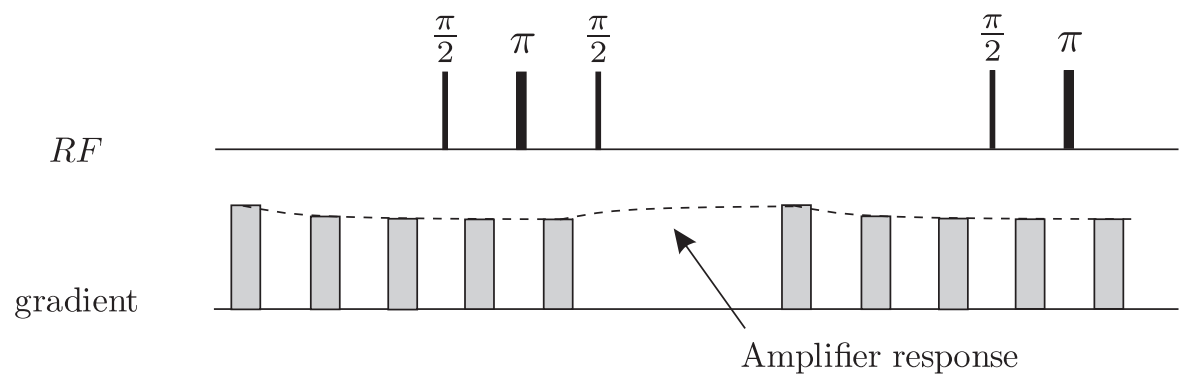

(b)

Figure 3.19: Panel (a) shows the drop in amplitude to gradient pulses due to the amplifier response. In panel (b) a train of 'warm-up' gradient pulses is used to saturate the amplifier response to ensure evenly matched gradient pulses 
finite responsiveness. To ensure well matched gradient pulses, a 'warmup' train of pulses is used to force the amplifier into equilibrium. This can be done, for a double PGSTE, before the initial encoding and then again before the recall $\pi / 2$ pulse as shown in figure 3.19. The loss in amplitude is exaggerated and is, in practice, insignificant when compared to the improvements made by having well matched gradient pulses.

\subsection{Conclusions}

A basic introduction has been given to the phenomenon of nuclear magnetic resonance. Particular attention has been given to pulsed gradient spin echo methods for measuring flow and displacements. These methods are important to the methodology development described further in chapter 5 where we will see that through a combination of PGSTE and double PGSTE experiments, nuclear magnetic resonance is capable of measuring a unique quantity, as introduced in section 2.4, the nonlocal dispersion tensor. 


\section{Chapter 4}

\section{Simulation of Dispersion in Porous Media}

\subsection{Introduction}

The work in developing the simulation code was performed by people other than the author, the majority by Andrew Jackson with contributions in the early phase from Brett Ryland and Ed Crossland. This project has made extensive use of the simulation suite and as such, some relevant details and background are included here.

The simulation suite is based around a lattice Boltzmann calculation of a low Reynolds number flow field. This simulation requires a description of the medium, in our case a random bead pack, on an evenly spaced grid. Finally, to simulate the dispersion a 'tracer particle' simulation is used. Large numbers of tracer particles are allowed to flow and diffuse through the flow field, the position and velocity of each particle being determined at arbitrary intervals.

An introduction is given to the components of the simulation suite along with relevant background information. Also included is a brief review of other research groups' use of lattice Boltzmann to model behaviour in porous media. Due to the size of this research field, we restrict 
the focus to comparison with NMR measurements and dispersion calculations via tracer particle simulation. For a thorough review of lattice Boltzmann simulations the reader is referred to standard texts such as [124;125] and recent reviews focussed on flow in porous media [126; 127].

\subsection{The Simulation Suite}

\subsubsection{Lattice Boltzmann Calculation of the Flow Field}

The complex boundaries in porous media have been thought to cause conventional computational fluid dynamics simulation to become numerically unstable [128]. However several researchers have simulated flow in bead packs with low bead to cylinder ratios of 2-4 [42] and more recently with ratios of 5 [43] and 10 [44]. Nevertheless the widespread use of lattice Boltzmann simulations in comparison with NMR measurements, and the ability to develop an 'in-house' simulation suite meant that a lattice Boltzmann based solution was preferred.

Lattice Boltzmann methods (LBM) for simulating hydrodynamics appeared in the late 1980s [129-131]. The technique has its origins in latticegas cellular automata which can give solutions to the Navier-Stokes equation [132]. In 1997 it was demonstrated that the lattice Boltzmann equation can be found directly from discretising the Boltzmann equation [133; 134].

Lattice Boltzmann simulations have become increasingly popular for the study of flow in porous media, as the algorithm is able to be implemented so as to take advantage of parallel processing so that the complicated boundaries and pore space do not significantly increase computational time [125]. The majority of lattice Boltzmann simulations are performed on parallel machines [135]. However, recently it has been demonstrated that medium sized grids can be simulated on a desktop PC [136]. That is the basis of the simulations in this work.

A lattice Boltzmann simulation represents a class of cellular automata 

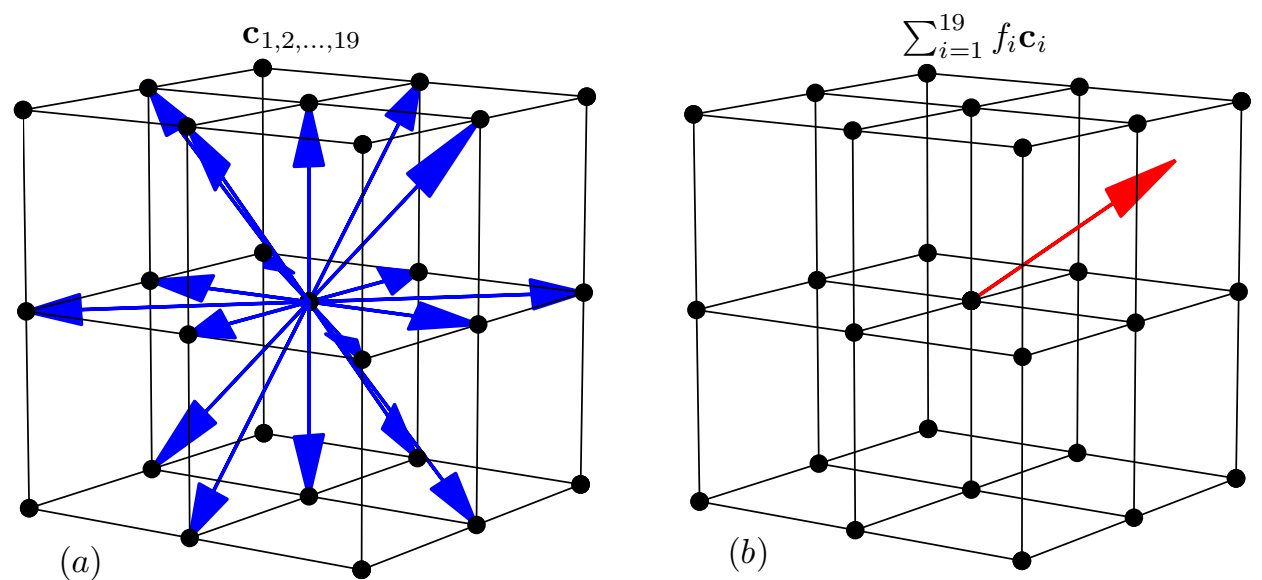

Figure 4.1: Panel (a) shows the 19 allowed velocities. There is one zero velocity vector, for a stationary velocity step, 6 velocities to the nearest neighbour (the center of the face) and 12 velocities to the next-nearest neighbour (the cube edges). Note the velocities to the corner lattice sites are not permitted. Panel (b) shows the sum of these 19 velocities weighted by the probability distribution $f_{i}$ to give the velocity $\mathbf{v}$ at this lattice site.

with one important difference. Instead of each lattice site being populated with a single particle, it is populated with a distribution of velocities. For each lattice site there is a set of velocity vectors $\mathbf{c}_{i}$ that represent a velocity step directly to the next lattice site. Differing implementations of lattice Boltzmann simulations use different definitions for this set. We use a D3Q19 lattice (see figure 4.1(a)). This notation refers to a 3 dimensional lattice with 19 velocity vectors. These 19 velocity vectors consist of steps to the 6 nearest neighbours, the 12 next-nearest neighbours and the stationary vector. Each lattice site has a distribution function $f_{i}$ that describes the proportion of each velocity vector $\mathbf{c}_{i}$. In this way the velocity at each lattice site can be found as

$$
\mathbf{v}=\frac{\sum_{i}^{19} f_{i}(x, t) \mathbf{c}_{i}}{\rho(x, t)}
$$


where the density $\rho(x, t)$ is given as

$$
\rho(x, t)=\sum_{i}^{19} f_{i}(x, t) .
$$

The discretisation of the Boltzmann equation can be written as, for a lattice size of $\Delta x=1$ and a simulation time step of $\Delta t=1$,

$$
f_{i}\left(\mathbf{x}+\mathbf{c}_{i} \Delta t, t+\Delta t\right)-f_{i}(\mathbf{x}, t)=\Omega_{i}(f(\mathbf{x}, t)), \quad(i=1,2, \ldots, 19),
$$

where $\Omega_{i}$ is the collision operator, which can take on different forms. The most common is the Bhatnaga-Gross-Krook (BGK) operator [137], first suggested for lattice Boltzmann methods in [138]. Here the particle distribution after propagation is relaxed towards the so-called equilibrium distribution $f_{i}^{e q}(\mathbf{x}, t)$ as

$$
\Omega_{i}=\frac{1}{\tau}\left(f_{i}(\mathbf{x}, t)-f_{i}^{e q}(\mathbf{x}, t)\right) .
$$

where the parameter $\tau$ describes the relaxation and is usually of the order of 1 . The equilibrium density is found as

$$
f_{i}^{e q}=w_{i} \rho\left(1+\frac{\mathbf{c}_{\mathbf{i} \cdot \mathbf{v}}}{c_{s}^{2}}+\frac{\left(\mathbf{c}_{\mathbf{i}} \cdot \mathbf{v}\right)^{2}}{c_{s}^{4}}-\frac{\mathbf{v} \cdot \mathbf{v}}{2 c_{s}^{2}}\right)
$$

where $c_{s}$ is the speed of sound in simulation units. This enables recovery of the Navier-Stokes equation in the low Mach number limit [139], giving $c_{s}$ as $\sqrt{1 / 3}$ and the kinematic viscosity $\nu=(2 \tau-1) / 6$. The coefficients $w_{i}$ in equation 4.5 are $1 / 3$ for the rest population, $1 / 18$ for the population moving to the nearest lattice sites and $1 / 36$ for the population moving to the next-nearest lattice sites (See figure 4.1). The BGK algorithm is a single relaxation time operator. It has been shown that multiple time relaxation operators are more accurate [126] in certain cases.

The flow is found iteratively by calculating the equilibrium distribution $f_{i}^{e q}$ from equation 4.5 from some initial (or current) flow field using 
the definition from equation 4.1. The next iteration of the distribution can be found be from the propagation

$$
f_{i}\left(\mathbf{x}+\mathbf{c}_{i} \Delta t, t+\Delta t\right)=f_{i}(\mathbf{x}, t)+\frac{\Delta t}{\tau}\left(f_{i}(\mathbf{x}, t)-f_{i}^{e q}(\mathbf{x}, t)\right)+\rho \frac{\Delta t}{c_{s}^{2}} \mathbf{g} \cdot \mathbf{c}_{i} .
$$

The pressure gradient is specified via a bulk force, $g$, in our case along the $z$ direction. This has been shown to be equivalent to a uniform pressure gradient [140].

Any propagation of $f$ from a fluid site to a solid site is directly reflected back onto the original site. Note this is a different phenomenon to the 'bouncing' of diffusing particles. The reflection effectively places the boundary mid way between the lattice sites and ensures a no-slip boundary condition. This has been shown to be accurate to second-order [141]. Other bounce back schemes are available [126] but are not implemented here.

The typical lattice size of our simulations is $105 \times 105 \times 205$. The algorithm is run, with a $\tau$ of 0.571 , until steady state velocities are achieved. This is typically after 10,000-20,000 iterations, a few hours on a desktop PC. Figure 4.2 shows a slice though a randomly generated bead pack (see section 4.2.2). The colourmap relates to the magnitude of the velocity.

The dimensional scaling factors are; for length units, $L / N$, where $L$ is the length of the lattice in physical units and $N$ is the number of lattice sites, for time units, $\frac{c_{s} L}{C_{s} N}$ where $C_{s}$ is the speed of sound in physical units.

\subsubsection{Beadpack and Lattice Generation}

The algorithm for generating the beadpack is based on that of [142] and the 'ballistic' model of [143]. The beadpack is established, bead-by-bead, using a discrete random-walk to move each sphere's centre around within the confining cylinder. To simplify the construction process, this random walk is constrained so that the sphere centers always lie upon nodes of 


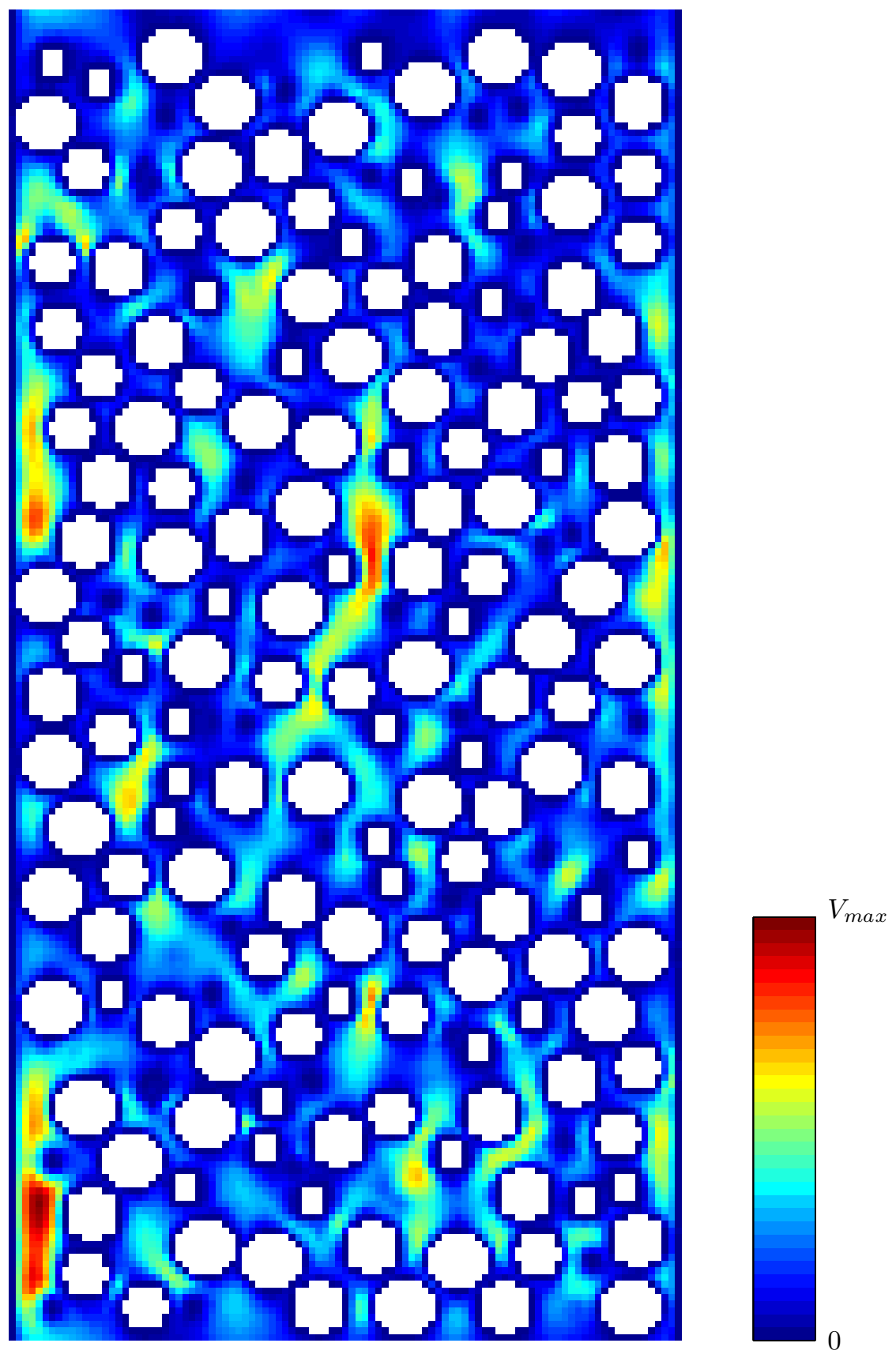

Figure 4.2: A slice through a lattice Boltzmann simulation of flow though a beadpack. The boundary conditions are periodic in the flow direction. Some artefacts of the packing algorithm (see section 4.2.2) can be seen here, such as a high porosity, the beads on the bottom sitting in the same plane and a decrease in porosity at the top. The same discretisation is used for each bead (see figure 4.4). 
the underlying grid that will be used during the LB fluid simulation. Each sphere is first random-walked along the bottom of the tube, to generate an initial position in the $X-Y$ plane. The code then random-walks the sphere upward through the beadpack, until the current sphere is above all of the preceding spheres. The sphere then random-walks downward, avoiding any overlaps with neighbouring spheres, until the sphere can no longer be moved downwards or horizontally (i.e. until it reaches a local minimum).

Once complete, the program outputs a file containing a list of the locations of the sphere-centers. As this packing algorithm tends to stack the beads unevenly, the list of sphere-centers is post-processed to remove those for which any part lies above the given height. This allows one to 'overfill' the tube, and remove the unevenly-filled upper layers to produce a more uniformly filled cylinder of the desired height. An example beadpack is shown in figure 4.3. Finally, the list of sphere centers is used to generate a set of lattice boundary conditions suitable for simulating the fluid flow via the lattice Boltzmann method. These boundary conditions are expressed as a three-dimensional array where each node indicates whether that point should be considered as fluid or solid, and in the latter case whether the node is on the surface of the solid or in the bulk. As each sphere has been constrained to lie on a grid-node, the discretisation process can use exactly the same representation for each bead, as shown in figure 4.4 .

Note that the radius of the spheres is reduced by 1 lattice spacing, $a$, before mapping them into the cubic space. This is done because the LBGK algorithm does not resolve the hydrodynamics correctly in channels that are less than 4 lattice units wide [124], and therefore reducing the bead radius helps to ensure that the flow simulation reaches a steady state. This has a significant affect on the overall porosity of the discretised beadpack, which for coarse grids can be as high as 0.7 , much larger than the $\sim 0.4$ expected for random bead packs. However, the porosity of the discrete system is calculated by counting numbers of solid and fluid lattice sites. 


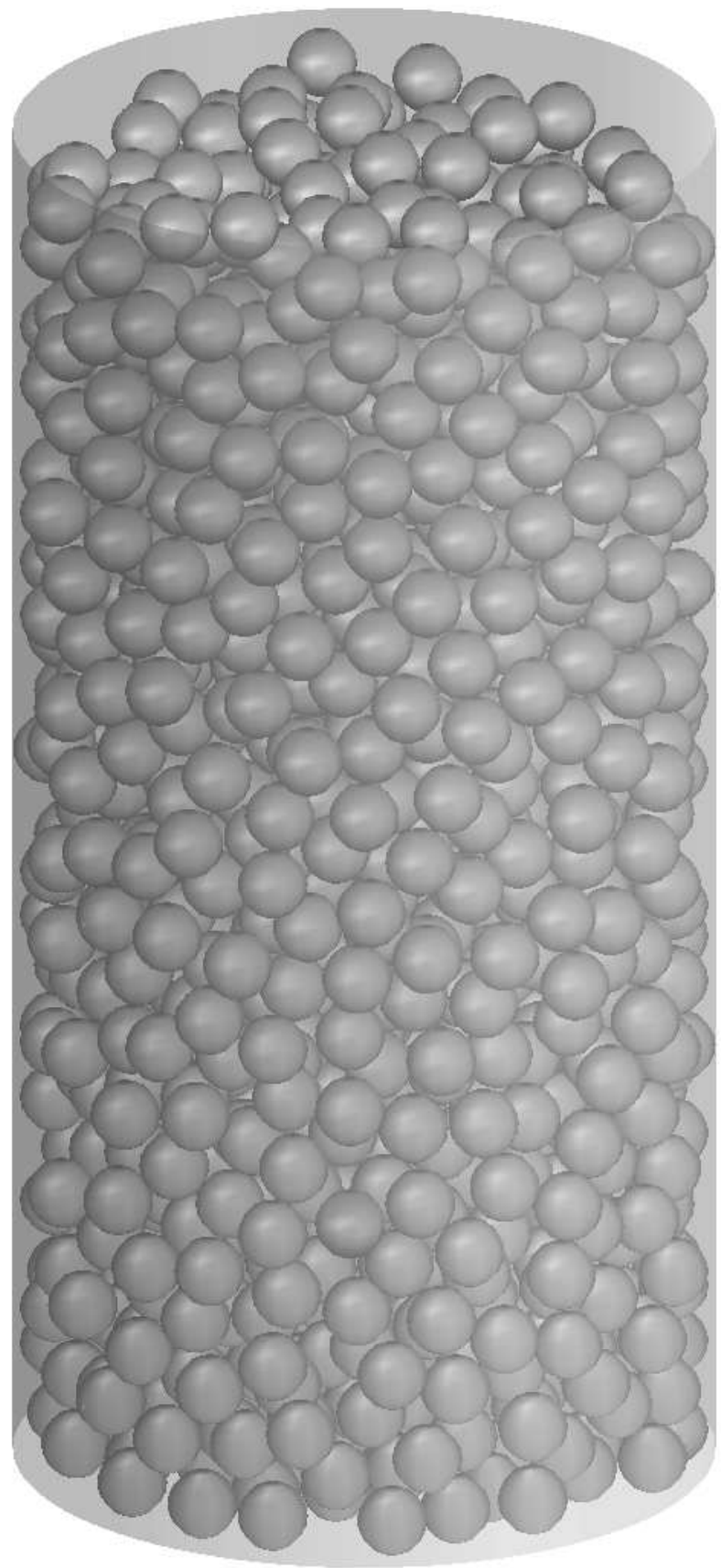

Figure 4.3: A complete beadpack, with the beads represented as spheres 


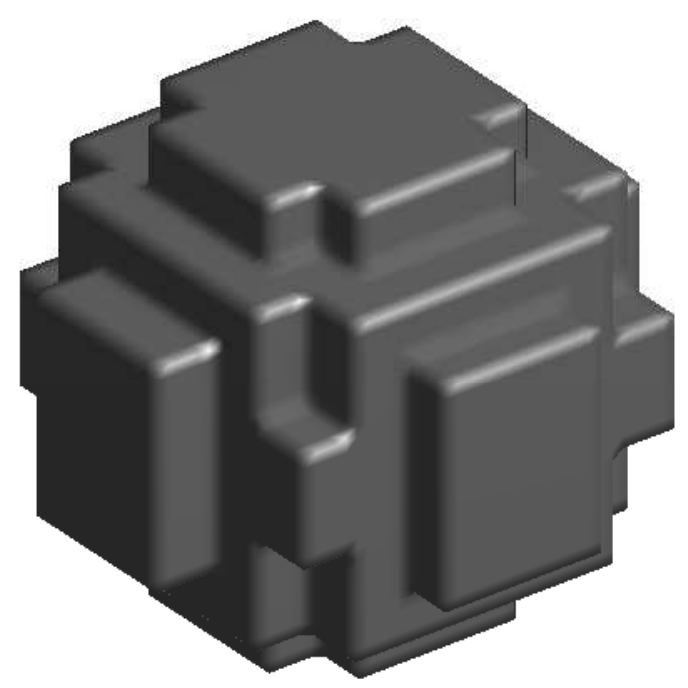

Figure 4.4: A single bead as discretised onto a lattice, the same discretisation is used for all beads.

This is likely to be something of an under-estimate on such a coarse mesh, as we would ideally use the effective hydrodynamic radius of the particles which is difficult to estimate directly. Having explored a range of discretisation algorithms, we have found this radius-reduction method to be a simple way of constructing a computable system while still producing the overall flow and dispersion behaviour characteristic of this type of system [144].

\subsubsection{Tracer Particle Simulation}

The lattice Boltzmann simulation defines a geometry and flow field on an evenly spaced grid. In contrast, the flow and diffusion of the tracer 
particles are unconstrained in space. Initially the particles are randomly distributed over the pore space. An individual lattice site defines the middle of a voxel and therefore a tracer particle can be placed up to halfway between a fluid site and a surface site. If a particle is surrounded by 8 fluid sites, the velocity is calculated with a tri-linear interpolation. If one or more of the surround sites is a surface, this is indicative of a boundary midway between the surface and the fluid site. An interpolation is then used with zero velocity at the boundary surface. This is depicted in figure 4.5 in two dimensions.
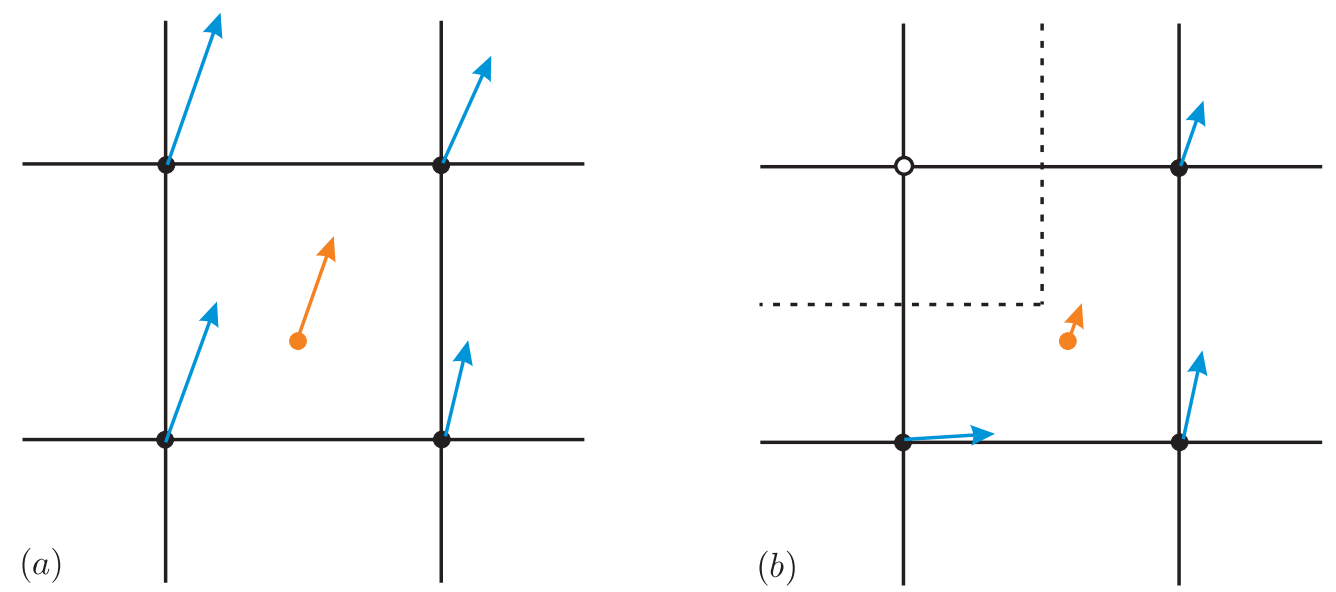

Figure 4.5: Panel (a) shows a tracer particle (orange) in the continuous space between 4 fluid lattice sites, in two dimensions. The velocity of the tracer particle is found by a linear interpolation of the lattice velocities (blue). Panel (b) shows the top left hand lattice site as a solid (open circle) which effectively gives zero velocity mid way between lattice sites (dotted line). This boundary is used for the interpolation rather than the solid lattice site.

The flow step for each tracer particle can be calculated from its velocity using Heun (mid-step) predictor corrector algorithm. For a point $\mathbf{x}_{0}$, a dummy point $\mathbf{x}_{d}$ is calculated via

$$
\mathbf{x}_{d}=\mathbf{x}_{0}+\mathbf{v}\left(\mathbf{x}_{0}\right) \Delta t
$$




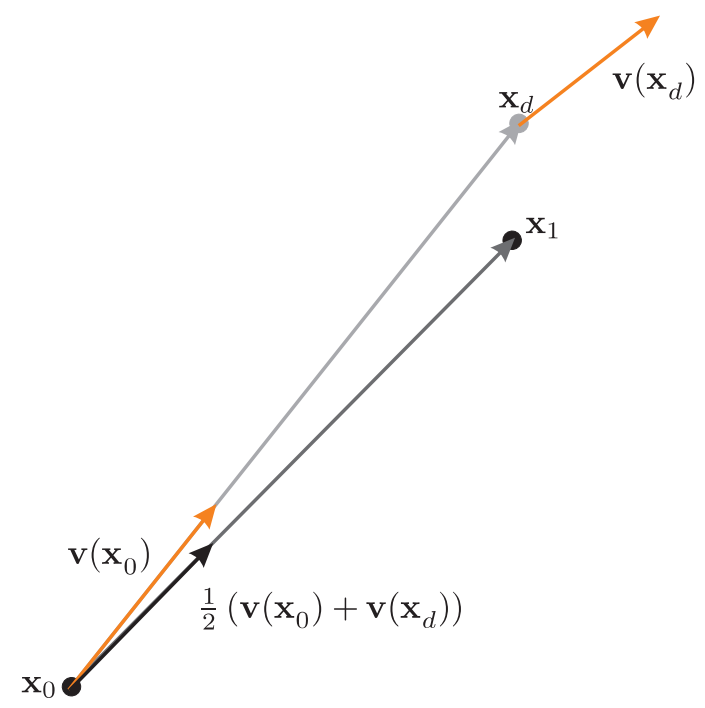

Figure 4.6: The Heun interpolation algorithm begins by finding a dummy point $\mathbf{x}_{d}$, from the velocity field at $\mathrm{x}_{0}$. The flow step from $\mathrm{x}_{0}$ is then recalculated using the average of the velocity at $\mathrm{x}_{0}$ and the velocity at $\mathrm{x}_{d}$.

the velocity $\mathbf{v}\left(\mathbf{x}_{d}\right)$ can then be interpolated. A step is then performed using the average of the initial velocity and the dummy velocity (see figure 4.6) thus the resulting step will be

$$
\mathbf{x}_{1}=\mathbf{x}_{0}+\frac{1}{2}\left(\mathbf{v}\left(\mathbf{x}_{0}\right)+\mathbf{v}\left(\mathbf{x}_{d}\right)\right) \Delta t
$$

Note equation 4.7 is a simple extrapolation technique for calculating the flow step. A tracer pathway calculated using simple extrapolation will tend to diverge under velocity gradients. This is shown in figure 4.7, also shown is the convergence of a tracer pathway calculated using equation 4.8. The Heun predictor corrector algorithm is known to be weakly second-order convergent for our system [145].

To facilitate the calculation of both the flow and diffusion, equation 4.8 is implemented as

$$
\mathbf{x}_{1}=\mathbf{x}_{0}+\frac{1}{2}\left(\mathbf{v}\left(\mathbf{x}_{0}\right)+\mathbf{v}\left(\mathbf{x}_{d}\right)\right) \Delta t+\mathbf{w}
$$




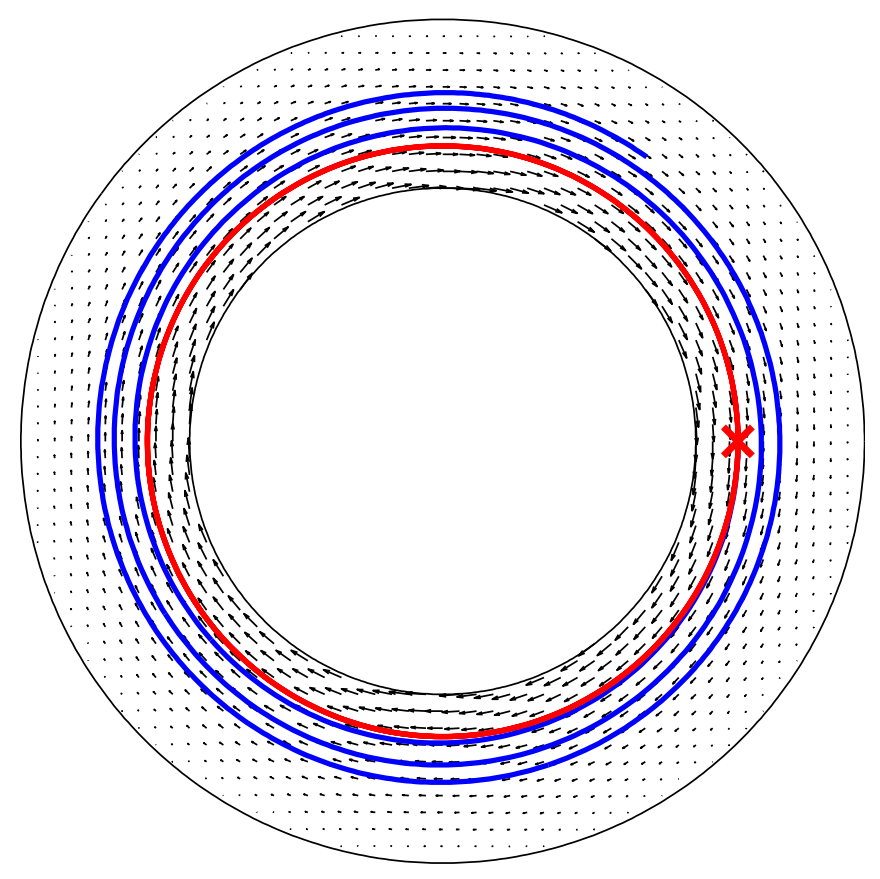

Figure 4.7: Two tracer pathways calculated for Couette flow using the Heun predictor algorithm (red) and calculated from velocity extrapolation (blue). Both stream lines start from the same position, denoted by a red cross. The radius of the outer cylinder is 1 , the resolution of the velocity grid is 0.04 , and the time increment $\Delta t$ is chosen to give the displacement step as 0.02 . The divergence of the extrapolation streamline is clear.

where $\mathbf{w}$ is a variable with a three dimensional Gaussian distribution and a variance of $2 D \Delta t$ in each dimension. The diffusion jump $\mathbf{w}$ negated the need for higher order extrapolation schemes and more sophisticated velocity interpolation methods. If a step is generated that results in the particle being place inside the solid, the step is rejected and a new step is generated. Note this is in contrast to the pure reflection used in the diffusion simulation described in section 6.2.3. 
In practice, as the simulation progresses the particles show a slight tendency to congregate at low velocity regions, resulting in a reduced average velocity. This reduction appears to be dependent on the specified Peclet number (see section 2.3.3) and while this results in a compressible flow, the effects on the VACF and hence dispersion, are small. To further minimise artifacts from this effect, a long settling time, $10 \tau_{v}$ is used. Selected tracers for an encoding time of $9.04 \tau_{v}$ are shown in figure 4.8 .

\subsubsection{Statistical Calculation of Parameters}

At predefined time intervals the position and velocity of each tracer particle is recorded. These times correspond to the application of gradient pulses in figure 5.1 and allow statistical calculation of the various quantities of interest. The recorded $z$ positions can be used to calculate the displacement of the $j$ th tracer $Z^{j}=z^{j}(\tau)-z^{j}(0)$. A propagator is generated through a histogram of the displacements. In order to calculate the nonlocal dispersion, the contribution to the VACF from each tracer particle, $u^{j}(\tau) u^{j}(0)$, is added to a bin depending on the displacement of the tracer particle.

\subsubsection{Sample Results}

Figure 4.9 shows some sample results from the simulation suite. A beadpack was constructed with 1263 beads of diameter $d_{\text {bead }}$ in a cylinder with a diameter of $10 d_{b e a d}$ and a height of $20 d_{\text {bead }}$ (see figure 4.3 ). The porosity of the discretised beadpack was 0.710, a sample slice from the lattice Boltzmann simulations being shown in figure 4.2. For the dispersion simulations the Peclet number was chosen as 1620, giving a diffusion coefficient, $D_{0}$, as $1.51 \times 10^{-3} d_{\text {bead }}^{2} / \tau_{v}$. The recorded $z$ positions of each tracer particle can be composed into a histogram of displacements, giving the propagator (figure 4.9(a)), here expressed in terms of velocity. A total of $5.4 \times 10^{6}$ tracers were used for this simulation. Significant mixing is seen only after 


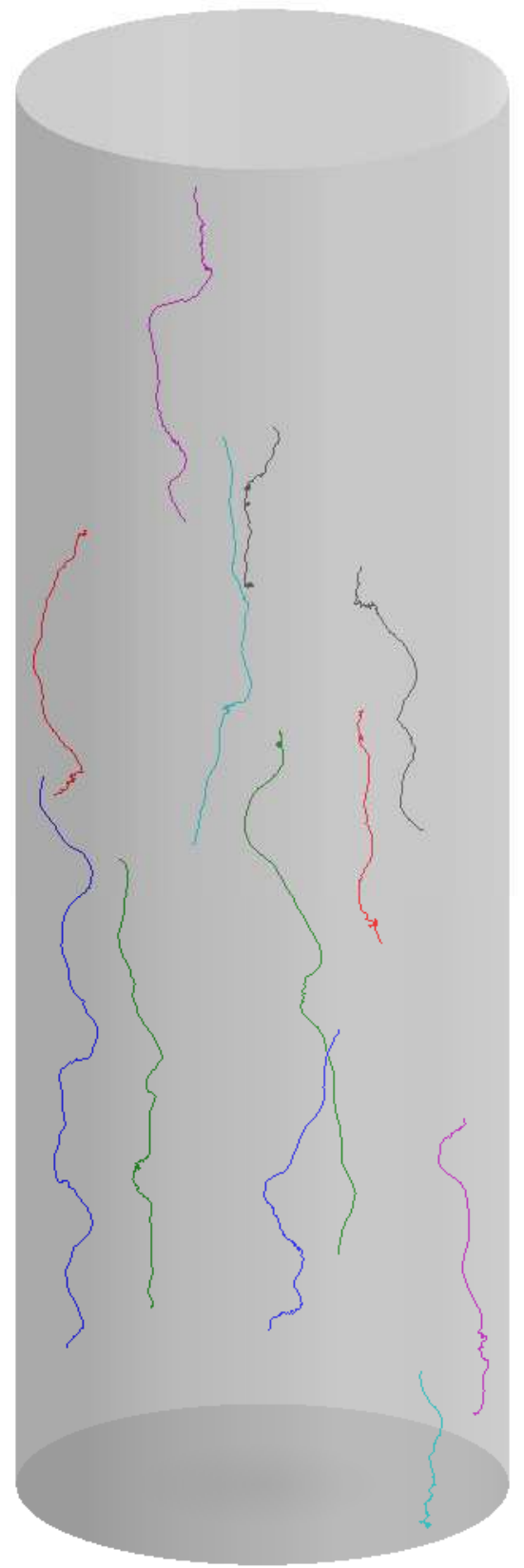

Figure 4.8: Selected tracers paths for Pe 1600. The total simulation time was $9.04 \tau_{v}$. The continuous, non-discretised cylinder is shown, but the beads are omitted. 

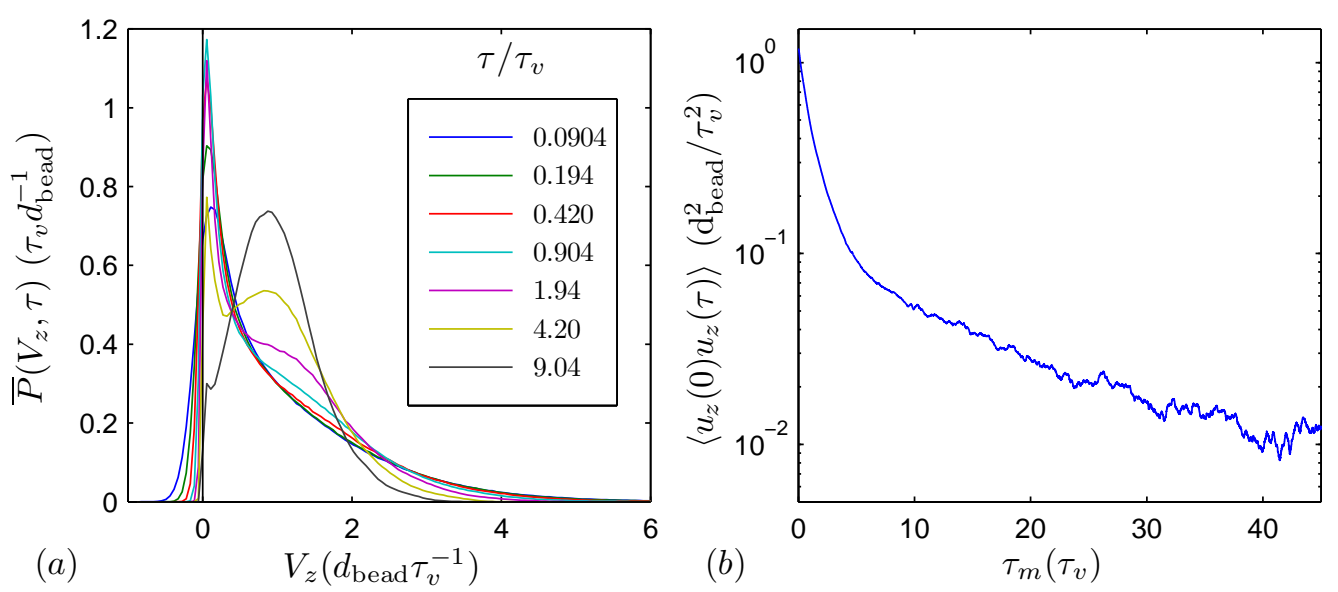

Figure 4.9: (a) The propagators in the longitudinal direction for encoding times of 0.0904 0.1940 .4200 .9041 .944 .20 and $9.04 \tau_{v}$. (b) the longitudinal velocity autocorrelation function.

an encoding time of $0.904 \tau_{v}$ and the leading peak of slow moving tracers is still dominant at an encoding time of $4.20 \tau_{v}$. The velocity autocorrelation function, for the same Peclet number but over a longer simulation time and $8 \times 10^{5}$ tracers is shown in figure 4.9 . The presence of the long tail is discussed further in [144].

\subsection{Lattice Boltzmann, Dispersion and NMR}

The use of lattice Boltzmann simulations is widespread in areas such as mass and heat transport in physics, reactor design and modelling in chemical engineering and behaviour of colloidal suspensions in bi-continuous fluids. Here we briefly review some of the work relevant to our simulations.

In [135] a hard sphere Monte-Carlo settling algorithm was used to generate random bead packs of various geometries and porosities ranging from 0.359 to 0.432 , in order to compare with published experimental re- 
sults. In some cases the bead diameter, after construction of the bead pack, was increased slightly to obtain a lower porosity. A fully parallelised (512 processors), high resolution $(512 \times 512 \times 512)$ lattice Boltzmann simulation was used to simulate the flow field.The idea of direct comparison with a high resolution NMR velocity measurement was proposed but not implemented. Comparisons were made between the simulated velocity distributions and NMR measurements of propagators. In the simulations, the experimental diffusive effects were approximated by a small amount of Gaussian broadening in the distributions.

One novel approach from Manz et al. [15] was to create the lattice directly from a binary gated magnetic resonance image (MRI). The resolution and grid size readily achieved with MRI is comparable to that which can be calculated with moderate computing power. The lattice Boltzmann simulation of the flow can then be directly compared with $2 \mathrm{D}$ velocity measurement and the agreement is generally very good. A algorithm for simulating tracer particles was also used for direct comparison with measurements of propagators and other ensemble averaged quantities [15]. The agreement, over a wide range of average displacements, was very good.

In [146] a random walk tracer particle simulation, similar to that described above, was used to fully simulate the diffusive part of the transport. This enabled study of the time evolution of dispersion. Detailed comparisons were made with published experimental NMR data. The particle tracking method for simulating dispersion was found to not support the moment propagation methods of [49]. Tracer particle simulations on a lattice Boltzmann velocity field have also been performed in [147].

Further investigation in simulation of the packing ratio (bead diameter to cylinder diameter) and its influence on the approach to asymptotic conditions was investigated in [148]. In [149] structure, radial porosity effects and dispersion were investigated with degrees of bead diameter poly dispersivity. Comparisons were made with PGSE NMR measurements of 
propagators and dispersion measurements. The dispersion in the NMR experiments was higher, but this may be due to the unexplained ordering in the experimental beadpack as seen by MRI [149].

Similar simulations have also been performed using different methods for calculating the flow field. Lebon [94] used a finite-difference scheme was used to solve Stokes' equation [150] in a simulated porous media and no tracer particle simulation was used. To compare simulated velocity distributions with experimental distributions, measurements were implemented to minimise diffusive effects. Stapf et al. [23] also used the finite difference scheme on a discetised bead pack. Tracer particle simulations were used to simulate dispersion and two-dimensional propagators. Comparisons of propagators between experiments and simulation, using finite difference and tracer particles, were also performed by [151]. As a further example of different methods for simulating the flow field, one and two phase propagators have also been calculated using a connected 3D pore network model for a porous medium [152].

Lattice Boltzmann simulation techniques have also been used for modelling two phase flow in pack bed reactors and comparing the results with NMR measurement [153]. Non-Newtonian fluids in porous media have also been modelled with lattice Botlzmann [154] and compared with NMR velocity images of flow of a shear thinning fluid in a random bead pack [155].

Recently, following a model from [156], researchers have made comparisons between simulation and experimental flow of dilute colloidal particles in porous media using NMR [157]. The flow field was generated using a lattice Boltzmann simulation, the tracer particle algorithm incorporated an exclusion mechanism due to the finite size of the colloid. A drag force as a colloid moves along a surface was also included. 


\subsection{Conclusions}

Our simulation suite has been developed to provide a completely independent dispersive system to that which we measure experimentally. The lattice Boltzmann code, while implemented to be able to executed on a parallel processing machine, is typically run on a desktop PC resulting in smaller grid sizes. This results in the porosity of the bead packs in simulation being significantly higher than that which is measured experimentally. This is not seen as a significant disadvantage since we are able to compare simulations qualitatively with experiments. These are discussed in section 7.5.

The simulation suite provides an excellent test of the post processing described in section 5.4.2. The generation and treatment of the simulated echo attenuation is described in section 5.5. On establishment of the simulation suite we now have a efficient and convenient means of exploring a range of parameters that are either outside of what can be accessed by NMR or would be tedious to perform. Some of these are discussed in section 7.5.4 and chapter 8. 


\section{Chapter 5}

\section{NMR for Nonlocal Dispersion}

\subsection{Introduction}

In this chapter we build on the PGSE-NMR techniques introduced in section 3.3 and show, in detail, the methodology used to extract the nonlocal dispersion tensor. A new pulse sequence is described with the requisite data processing techniques and practical limits of the pulse sequence will be determined. The use of the pulse sequence in relation to asymptotic dispersion will be investigated.

Actual data from flow in a bead pack is used to exemplify the processes but full discussion of the experimental set-up and interpretation of these results is left for chapter 7 . The discussion of the experimental set-up and results here will only be in the context of evaluating the experimental procedure. In order to further test our experimental procedure we have also used the simulation suite (described in chapter 4) to generate a simulated echo attenuation function. This enables a verification of the post processing techniques via comparison with statistically derived quantities.

The pulse sequence described here is also used for the experimental procedure in chapter 6 where measured data is compared to calculated quantities. The results and experimental challenges involved in the implementation of this technique are reserved for section 6.2.4. 


\subsection{The Nonlocal Dispersion Tensor}

The nonlocal dispersion tensor, $\mathrm{D}^{N L}$, introduced in section 2.4.3, is recognised as being of fundamental importance to mass transport in porous media. In PGSE-NMR it is particularly relevant because ensemble averaged techniques give access to short time and displacement scales where a nonlocal description is essential. The definition of the nonlocal dispersion tensor is given as

$$
\mathbf{D}^{N L}(\mathbf{R}, \tau)=\int \mathbf{u}_{E}(\mathbf{r}, 0) P(\mathbf{r}) P(\mathbf{r} \mid \mathbf{r}+\mathbf{R}, \tau) \mathbf{u}_{E}(\mathbf{r}+\mathbf{R}, \tau) d \mathbf{r}
$$

The compact notation of the nonlocal dispersion tensor, used henceforth, is

$$
\mathbf{D}^{N L}(\mathbf{R}, \tau)=\langle\mathbf{u}(0) \bar{P}(\mathbf{R}, \tau) \mathbf{u}(\tau)\rangle,
$$

which can be easily be interpreted as a displacement resolved velocity autocorrelation function. A PGSE technique therefore requires two velocity encodings, giving a correlation function. Simultaneously, an encoding for displacement is also necessary.

The tensor $\mathbf{D}^{N L}(\mathbf{R}, \tau)$ should also be amenable to direct measurement using PGSE NMR. One earlier such conjecture, by Ding and Candela [24], indicated the need to encode the NMR signal with information concerning the displacement propagator. However, the measurement of $\mathbf{D}^{N L}$ requires that the experiment also be sensitive to velocities separated in space and time. We here demonstrate such a method.

\subsection{NMR Implementation}

\subsubsection{The Pulse Sequence}

We begin with the pulse sequences shown in figure 5.1. Each is twodimensional in encoding gradient, and the signal superposition resulting 
from this pair enable extraction of the components of the nonlocal dispersion tensor. The first sequence shown in figure 5.1(a) is termed "compensated" since mean flow effects are nulled in the double-PGSE dimension, while that shown in 5.1(b) is uncompensated resulting in a net phase shift due to mean flow. Note that as shown, with unique phases for the RF pulses, each of these sequences contain a superposition of compensated and uncompensated phase terms arising from the flow. In order to ensure that pure compensated and uncompensated phase shifts result, an appropriate RF phase cycle is required.

Note that the encoding dimensions, $\mathbf{q}_{D}$ and $\mathbf{q}_{u}$ relate respectively to the dynamic displacement $\mathbf{R}$ and the local velocity $\mathbf{v}$. The data analysis and pulse implementation for each dimension is different. Analysis in the displacement dimension requires the displacement encoding to be in the propagator sense, so that full attenuation is achieved and the propagator information can be recovered via a fourier transform. This encoding is implemented using a Bi-Polar PGSTE [89] with each pulse having a strength $\mathrm{g}$ and a duration of $\delta$. By contrast, in order to get the necessary velocity encoding in the second dimension, the experiments must be performed and analyzed in the low- $q$ limit, as previously used in measurements of the VACF using double PGTSE experiments [16]. Each pair of gradients, with a strength of $\mathbf{g}_{\mathbf{u}}$ and duration of $\delta$ are separated by a time $\Delta_{u}$. Note that our definition of $q$ will be different for the two dimensions: in the displacement dimension $\mathbf{q}_{D}=(2 \pi)^{-1} \gamma 2 \delta$ g such that $q$ is the Fourier conjugate of displacement, and in the velocity encoding dimension $\mathbf{q}_{u}$ is the phase conjugate of velocity such that $\mathbf{q}_{u}=\gamma \delta \Delta_{u} \mathbf{g}_{u}$ where $\gamma$ is the magnetogyric ratio. The two dimensions of gradient pulses are generally implemented concurrently with the same pulse duration, $\delta$, such that when the displacement and velocity encoding directions are the same, the gradient pulses superpose.

The time $\tau$ which denotes the separation of the double PGSTE pulses represents a 'mixing-time' in the NMR sense. This time provides the tem- 


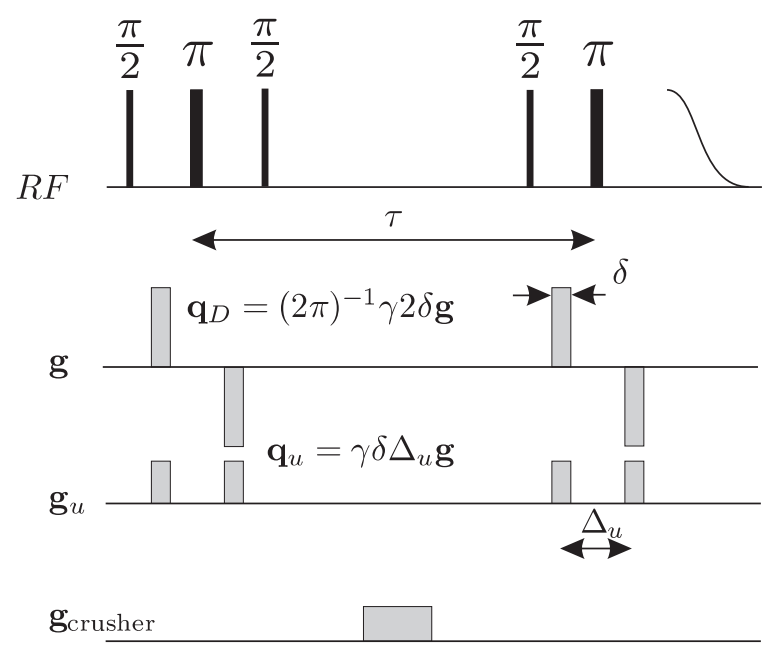

(a)

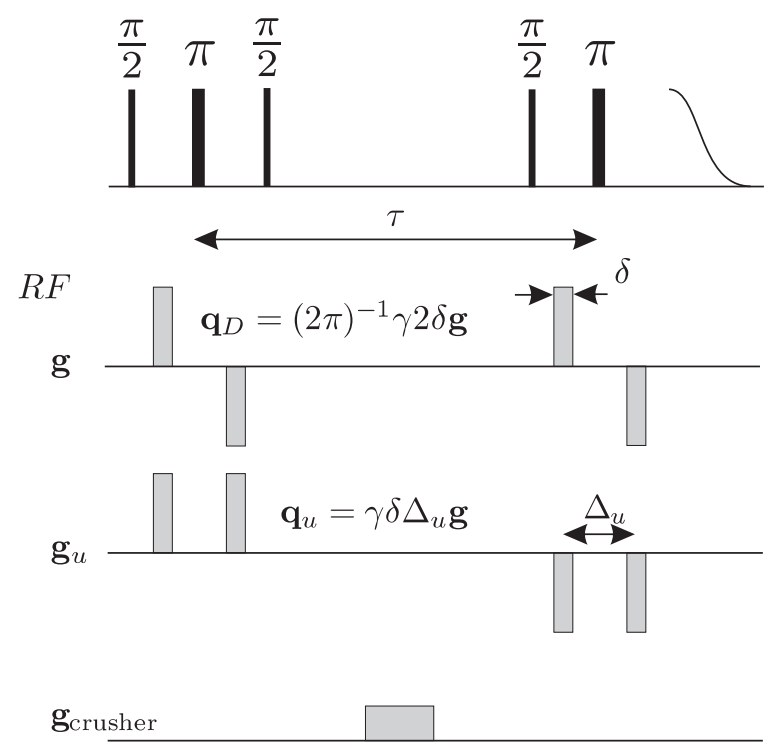

(b)

Figure 5.1: The 'compensated' (a) and 'uncompensated' (b) versions of the pulse sequence. A superposition is required when post-processing the signal.

poral dimension for fluctuations in the velocities, essentially the timescale for velocity correlation. Of course, while our pulse sequence is a double 
PGSTE with regards velocity encoding, in a displacement sense, it is set up as a single PGSTE encoding, and we will refer to $\tau$ as an 'encoding-time' in that context.

\subsubsection{The NMR Signal}

The normalised signal acquired at the final spin echo from the sequence shown in figure 5.1(a) may be written as

$$
\begin{aligned}
E\left(\mathbf{q}_{D}, \mathbf{q}_{u}\right)= & \iint \exp \left(i 2 \pi \mathbf{q}_{D} \cdot \mathbf{R}\right) \exp \left(-i \mathbf{q}_{u} \cdot\left(\mathbf{u}_{E}(\mathbf{r}, 0)+\langle\mathbf{v}\rangle\right)\right) \\
& \times P(\mathbf{r}) P(\mathbf{r} \mid \mathbf{r}+\mathbf{R}, \tau) \exp \left(i \mathbf{q}_{u} \cdot\left(\mathbf{u}_{E}(\mathbf{r}+\mathbf{R}, \tau)+\langle\mathbf{v}\rangle\right)\right) d \mathbf{R} d \mathbf{r} .
\end{aligned}
$$

Taking the inverse Fourier transform in the $\mathbf{q}_{D}$ dimension, $\mathcal{F}_{\mathbf{q}_{D}}^{-1}\{\ldots\}$, gives us an expression we call $S$, a function of $\mathbf{R}$ and $\mathbf{q}_{u}$,

$$
\begin{aligned}
S\left(\mathbf{R}, \mathbf{q}_{u}\right)=\mathcal{F}_{\mathbf{q}_{D}}^{-1}\left\{E\left(\mathbf{q}_{D}, \mathbf{q}_{u}\right)\right\}= & \int \exp \left(-i \mathbf{q}_{u} \cdot \mathbf{u}_{E}(\mathbf{r}, 0)\right) P(\mathbf{r}) P(\mathbf{r} \mid \mathbf{r}+\mathbf{R}, \tau) \\
& \times \exp \left(i \mathbf{q}_{u} \cdot \mathbf{u}_{E}(\mathbf{r}+\mathbf{R}, \tau)\right) d \mathbf{r}
\end{aligned}
$$

Note that the integral over starting position $\int \ldots d \mathbf{r}$ can be also be represented by an ensemble average notation $\langle\ldots\rangle$. The displacement information is implied through the average propagator $\bar{P}(\mathbf{R}, \tau)$ so that $S$ becomes

$$
S\left(\mathbf{R}, \mathbf{q}_{u}\right)=\left\langle\exp \left(-i \mathbf{q}_{u} \cdot \mathbf{u}_{E}(0)\right) \bar{P}(\mathbf{R}, \tau) \exp \left(i \mathbf{q}_{u} \cdot \mathbf{u}_{E}(\tau)\right)\right\rangle .
$$

Using this ensemble average notation we can write the nonlocal dispersion tensor compactly as

$$
\mathbf{D}^{N L}(\mathbf{R}, \tau)=\left\langle\mathbf{u}_{E}(0) \bar{P}(\mathbf{R}, \tau) \mathbf{u}_{E}(\tau)\right\rangle .
$$

Henceforth the subscripts denoting Eulerian velocities will be omitted and compact notation of equation in equation 5.6 employed. However the 
strict definitions are as given in equations 2.29 and 5.1.

\subsubsection{The Low- $q$ Limit}

In the analysis of our echo attenuation data, the Fourier transformation with respect to $\mathbf{q}_{D}$ represents the first step, so that subsequent analysis is performed on $S\left(\mathbf{R}, \mathbf{q}_{u}\right)$. To obtain the desired expression for $\mathbf{D}^{N L}$ we need to expand the echo attenuation, in the $q_{u}$ dimension, in the low- $q$ limit. Taking, for example, the direction of displacement encoding and velocity encoding to be both in the $Z$ direction (also chosen as the direction of the bulk velocity), we get for the first version of the pulse sequence in which the phase shift due to the bulk flow is compensated,

$$
\begin{aligned}
& S_{\text {comp }}\left(Z, q_{u}\right)= \\
& \qquad\left(1-i q_{u} u_{z}(0)-q_{u}^{2} \frac{u_{z}(0)^{2}}{2}+i O\left(q_{u}^{3}\right)+O\left(q_{u}^{4}\right)\right) \bar{P}(Z, \tau) \\
& \left.\quad \times\left(1+i q_{u} u_{z}(\tau)-q_{u}^{2} \frac{u_{z}(\tau)^{2}}{2}-i O\left(q_{u}^{3}\right)+O\left(q_{u}^{4}\right)\right)\right\rangle,
\end{aligned}
$$

where $q_{u}$ represents the magnitude of a $\mathbf{q}_{u}$ vector applied along the $z$-axis.

Expanding in $q_{u}$ we have

$$
\begin{aligned}
S_{\text {comp }}\left(Z, q_{u}\right)= & \bar{P}(Z, \tau)+i q_{u}\left\langle u_{z}(0) \bar{P}(Z, \tau)\right\rangle \\
& -\frac{q_{u}^{2}}{2}\left\langle u_{z}(0)^{2} \bar{P}(Z, \tau)\right\rangle-i q_{u}\left\langle\bar{P}(Z, \tau) u_{z}(\tau)\right\rangle \\
& -q_{u}^{2}\left\langle u_{z}(0) \bar{P}(Z, \tau) u(\tau)\right\rangle-\frac{q_{u}^{2}}{2}\left\langle\bar{P}(Z, \tau) u_{z}(\tau)^{2}\right\rangle \\
& +i O\left(q_{u}^{3}\right)+O\left(q_{u}^{4}\right) .
\end{aligned}
$$

Because the real and imaginary parts of the data are independently handled, it is necessary to account separately for the truncation errors in $q_{u}^{3}$ and $q_{u}^{4}$. The tensor elements which we seek are present as partial coefficients of $q_{u}^{2}$. Note that the velocities in the correlation term in equation 5.5 
are denoted as being separated in time by $\tau$ because of the experimental encoding for the propagator. For terms involving velocity, but without correlation at separated times, the apparent time dependence of $u_{z}$ may be dropped.

The second version of the pulse sequence is termed uncompensated. It is performed with the sign of the second velocity encoding pulse reversed as shown in figure 5.1(b), and yields

$$
\begin{aligned}
& S_{\text {uncomp }}\left(Z, q_{u}\right)=\exp \left(i 2 q_{u}\left\langle v_{z}\right\rangle\right) \quad\left(\bar{P}(Z, \tau)+i q_{u}\left\langle u_{z} \bar{P}(Z, \tau)\right\rangle\right. \\
& -\frac{q_{u}^{2}}{2}\left\langle u_{z}^{2} \bar{P}(Z, \tau)\right\rangle+i q_{u}\left\langle\bar{P}(Z, \tau) u_{z}\right\rangle \\
& +q_{u}^{2}\left\langle u_{z}(0) \bar{P}(Z, \tau) u_{z}(\tau)\right\rangle-\frac{q_{u}^{2}}{2}\left\langle\bar{P}(Z, \tau) u_{z}^{2}\right\rangle \\
& \left.+i O\left(q_{u}^{3}\right)+O\left(q_{u}^{4}\right)\right) .
\end{aligned}
$$

Because of the lack of compensation for mean flow, an extra phase factor, $\exp \left(i 2 q_{u}\left\langle v_{z}\right\rangle\right)$ is present at the beginning of the expression. This factor is easily determined by examining the data $E\left(0, q_{u}\right)$ and can be retrospectively corrected. With this correction and with a difference superposition we have

$$
\begin{aligned}
S_{\text {comp }}\left(Z, q_{u}\right)-\exp \left(-i 2 q_{u}\left\langle v_{z}\right\rangle\right) S_{\text {uncomp }}\left(Z, q_{u z}\right)= & i 2 q_{u}\left\langle\bar{P}(Z, \tau) u_{z}\right\rangle \\
& +2 q_{u}^{2}\left\langle u_{z}(0) \bar{P}(Z, \tau) u_{z}(\tau)\right\rangle \\
& +i O\left(q_{u}^{3}\right)+O\left(q_{u}^{4}\right) .
\end{aligned}
$$

Thus the elements of the nonlocal dispersion tensor may be obtained from this superposition by fitting the real part to the $q_{u}^{2}$ dependence in the low- $q$ limit. 


\subsubsection{Extracting $\mathbf{D}^{N L}$}

Other elements of the nonlocal tensor may be obtained by different choices for the directions of $\mathbf{q}_{D}$ and $\mathbf{q}_{u}$ Note that the choices of directions for the initial and final velocity encoding need not be coincident. Equation 5.9 can be generalised using the dimension subscripts $\alpha$ and $\beta$ for the initial and final velocity, and $\gamma$, for the direction of the displacement, denoted $X$ with the appropriate subscript. The nonlocal dispersion tensor, written in this subscript form is

$$
D_{\alpha \beta}^{N L}\left(X_{\gamma}, \tau\right)=\left\langle u_{\alpha}(0) \bar{P}\left(X_{\gamma}, \tau\right) u_{\beta}(\tau)\right\rangle .
$$

Omitting the truncation errors, a general form for the superposition is

$$
\begin{aligned}
\exp \left(i q_{u \alpha}\left\langle v_{\alpha}\right\rangle-i q_{u \beta}\left\langle v_{\beta}\right\rangle\right) S_{\text {comp }}\left(X_{\gamma}, q_{u \alpha, \beta}\right) & \\
\left.-\exp \left(i q_{u \alpha}\left\langle v_{\alpha}\right\rangle+i q_{u \beta}\left\langle v_{\beta}\right\rangle\right) S_{\text {uncomp }}\left(X_{\gamma}, q_{u \alpha, \beta}\right)\right\}= & i q_{u \alpha}\left\langle u_{\alpha} \bar{P}\left(X_{\gamma}, \tau\right)\right\rangle \\
& +i q_{u \beta}\left\langle\bar{P}\left(X_{\gamma}, \tau\right) u_{\beta}\right\rangle \\
& +2 q_{u \alpha} q_{u \beta}\left\langle u_{\alpha}(0) \bar{P}\left(X_{\gamma}, \tau\right) u_{\beta}(\tau)\right\rangle
\end{aligned}
$$

where $q_{u \alpha, \beta}$ means the first pair of velocity encoding gradients are along $\alpha$ and the second along $\beta$.

In cases where the velocity encoding is in a direction that has no bulk flow, no phase correction is necessary whereas, if the initial and final motion encodings are orthogonal and only one component is parallel to the direction of the main flow, $z$, the phase correction factor is $\exp \left(-i q_{u}\langle v\rangle\right)$ and appears in both $S_{\text {comp }}\left(X_{\gamma}, q_{u \alpha, z}\right)$ and $S_{u n c o m p}\left(X_{\gamma}, q_{u \alpha, z}\right)$. Despite the fact the neither pulse sequence is uncompensated or compensated, the oppositely signed $\mathbf{q}_{u}$ encodings still provide the appropriate superposition. If the phase correction is omitted, the resulting superposition will give tensors not in $u$, but in $v$.

Equation 5.11 contains terms linear in $\mathbf{q}_{u}$. In handling these, two experimental strategies are possible. In the first a further superposition using $S_{\text {comp }}\left(X_{\gamma},-q_{u \alpha, \beta}\right)$ and $S_{\text {uncomp }}\left(X_{\gamma},-q_{u \alpha, \beta}\right)$ is performed to eliminate the 
$q$ term. In the second the real and the imaginary parts of the data can be treated separately thus allowing the additional extraction of the two ensemble averages $\left\langle u_{\alpha} P\left(X_{\gamma}\right)\right\rangle$ and $\left\langle u_{\beta} P\left(X_{\gamma}\right)\right\rangle$.

There are some nonlocal components that can be measured without the superposition described in equation 5.11. If the correlation function $\left\langle u_{\alpha}(0) u_{\beta}(\tau)\right\rangle$ is known, in advance, to be equal to zero, then

$$
\int D_{\alpha \beta}^{N L}\left(X_{\gamma}, \tau\right) d X_{\gamma}=0
$$

independent of the choice of $X_{\gamma}$. A non-zero $D_{\alpha \beta}^{N L}\left(X_{\gamma}, \tau\right)$ must then be an odd function. For a transverse displacement encoding, the other terms in $q^{2}$ will be even functions, thus the data from one pulse sequence can be superposed with itself, flipped along the $X_{\gamma}$ direction thus

$$
\begin{aligned}
\exp \left(i q_{u \alpha}\left\langle v_{\alpha}\right\rangle \pm i q_{u \beta}\left\langle v_{\beta}\right\rangle\right) S\left(X_{\gamma}, q_{u \alpha, \beta}\right)- & \\
\left.\exp \left(i q_{u \alpha}\left\langle v_{\alpha}\right\rangle \pm i q_{u \beta}\left\langle v_{\beta}\right\rangle\right) S\left(-X_{\gamma}, q_{u \alpha, \beta}\right)\right\}= & i q_{u \alpha}\left\langle u_{\alpha} \bar{P}\left(X_{\gamma}, \tau\right)\right\rangle+i q_{u \beta}\left\langle\bar{P}\left(X_{\gamma}, \tau\right) u_{\beta}\right\rangle \\
& +2 q_{u \alpha} q_{u \beta}\left\langle u_{\alpha}(0) \bar{P}\left(X_{\gamma}, \tau\right) u_{\beta}(\tau)\right\rangle
\end{aligned}
$$

where $S$ results from either the 'compensated' or 'uncompensated' version of the pulse sequence. In practice the full superposition is usually used.

The three choices of directions available for the three different encodings give a total of 27 terms. There are six, non-zero, independent terms for flow in a porous medium. This is further shown in chapter 8 .

\subsection{Experimental Method}

The flow system used for these examples consisted of steady state flow though a random beadpack. A column of $500 \mu \mathrm{m}$ diameter spheres were contained in a cylinder with an internal diameter of $10 \mathrm{~mm}$. Water was pumped at a tube velocity of around $10.2 \mathrm{~mm} \mathrm{~s}^{-1}$, giving a $\tau_{v}$ of $49.0 \mathrm{~ms}$, a 
Peclet number of approximately 1500 and a Reynolds number of approximately 3.3. Full details are given in section 7.2.

It was found that a crucial part of the experimental set-up was to ensure stability of the flow.

\subsubsection{Pulse Sequence}

The use of a BP-PGSTE encoding for the displacement is not strictly necessary since the expected internal gradients for our bead pack are small. However, the low attenuation due the motion encoding pulses was more reliable when the pulses were separated by a $\pi$ pulse and so the displacement encoding pulses were similarly implemented.

Typically in a BP-PGSTE experiment, unwanted phase coherences due to the $\pi$ pulses can be minimised by using small crusher gradients either side of the RF pulse. These crusher gradients would normally be in a direction orthogonal to the main encoding. It is usually assumed that any attenuation caused by these gradients are small compared to the effects being measured. However in this case we do not have this freedom as it is precisely the small effects we are expecting to measure with the low$q$ motion encoding pulse. To this end we need a comprehensive 32-step phase cycle to minimise the unwanted coherences, see section 3.3.4 and figure 3.16. The complete phase cycle is given in table 5.1.

Note that the phase cycle performs two separate tasks. First, it removes baseline artifacts and the unwanted FIDs generate by all RF pulses other that the first $\frac{\pi}{2}$ excitation pulse. Second, it ensures that the appropriate superposition of orthogonal transverse plane magnetisation components are stored, so as to produce the desired pure phase shift term after recall following the storage period.

The signal averaging that accompanies the 32 steps in the phase cycle is advantageous when fitting in the low- $q$ dimension, and gives the opportunity for improved displacement resolution in the propagator. For some 


\begin{tabular}{|c|rrrrrrrr|}
\hline Excitation & xxxx & xxxx & xxxx & $x x x x$ & $-x-x-x-x$ & $-x-x-x-x$ & $-x-x-x-x$ & $-x-x-x-x$ \\
$\pi$ & $y y y y$ & $-y-y-y-y$ & $y y y y$ & $-y-y-y-y$ & $y y y y$ & $-y-y-y-y$ & $y y y y$ & $-y-y-y-y$ \\
Storage & $x-x y-y$ & $x-x y-y$ & $x-x y-y$ & $x-x y-y$ & $-x x-y y$ & $-x x-y y$ & $-x x-y y$ & $-x x-y y$ \\
Recall & $x-x y-y$ & $x-x y-y$ & $-x x-y y$ & $-x x-y y$ & $x-x y-y$ & $x-x y-y$ & $-x x-y y$ & $-x x-y y$ \\
$\pi$ & $y-y-x x$ & $y-y-x x$ & $y-y-x x$ & $y-y-x x$ & $y-y-x x$ & $y-y-x x$ & $y-y-x x$ & $y-y-x x$ \\
\hline Receiver & $x x-x-x$ & $x x-x-x$ & $-x-x x x$ & $-x-x x x$ & $-x-x x x$ & $-x-x x x$ & $x x-x-x$ & $x x-x-x$ \\
\hline
\end{tabular}

Table 5.1: The 32-step phase cycle for the pulse sequence depicted in figure 5.1. This phase cycle minimises unwanted coherences without the need for crusher gradients around the $\pi$ pulses and also ensures pure phase-encoded terms after recall following the storage period.

encoding times there is still a small amount of unwanted signal when $q_{u}=q_{D}=0$. This results in an offset at $q_{u}=0$ in $S\left(X_{\gamma}, q_{u}\right)$. The low- $q$ fit algorithm can easily ignore this point.

\section{Displacement Encoding}

The acquisition in the displacement dimension is performed using a grained approach [114], typically in three stages. This enabled sharp resolution of any stagnant or slow moving peak while still capturing the faster moving particles. If the encoding was in the longitudinal direction the displacement encoding was performed using 22 fine, 14 medium and 26 coarse increments between equal positive and negative gradients giving a total of 63 gradient values. The step ratios were 1:2:8 giving an interpolated data set with 259 values, the first 258 were used for the discrete Fourier Transform. Figure 5.2 shows the measured and interpolated $q_{D}$ data for an experiment with displacement encoding direction, $Z$, parallel to the main flow. With the exception of figures 5.8 and 5.10 all the examples shown here will be from the same pair of experiments, that which is needed for extracting $D_{z z}^{N L}(Z, \tau)$ for $\tau=46.3 \mathrm{~ms}$. For transverse encoding only a two stage graining was required 14 medium and 26 coarse increments giving a total of 41 steps. The step ratios were 1:4 giving an interpolated data set 
with 119 values. Figures 5.3(a) and (b) show that all the fine structure of the echo attenuation is resolved, this is also true when the echo attenuation is expanded into the $q_{u}$ dimension.

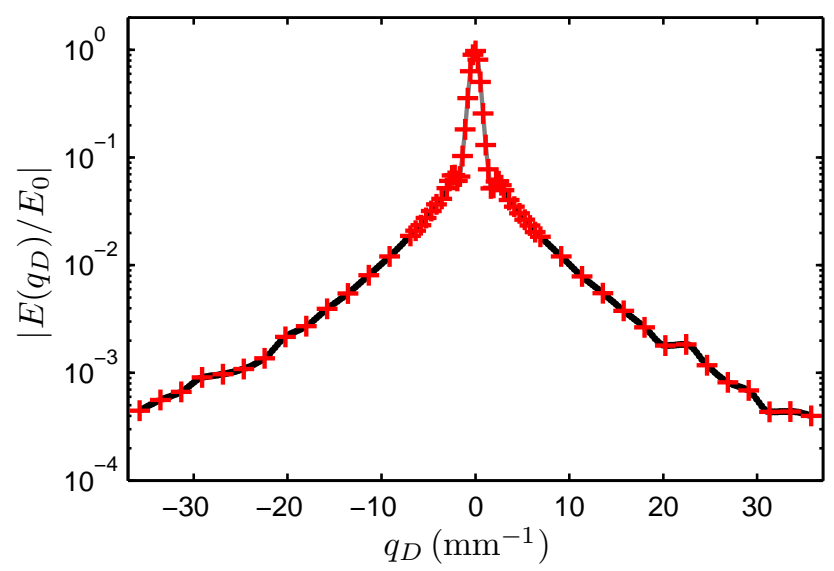

Figure 5.2: The magnitude of the echo attenuation for a longitudinal displacement encoding when $q_{u}=0$. In this case $\tau=46.3 \mathrm{~ms}$. The red crosses show measured data and the black dots show the interpolated data. The signal is fully attenuated at maximum $q$ giving a signal to noise of around 1000 .

The interpolation was performed in logarithmic space for the magnitude of the echo attenuation and a linear interpolation for the phase of the echo attenuation. These were then combined to give the conventional real and imaginary parts of the echo attenuation. No zero-filling, symmetrising or filtering was required in this dimension.

\section{Motion Encoding}

The motion encoding is performed using a pair of gradients around a $\pi$ pulse separated by $\Delta_{u}$. To increase the reliability of the gradient pairs, 1-3 'warm-up' pulse-pairs were used (see section 3.5), both before the main excitation pulse and during the storage period. For short mixing times this was not practical. For measurement of components where motion and 

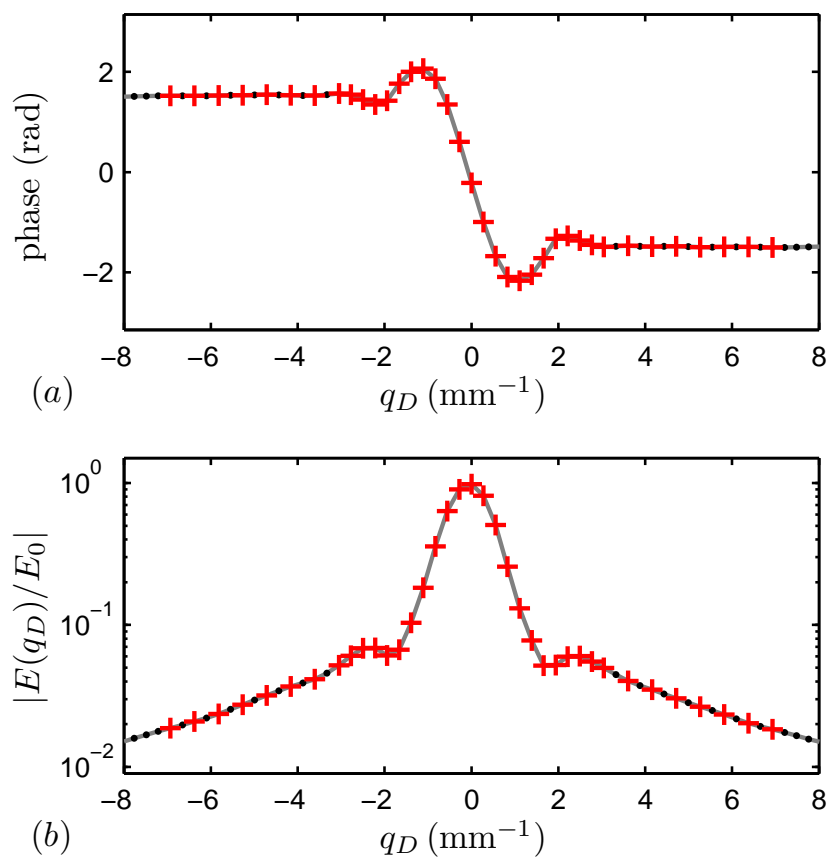

Figure 5.3: The measured and interpolated data around $q_{D}=0$ for the encoding described in figure 5.2. The red crosses show measured data and the black dots show the interpolated data. The gray line acts as a guide for the eye. The fine structure is resolved both in the phase and the magnitude.

displacement were in the same direction, the superposition of the gradient pulses were used in the 'warm-up' train.

Omitting the 'warm-up' pulses has little effect on the magnitude of the echo attenuation but it does introduce an artifact of a $q_{u}$ dependent phase shift. At first this is not seen as a problem since the $q_{u}$ dependent phase shift is compensated as is the 'real' $q_{u}$ dependent phase shift due to the bulk velocity. However when tensors in $v$ are to be examined this artifact needs to be minimised, either by using the 'warm-up' pulses or by comparing the phase shift to that measured with no flow.

Equation 5.9 suggests the $q^{2}$ fit should be performed after the superposition, however in practice the fitting is done beforehand. Treating the 
compensated and uncompensated experiments separately in this way enables independent choice of maximum motion encoding gradients. Typically 21-33 $q_{u}$ steps are used to give reliable low- $q$ fits. The total time for the pair of experiments necessary for one measurement is around 8 hours for transverse encodings and 12 hours for longitudinal.

\subsubsection{Post Processing}

The phase correction factor in equation 5.8, is found from examining the phase shift of the echo attenuation $E\left(q_{D}, q_{u}\right)$ at $q_{D}=0$. The linear term from a cubic fit is used for evaluation. The phase corrected $S\left(X_{\gamma}, q_{u}\right)$ from the uncompensated experiment will be denoted by $S_{\text {uncomp }}^{c}\left(X_{\gamma}, q_{u}\right)$.

The superposition to find $D^{N L}$ relies on the validity of the low- $q$ approximation. Previous use of this technique has been to investigate the VACF [16] and typically this low- $q$ approximation has been held to be true if the attenuation is $0.7 E_{0}$, points outside of this range were ignored and a linear fit in $q^{2}$ was made to the remainder of the data. Tests using a fit to more attenuation showed similar characteristics [16].

In nonlocal dispersion experiments, however, once each data set is Fourier transformed giving $S$, the variations in $S\left(X_{\gamma}, q_{u}\right)$ for each displacement pixel can be quite extreme. Figure 5.4 shows examples of $S_{\text {comp }}\left(Z, q_{u}\right)$ and $S_{\text {uncomp }}^{c}\left(Z, q_{u}\right)$. To provide a reliable estimate for the $q_{u}^{2}$ coefficient we need an algorithm that is relatively independent of the resulting attenuation.

For each displacement pixel of $S\left(Z, q_{u}\right)$ the low- $q$ fit was performed by first fitting an even polynomial to the magnitude and then an odd polynomial to the phase, from these the coefficients of $q^{0} q^{1}$ and $q^{2}$ from expressions 5.7 and 5.8 can reliably be estimated.

To enable the algorithm to be relatively insensitive to attenuation but also stable, a multi-stage process was used. Beginning with $N=8$, a Nth order even polynomial was fitted to the magnitude. The fit was rejected if 

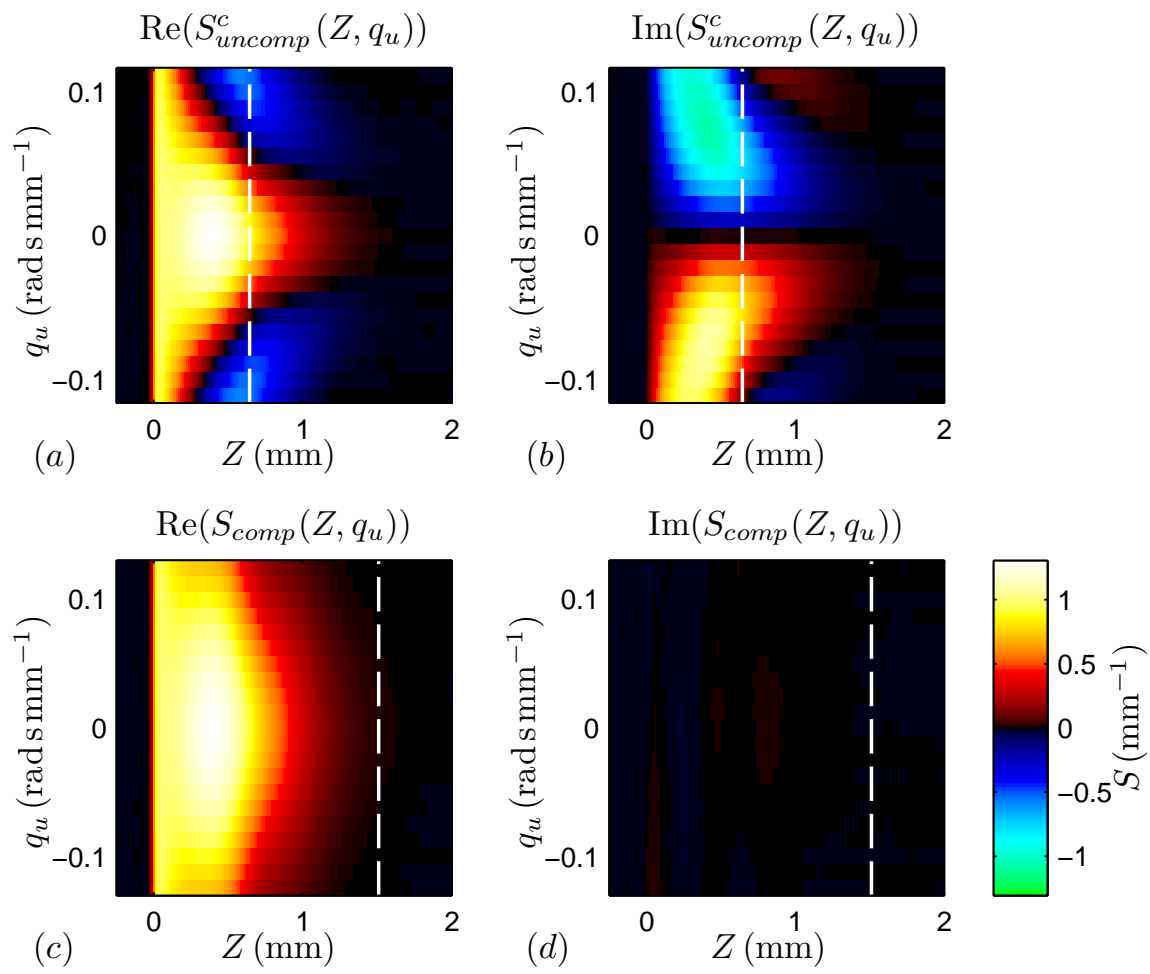

Figure 5.4: The real (a) and imaginary (b) parts of $S_{\text {uncomp }}^{c}$ and the real (c) and imaginary (d) parts of $S_{\text {comp }}$. These are the two data sets necessary to give $D_{z z}^{N L}(Z, \tau)$. In this case $\tau=46.3 \mathrm{~ms}$. The dotted white lines in panels (a) and (b) show the displacement pixels chosen as an example of the $q^{2}$ fitting in figure 5.5 and the lines in panels (c) and (d) show the displacement pixel used in figure 5.6.

the standard error in the Nth order term was greater that the magnitude of the Nth order term. A (N-2)th order fit was then tested under the same criteria until a fit to the magnitude is found. This algorithm will typically use a 6th order fit for the $q_{u}$ data with large attenuations $\left(\sim 0.2 S\left(X_{\gamma}, q_{u}=0\right)\right)$, and usually will not false fit a higher order polynomial to noise. Once the magnitude fit has been found, this is used to estimate the $q$ value, $q_{a}$ that gives an attenuation of $0.7 E_{0}$, allowing the definition of a weight function 


$$
w t=\frac{1}{\left(1+\exp \left(-\frac{A}{q_{a}}\left(q+q_{a}\right)\right)\right)\left(1+\exp \left(\frac{A}{q_{a}}\left(q-q_{a}\right)\right)\right)},
$$

which is essentially a broadened hat function where the factor $A$, typically 10 , determines the width of the broadening. The same polynomial fit is then performed with the weight function, thus reducing the truncation error of the fit and determining the points that we consider to fulfill the low- $q$ approximation. If the point $q_{a}$ can not be estimated, no weight function is used. This is typically the case for low attenuation in the $q_{u}$ direction of $S\left(X, q_{u}\right)$ and would usually result in a low order polynomial fit.

The $q_{u}$ dependent phase is fitted in a similar a fashion, using the weight function determined above and beginning with a fifth order odd polynomial until an acceptable fit is found. This phase shift is significant in the uncompensated experiment and is zero within experimental artifacts for the compensated experiment. Nevertheless the same algorithm is used to perform a fit, usually resulting in a linear fit with a small $q_{u}$ dependent term. With the two polynomial fits, the curvature of the real data around $q_{u}=0$ can be determined, with an estimate of the error.

Figures 5.5 and 5.6 show examples of the fitting algorithm for the uncompensated and compensated experiments respectively. The uncompensated example is for a displacement near the average where signal to noise is excellent. The $q_{u}$ dependent phase shift can clearly be seen in panels (c) and (d). The complex polynomial fit to the data in panel (d) allows the coefficients of $q$ and $q^{2}$ to be determined. In this case the fit to the phase was a 5 th order odd polynomial and to the magnitude was a fourth order even polynomial. The compensated example in figure 5.6 is for a large displacement, such that the signal to noise is poor. In this example the proportion of particles moving this distance is $0.02 \%$. The weight function largely ignores the points at large $q_{u}$ but the fit in panel (d) still shows good agreement around $q_{u}=0$. The magnitude was fitted with a 6 th order polynomial and the phase was fitted with a linear polynomial. 

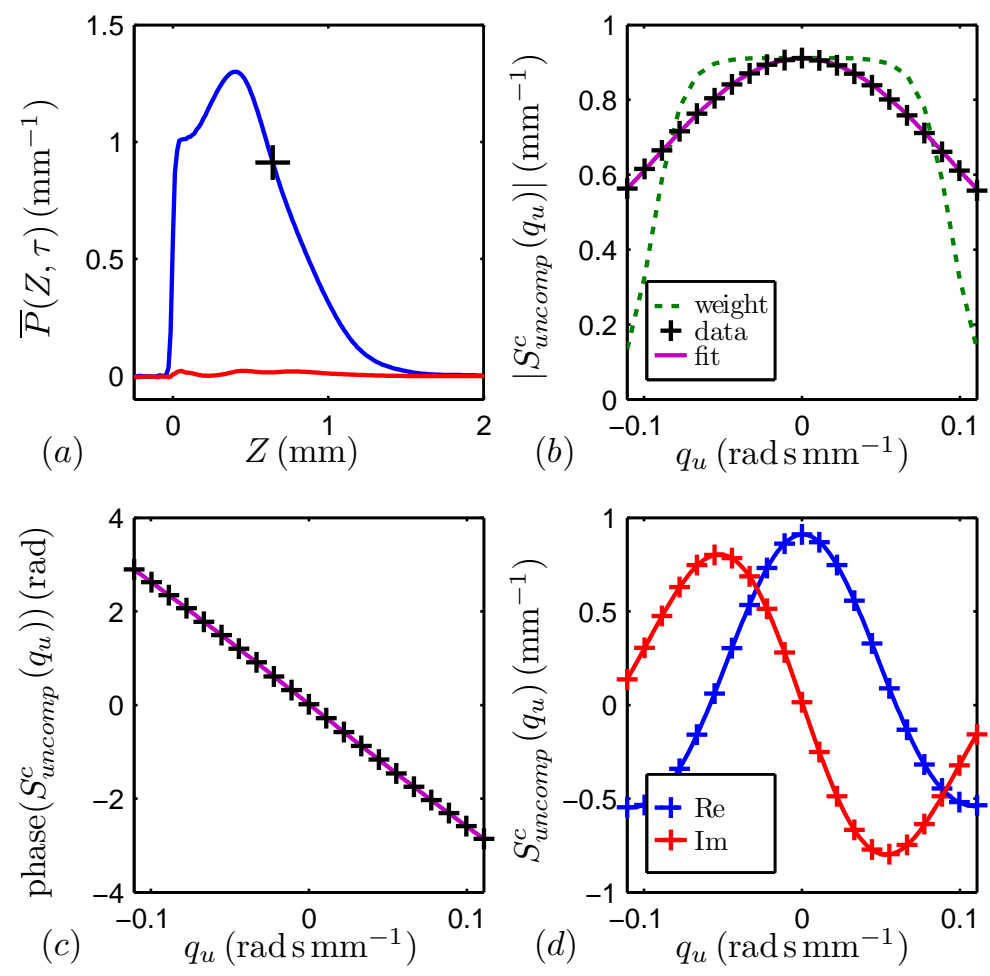

Figure 5.5: The low- $q$ fitting to the phase corrected uncompensated encoding for $D_{z z}^{N L}(Z, \tau), \tau=46.3 \mathrm{~ms}$. The full data set is shown in figure $5.4(\mathrm{a})$ and (b). The magnitude of the $q_{u}$ attenuation showing the data shown in panel (b), at the displacement pixel marked by a black cross on the propagator in panel (a). Panel (b) also shows the weight function (dotted green line) used to perform the fit, shown in magenta, of the magnitude and phase, panels (b) and (c) respectively. Panel (d) shows the complete fit to the $q_{u}$ data from which the $q_{u}^{2}$ coefficient can be estimated.

Once the fitting has been performed for each displacement pixel the superposition can be performed. Figure 5.7 continues with our example of $D_{z z}^{N L}(Z, \tau)$. In this case the differences between the two encodings is relatively large, thus giving small error bars. The need for noise insensitive, robust algorithm is highlighted in other cases, such as in the superposition 

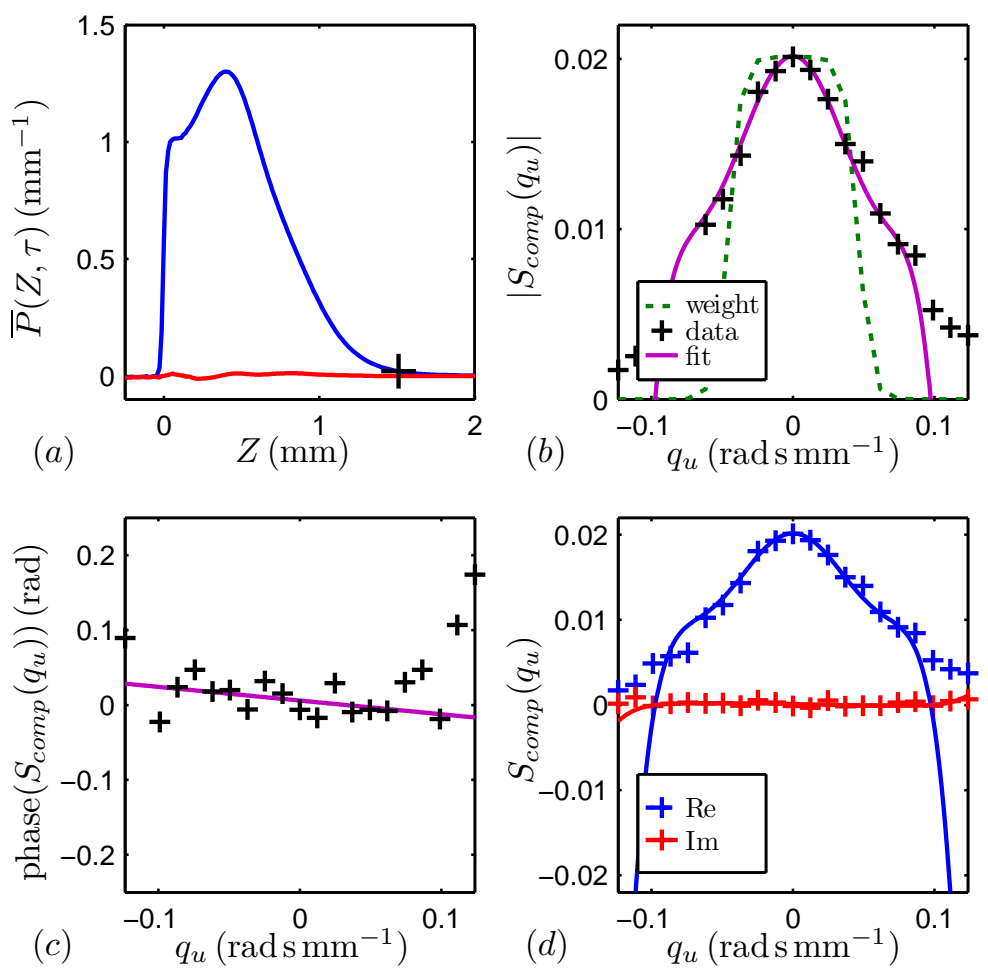

Figure 5.6: The low- $q$ fitting to the compensated encoding for $D_{z z}^{N L}(Z, \tau)$, $\tau=46.3 \mathrm{~ms}$. The full data set is shown in figure $5.4(\mathrm{c})$ and (d). The magnitude of the $q_{u}$ attenuation showing the data shown in panel (b), at the displacement pixel marked by a black cross on the propagator in panel (a). In this example the displacement is large giving poor signal to noise. Panel (b) also shows the weight function (dotted green line) used to perform the fit, shown in magenta, of the magnitude and phase, panels (b) and (c) respectively. Panel (d) shows the complete fit to the $q_{u}$ data from which the $q_{u}^{2}$ coefficient can be estimated. Clearly the weight function prevents an accurate fit to the complete data but still provides a reliable fit to the data around $q_{u}=0$.

required for the encoding describing transverse displacement and velocity correlations, $D_{x x}^{N L}(X, \tau)$ for $\tau=\tau_{v}$. Figure 5.8 shows a very small difference between the fitted data sets.

A superposition can also be made to extract various other tensors. These 

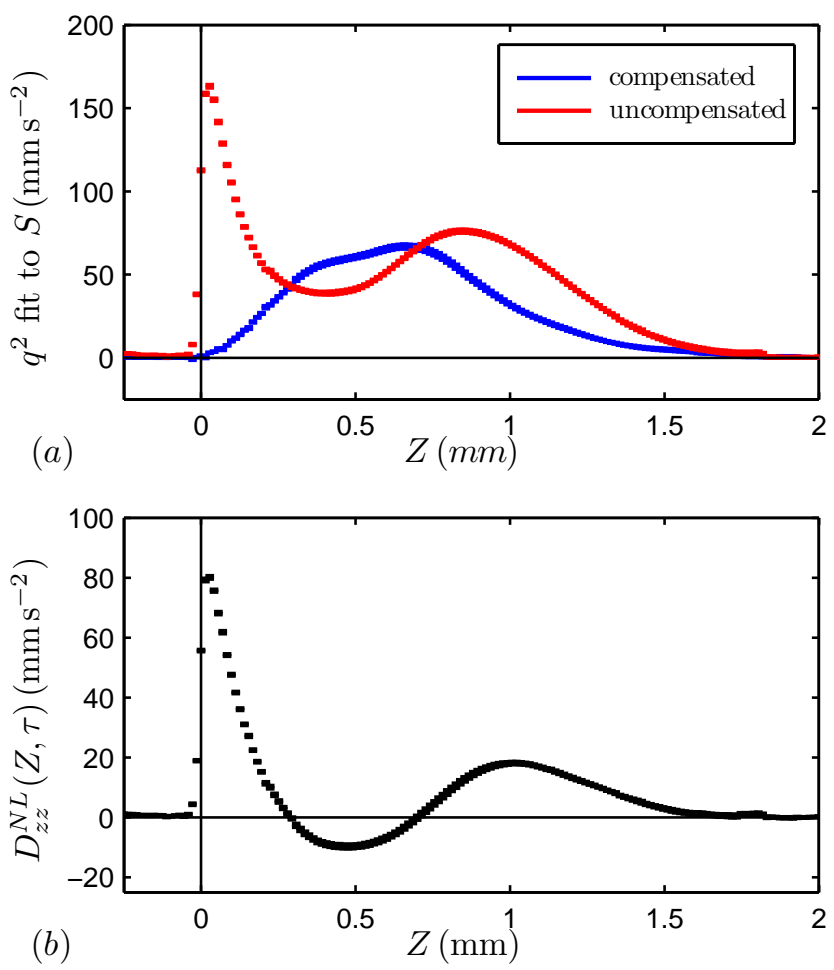

Figure 5.7: The resulting fits from $S_{\text {uncomp }}$ and $S_{\text {comp }}^{c}$, panel (a), superposed to give $D_{z z}^{N L}(Z, \tau)$, panel (b). In this instance the difference between the uncompensated and compensated fit is large, giving small error bars.

terms do not contain any information correlating velocities in space and time, but do give structure and temporal information to the first and second moments $\left\langle u_{z}\right\rangle$ and $\left\langle u_{z}^{2}\right\rangle$. The phase correction can also be omitted, giving tensors in $v_{z}$ rather than $u_{z}$. The tensors available from the experiment performed to extract $D_{z z}^{N L}(Z, \tau)$ are shown in figure 5.9, and summarised in table 5.2. 

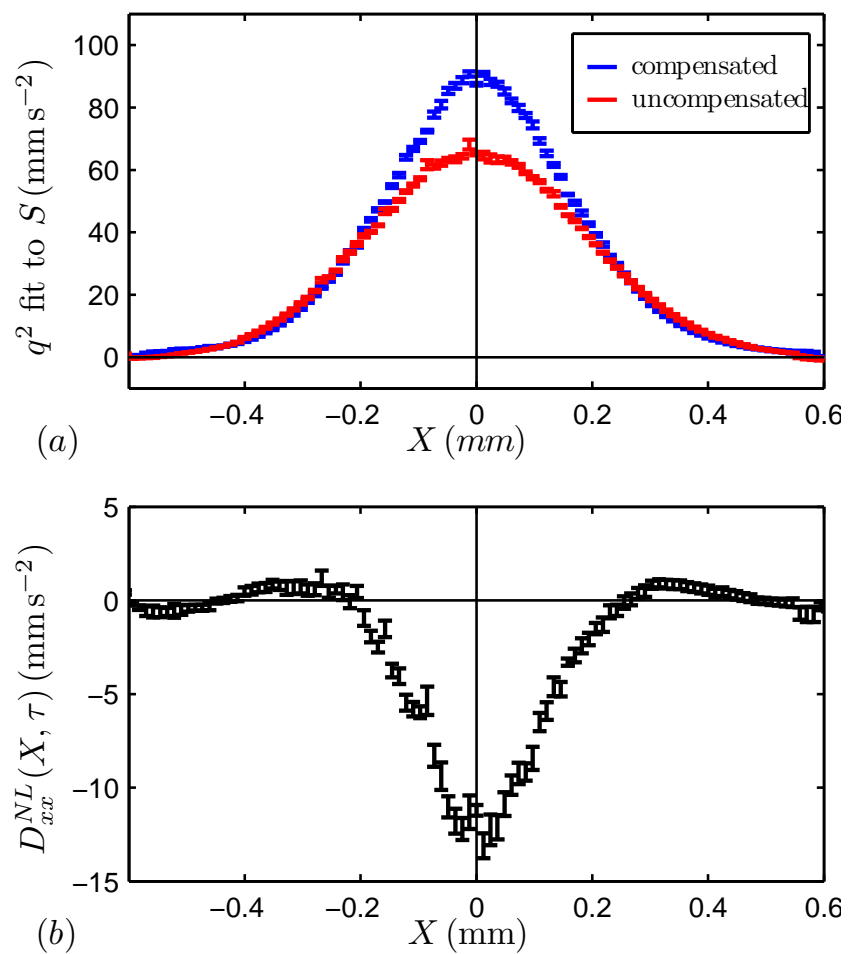

Figure 5.8: The encoding for the fully transverse term $D_{x x}^{N L}(X, \tau)$ at $46.3 \mathrm{~ms}$ shows the small difference between the fits to the $q^{2}$ data (Panel (a)). This results in significant error bars in the nonlocal term (Panel (b)).

\subsubsection{General Experimental Considerations}

The sensitive nature of the low- $q$ fitting and the superposition demand a good signal-to-noise ratio and the absence of artifacts. For most PGSENMR investigations of dispersion signal-to-noise is more than adequate due to the ensemble averaging nature of the experiment.

In order to obtain data free of artifacts it is crucial to have stable flow. In any given experiment the initial velocity encoding is typically over a period of 2-3 ms, the final velocity encoding is then $10 \mathrm{~s}$ to $100 \mathrm{~s}$ of milliseconds later. Overall a pair of experiments for one measurement would be on the order of 10 hours, thus requiring flow stability over a wide range of 


\begin{tabular}{|l|c|}
\hline Description & Quantity \\
\hline Propagator & $\left\langle\bar{P}\left(X_{\gamma}, \tau\right)\right\rangle$ \\
\hline Nonlocal dispersion tensor & $D_{\alpha \beta}^{N L}\left(X_{\gamma}, \tau\right)$ \\
\hline Nonlocal dispersion in $v$ & $D_{\alpha \beta}^{N L v}\left(X_{\gamma}, \tau\right)$ \\
\hline First moment of velocity & $\left\langle u_{\alpha} \bar{P}\left(X_{\gamma}, \tau\right)\right\rangle,\left\langle\bar{P}\left(X_{\gamma}, \tau\right) u_{\beta}\right\rangle$ \\
\hline First moment in $v$ & $\left\langle v_{\alpha} \bar{P}\left(X_{\gamma}, \tau\right)\right\rangle,\left\langle\bar{P}\left(X_{\gamma}, \tau\right) v_{\beta}\right\rangle$ \\
\hline Second moment of velocity & $1 / 2\left(\left\langle u_{\alpha}^{2} \bar{P}\left(X_{\gamma}, \tau\right)\right\rangle+\left\langle\bar{P}\left(X_{\gamma}, \tau\right) u_{\beta}^{2}\right\rangle\right)$ \\
\hline Second moment in $v$ & $1 / 2\left(\left\langle v_{\alpha}^{2} \bar{P}\left(X_{\gamma}, \tau\right)\right\rangle+\left\langle\bar{P}\left(X_{\gamma}, \tau\right) v_{\beta}^{2}\right\rangle\right)$ \\
\hline
\end{tabular}

Table 5.2: A table showing all of the displacement resolved quantities available from a pair of experiments with the initial motion encoding in the direction $\alpha$, final motion encoding in the direction $\beta$ and displacement encoding in the direction $\gamma$. Not all of these quantities will be non-zero. The measured quantities for a fully longitudinal encoding are shown in figure 5.9 panels (c) to (i).

time scales. The acquired data can be checked for self consistency by ensuring the agreement of the propagators given by $S_{\text {uncomp }}^{c}\left(X, q_{u}=0\right)$ and $S_{\text {comp }}\left(X, q_{u}=0\right)$. A further, and more demanding test, is to ensure that $E\left(0, q_{u}\right)=E^{*}\left(0,-q_{u}\right)$ for both experiments. Even small departures from either of these conditions, particularly the latter, will result in data that is not suitable for low- $q$ analysis.

\subsection{Echo Attenuation Simulation}

In order to test our experimental methodology and the associated data analysis protocols, we have carried out direct numerical calculations of the relevant dispersion tensors using a Lattice-Boltzmann/Monte Carlo simulation, and then used the simulated tracer displacements to generate echo signals appropriate to the compensated and uncompensated double PGSTE NMR experiments. We then process these simulated NMR data using the methods outlined above and compare the results with the directly 

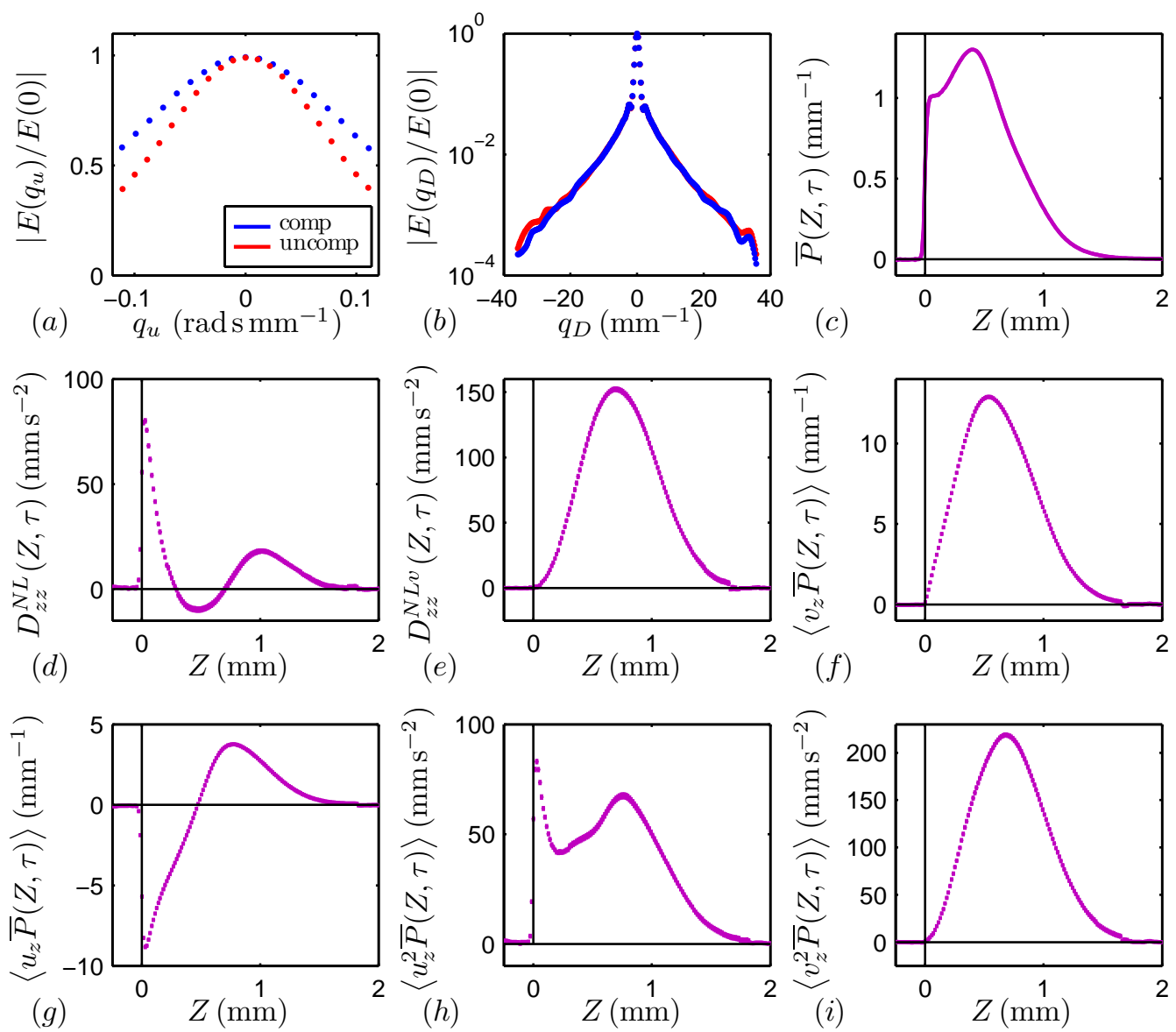

Figure 5.9: A summary of the additional data from the two experiments necessary for $D_{z z}^{N L}(Z, \tau)$. Panel (a) shows the magnitude of the attenuation from the compensated and uncompensated double PGSTE, the difference in the two is indicative of a positive velocity autocorrelation function. Panel (b) shows the magnitude of the echo attenuation for displacement encoding for each experiment. Panel (c) shows the two identical propagators. Panel (d) gives $D_{z z}^{N L}(Z, \tau)$, for $\tau=46.3 \mathrm{~ms}$. Panel (e) shows the nonlocal dispersion tensor in $v$ rather than $u$. The further tensors which are able to be extracted are shown in panels (f) and (g) for the first moment resolved by displacement, and panels (h) and (i) give the second moment resolved by displacement. 
computed tensor elements.

The simulation consists of three components, the bead pack generator, the lattice Boltzmann (LB) flow generator, and the virtual tracer particle simulation component. These components are combined in order to build a suitable beadpack, model the flow through it, and then model the motions of virtual tracer particles through that flow field. The lattice Boltzmann code provides a way of statistically measuring the tensors we are interested in. The results between the simulated nonlocal dispersion in a algorithm-generated beadpack compare well with experimental measurements of a random bead pack [158] although large differences in porosities between our simulated beadpacks $(\sim 0.7)$ and experimental beadpacks $(\sim 0.4)$ preclude exact agreement. The simulated propagator of figure 5.10(c) clearly shows more slower moving particles than the experimental propagator of figure 5.9(c). These effects, and are discussed further in chapter 7 .

Statistical calculation of the nonlocal dispersion tensor is performed by calculating the contribution to the velocity autocorrelation function from each tracer particle and putting this value in a bin, depending on how far the tracer particle has moved. The measurement of initial and final velocity of each tracer particle can be done two ways. First, using the particle's instantaneous velocity as given by its position in the local velocity field and second, by using an average velocity over some finite encoding time. The latter is similar to the experimental method where the velocity encoding is performed using a duration of $\Delta_{u}$. In figure 5.10, panels (d) to (i) the tensors calculated using the instantaneous velocity are labelled as 'Statistical $(v)^{\prime}$ and the tensors calculated using a finite encoding time are labelled as 'Statistical $\left(\Delta_{u}\right)$ '.

Generating the expected echo attenuation function is easily performed using the tracers in the simulation. Each particle begins the simulation with zero phase, a $\mathbf{q}$ and $\mathbf{r}$ dependent phase shift being added for all gradient pulses (see figure 5.1). As in the actual experiments, these phase 
shifts are summed over the ensemble to give an attenuation, the process being repeated for all desired values of $q_{u}$ and $q_{D}$.

The procedure outlined enables verification of the interpolation in $q_{D}$, the $q^{2}$ fitting in the $q_{u}$ dimension, the phase correction process and the validity of the superposition. The duration of each gradient pulse $\delta$ is not included in the simulation. Values for $q_{u}$ and $q$ are chosen to be similar to experiments. The effect of the finite encoding time, $\Delta_{u}$, for velocity is also included in the echo attenuation simulation, as shown above this can also be investigated with statistical calculations. In general the simulation noise due to the low number of tracer particles is larger than experimental noise. Nevertheless, a small amount of Gaussian noise is added to the simulated echo-attenuation before processing. The tensors calculated in this was are labelled in figure 5.10 as 'Simulated $E$ '. Overall the agreement between the simulated echo attenuation and the statistical calculation is excellent. Small discrepancies can be seen due to the approximation of the instantaneous velocity over the period $\Delta_{u}$, this effect is highlighted by the particles in the term $\left\langle v_{z}^{2} \bar{P}(Z, \tau)\right\rangle$ (see figure 5.10(i)) by the particles with no net displacement.

\subsection{Limits of Double-PGSTE Encoding}

Throughout this chapter we have interpreted each pair of the doublePGSTE experiment as a measurement of the instantaneous velocity. Here we examine the limits to which that is true.

In this section we treat the encoding for each gradient pulse as being instantaneous in time and that each pair of gradients will encode for displacement only. We will inspect the truncation errors associated with treating the result of the superposition as a true measure of $\langle u(0) u(\tau)\rangle$ or $\left\langle u^{2}\right\rangle$. Throughout this section we will treat all displacements as being centralised, that is, displacement with respect to the mean flow. In previous sections the encoding time $\Delta_{u}$ was included in the definition for $q_{u}$, here 

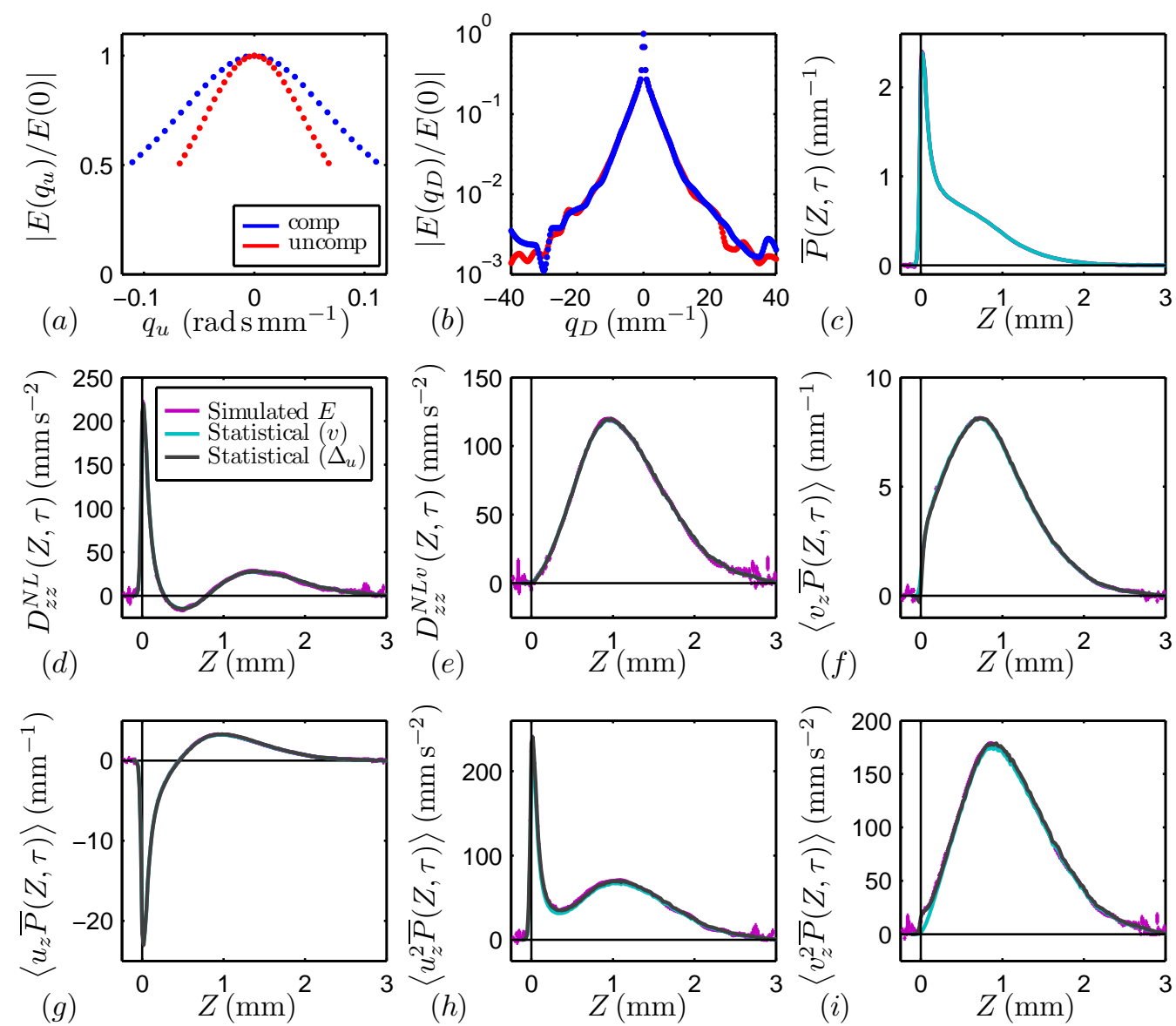

Figure 5.10: A set of simulated tracer particles were used to statistically calculate all the tensors that can be measured from an experiment where the motion and displacement encoding is parallel to the flow, panels (c) to (i). The velocity can be measured either from the instantaneous local velocity field, 'Statistical $(v)$ ', or as in experiment, over a finite encoding time 'Statistical $\left(\Delta_{u}\right)$ '. The same set of tracer particles were used to generate an echo attenuation function which was then processed in the manner described in the text. Panels (a) and (b) show the attenuation from the double-PGSE encoding and the displacement encoding. These tensors resulting from this analysis are labelled 'Simulated $E$ ' in panels (c) to (i). The agreement is excellent. 
we treat $\Delta_{u}$ as being the result of the fit to $q^{2}$.

\subsubsection{Velocity Autocorrelation Function}

Assuming that the fit to $q^{2}$ is free of truncation errors, the superposition will give an approximation of the velocity autocorrelation function $\langle u(0) u(\tau)\rangle_{\text {meas }}$ as

$$
\begin{aligned}
\langle u(0) u(\tau)\rangle_{\text {meas }} & \approx\langle u(0) u(\tau)\rangle \\
& =\frac{1}{\Delta_{u}^{2}}\left\langle\left(X\left(\Delta_{u}\right)-X(0)\right)\left(X\left(\tau+\Delta_{u}\right)-X(\tau)\right)\right\rangle .
\end{aligned}
$$

The displacement with respect to the mean flow of each tracer particle during the period $\Delta_{u}$ can be expressed as

$$
X\left(\Delta_{u}\right)-X(0)=\int_{0}^{\Delta_{u}} u(t) d t
$$

The correlations of displacements in equation 5.14 can then be written as

$$
\frac{1}{\Delta_{u}^{2}}\left\langle\left(X\left(\Delta_{u}\right)-X(0)\right)\left(X\left(\tau+\Delta_{u}\right)-X(\tau)\right)\right\rangle=\frac{1}{\Delta_{u}^{2}}\left\langle\int_{0}^{\Delta_{u}} u(t) d t \int_{\tau}^{\tau+\Delta_{u}} u\left(t^{\prime}\right) d t^{\prime}\right\rangle,
$$

so that the measured VACF can be expressed as the double integral over the true VACF

$$
\langle u(0) u(\tau)\rangle_{\text {meas }}=\frac{1}{\Delta_{u}^{2}} \int_{0}^{\Delta_{u}} \int_{\tau}^{\tau+\Delta_{u}}\left\langle u(t) u\left(t^{\prime}\right)\right\rangle d t d t^{\prime} .
$$

To test the limits of this expression we can use the Ornstein-Uhlenbeck expression for the longitudinal VACF [159], as a function of two time variables. Here we look at the practical limit of $\Delta_{u}<\tau$ so that in the expression below, $t^{\prime}>t$, this gives

$$
\left\langle u(t) u\left(t^{\prime}\right)\right\rangle=\left\langle u^{2}\right\rangle \exp \left(-\frac{\left(t^{\prime}-t\right)}{\tau_{c}}\right) .
$$


Putting this into the VACF approximation gives

$$
\langle u(0) u(\tau)\rangle_{\text {meas }}=\frac{1}{\Delta_{u}^{2}} \int_{0}^{\Delta_{u}} \int_{\tau}^{\tau+\Delta_{u}}\left\langle u^{2}\right\rangle \exp \left(-\frac{\left(t^{\prime}-t\right)}{\tau_{c}}\right) d t d t^{\prime} .
$$

Evaluating this integral gives

$$
\langle u(0) u(\tau)\rangle_{\text {meas }}=\frac{1}{\Delta_{u}^{2}}\left\langle u^{2}\right\rangle \tau_{c}^{2} \exp \left(-\frac{\tau}{\tau_{c}}\right)\left(\exp \left(\frac{\Delta_{u}}{\tau_{c}}\right)-1\right)\left(1-\exp \left(-\frac{\Delta_{u}}{\tau_{c}}\right)\right)
$$

Approximating the exponentials gives

$$
\begin{aligned}
\langle u(0) u(\tau)\rangle_{\text {meas }} & =\left\langle u^{2}\right\rangle \exp \left(-\frac{\tau}{\tau_{c}}\right)\left(1+\frac{\Delta_{u}^{2}}{12 \tau_{c}^{2}}\right) \\
& =\langle u(0) u(\tau)\rangle\left(1+\frac{\Delta_{u}^{2}}{12 \tau_{c}^{2}}\right)
\end{aligned}
$$

which suggests that the VACF measurement will be a slight overestimate and the approximation is valid if $\Delta_{u}<<12 \tau_{C}$. This condition is relatively easy to satisfy giving confidence in measurements of the VACF and hence $\mathrm{D}^{N L}$. Interestingly this condition does not depend on the measurement time $\tau$, as long $\Delta_{u}<\tau$.

\subsubsection{Second Moment of Velocity}

The superposition will also give the second moment of velocity $\left\langle u^{2}\right\rangle$, and the corresponding displacement resolved quantity. Expressing the measurement as a pure displacement encoding we have

$$
\left\langle u^{2}\right\rangle_{\text {meas }}=\frac{1}{\Delta_{u}^{2}}\left\langle(X-\bar{X})^{2}\right\rangle,
$$

here the displacement is being measured over the same interval so that the displacement due to diffusion can not be ignored, this gives 


$$
\left\langle u^{2}\right\rangle_{\text {meas }}=\frac{1}{\Delta_{u}^{2}}\left(2 D \Delta_{u}+\int_{0}^{\Delta_{u}} \int_{0}^{\Delta_{u}}\left\langle u(t) u\left(t^{\prime}\right)\right\rangle d d t^{\prime}\right) .
$$

Using the result of the Ornstein-Uhlenbeck integration 5.31 to give an expression for the mean squared displacement we get

$$
\left\langle u^{2}\right\rangle_{\text {meas }}=\frac{1}{\Delta_{u}^{2}}\left(2 D \Delta_{u}+2\left\langle u^{2}\right\rangle \tau_{c}\left(\Delta_{u}+\tau_{c}\left(\exp \left(-\frac{\Delta_{u}}{\tau_{c}}\right)-1\right)\right)\right)
$$

which gives, after approximating the exponential

$$
\left\langle u^{2}\right\rangle_{\text {meas }}=\frac{2 D_{0}}{\Delta_{u}}+\left\langle u^{2}\right\rangle\left(1-\frac{\Delta_{u}}{3 \tau_{c}}\right) .
$$

This sets an upper limit of the encoding time such that $\Delta_{u}<<3 \tau_{c}$. There is also a lower limit for the encoding time so that the diffusive displacement will start to dominate the apparent velocity measurement. Here we need

$$
\left\langle u^{2}\right\rangle>>\frac{2 D_{0}}{\Delta_{u}}
$$

If we are working in the Taylor regime and are inspecting the longitudinal displacements we have $\frac{D^{*}}{D_{0}} \approx \mathrm{Pe}$ and we saw in equation 5.32 the asymptotic dispersion of Ornstein-Uhlenbeck process is $\left\langle u^{2}\right\rangle \tau_{c}$, giving the inequality from equation 5.26 as

$$
\frac{\Delta_{u}}{\tau_{c}}>>\frac{1}{P e},
$$

in which case the choice of $\Delta_{u}$ must satisfy

$$
\frac{1}{P e}<<\frac{\Delta_{u}}{\tau_{c}}<<3
$$

Which can only be valid for large values of $P e$. Due to the approximate relationship between the $P e$ and $D_{0}$, the application of these limits must be taken with some care. The measurement of $\left\langle u^{2}\right\rangle$ will be an underestimate at large $\Delta_{u}$ and an overestimate at small $\Delta_{u}$. Our simulations show 
that even at large Pe $(<7000)$ the best choice of $\Delta_{u}$ will give a measurement of $\left\langle u^{2}\right\rangle$ that will have errors associated with diffusive effects and the finite integral over the VACF. This results in slight errors in the measured displacement resolved terms $\left\langle\bar{P}(X, \tau) u^{2}\right\rangle$ and $\left\langle\bar{P}(X, \tau) v^{2}\right\rangle$.

\subsection{Double PGSTE and Asymptotic Conditions}

The double PGSTE experiment, using the superposition of the compensated and uncompensated pulse sequences, gives a direct measurement of the velocity autocorrelation function. Previous methods, detailed in [16] and section 3.3.5 require the assumption of asymptotic conditions for extraction of the VACF. Using the superposition method to measure the VACF one can use the relationship given in equation 2.27 to monitor the approach to asymptotic conditions and quantify $\mathbf{D}^{*}(\tau)$.

Single PGSE experiments provide a direct measure of the mean-squared displacement and so also can provide details of the approach to asymptotic conditions. However due to the difficulty associated with taking derivatives of measured data the strict definition of dispersion

$$
\mathbf{D}^{*}(\tau)=\frac{1}{2} \frac{\partial \sigma^{2}}{\partial \tau}
$$

is not often used. Instead an effective or apparent dispersion is usually defined as

$$
\mathbf{D}_{e f f}^{*}(\tau)=\frac{1}{2 \tau} \sigma^{2}
$$

Assuming a system where the VACF behaves like a Ornstein-Uhlenbeck process [159] we can investigate the difference between the two cases. Omitting the pure diffusive term in the mean squared displacement, we can integrate the expression for the Ornstein-Uhlenbeck VACF twice to give

$$
\sigma^{2}(\tau)=2\left\langle u^{2}\right\rangle \tau_{c}\left(\tau+\tau_{c}\left(\exp \left(-\tau / \tau_{c}\right)-1\right)\right)
$$


and so using the definition in equation 5.29 the time dependent diffusion will grow as

$$
\mathbf{D}^{*}(\tau)=\left\langle u^{2}\right\rangle \tau_{c}\left(1-\exp \left(-\tau / \tau_{c}\right)\right) .
$$

this will asymptote very quickly to $\mathbf{D}^{*}=\left\langle u^{2}\right\rangle \tau_{c}$, to within $1 \%$ after $6 \tau_{c}$ and will initially be determined by

$$
\lim _{t \rightarrow 0} \mathbf{D}^{*}=\left\langle u^{2}\right\rangle \tau .
$$

In contrast the definition used in equation 5.30 for the effective dispersion will grow as [14]

$$
\mathbf{D}_{\text {eff }}^{*}(\tau)=\left\langle u^{2}\right\rangle \tau_{c}\left(1+\tau_{c} / \tau\left(\exp \left(-\tau / \tau_{c}\right)-1\right)\right)
$$

which will only be within $10 \%$ of $\left\langle u^{2}\right\rangle \tau_{c}$ after $10 \tau_{c}$ and will only be within $1 \%$ after $120 \tau_{c}$. See figure 5.11 . In the limit of short times we have

$$
\lim _{t \rightarrow 0} \mathbf{D}^{*}=\frac{1}{2}\left\langle u^{2}\right\rangle \tau .
$$

This slow approach makes determination of asymptotic conditions difficult and could potentially mask other physically relevant time scales. This is particularly relevant for transverse dispersion because the velocity autocorrelation function tends to show distinct features [16]. Direct measurement of the velocity autocorrelation function using the superposition technique, and subsequent integration, will preserve any temporal information and show a true approach to any asymptote.

\subsection{Conclusions}

Measurement of the nonlocal dispersion tensor requires a pair of twodimensional pulse sequences, each involving a single PGSTE component to measure displacements and hence correlation information, as well as a double PGTSE component to measure velocities. Data analysis involves a 


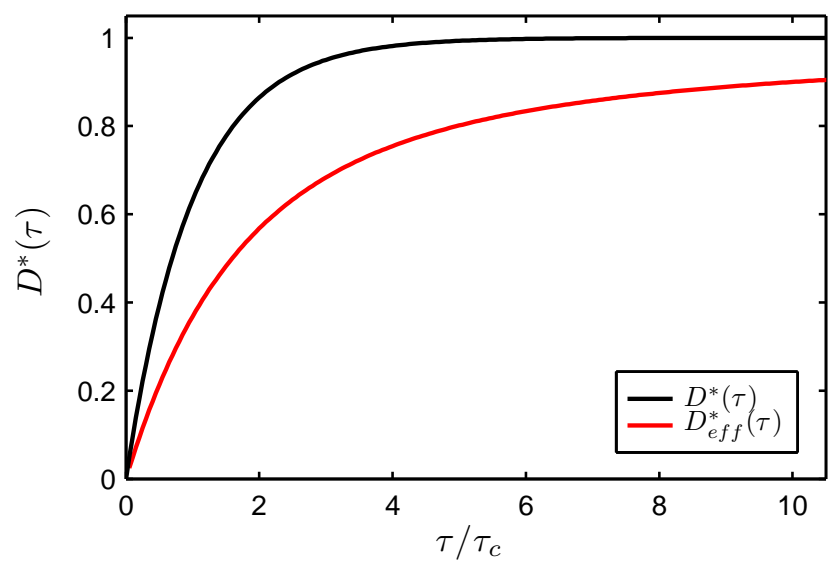

Figure 5.11: The approach to long time limit for a system with $\langle u(0) u(\tau)\rangle=$ $\left\langle u^{2}\right\rangle \exp \left(-\tau / \tau_{c}\right)$, where $\left\langle u^{2}\right\rangle=\tau_{c}=1$.

superposition of compensated and phase corrected uncompensated double PGTSE signals. This signal comparison often involves a small difference, requiring a consistent signal and hence a means of producing a stable flow. In the double PGTSE dimension a low- $q$ analysis is performed. An algorithm for reliably fitting data around $q=0$ for a wide range of attenuations is essential.

Components of the nonlocal dispersion tensor have been shown to be able to be measured with PGSE NMR. The robustness of our experimental protocols have been tested using lattice Boltzmann simulations. Statistically calculated tensor components obtained directly from tracer migration information in the lattice Boltzmann simulation are compared with those calculated by applying our data analysis protocols to simulated echo attenuation data also obtained from the lattice Boltzmann tracer information. Excellent agreement is found, thus further verifying our pulse sequence and processing methodology. The superposition required is also shown to be able to recover other nonlocal tensors, giving further information about the first and second moments of displacement. This pulse 
sequence is used to measure nonlocal dispersion in model systems (chapter 6), a full set of nonlocal dispersion coefficients for flow in a porous medium (chapter 7) and a selection of nonlocal components for flow in a Bentheimer rock core (chapter 9). 


\section{Chapter 6}

\section{Model Systems for Nonlocal Dispersion}

\subsection{Introduction}

In order to verify the experimental procedure used to measure components of the nonlocal dispersion tensor, we would require a system that can be measured and accurately modelled, preferably calculated analytically. To calculate the nonlocal dispersion tensor, we must know the complete Eulerian velocity field, $\mathbf{u}(\mathbf{r})$ and the joint probability, $P\left(\mathbf{r}, \mathbf{r}^{\prime}, \tau\right)$, of starting position and final position or displacement. A chosen component of the nonlocal dispersion tensor can then be written as

$$
D_{\alpha \beta}^{N L}\left(X_{\gamma}, \tau\right)=\int u_{\alpha}(\mathbf{r}, 0) P\left(\mathbf{r}, \mathbf{r}+X_{\gamma}, \tau\right) u_{\beta}\left(\mathbf{r}+X_{\gamma}, \tau\right) d \mathbf{r} .
$$

The calculation of such terms will begin to give us some insight into the way that nonlocal dispersion terms describe properties of the flow and correlations.

The challenge is to find examples where this is readily calculable, easy to simulate and able to be measured with NMR. These examples would also preferably show some structure to facilitate comparison. Three such 
examples will be presented here; first, Taylor dispersion for flow in capillary, second, Taylor dispersion in parallel plates and finally, flow of a Newtonian fluid in a Couette cell, in the absence of diffusion.

\subsection{Taylor Flow in a Capillary}

In steady-state laminar flow in the absence of diffusion the mean squared displacement will grow quadratically with time (see figure 2.5(d)). However in a regime where the diffusion is significant, the molecules will cross streamlines and in the long time limit have the opportunity to sample the entire velocity field. This will give a growth of the mean-squared displacement that is linear in time. The approach to asymptotic conditions for dispersion in a pipe flow has been well characterised $[54 ; 55]$. The long time limit is usually of the order of $a^{2} / D_{0}$ where $a$ is the tube radius and $D_{0}$ is the molecular self-diffusion coefficient. The longest time scale accessible with NMR is $\sim 2 \mathrm{~s}$, the upper limit being determined by nuclear spin relaxation time, this gives for water, an accessible displacement of $\sim 100 \mu \mathrm{m}$.

Despite the low signal to noise associated with using such a small sample, NMR measurements of dispersion in a capillary flow have been performed by Codd et al. in 1999 [98]. The solvent in this case was Octane, with a diffusion coefficient of $2.35 \times 10^{-9} \mathrm{~m}^{2} \mathrm{~s}^{-1}$ and the capillary diameter $150 \mu \mathrm{m}$. A flow rate of $2.6 \mathrm{~mm} \mathrm{~s}^{-1}$ was used. Figure 6.1 shows the measured and simulated longitudinal propagators for Taylor dispersion in Poiseuille flow. In the absence of diffusion the displacement propagator would simple be a hat function. At short times, as shown in panel (a), the propagator is broadened by diffusion, a significant fraction of the molecules have either moved backwards or have moved a displacement more than the maximum velocity would allow. At intermediate times, panel (b), a peak forms at displacement lower than the mean. This is because the molecules that are in the low velocity part of the flow, that is near the tube walls, can only diffuse into regions of higher velocity and hence 
experience a greater longitudinal displacement. As the encoding time becomes longer, the molecules in the fast moving regions diffuse away, resulting in the higher displacements becoming reduced (c), finally the propagator tends to a Gaussian profile (d) as the molecules tend to diffuse over the whole diameter of the capillary.

The simulation of displacement due to diffusion and velocity for flow in a capillary is relatively simple, the flow field being given directly by the Navier-Stokes equation and a simple random walk with perfectly reflecting cylinder walls. An implementation of this is detailed in section 6.2.3.

The transverse displacement for pure diffusion in cylindrical, and other geometries has been well studied in NMR, giving rise to the term "diffusivediffraction" [51; 160; 161]. At long times the echo attenuation in $q$-space becomes the fourier transform of the one-dimensional autocorrelation of the pore-space thus a PGSE experiment can directly probe the pore structure of a porous medium. PGSE experiments have been performed investigating the diffusive diffraction, and therefore been able to measure, and compare to calculation the transverse propagator due to a restricted diffusion.

\subsubsection{Calculation of Nonlocal Dispersion in Capillary Flow}

The measurements of Codd et al. [98] show that longitudinal dispersion can be measured in capillary flow, thus implying that a nonlocal component can also be measured. Such a component will have longitudinal velocity encoding, and to exploit the true diffusion driven dispersion, transverse displacement encoding. For fully developed Poiseuille flow the transverse displacement propagator for capillary flow can be calculated from the joint probability of diffusive displacement [50] given by, in cylindrical polar coordinates 


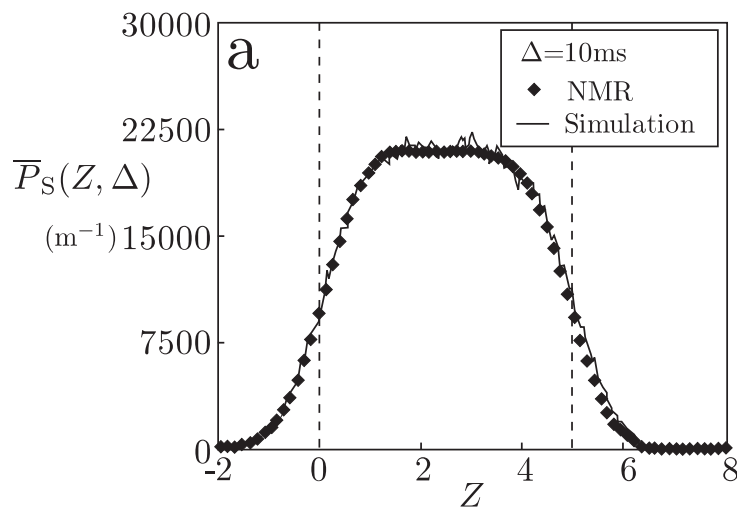

$\left(10^{-5} \mathrm{~m}\right)$

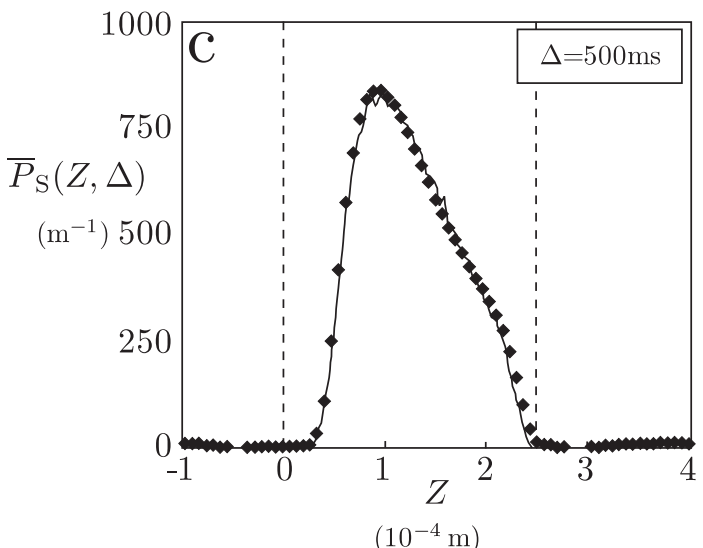

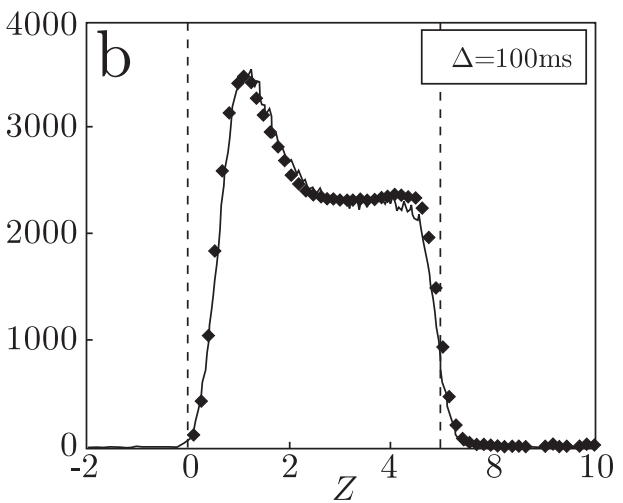

$\left(10^{-4} \mathrm{~m}\right)$

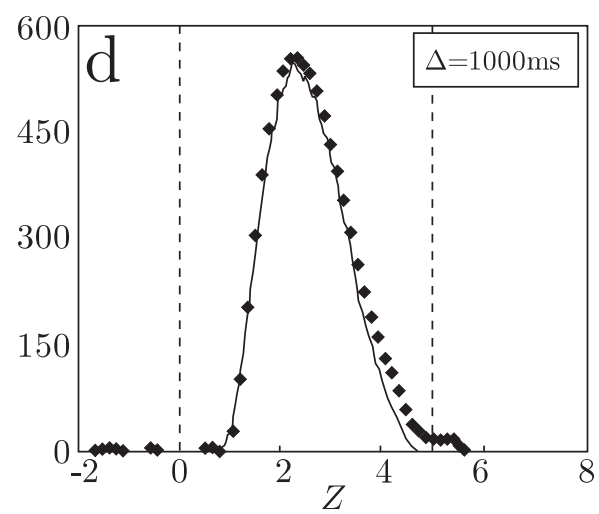

$\left(10^{-3} \mathrm{~m}\right)$

Figure 6.1: The longitudinal propagators as measured by Codd et al., using PGSE NMR. Figure adapted from [98]. Panel (a) shows the 'hat' function broadened by diffusion, in panel (b) the slower moving particles have bounced of the walls and begun to populate faster moving regions. Panels (c) and (d) show the propagator moving towards a Gaussian distribution. 
$P\left(\mathbf{r}, 0, \mathbf{r}^{\prime}, \tau\right)=\frac{1}{\pi a^{2}} \sum_{n=0, k=1}^{\infty} A_{n k}^{2} \exp \left(-\beta_{n k}^{2} \frac{D \tau}{a^{2}}\right) J_{n}\left(\beta_{n k} \frac{r}{a}\right) J_{n}\left(\beta_{n k} \frac{r^{\prime}}{a}\right) \cos n \theta \cos n \theta^{\prime}$

and has no dependance on $z$. The eigenvalues $\beta_{n k}$ can be found from

$$
\beta_{n k} \frac{J_{n}^{\prime}\left(\beta_{n k}\right)}{J_{n}\left(\beta_{n k}\right)}=\frac{-M a}{D},
$$

where $M$ is the relaxivity. Here the relaxivity is taken to be zero giving $\beta_{n k}$ such that

$$
J_{n}^{\prime}\left(\beta_{n k}\right)=0 .
$$

The coefficient $A_{n k}^{2}$ in equation 6.2 is given by

$$
\begin{aligned}
A_{n k}^{2} & =\frac{2}{\pi a^{2}} \frac{\beta_{n k}^{2} / J_{n}^{2}\left(\beta_{n k}\right)}{\beta_{n k}^{2}-n^{2}} \quad \text { for } n \neq 0 \\
& =\frac{1}{\pi a^{2}} \frac{1}{J_{0}^{2}\left(\beta_{0 k}\right)} \quad \text { otherwise. }
\end{aligned}
$$

Equation 6.2 being in cylindrical polar coordinates does not lend itself for construction of a nonlocal dispersion term as given by equation 6.1. Instead the Fourier Transform in the displacement dimension is calculated. This gives the nonlocal dispersion tensor component, $\hat{D}_{z z}^{N L}(\mathbf{q}, \tau)$, as a function of q. Thus we have

$$
\hat{D}_{z z}^{N L}(\mathbf{q}, \tau)=\iint u_{z}(\mathbf{r}) P\left(\mathbf{r}, \mathbf{r}^{\prime}, \tau\right) u_{z}\left(\mathbf{r}^{\prime}\right) \exp \left(i 2 \pi \mathbf{q} \cdot\left(\mathbf{r}^{\prime}-\mathbf{r}\right)\right) d \mathbf{r} d \mathbf{r}^{\prime}
$$

The nonlocal dispersion term, and its Fourier Transform, is naturally expressed in Cartesian coordinates, however the integral above must be over all starting and finishing positions and this is now conveniently performed in cylindrical polar coordinates. We are free to choose a Cartesian direction 
of $\mathbf{q}$, this gives the projection of the cylindrical geometry onto a Cartesian plane, as in an experiment measurement. This gives

$$
\begin{aligned}
\hat{D}_{z z}^{N L}(q, \tau)= & \int_{0}^{a} \int_{0}^{2 \pi} \int_{0}^{a} \int_{0}^{2 \pi} \bar{v}\left(1-2 \frac{r^{2}}{a^{2}}\right) \frac{1}{\pi a^{2}} \sum_{n, k} A_{n k}^{2} e^{-\beta_{n k}^{2} \frac{D \tau}{a^{2}}} J_{n}\left(\beta_{n k} \frac{r}{a}\right) \times \\
& J_{n}\left(\beta_{n k} \frac{r^{\prime}}{a}\right) \cos n \theta \cos n \theta^{\prime} \bar{v}\left(1-2 \frac{r^{\prime 2}}{a^{2}}\right) e^{i 2 \pi q r \cos \theta-i 2 \pi q r^{\prime} \cos \theta^{\prime}} \\
& r d \theta d r r^{\prime} d \theta^{\prime} d r^{\prime},
\end{aligned}
$$

due to the symmetry of the coordinates, this can be written as

$$
\begin{aligned}
\hat{D}_{z z}^{N L}(q, \tau)= & \frac{\bar{v}^{2}}{\pi a^{2}} \sum_{n, k} A_{n k}^{2} e^{-\beta_{n k}^{2} \frac{D \tau}{a^{2}}} \times \\
& \left|\int_{0}^{a} \int_{0}^{2 \pi}\left(1-2 \frac{r^{2}}{a^{2}}\right) J_{n}\left(\beta_{n k} \frac{r}{a}\right) \cos n \theta e^{i 2 \pi q r \cos \theta} r d r d \theta\right|^{2} .
\end{aligned}
$$

From the definition of Bessel functions we have.

$$
J_{n}(x)=\frac{1}{i^{n} 2 \pi} \int_{0}^{2 \pi} e^{i x \cos \theta} e^{i n \theta} d \theta
$$

and since $J_{-n}(x)=(-1)^{n} J_{n}(x)$,

$$
J_{n}(x)=\frac{1}{i^{n} 2 \pi} \int_{0}^{2 \pi} e^{i x \cos \theta} e^{-i n \theta} d \theta .
$$

This enables the integral over $\theta$ to be evaluated giving,

$$
\begin{aligned}
\hat{D}_{z z}^{N L}(q, \tau)= & \frac{\bar{v}^{2}}{\pi a^{2}} \sum_{n, k} A_{n k}^{2} e^{-\beta_{n k}^{2} \frac{D \tau}{a^{2}}} \times \\
& \left|\int_{0}^{a}\left(1-2 \frac{r^{2}}{a^{2}}\right) J_{n}\left(\beta_{n k} \frac{r}{a}\right) i^{n} 2 \pi J_{n}(2 \pi q r) r d r\right|^{2}
\end{aligned}
$$

From here we proceed numerically, the integral over $r$ was performed using Simpson's rule over 101 steps while the first 12 indices were used 
for $n$ and $k$. A numerical fourier transform was used to recover $D_{z z}^{N L}(X, \tau)$. These results are shown in figure 6.2 for encoding times of $0.1,0.2,0.5,1$ and 2 in units of $\frac{a^{2}}{D}$. Further discussion of these terms are in section 6.2.2.

The expression for the fourier transform of the pure diffusive propagator, or equivalently, the PGSE echo attenuation, can be constructed without the $v(r)$ term. In this case the integral over $r$ is readily solvable and given in [50].
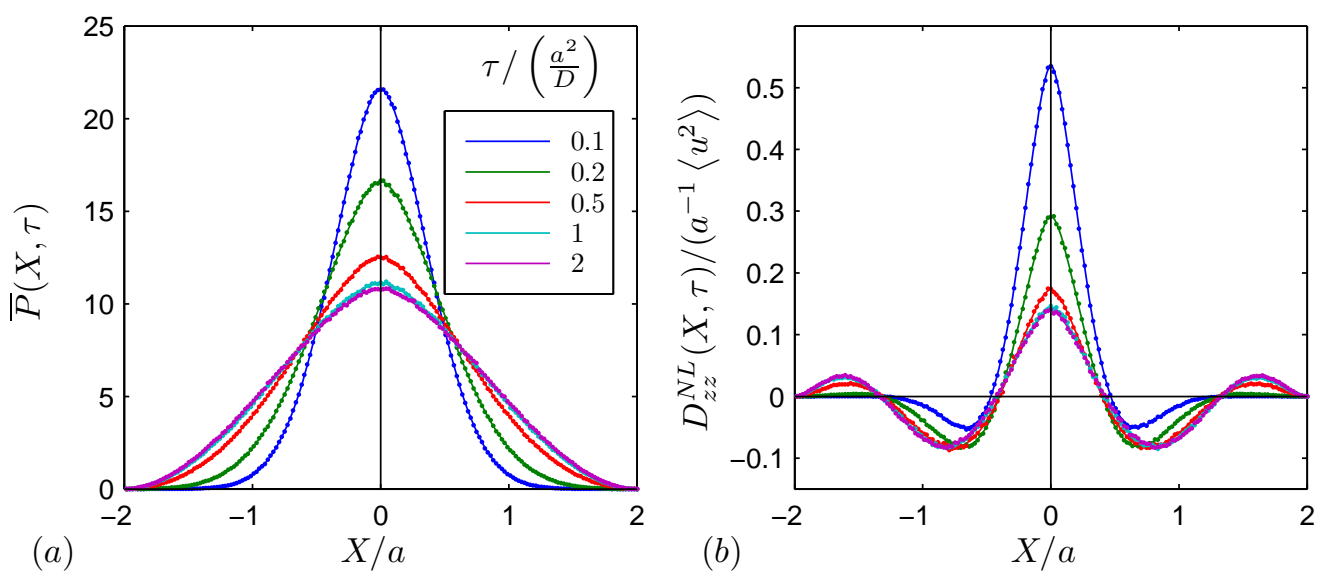

Figure 6.2: The propagator (a) and the nonlocal dispersion component (b) $D_{z z}^{N L}(X, \tau)$ for flow in a capillary at a range of encoding times. The dots show the statistical calculation, the lines show the results from the numerical integrations.

\section{Velocity Autocorrelation Function}

The integral of the nonlocal dispersion component $D_{z z}^{N L}(X, \tau)$ over all displacement should directly give the velocity autocorrelation function. Since we have have an expression for the Fourier inverse of the nonlocal dispersion tensor, setting $q=0$ will give this correlation. In this case only $n=0$ and $k \geq 2$ remain. 


$$
\begin{aligned}
\langle u(0) u(\tau)\rangle= & \frac{\bar{v}^{2}}{\pi a^{2}} \sum_{k} A_{0 k}^{2} e^{-\beta_{0 k}^{2} \frac{D \tau}{a^{2}}} \times \\
& \left|\int_{0}^{a}\left(1-2 \frac{r^{2}}{a^{2}}\right) J_{0}\left(\beta_{0 k} \frac{r}{a}\right) i^{n} 2 \pi r d r\right|^{2} .
\end{aligned}
$$

Making use of the standard integrals

$$
\begin{aligned}
\int_{0}^{a} r^{3} J_{0}(\alpha r) d r & =\frac{a^{3}}{\alpha} J_{1}(\alpha a)-\frac{2 a^{2}}{\alpha^{2}} J_{2}(\alpha a), \quad \text { for } \quad \alpha \neq 0 \\
& =\frac{a^{4}}{4}, \quad \text { for } \quad \alpha=0,
\end{aligned}
$$

and

$$
\begin{aligned}
\int_{0}^{a} r J_{0}(\alpha r) d r & =\frac{a}{\alpha} J_{1}(\alpha a), \quad \text { for } \quad \alpha \neq 0 \\
& =\frac{a^{2}}{2} \quad \text { for } \quad \alpha=0,
\end{aligned}
$$

and for $n=0$ the values of $\beta_{0 k}$ can now be expressed as

$$
J_{1}\left(\beta_{0 k}\right)=0
$$

This simplifies the expression for the velocity autocorrelation function to [54]

$$
\langle u(0) u(\tau)\rangle=\sum_{k=2}^{\infty} \bar{v}^{2} \frac{64}{\beta_{0 k}^{4}} e^{-\beta_{0 k}^{2} D \tau / a^{2}} .
$$

The velocity autocorrelation function, both calculated and simulated is shown in figure 6.3. This correlation function is dominated by the $k=2$ component, giving a correlation time of $\frac{a^{2}}{\beta_{02}^{2}}=\frac{0.0681 a^{2}}{D}$. This suggests a rapid decay of correlation, and indeed after 10 correlation times the simulated data is in the noise. In contrast at this time the nonlocal dispersion term $D_{z z}^{N L}(X, \tau)$ is still developing structure, thus showing further displacement correlations. For an encoding time of $2 a^{2} / D$, that is, almost 

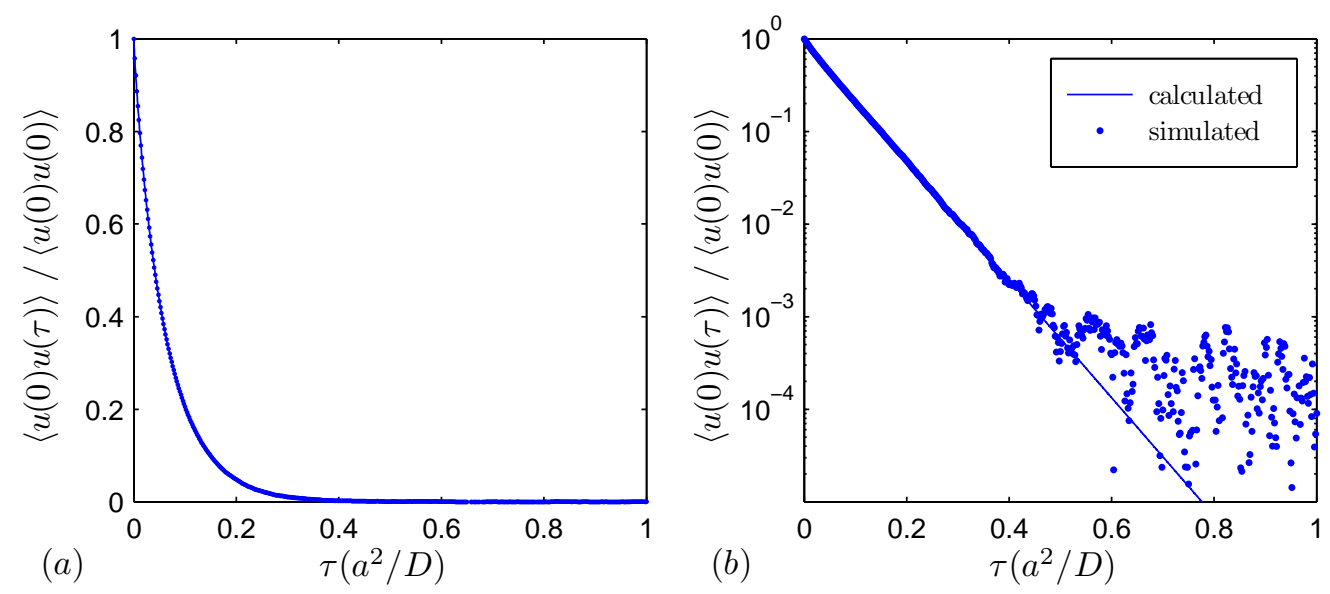

Figure 6.3: The calculated (solid line) and simulated (dots) longitudinal velocity autocorrelation function for dispersion in a capillary. The same data has been plotted on a linear scale (a) and a log-linear scale (b). The log-linear graphs shows the simulation noise at a time of $\sim 0.5 \frac{a^{2}}{D}$. The apparent mono-exponential decay is actually a series of decays (see equation 6.17), dominated by a decay time of $\frac{0.0681 a^{2}}{D}$.

30 correlation times, we have just begun to see the asymptote in the nonlocal dispersion. This highlights the extra information that can be seen in the nonlocal dispersion tensor when the velocity autocorrelation function has decayed to zero.

\subsubsection{Inferring Properties of the Flow}

The nonlocal component $D_{z z}^{N L}(X, \tau)$ provides a useful example to enable inferences of the flow properties. In this case we do have a complete description of the flow and diffusion driven transport, but interpreting the nonlocal dispersion components provides a useful exercise. Here we consider the influence of individual particles and how they contribute to the component. There are obvious difficulties in using a handful of examples to try and describe a true ensemble averaged quantity. Nevertheless doing so highlights the ability of the nonlocal dispersion tensor to quantity and 
qualify average behaviour.

Referring to figure 6.4, at short times, $0.1 a^{2} / D$ we see large peak around a displacement of zero. This is due to the large number of particles, typified by the particle $A$ that have only displaced short distances in this relatively short time. These particles will be sampling the same initial and final velocity. A strong anticorrelation can also be seen for particles that have moved $\pm 0.7 \mathrm{a}$, even though the propagator shows that the proportion of these particles is small. These particles, such as $D$, on average, have a large difference between their final and initial velocity and will have moved from a region moving faster than the mean, to a region that is moving slower. Note that this is a one-dimensional projection of the ensemble average, there will still be a number of particles with a displacement of $\pm 0.7 a$ that have a high degree of correlation. The particles that have displaced by $\pm 0.4 a$, such as $B$ and $C$, will on average have no correlation.

At long times, $\tau=2 a^{2} / D$, with reference to figure 6.5, the anticorrelation lobes become more pronounced as particles such as $C$ become prevalent. Further correlation lobes appear, centered around $\pm 1.6 a$. These correspond to particles such as $B$ that have, in the encoding time $\tau$ moved from one side of the tube to the other. As a result each particle's final and initial velocity are correlated. This structure will persist at infinite time.

\subsubsection{Simulation of Nonlocal Dispersion in Capillary Flow}

A simple random walk and velocity flow for an ensemble of particles can be implemented to statistically calculate the nonlocal dispersion components. For each tracer particle the initial position and later positions at pre-defined intervals is recorded. From these positions the velocity can be calculated. The propagator is then built up simply from a histogram of the displacements. In order to calculate the nonlocal dispersion tensor component, the contribution to the velocity autocorrelation from each tracer 


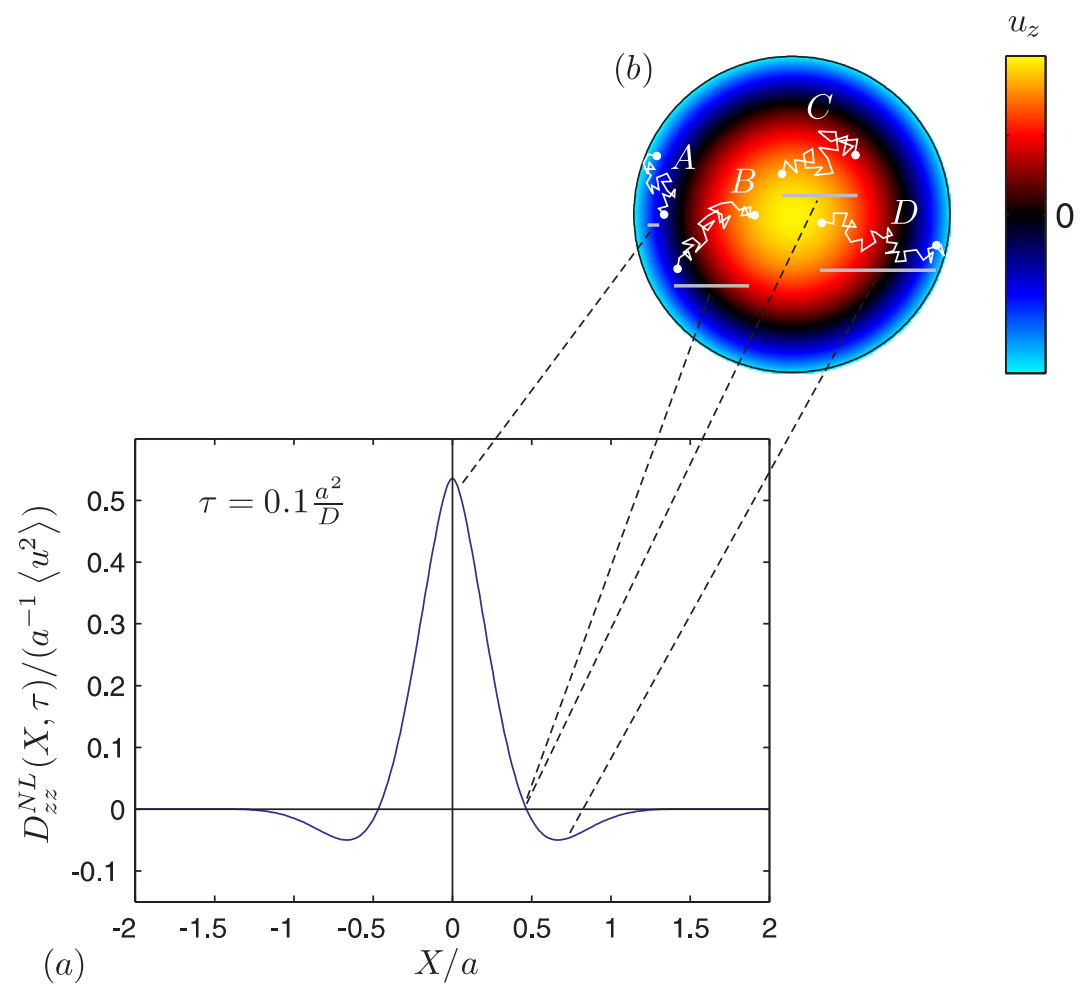

Figure 6.4: The calculated component $D_{z z}^{N L}(X, \tau)$ for $\tau=0.1 \frac{a^{2}}{D}$, (a). The velocity distribution in the capillary is shown in (b). The particle $A$ has a high degree of correlation and contributes to the region near $X=0$. The particle $D$ has moved between a region above the mean to below the mean, thus giving an anticorrelation. Particles $B$ and $C$ have the same $X$ displacement but different correlations. These particles have been denoted with a positive displacement, but the symmetry is apparent.

particle can be calculated and then put into a bin depending on how far it has moved in the chosen direction. The accumulation of tracer particles will statistically build up the desired quantity.

The walls can be assumed to be perfectly reflecting. Calculations and experiments have been performed for enhanced relaxtion at the walls but this won't be considered here. Each diffusive step must be checked to see if the step would result in the tracer particle being outside the cylinder wall. If this is the case, the particle is reflected back. 


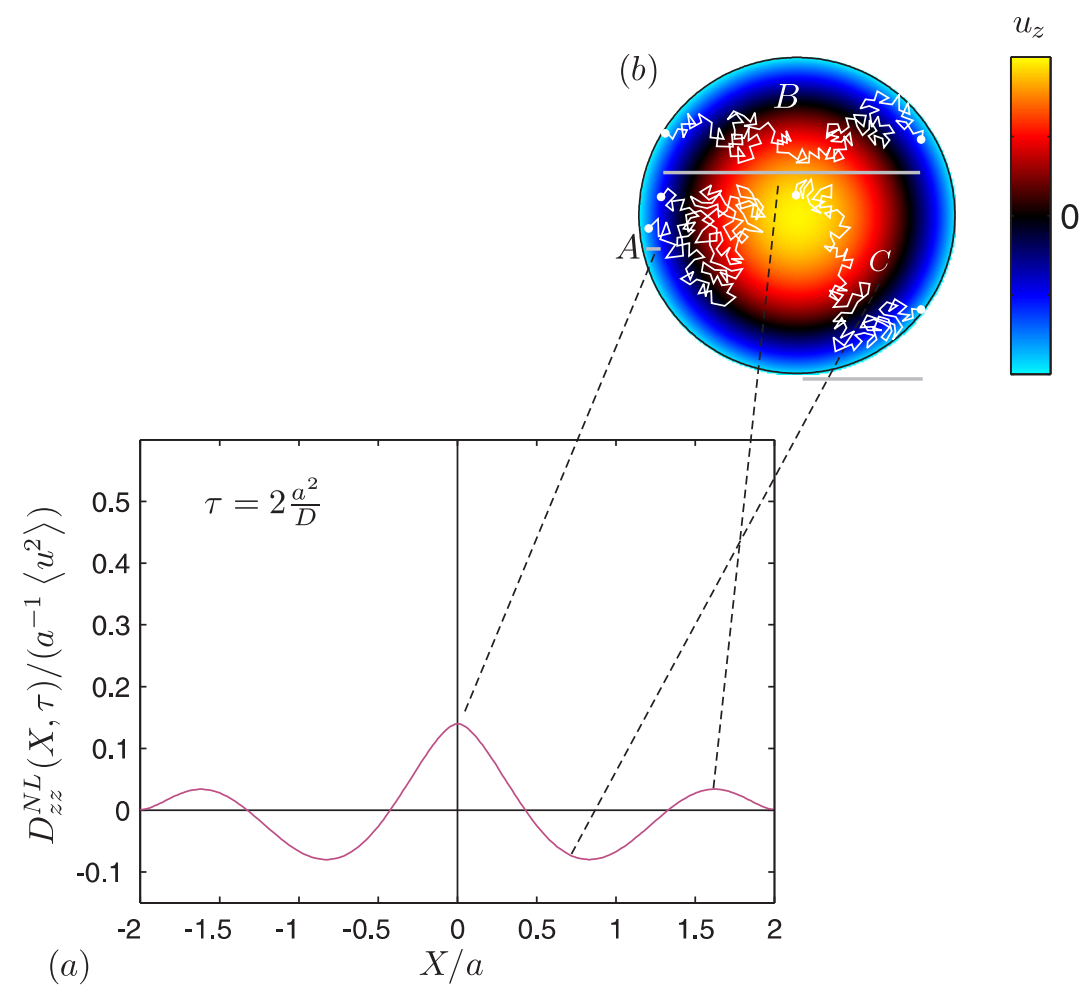

Figure 6.5: The calculated component $D_{z z}^{N L}(X, \tau)$ for $\tau=2 \frac{a^{2}}{D}$, (a). The velocity distribution in the capillary is shown in (b). The particle $A$ has returned close to its original location with a high degree of correlation and contributes to the region near $X=0$. The particle $B$ has moved almost a complete diameter, in order for particles to do this they will move between regions of similar velocities, thus giving the correlation. Particles $C$ shows an example of a particle that has displaced such that at anticorrelation results. These particles have been denoted with a positive displacement, but the symmetry is apparent.

The simulation time is divided up into units of $t_{s}$ so that the discrete diffusion steps are on average $<0.05 \mathrm{a}$. For each diffusion step each particles position is updated via

$$
\begin{aligned}
& x=x+w_{x} \\
& y=y+w_{y} \\
& z=z+w_{z}+v(x, y) t_{s},
\end{aligned}
$$




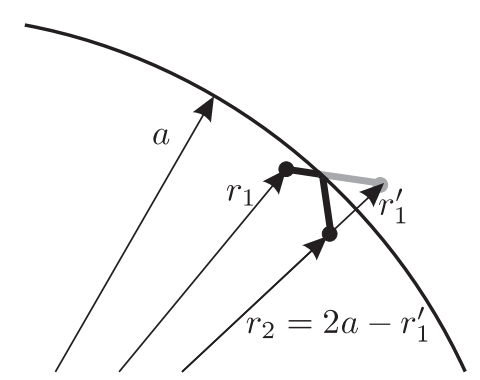

Figure 6.6: The reflection of a tracer particle from a cylindrical barrier. The step size needs to be small compared to the cylinder radius $a$.

where $w_{x}, w_{y}$ and $w_{z}$ are independent random jumps with Gaussian distribution with a standard deviation of $\sqrt{2 D t_{s}}$.

The approximation to the reflection is described in figure 6.6. A particle at a radius of $r_{1}$ is given a diffusion step, if the tracer particle's new radius $r_{1}^{\prime}$ is greater than the cylinder radius $a$, the particle is reflected back giving a new radius of $r_{2}=2 a-r_{1}^{\prime}$. All other coordinates remain the same. The diffusion steps and flow steps are then able to continue. Typical simulations will use $2 \times 10^{6}$ tracer particles.

The component $D_{z z}^{N L}(X, \tau)$ and the propagator $P(X, \tau)$ for a range of encoding times is shown, with direct comparison to calculation in figure 6.2. The agreement verifies the statistical method of calculating the nonlocal dispersion tensor. The velocity autocorrelation function, also compared to calculation, is shown in figure 6.3. These quantities are discussed in section 6.2.2.

The simulation allows for convenient investigation of other nonlocal terms. For capillary flow we also have the term $D_{z z}^{N L}(Z, \tau)$. Since this term, and the longitudinal propagator, will cover a broad range of displacements, it is more natural to express this component in terms of velocity, $V_{z}$. The propagator $P\left(V_{z}, \tau\right)$ and the nonlocal dispersion component $D_{z z}^{N L}\left(V_{z}, \tau\right)$ are shown in figure 6.7a and 6.7b respectively. Note in the propagator the agreement with the experimental literature data from figure 6.1, 
although in simulation we explore slightly different encoding times. In stark contrast to the component $D_{x x}^{N L}(X, \tau)$, the component $D_{z z}^{N L}\left(V_{z}, \tau\right)$ dies away very quickly and little structure remains at an encoding time of $2 a^{2} / D$.

Continuing with our interpretation in section 6.2.2, we can also infer properties of the flow from the component $D_{z z}^{N L}\left(V_{z}, \tau\right)$. The asymmetry of the correlation peaks at short times can be explained by inspecting the propagator $P\left(V_{z}, \tau\right)$. As mentioned in section 6.2, the particles with a large negative velocity, near the wall, will reflect off the walls and move to a region of different velocity, thus reducing the net correlation. Initially the particles near the center of the tube will be diffusing in a region with a small velocity gradient with a higher degree of correlation. As these fast moving particles diffuse further the correlation rapidly decreases.
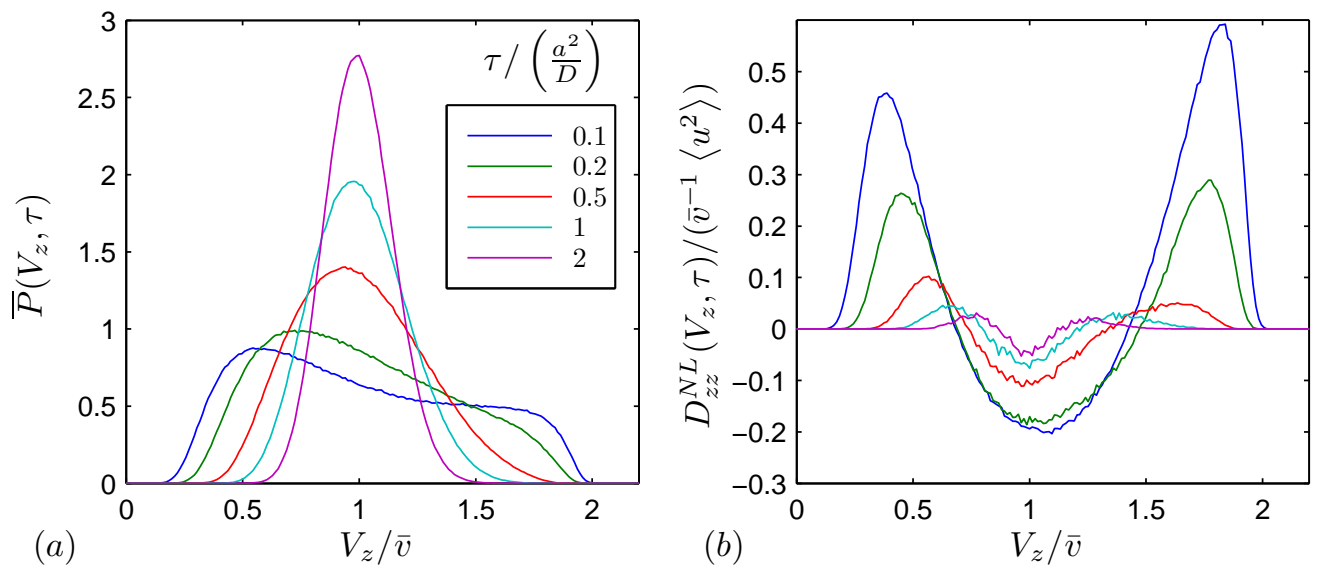

Figure 6.7: The simulated propagator (a) and the nonlocal dispersion component (b) $D_{z z}^{N L}\left(V_{z}, \tau\right)$ for flow in a capillary. Encoding times are in units of $\frac{a^{2}}{D}$ and the mean velocity, $\bar{v}$, is $200 \frac{D}{a}$

The large anticorrelation peak around the mean velocity describes a subset of particles that have moved from a region with a velocity lower than the mean to a region with a velocity higher than the mean, or vice versa. For a given time, these particles have a displacement equal to the 
mean, they are not however, a subset of particles travelling at the mean velocity.

\subsubsection{Experimental Measurement of Nonlocal Dispersion in Capillary Flow}

Here we present the first experimental results of the thesis. This experiment was technically the most challenging and the results were modest. As a method for verifying the pulse sequence it was found to be unsuitable. As will be shown later, the experiments on a Couette cell more than fulfill this role and as such these experiments for the capillary flow were not investigated exhaustively. Nevertheless the results on the capillary flow work are presented below.

\section{Simultaneous Longitudinal and Transverse Encoding}

Experiments were performed under similar conditions to that described in figure 6.1 , in this case a capillary of diameter, $2 a=100 \mathrm{um}$ was used with water with a tube velocity of $1.8 \mathrm{~mm} \mathrm{~s}^{-1}$.

We have seen that PGSE-NMR has successfully been used to separately measure longitudinal dispersion and, in the case of no mean flow transverse displacement. Combining these two measurements is not trivial, one must take care to precisely line up the capillary with the gradient geometry. In the given encoding time the mean diffusion length will be $45 \mu \mathrm{m}$ and if the capillary is mis-aligned by $2.5^{\circ}$ the observed transverse displacement due to the bulk flow will be $40 \mu \mathrm{m}$.

The small size of the capillary results in a low signal to noise, in order to improve this a smaller, more sensitive $B_{1}$ coil was wound directly onto a capillary. The coil consisted of 20 turns of $0.1 \mathrm{~mm}$ diameter wire with each turn separated by a keratin thread of approximately $70 \mu \mathrm{m}$ diameter resulting in a coil of length $3.6 \mathrm{~mm}$. In contrast to later results in this thesis, these measurements were performed at $300 \mathrm{MHz}$. Imaging techniques 
were used to measure the angle of the capillary with respect to the gradient field. The gradient field can then be rotated for subsequent experiments.

As will be detailed in later sections, the stability of the flow over a wide range of time scales is important in order to obtain reliable data. The attenuation in the $q_{u}$ direction is small and any variation in velocity will appear as noise-like artifacts, making analysis difficult. In this experiment an HPLC pump (Pharmacia P-500) was modified into a pure syringe pump. An alternative stepper motor was used, an adapted Rheo-NMR motor employing continuously driven steps. This was reduced down by a 500:1 box and then driven into the existing workings of the pump. A stepper motor setting of $0.15 \mathrm{~Hz}$ drove the $10 \mathrm{~mm}$ diameter piston at $180 \mathrm{~nm} \mathrm{~s}^{-1}$ giving a tube velocity of $1.8 \mathrm{~mm} \mathrm{~s}^{-1}$ in the capillary. A mass displacement pumping system would usually result in a steady flow, however the gradual formation of air bubbles in the flow lines over several hours meant that only modest stability was achieved. The presence of air bubbles introduces some compressibility in the flow lines, resulting in a slow variation of the average flow rate.

The experiment was performed with 10 steps in the displacement directions. The signal-to-noise ratio limited the displacement resolution to $22.2 \mu \mathrm{m}$ and the maximum measured displacement was chosen to be $2 a$. In the motion encoding dimension, 33 steps were used. The double-PGSE gradients where separated by $3 \mathrm{~ms}$. The total experiment time, for both the compensated and uncompensated pulse sequence was 11 hours. In order to measure a term with significant structure an encoding time of $500 \mathrm{~ms}$ was chosen.

The measurement requires the superposition from two pulse sequences, each pulse sequence has an encoding for the propagator, when $q_{u}=q_{c}=0$. Comparing the two independently acquired propagators provides a measure of experimental uncertainty, and in this case, another means for comparing experimental results with calculation. Figure 6.8(a) shows the calculated propagator and the measured propagators from the uncompen- 

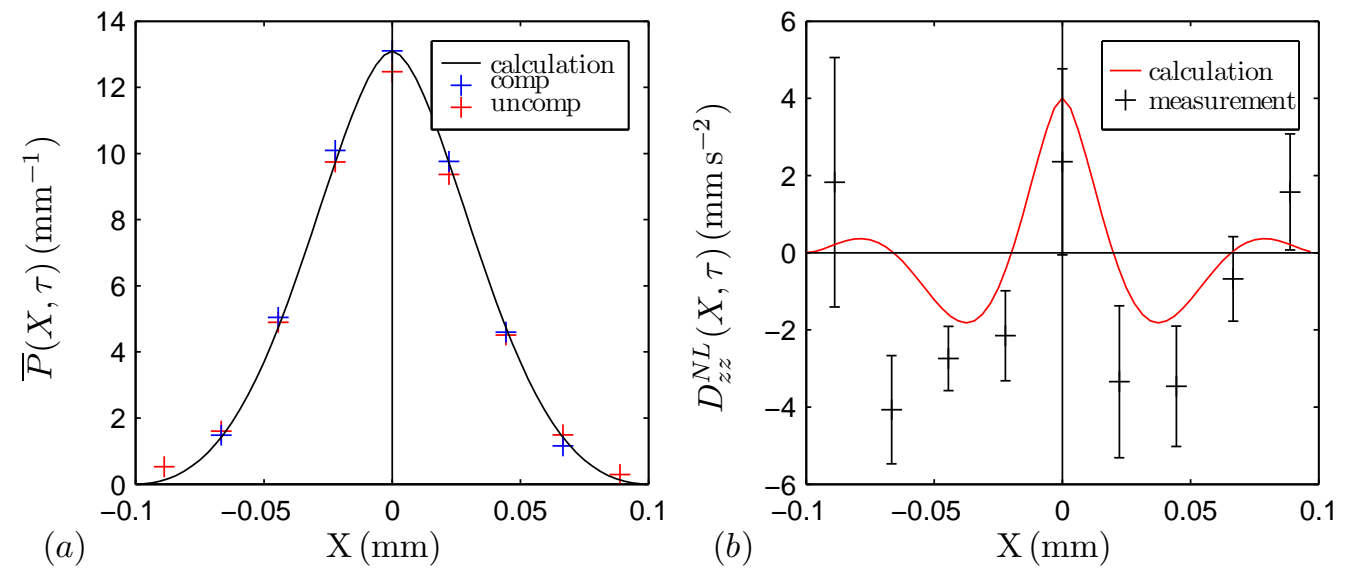

Figure 6.8: The measured (crosses) and calculated (black line) transverse displacement propagator for capillary flow (a) with an encoding time of $\tau=500 \mathrm{~ms}$. The blue and red crosses show the measurement from the compensated and uncompensated measurements respectively. Panel (b) shows the nonlocal term $D_{z z}^{N L}(X, \tau)$ measured (black crosses) and simulation (red line). The agreement in the propagator is good and is modest for the nonlocal dispersion, without being convincing. 
sated and compensated experiments. The agreement with the theory is satisfactory. We shall see in later results, an improvement between the agreement of the two separate propagator measurements is sought after for quality measurements of the nonlocal dispersion tensor. Figure 6.8(b) shows the measured component $D_{z z}^{N L}(X, \tau)$ for $\tau=500 \mathrm{~ms}$. The error bars for each displacement pixel are given from the low- $q$ fit described in section 5.4.2. There is a general agreement with the theory and some evidence of an anticorrelation for particles than have displaced by $\sim 50 \mu \mathrm{m}$. The magnitude of the measurement is consistent with the calculation. However, the large error bars precludes further conclusions and hence we do not consider this measurement to be a suitable verification of our experimental technique.

\subsubsection{Further Improvements}

The component $D_{z z}^{N L}(X, \tau)$ for Taylor flow in a capillary is desirable to measure as it offers the potential for direct verification of an experiment measurement via comparison with an analytical expression. This also has the feature of being a diffusion driven dispersive system. While the results above are modest, there are a number of ways, in the future, that the experiment can be improved.

\section{Multi-Capillary Arrays}

One real power of PGSE techniques is the ensemble averaging [17], enabling experimental optimisation of signal-to-nose and the probing of small displacements. In this case, an experiment with $N$ identical capillaries in parallel would give the same result, with $N$ times more signal. One could construct an array of 10-50 capillaries for detection in a standard $B_{1}$ coil. The gain in signal to noise would be further multiplied by the increase in length, offered by a longer excitation volume. The difficulty in this experiment would be construction of the array such that all the capillaries are 
parallel, it is essential that all the transverse displacement is purely due to diffusion, rather than a component of the longitudinal velocity in a misaligned capillary. In addition, depending on the entry and exit of the capillary array, the average velocity in each capillary may differ. The capillaries at the edges of the array will have a slightly reduced average velocity. This could be compensated for by constructing an array with shorter capillaries at the outside, or by allowing for this in the theory. Such an approach has not been pursued.

\section{Other Geometries}

The greatest structure in the nonlocal component is seen at longer times, especially when particles have completely traversed the capillary. The geometry of the capillary means that only a small fraction of particles will travel a diameter. A channel with a square cross section would provide a greater proportion of large transverse displacements. Micro-channel fabrication techniques in polydimethylsiloxane [162] could provide a method for constructing a large array of such channels. An analytical expression for the nonlocal dispersion could be found, the flow field being given by a Fourier series solution to the Navier-Stokes equation. The expression for the transverse displacement can be found in [50], and is repeated here. For convenient comparison with the cylindrical expression, the width of the channel is taken as $2 a$

$$
\begin{aligned}
P\left(z, 0, z^{\prime}, \tau\right)= & a^{-1} \sum_{n=0} \exp \left(-\xi_{n}^{2} \frac{D \tau}{a^{2}}\right) \frac{\cos \xi_{n} z / a \cos \xi_{n} z^{\prime} / a}{1+\operatorname{sinc} 2 \xi_{n}} \\
& +a^{-1} \sum_{m=0} \exp \left(-\zeta_{n}^{2} \frac{D \tau}{a^{2}}\right) \frac{\cos \zeta_{m} z / a \cos \zeta_{m} z^{\prime} / a}{1+\operatorname{sinc} 2 \zeta_{n}}
\end{aligned}
$$

where the value $\xi_{n}$ and $\zeta_{m}$ are determined by,

$$
\xi_{n} \tan \xi_{n}=\frac{M a}{D}
$$


and

$$
\zeta_{m} \cot \zeta_{m}=-\frac{M a}{D}
$$

A further improvement to this experiment would be to construct a monolith of parallel plates. Aside from the difficulty of ensuring identical flow within each pair of plates, this would have the advantages of large signal to noise ratio since the sample is continuous (to a limit) in one transverse direction. In addition, due to the two-dimensional nature of the flow profile, only displacements in the transverse encoding direction will create anticorrelations. This means that the correlation effects, either in the pure VACF or resolved into a nonlocal dispersion component, will be stronger and hence easier to measure. Figure 6.9 shows simulations of the longitudinal velocity autocorrelation function and the transverse propagator for flow in a capillary and parallel plate, both simulations were performed with $D=2.5 \times 10^{-3} \mathrm{~mm}^{2} \mathrm{~s}^{-1}, a=0.05 \mathrm{~mm}$ and $\bar{v}=10 \mathrm{~mm} \mathrm{~s}^{-1}$. The correlation function, figure 6.9(a), is initially stronger for the capillary flow, due to the larger distribution of velocities about the mean. However the dominant decay for the parallel plate flow is significantly slower. The propagator, for an encoding time of $500 \mathrm{~ms}$, in figure 6.9 highlights the fact that in the parallel plate, there are more than double the number of particles that have moved $\pm 0.16 \mathrm{a}$. This larger proportion of particles directly results in a larger signal to noise ratio in the $q_{u}$ dimension of the corresponding displacement pixel.

In addition to a parallel plate geometry providing a better signal to noise ratio, there is less demand on the accuracy associated with superposition. The superposition required for the component $D_{z z}^{N L}(X, \tau)$ for both capillary flow and parallel plate flow is shown in figure 6.10. In the case of parallel plate flow, the nonlocal dispersion tensor component is stronger, and the differences between the $q^{2}$ fit of the compensated and uncompensated experiments are much larger. The correlation lobe for large displacements is larger and there is also a strong feature at $X=0$. For future experiments where comparison is required between calculation and ex- 

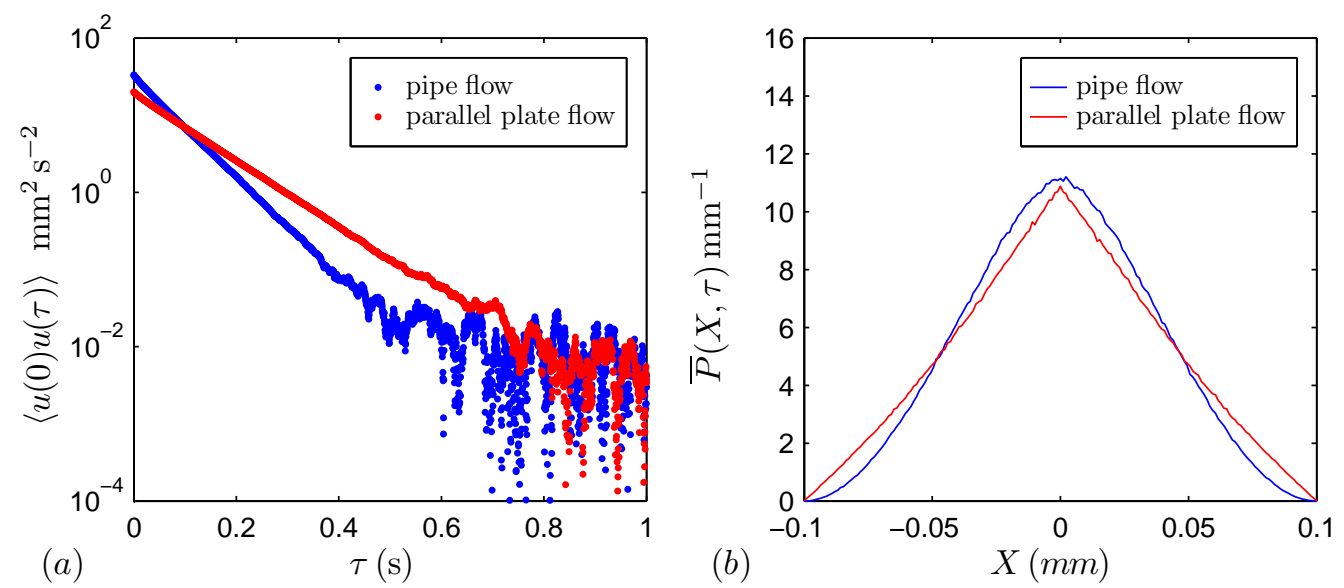

Figure 6.9: The simulated VACF (a) and the transverse propagators (b) for capillary flow (blue) and parallel plate flow (red). The tube velocity was taken to be $1.8 \mathrm{~mm} \mathrm{~s}^{-1}$ and the encoding time for the propagators was $0.5 \mathrm{~s}$. The slower decay of the VACF for parallel plate flow indicates stronger nonlocal dispersion terms at longer encoding times. The propagator shows that in the parallel plate flow a higher proportion or particles have moved $\pm 0.07 \mathrm{~mm}$. 

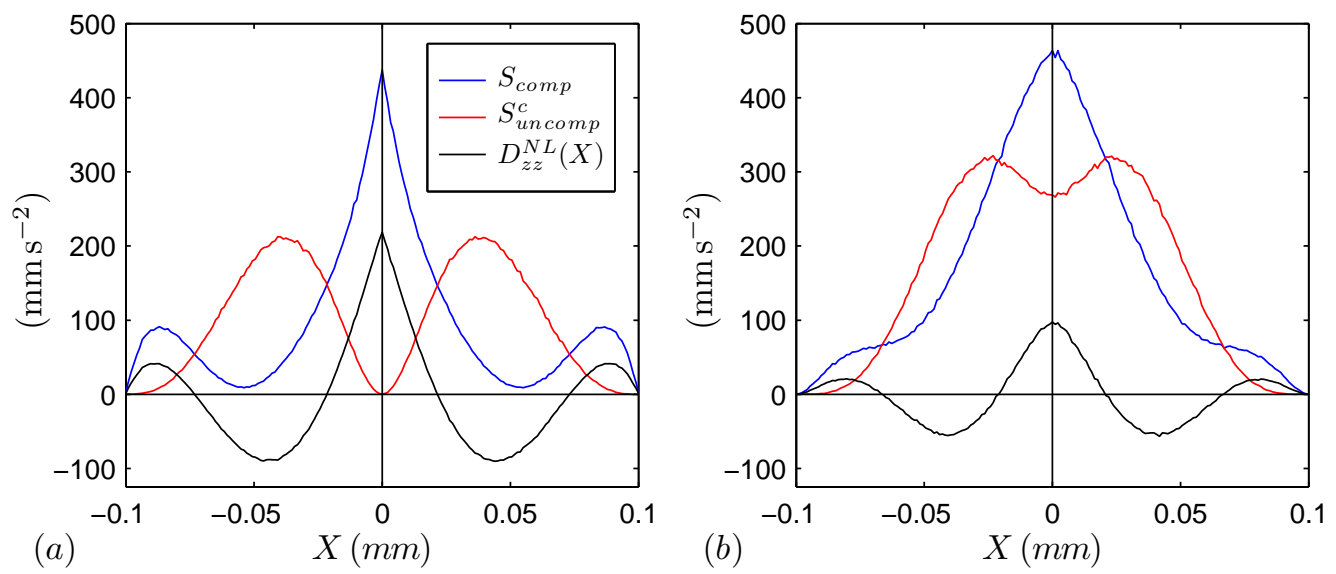

Figure 6.10: A simulation of the component $D_{z z}^{N L}(X, \tau)$ (black) for parallel plate flow (a) and capillary flow (b). In each graph the fit to the compensated (blue) and phase corrected uncompensated (red) data sets is shown, demonstrating the required superposition. In the case of parallel plate flow, the difference is greater allowing for better sensitivity

periment of a purely diffusion driven dispersion, flow in parallel plates would be a preferred system.

The comparison of the term $D_{z z}^{N L}(X, \tau)$ for capillary flow and parallel plate flow raises some interesting questions. Clearly the term for parallel plate flow shows a better defined structure. This is because the component $D_{z z}^{N L}(X, \tau)$ averages over any structure in the $Y$ and $Z$ displacements, and in the case of parallel plate flow, there is no correlation structure for $Y$ displacements. This suggests that if the component $D_{z z}^{N L}(X, \tau)$ for capillary flow could be resolved further into $Y$ and $Z$, more structure could be seen. As seen in figure 6.7 there is some structure when the longitudinal correlation is resolved in $Z$ only. Multidimensional encodings will be discussed in section 7.5 .5 and chapter 8

Perhaps the simplest nonlocal dispersion term is the component $D_{z z}^{N L}(Z, \tau)$ for pure laminar flow in a pipe. When this term is expressed in terms of $V_{z}$ we get a term independent of time, with a very simple expression 


$$
D_{z z}^{N L}\left(V_{z}\right)=\frac{1}{2 \bar{v}}\left(V_{z}-\bar{v}\right)^{2}
$$

This term obviously has some structure and would be relatively simple to measure. To minimise diffusive mixing a large radius sample could be used, resulting in good signal to noise. However the lack of mixing, absence of a velocity autocorrelation function and hence no time dependance somewhat diminishes the motivation for measurement.

\subsection{Taylor-Couette Flow}

Steady state flow in a Taylor-Couette cell may not seem a useful system for studying dispersion, if diffusive effects are small the flow is fully reversible and molecules tend not to move off stream lines. However, PGSENMR is sensitive to Cartesian components of the flow and displacement. The natural circular motion of the flow in a Couette cell provides ideal structure when the velocity is resolved into $x$ and $y$ components [88].

\subsubsection{Calculation of Nonlocal Dispersion}

A simple Couette cell, as shown in figure 6.11 provides an excellent system for testing the NMR measurements of $\mathbf{D}^{N L}(\mathbf{R}, \tau)$. Neglecting diffusion, the velocity field is simple to calculate and is a function of radial position $r$ only. The purely azimuthal velocity distribution in a Couette cell is given by, in cylindrical polar coordinates

$$
v(r)=\frac{V_{\text {max }} r_{i n}}{r_{\text {out }}^{2}-r_{\text {in }}^{2}}\left(\frac{r_{\text {out }}^{2}}{r}-r\right),
$$

where $V_{\max }$ is the fluid velocity at $r_{i n}$. The probability for the fluid element at a particular $r$ to move a distance $X$ in a time $\tau$ can be expressed as

$$
P(X, r, \tau)=\frac{2 r}{\pi\left(r_{\text {out }}^{2}-r_{\text {in }}^{2}\right) \sqrt{X_{\max }^{2}-X^{2}}}
$$




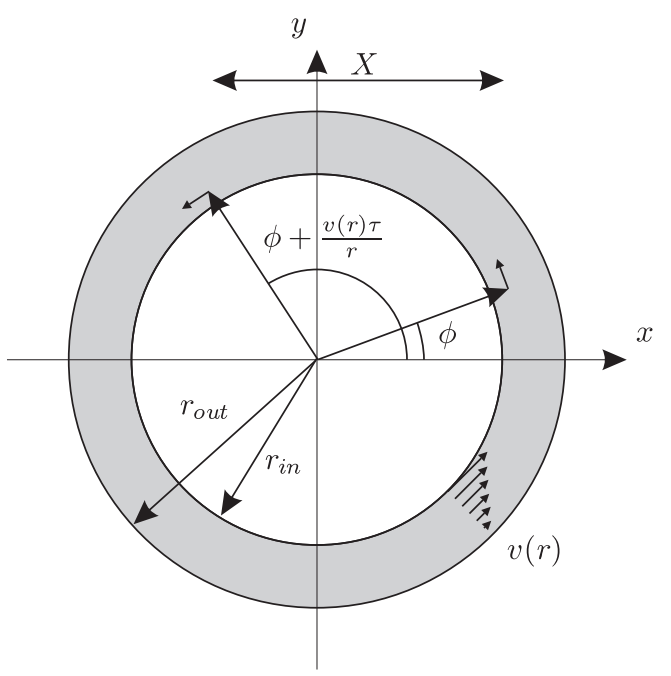

Figure 6.11: Relevant coordinate system and velocities for flow in a Couette cell where the inner cylinder is rotating. The velocity is a function of $r$ only. Measurement can be sensitive to either $X$ or $Y$ displacement and either $x$ or $y$ components of velocity.

where $X_{\max }=2 r \sin \frac{v(r) \tau}{2 r}$. This expression links the cylindrical polar coordinates of the velocity field and the Cartesian coordinates of the displacement measurement relevant to PGSE NMR experiments. The propagator can then be found by integrating this expression from $r_{\text {in }}$ to $r_{\text {out }}$. This integration is difficult to perform analytically or numerically due to the larger number of singularities when $X_{\max }^{2}=X^{2}$, and so the propagator is investigated statistically. The initial position of tracer particles are chosen at random within the fluid, their initial velocity and trajectory and hence final velocity being entirely determined by their initial position. A histogram of displacements can then be constructed, giving the propagator. Figure 6.12(a) shows the propagator at a range of encoding times. The propagator spreads out very quickly as the inner part of the cylinder makes its first rotation, the shape then develops slowly as it takes many rotations of the inner cylinder for some of the slower moving particles to 
complete a rotation. The particles near the wall are stationary and contribute to the poorly defined peak at $X=0$.

To calculate the nonlocal dispersion, we are sensitive to the components of velocity, either $v_{x}$ or $v_{y}$. These will depend not only on $r$ but also $\phi$ and as such we need to construct the propagator as a function of $r$ and $\phi$. In the absence of Taylor dispersion effects, the final position of any fluid element is known from its initial position. Thus the propagator can be written as a delta function.

$$
P(X, r, \phi, \tau)=\delta\left(r \cos \theta^{\prime}-r \cos \phi-X\right)
$$

where $\theta^{\prime}=\theta+\frac{v(r) \tau}{r}$. Assuming no motion in the $z$ direction there are 8 nonlocal dispersion components. Due to symmetry the three independent components that remain are

$$
\begin{aligned}
D_{x x}^{N L}(X, \tau) & = \\
& \int_{0}^{2 \pi} \int_{r_{\text {in }}}^{r_{\text {out }}} v(r) \sin \phi \delta\left(r \cos \phi^{\prime}-r \cos \phi-X\right) v(r) \sin \phi^{\prime} r d r d \phi, \\
D_{y y}^{N L}(X, \tau)= & \int_{0}^{2 \pi} \int_{r_{\text {in }}}^{r_{\text {out }}} v(r) \cos \phi \delta\left(r \cos \phi^{\prime}-r \cos \phi-X\right) v(r) \cos \phi^{\prime} r d r d \phi,
\end{aligned}
$$

and

$$
\begin{aligned}
D_{x y}^{N L}(X, \tau) & = \\
& \int_{0}^{2 \pi} \int_{r_{\text {in }}}^{r_{\text {out }}} v(r) \sin \phi \delta\left(r \cos \phi^{\prime}-r \cos \phi-X\right) v(r) \cos \phi^{\prime} r d r d \phi,
\end{aligned}
$$

where $\phi^{\prime}=\phi+\frac{v(r) \tau}{r}$ and $v(r)$ is given by equation 6.23 .

Equations 6.26 to 6.28 , like the expression for the Couette flow propagator, can be conveniently evaluated using a Monte-Carlo type integration. 
The initial distribution is chosen at random and this determines the final position and hence the nonlocal components can be generated statistically.

The three nonlocal dispersion components, shown in figure 6.12(b-d) show remarkably different structure and evolution characteristics. At $\tau=$ $0.25 T$, where $T$ is the time required for one complete rotation of the inner cylinder, the two terms $D_{x x}^{N L}(X, \tau)$ and $D_{y y}^{N L}(X, \tau)$ appear to show somewhat complementary structure. The term $D_{x y}^{N L}(X, \tau)$ is entirely positive, indicative of the rotational direction from positive $x$ to positive $y$ (see figure 6.11).

After $\tau>0.5 T$, sharp features begin to develop in the term $D_{y y}^{N L}(X, \tau)$, including a peak at $X=0$ for $\tau=1 T$. A similar peak is present in the propagator at all times, due to the particles on the wall of the outer cylinder and the particles that have completed one or more revolutions. In the nonlocal term however, contributions to this peak only come from particles that have completed a revolution, and hence will have a highly correlated velocity. Particles on the boundary will have no velocity and hence no correlation.

No such features appear in the terms $D_{x x}^{N L}(X, \tau)$ and $D_{x y}^{N L}(X, \tau)$, indeed these terms decay to zero relatively quickly while the term $D_{y y}^{N L}(X, \tau)$ has a well defined structure at infinite time.

\subsubsection{Experimental Results}

The Couette cell is constructed from NMR tubes such that, in accordance with figure $6.11, r_{\text {out }}=4.35 \mathrm{~mm}$ and $r_{\text {in }}=3.25 \mathrm{~mm}$ and the inner tube was rotated with a frequency of $4 \mathrm{~Hz}$. The rotation is provided by a RheoNMR stepper motor and drive shaft. The Rheo-NMR motor provides a TTL pulse every rotation, allowing the NMR pulse sequence to be synchronous with the rotation. To minimise diffusion effects the sample was 10,000 MW polydimethylsiloxane. In section 5.4.1 the need for a 32 step phase cycle was discussed. For complex flows crusher gradients around 


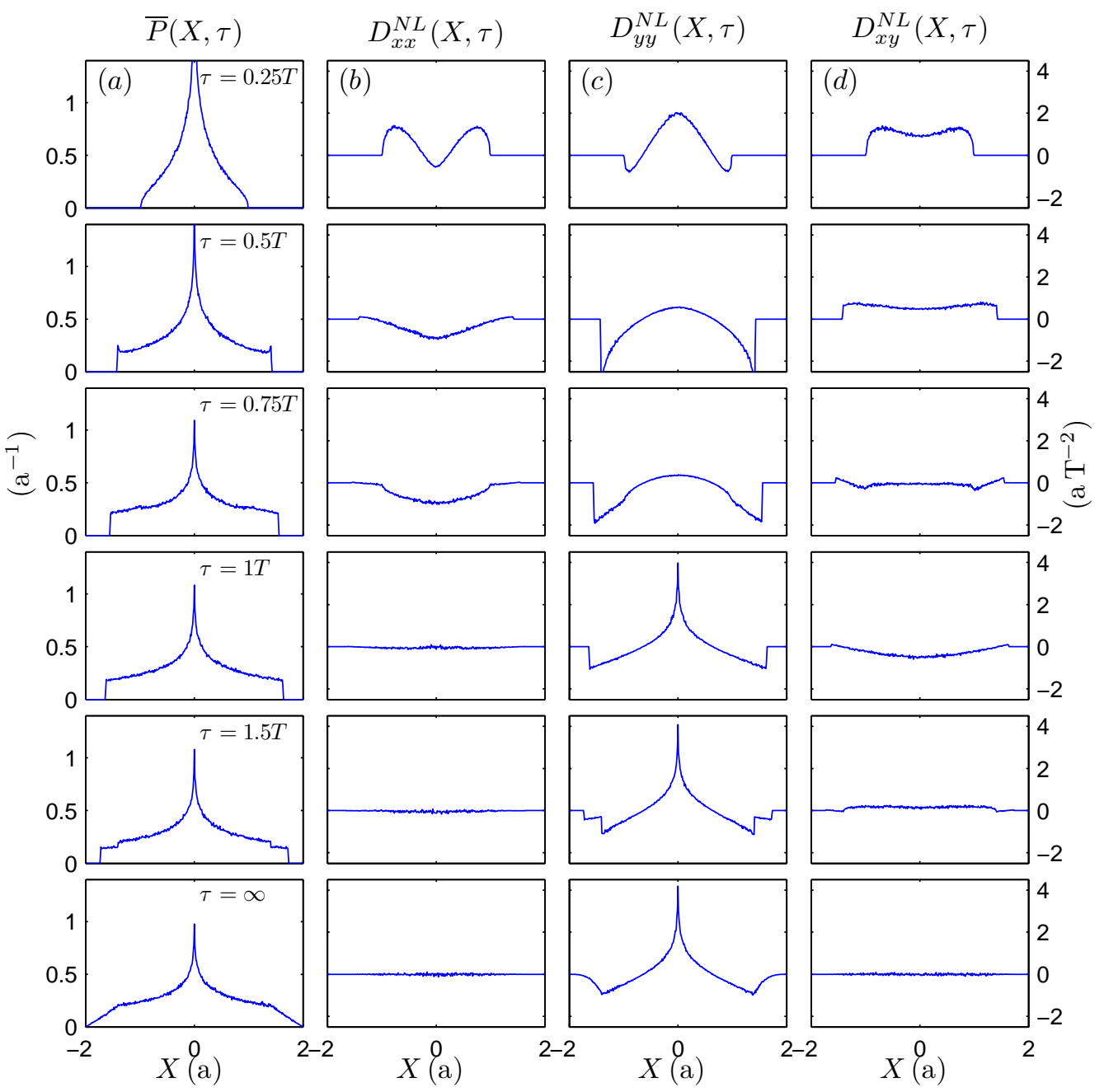

Figure 6.12: Simulated propagators (a) and nonlocal dispersion tensor components, $D_{x x}^{N L}(X, \tau)$ (b), $D_{y y}^{N L}(X, \tau)$ (c) and $D_{x y}^{N L}(X, \tau)$ (d) for Couette flow. The time $T$ is the time for one revolution of the inner cylinder. The greatest features and persistence are shown in the component $D_{y y}^{N L}(X, \tau)$.

the $180^{\circ}$ pulse were undesirable as this will give some unwanted encoding. In this Couette cell we have minimal motion along $z$, hence crusher pulses can be used in this direction. This reduces the phase cycle from 32 to 4 . The relatively large sample volume gives good signal-to-noise, a 


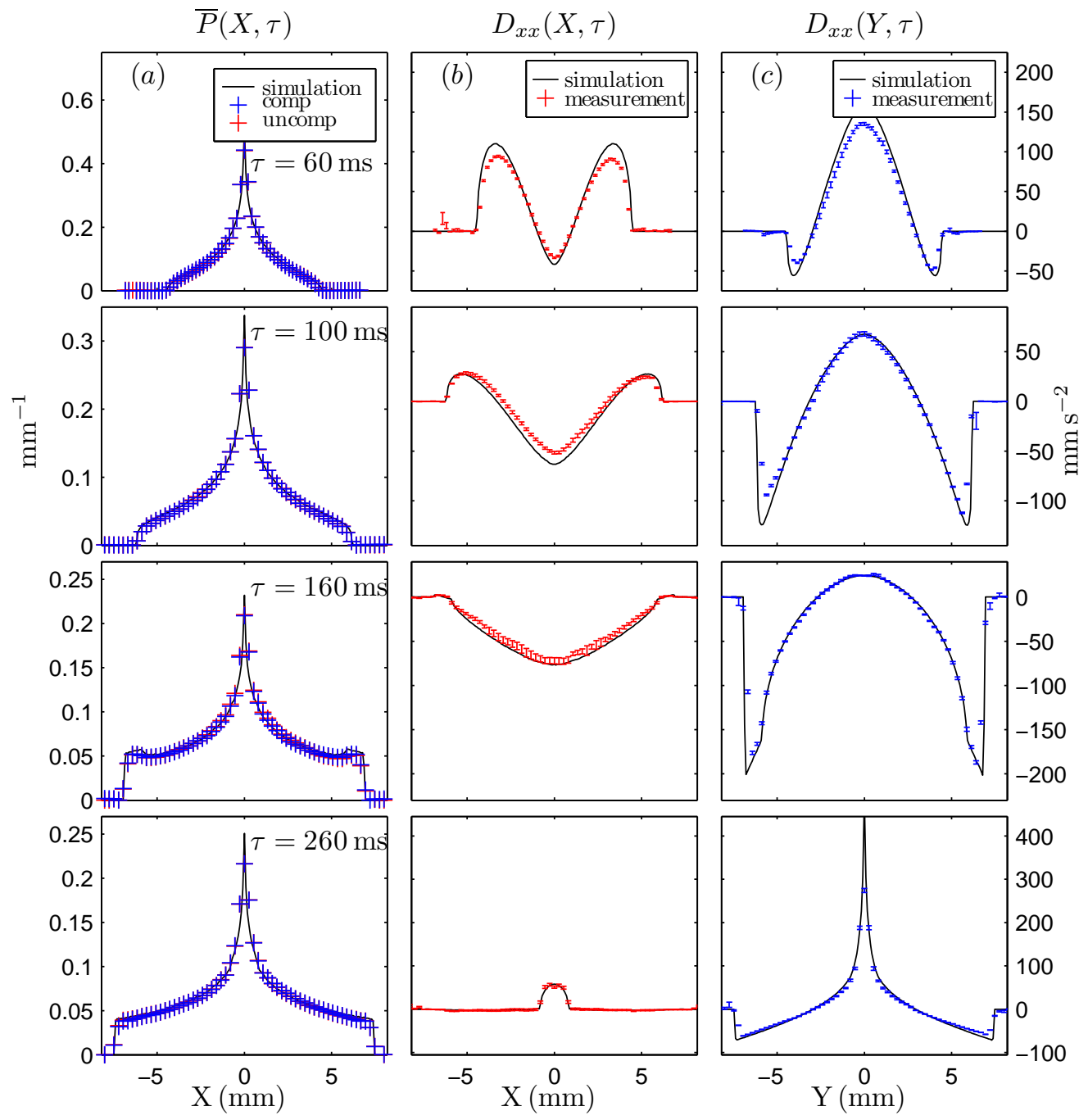

Figure 6.13: The simulated (solid black line) and experimentally measured (crosses) propagators (a) and nonlocal components $D_{x x}^{N L}(X, \tau)(\mathrm{b})$ and $D_{x x}^{N L}(Y, \tau)$ (c) for Couette flow. The agreement between simulation and measurement is excellent. The measured propagators in panel (a) are shown for each encoding time, from the uncompensated (red crosses) and compensated (blue crosses) experiments. In contrast to the pipe flow experiment, the self agreement is very good. 


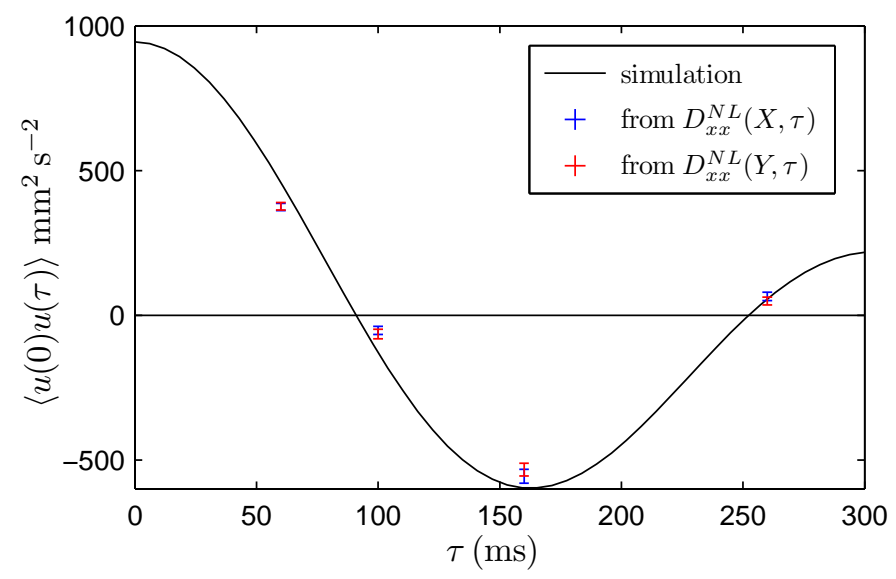

Figure 6.14: The simulated (solid black line) measured velocity autocorrelation function from the $D_{x x}^{N L}(X, \tau)$ component (red) and the $D_{x x}^{N L}(Y, \tau)$ component (blue). The VACF can be measured directly from the superposition of the compensated and uncompensated experiments with no displacement encoding.

condition we expect for flow in porous systems.

Four encoding times, $60 \mathrm{~ms}, 100 \mathrm{~ms}, 160 \mathrm{~ms}$ and $260 \mathrm{~ms}$ where chosen to measure the propagator and the components $D_{y y}^{N L}(X, \tau)$ and $D_{x x}^{N L}(X, \tau)$. There is no net flow and as such the $q_{u}$ dependent phase correction aspect of the superposition is not being tested. We have used 64 steps in the displacement encoding direction and 49 steps in the motion encoding direction. The results are shown in figure 6.13. In panel (a) the propagators are shown in comparison with the simulation. The agreement is very good. Also of note is the agreement between the measured propagators from the compensated and uncompensated pulse sequence. This is a good indication of the stability and reliability of the experiment. The low $-q$ fit in the motion encoding direction and subsequent superposition give the nonlocal dispersion coefficients in panels (b) and (c). The agreement is very good, many of the sharp features of the component $D_{y y}^{N L}(X, \tau)$ are accurately resolved. To further quantify the agreement, one would first inves- 
tigate any discrepancies in the VACF measurement shown in figure 6.14.

In addition to Couette flow providing an ideal model system for measuring nonlocal dispersion, the velocity autocorrelation function is one that one cannot be easily measured by previous techniques [16]. Figure 6.14 shows the measured VACF using the superposition method described in section 5.3.4. While the structure agrees very well there is a slight discrepancy in the amplitude of the correlation.

\subsection{Conclusions}

The experimental measurements on capillary flow did not provide a satisfactory verification of our NMR technique, this is due to the inherent difficulties of using a small sample and the need for a flow rate to be stable over a range of time scales. The nature of the superposition required for capillary flow is very demanding. In order to directly compare experimental results with a calculated, diffusion driven dispersive system, several suitable systems have been proposed: flow in a parallel plate system being the preferred method.

The comparison between the calculated and simulated nonlocal dispersion in capillary flow verifies our statistical techniques. The statistical generation of nonlocal components form an important part of the simulation suite described chapter 4 . Chapter 8 contains discussion on purely statistically generated nonlocal components.

The flow in a Couette cell provided an ideal system for verifying our experimental technique, with the exception of the $q_{u}$ dependent phase correction for the uncompensated experiment, all aspects are tested. This enables measurements to be performed on porous media, detailed in chapters 7 and 9, with a high degree of confidence. 


\section{Chapter 7}

\section{Nonlocal Dispersion in a Porous Medium}

\subsection{Introduction}

A random packing of monosized spheres, also known as a 'packed bead' or simply 'beadpack', provides a model system for porous media. The size of the pore space or the permeability can be tuned by the selection of readily available sphere sizes. A degree of heterogeneity can be specified by using bi-disperse or polydisperse spheres. Many 'real' porous media are difficult to fully characterise with NMR due to large internal gradients resulting from the susceptibility mismatch between the solid matrix and the liquids. A beadpack can be constructed with or without internal gradients depending on the choice of material for the beads.

The results presented in this chapter detail the first measurements of nonlocal dispersion components in a porous medium [158] and, in parallel a comparison was made with results from the simulation suite detailed in chapter 4 . This investigation, while extensive in its own right, covered a small range of short encoding times and was limited to a single Peclet number. 


\subsection{Experimental Method}

NMR experiments were performed on a Bruker AVANCE $400 \mathrm{MHz}$ spectrometer, with a microimaging gradient set capable of providing $1.45 \mathrm{~T} \mathrm{~m}^{-1}$. Distilled, degassed water was pumped (BVP-Z Ismatec) through a random bead pack of $500 \mu \mathrm{m}$ diameter latex monodisperse spheres (DUKE Scientific, Palo Alto, U.S.). A column of randomly packed beads was contained in a poly(ether ether ketone) (PEEK) cylinder of inner diameter $10 \mathrm{~mm}$. The beadpack was contained by plugs of porous plastic, outside the sensitive region of the $\mathrm{rf}$ coil, to a length of $50 \mathrm{~mm}$. A 'birdcage' resonator with a diameter of $15 \mathrm{~mm}$ was used. The porosity of the beadpack was $(37.5 \pm 0.5) \%$, as measured by NMR.

In order to dampen out high-frequency pulsations from the pump, a length of rubber tubing was used in series with the otherwise teflon tubing. Any remaining variation in the flow was not detrimental to the signal quality. The liquid was pumped at a tube velocity of around $10.2 \mathrm{~mm} \mathrm{~s}^{-1}$, giving a $\tau_{v}$ of $49.0 \mathrm{~ms}$, a Peclet number of approximately 1500 and a Reynolds number of approximately 3.3. Experiments reported here are performed at displacement encoding times of $10 \mathrm{~ms}, 21.5 \mathrm{~ms}$ and $46.3 \mathrm{~ms}$. The separation of each double-PGSE pair, $\Delta_{u}$, was $2 \mathrm{~ms}$, thus ensuring $\Delta_{u}<<\tau_{v}$. To reduce the delay between scans, caused by the need to allow for nearcomplete $T_{1}$ relaxation, the water was doped with $0.025 \mathrm{wt} \% \mathrm{GdCl}_{3}$ to give a $T_{1}$ of approximately $110 \mathrm{~ms}$.

Full details of the NMR pulse sequence, its implementation and subsequent post processing are given in sections 5.4.1 to 5.4.3. The time for one measurement is around 8 hours for transverse encodings and 12 hours for longitudinal. 


\subsection{Lattice Boltzmann Simulation}

Complementary lattice Boltzmann based simulations were performed in order to compare with the experimental results and to provide an efficient means of exploring a range of parameters which would be tedious to produce experimentally. Full details of the simulation suite are given in chapter 4 .

\subsection{Dimensionality of the Nonlocal Dispersion Tensor}

The statistically calculated nonlocal dispersion tensor allows for convenient examination of the non-zero and independent terms. Each encoding can be either parallel or transverse to the main flow, however, for a full survey we will treat the two orthogonal encodings independently. We use the subscripts $T, \perp$ and $\|$ to describe the two transverse directions and the longitudinal direction, respectively. Terms are equal when all of the transverse subscripts are swapped, that is

$$
D_{\perp \perp}^{N L}\left(X_{\perp}, \tau\right) \neq D_{\perp \perp}^{N L}\left(X_{\top}, \tau\right)
$$

but

$$
D_{\perp \perp}^{N L}\left(X_{\perp}, \tau\right)=D_{\top \top}^{N L}\left(X_{\top}, \tau\right)
$$

\section{Longitudinal Displacement Encoding}

When the displacement encoding is parallel to the flow, all the off-axis terms are zero, the non zero terms being $D_{\perp \perp}^{N L}\left(X_{\|}, \tau\right)$ and $D_{\|\|}^{N L}\left(X_{\|}, \tau\right)$. The latter component perhaps shows the most structure at long times, which is indicative of the persistence of the longitudinal velocity autocorrelation function. 


\section{Transverse Displacement Encoding}

The diagonal transverse displacement encodings show different behaviour, and one off-axis element is present, giving four independent components $D_{\perp \perp}^{N L}\left(X_{\perp}, \tau\right), D_{\top}^{N L}\left(X_{\perp}, \tau\right), D_{\|\|}^{N L}\left(X_{\perp}, \tau\right)$ and the off-axis component $D_{\perp \|}^{N L}\left(X_{\perp}, \tau\right)$. The off-axis component describes the correlations between transverse and longitudinal velocities. The correlation term itself is zero but when resolved by a transverse displacement aligned with the transverse velocity direction, as in the term $D_{\perp \|}^{N L}\left(X_{\perp}, \tau\right)$, some structure is seen. This is not seen when resolved in the other transverse direction $D_{\perp \|}^{N L}\left(X_{\mathrm{T}}, \tau\right)$, or in the longitudinal direction $D_{\perp \|}^{N L}\left(X_{\|}, \tau\right)$.

The three terms describing transverse correlations show different structure but when integrated over displacement will give the same results, namely the transverse velocity autocorrelation function $\left\langle u_{\perp}(0) u_{\perp}(\tau)\right\rangle$.

\subsection{Experiment and Simulation Results}

\subsubsection{Definition of Peclet Number}

The random beadpacks of the experiment and simulations differ. First the ratio of bead diameter to cylinder is 20 to 1 experimentally and 10 to 1 in simulations. Second, the porosity of the experimental beadpack was measured as $(37.5 \pm 0.5) \%$ whereas the simulated beadpack has a porosity of around 0.71 . This has implications for the matching of Peclet number and the choice of encoding time.

The common experimental definition of Peclet number is given as [14]

$$
\mathrm{Pe}=\left(\frac{\phi}{1-\phi}\right) \frac{\langle v\rangle d_{\text {bead }}}{D_{0}}
$$

where $\phi$ is the porosity, $D_{0}$ is the self diffusion coefficient and $\langle v\rangle$ is the interstitial velocity. This includes a definition of the 'characteristic length' as $l=(\phi /(1-\phi)) d_{\text {bead }}$. Because of the difference in porosity between the 
experimental and simulated beadpacks there is a marked difference in the characteristic length, when expressed as a fraction of the bead diameter. For the experimental beadpack we have $l_{\text {exp }}=0.60 d_{\text {bead }}$ and in simulation, $l_{\text {sim }}=2.4 d_{\text {bead }}$. Note that a characteristic time $\tau_{v}$ may be defined, corresponding to the time to flow a bead diameter,

$$
\tau_{v}=\frac{d_{\text {bead }}}{\langle v\rangle} .
$$

In this case, to enable meaningful comparisons between experiment and simulation it may be more natural to look at encoding times expressed in terms of the time $\tau_{l}$ to flow a characteristic length. For completeness, both comparisons will be made.

\subsubsection{Propagator Measurement}

The six independent components and propagators were measured at three displacement encoding times $10 \mathrm{~ms}, 21.5 \mathrm{~ms}$ and $46.3 \mathrm{~ms}$, corresponding to the approximate logarithmic distribution of $0.20 \tau_{v}, 0.44 \tau_{v}$ and $0.94 \tau_{v}$. In terms of the time to flow a characteristic length the encoding times are $0.32 \tau_{l}, 0.70 \tau_{l}$ and $1.5 \tau_{l}$. It is clear that the mixing is well within the preasymptotic regime where it is expected that most structure will be shown in the nonlocal dispersion tensor [18]. Once we have these six measurements we can begin to infer characteristics of the flow.

It is clear from the measured and simulated longitudinal propagators in figure 7.1 that there are significant differences between the two systems. Note the use of a displacement axis for the transverse direction and a velocity axis for the longitudinal. The use of the velocity axis makes for easier comparison over widely different encoding times where longitudinal displacements may substantially differ. The simulations show a large number of particles at slow, or zero flow and shows little mixing over a time of $\tau=\tau_{v}$. In contrast the measured data show a Gaussian tendency at $\tau=\tau_{v}$. The simulated propagators when encoding times are expressed as 

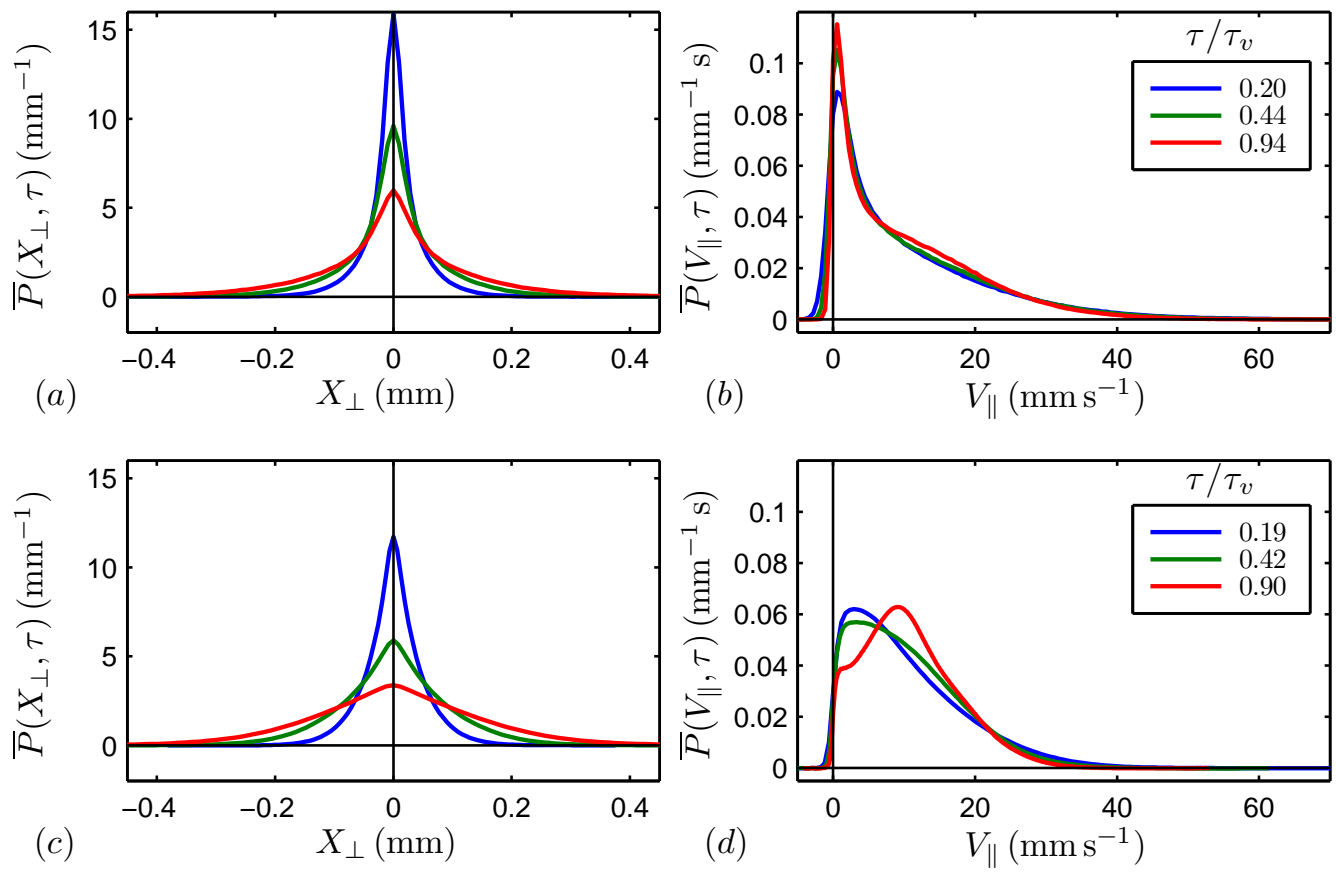

Figure 7.1: The simulated $(a, b)$ and measured $(c, d)$ propagators. The simulated longitudinal propagators (b) clearly show a larger proportion of slower moving particles than the measured (d). The greater mixing shown in the experimental measurements over simulation highlights the difference between the two systems.

a function on $\tau_{l}$ are more favourable, figure 7.2, and show the distribution moving towards Gaussian for times $\sim 1.5 \tau_{l}$. Nevertheless, the simulations show a large subset of particles with zero flow. Other groups [22] have had success in using a lattice Boltzmann simulation on a lattice generated directly from a high resolution MRI image of a real bead-pack, and subsequently using a monte-carlo simulation to produce agreement with measured propagators. It is not the goal of this work to be able to fully predict NMR results with a simulation technique, merely to develop two independent methods for inspecting the nonlocal dispersion tensor. Hence the preferred method of independently generating the simulated beadpack. 


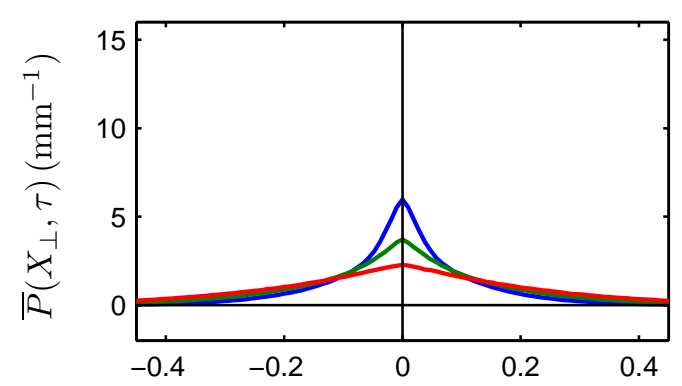

(a)

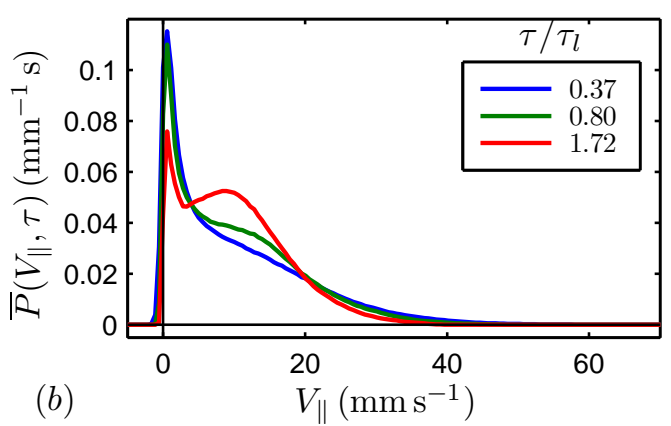

$(b)$

Figure 7.2: Simulated propagators with encoding times in terms of $\tau_{l}$. When expressed in fractions of the time to flow a characteristic length, the experimental propagators in figure $7.1(\mathrm{c}, \mathrm{d})$ have encoding times of a similar fraction.

\subsection{3 $\quad \mathbf{D}^{N L}$ Components from Simulation and Experiments}

The simulated and measured nonlocal dispersion components clearly show a good agreement for encoding times matched via the time to flow a bead diameter (figures 7.3 and 7.4) in the cases when transverse correlations are expressed. The agreement between the terms describing longitudinal correlations is acceptable. Interestingly the greatest difference in structure is highlighted by the cross-correlation term, $D_{\perp \|}^{N L}\left(X_{\perp}, \tau\right)$. Here the strong measured correlation describing particles moving a small distance in $-X_{\perp}$, is not present in simulation.

While the agreement between the simulated and experimental propagators was best with encoding times expressed as $\tau_{l}$, the agreement of the nonlocal dispersion components are mixed (figures 7.4 and 7.5). The agreement between components describing longitudinal velocity correlations is very good, but the transverse terms are poor. In this case the measured structure of the cross-correlation term $D_{\perp \|}^{N L}\left(X_{\perp}, \tau\right)$ is more clearly seen in the simulations, albeit with a different magnitude.

The clear differences are not seen as a failing of either method but perhaps shows the capacity of nonlocal dispersion measurements to highlight 

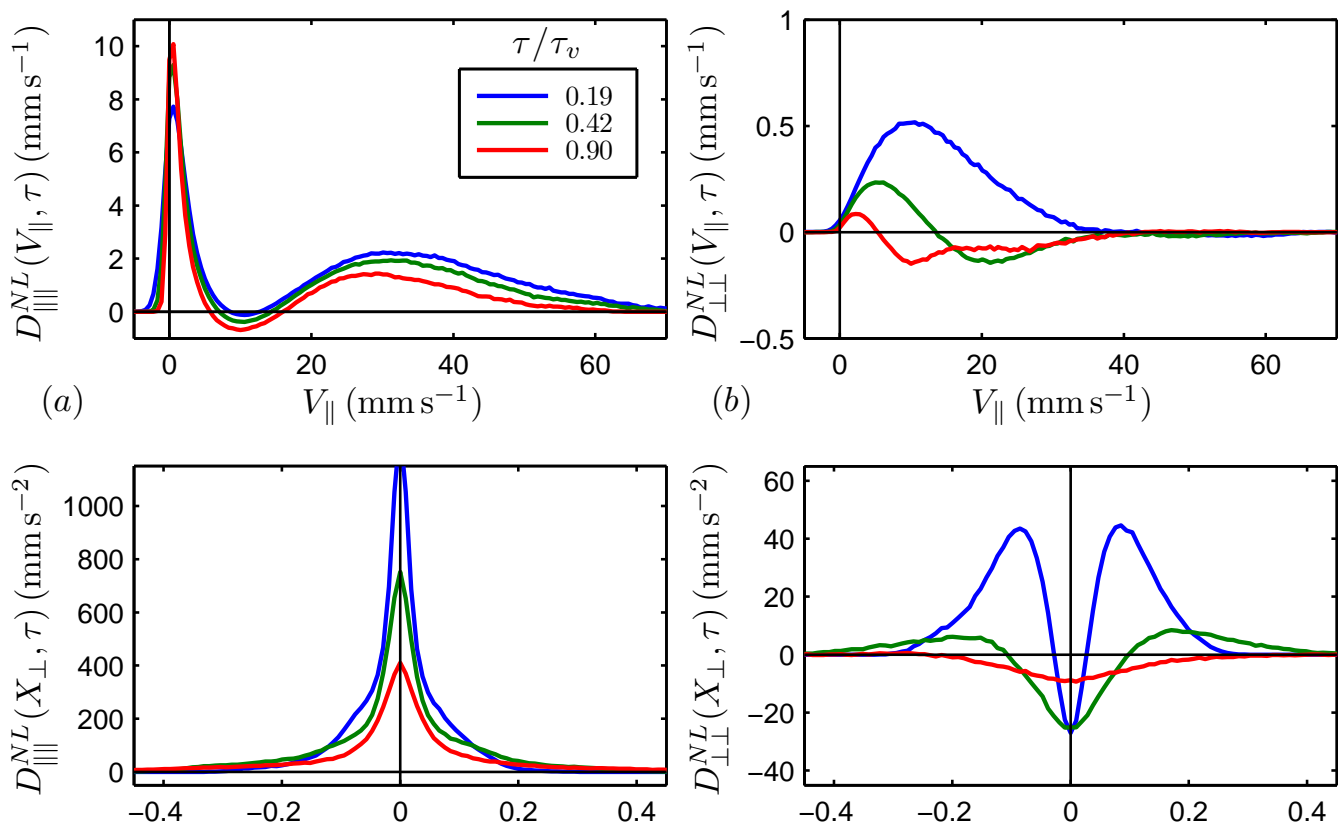

(c)
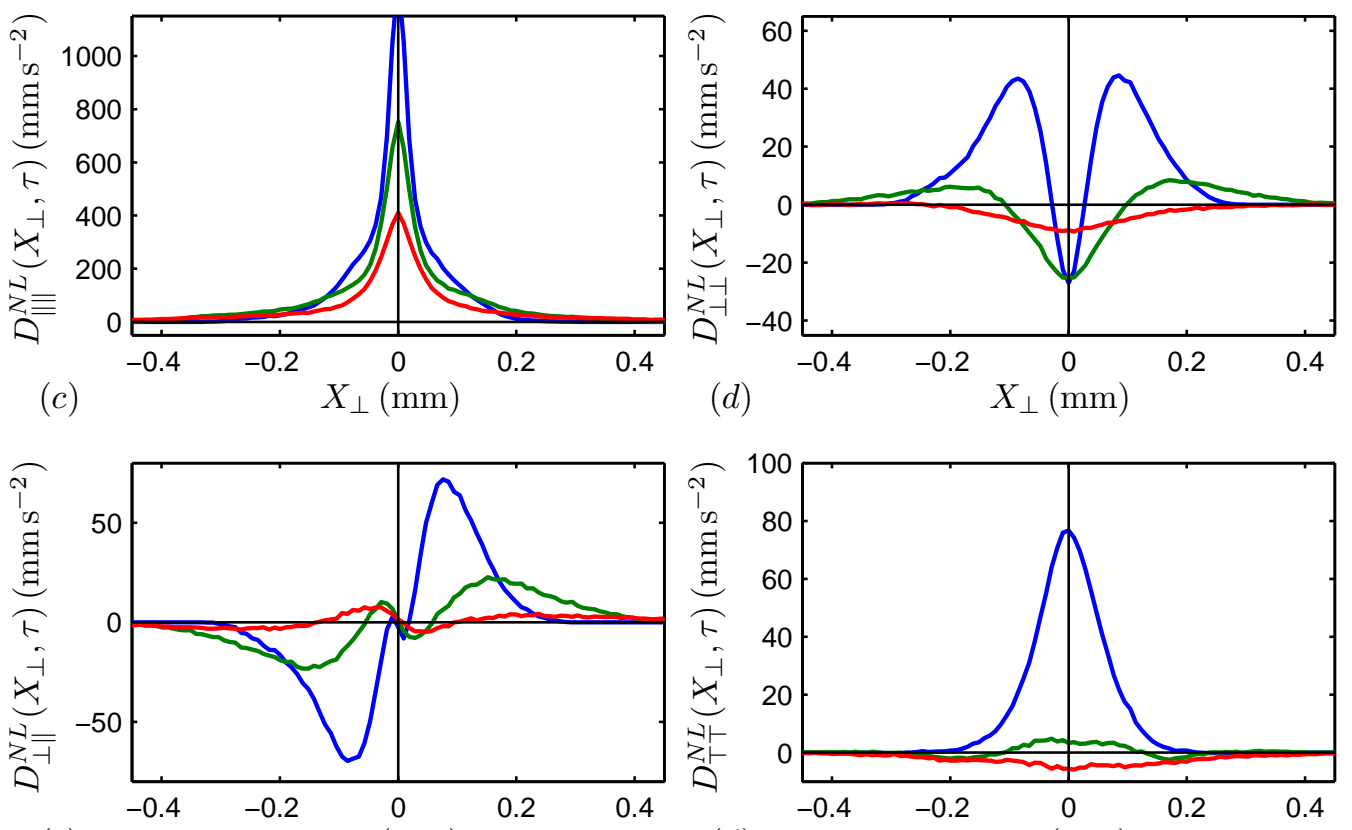

(e) $X_{\perp}(\mathrm{mm})$

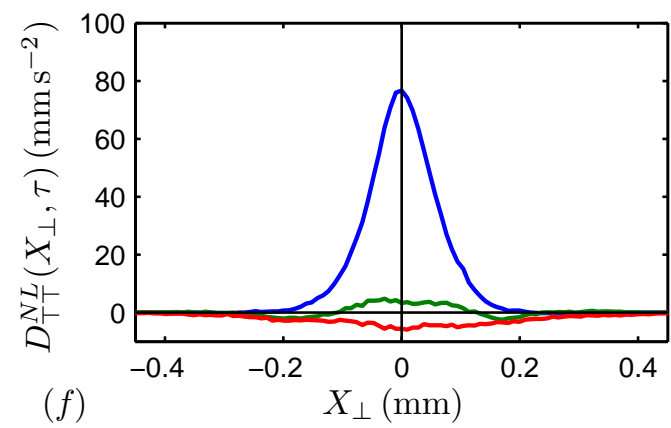

Figure 7.3: The six independent nonlocal dispersion tensor components for flow in a porous medium, simulated. See text for discussion. The components in the direction of the main flow are plotted as average velocity, rather than displacement.

subtle differences in the behaviour of systems where particles have moved over short times or short distances. Further discussions will involve encoding times expressed in the time to flow one bead diameter, $\tau_{v}$. 

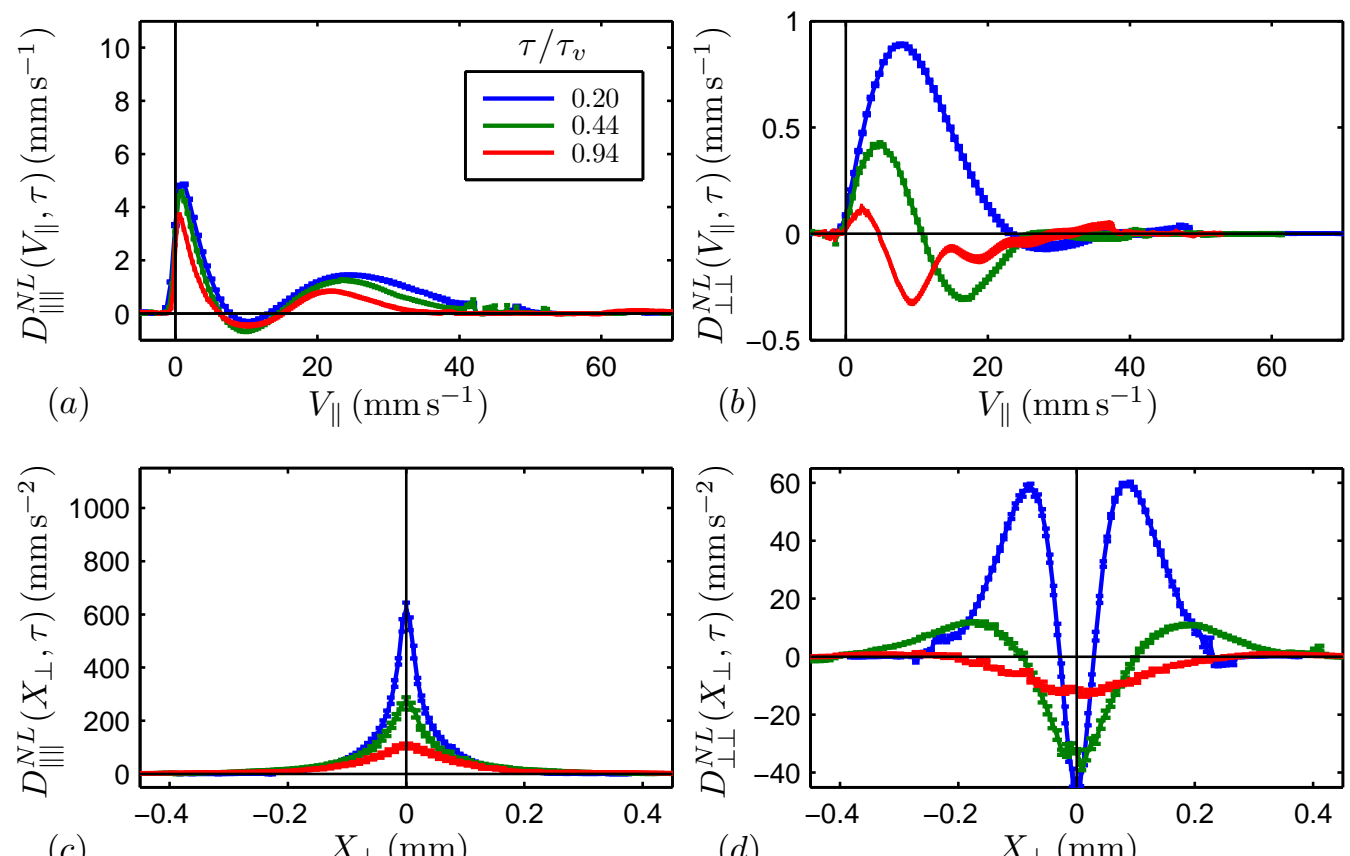

(c)

$(d)$

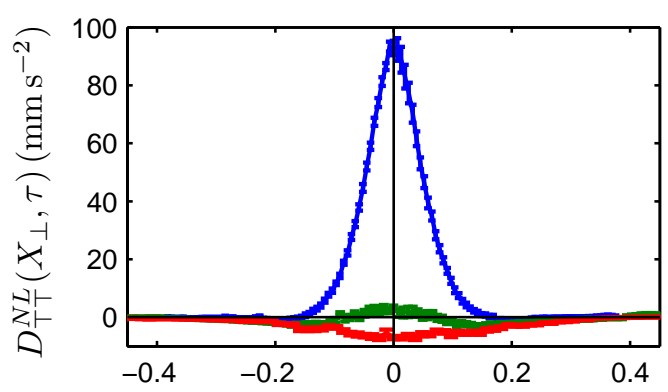

(e)

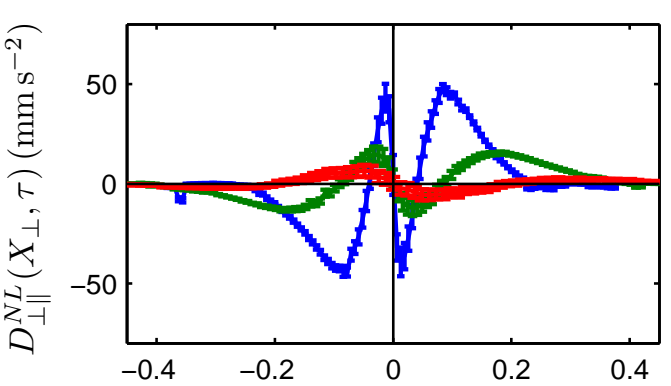

$(f)$

$$
X_{\perp}(\mathrm{mm})
$$

Figure 7.4: The six independent nonlocal dispersion tensor components for flow in a porous medium, measured by NMR. See text for discussion. The components in the direction of the main flow are plotted as average velocity, rather than displacement. The error bars show the estimate of the error in the fit to $q^{2}$.

\subsubsection{Analysis of Longitudinal Displacement Encodings}

The nonlocal dispersion tensor contains correlation information that is suppressed in the VACF. Considering the longitudinal VACF $\left\langle u_{\|}(0) u_{\|}(\tau)\right\rangle$, 

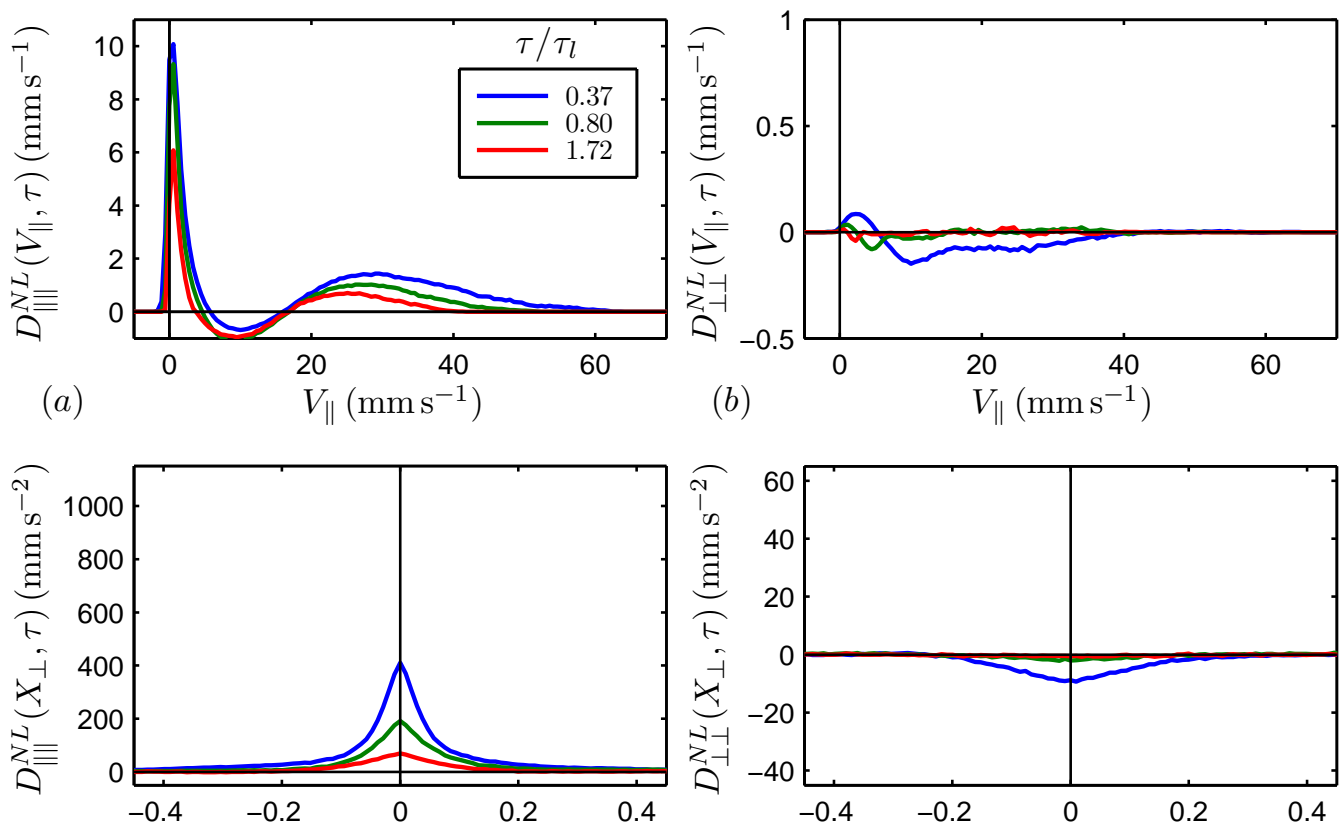

(c)
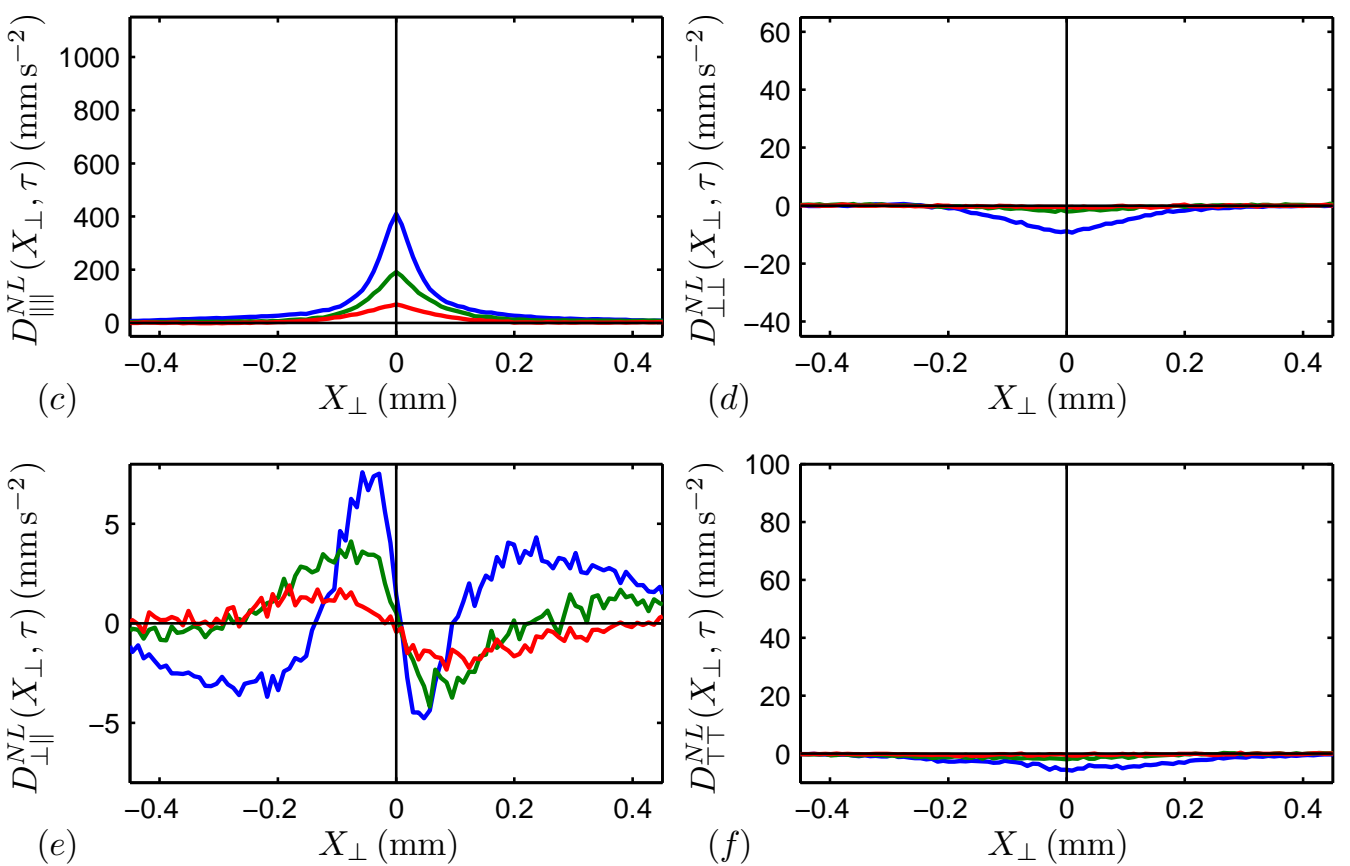

Figure 7.5: The six independent nonlocal dispersion tensor components for flow in a porous medium, simulated at times to match the experimental measurement times when expressed as $\tau_{l}$. With the exception of the component $D_{\perp \|}^{N L}\left(X_{\perp}, \tau\right)$ the components are plotted on the same scale as figure 7.4. See text for discussion. The components in the direction of the main flow are plotted as average velocity, rather than displacement.

we see that in figure 7.4(c) and (a) we have two ways of describing the correlations, first displaced by $X_{\perp}$ and second by $X_{\|}$, or as shown, $V_{\|}$. The 
component $D_{\|\| \|}^{N L}\left(V_{\|}, \tau\right)$ shows three distinct regions, a correlation, anticorrelation and a further correlation. That at lowest $V_{\|}$highlights the slow and stationary part as having a very highly correlated $u_{\|}$. Since these particles are moving slowly with respect to the mean flow, they will have a large, negative, $u_{\|}$which will be relatively unchanged at short $\tau$. The unusual feature of this peak is that while this peak will contribute quite strongly to the VACF, indicating a high degree of correlation, the flow in the laboratory frame is very small. The second correlation peak arises from particles that have moved a considerable distance in the encoding time, and yet retained a high degree of correlation. While the measured longitudinal propagator shown in figure 7.1(d) does not show a significant fraction of particles moving that distance, the nonlocal dispersion component $D_{\|\| \|}^{N L}\left(V_{\|}, \tau\right)$ clearly reveals this and shows its contribution to the VACF.

It is much more efficient to investigate the full position and time dependance in simulation. As an example the nonlocal component $D_{\|\| \|}^{N L}\left(V_{\|}, \tau\right)$ is shown for times up to $20 \tau_{v}$ in figure 7.6. The time evolution of the two correlation regions and the anticorrelation region can be seen. Furthermore, these three regions, shown for clarity in figure 7.7 can be independently integrated and monitored as a function of $\tau$. Figure 7.8 shows the contribution from each region to the VACF. It is interesting to note that the two correlation regions show different characteristic times, the faster correlation decay of the second region is perhaps due to the mechanical mixing of the flowing particles. In contrast the particles in the 1st region, which are stationary or moving slowly, show a longer timescale, perhaps dominated by Taylor or hold-up dispersion. The mean position in dimensionless units of the three regions are also shown in figure 7.9 to a longer encoding time. The maximum contribution from the anticorrelation occurs at around $2.5 \tau_{v}$. 


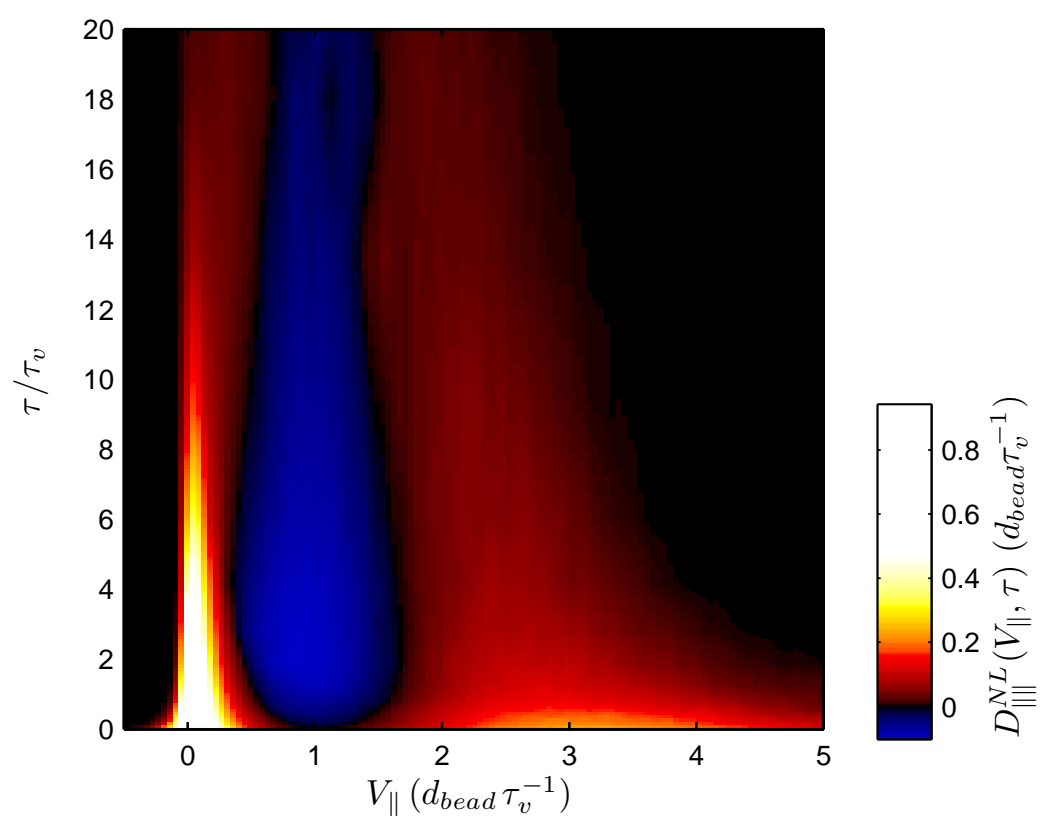

Figure 7.6: The purely longitudinal nonlocal dispersion component $D_{\|\| \|}^{N L}\left(V_{\|}, \tau\right)$ from simulation shown as a function of $\tau$ also. The colour map has been saturated to show the structure. Lighter colours correspond to a positive correlation, darker colours correspond to an anticorrelation.

\subsubsection{Analysis of Transverse Displacement Encodings}

In order to illustrate the extra information that resides in the nonlocal dispersion tensor, we consider the two tensor components $D_{\perp \perp}^{N L}\left(X_{\perp}, \tau\right)$ and $D_{\perp \perp}^{N L}\left(X_{\|}, \tau\right)$. These elements in fact describe exactly the same temporal correlation function when integrated over displacement. But extracted, they reveal spatio-temporal characteristics capable of providing physical insight. To illustrate that point we show in figure 7.10 our inference regarding the behaviour of the particles in $X_{\perp}$ and $Z_{\|}$that contribute to that correlation. In figure 7.10, the term $D_{\perp \perp}^{N L}\left(X_{\perp}, \tau\right)$ at an encoding time of $0.44 \tau_{v}$ shows a strong anticorrelation for particles that have moved not very far, if at all, in the $X_{\perp}$ direction (region $A$ ). The region of $D_{\perp \perp}^{N L}\left(X_{\|}, \tau\right)$ 


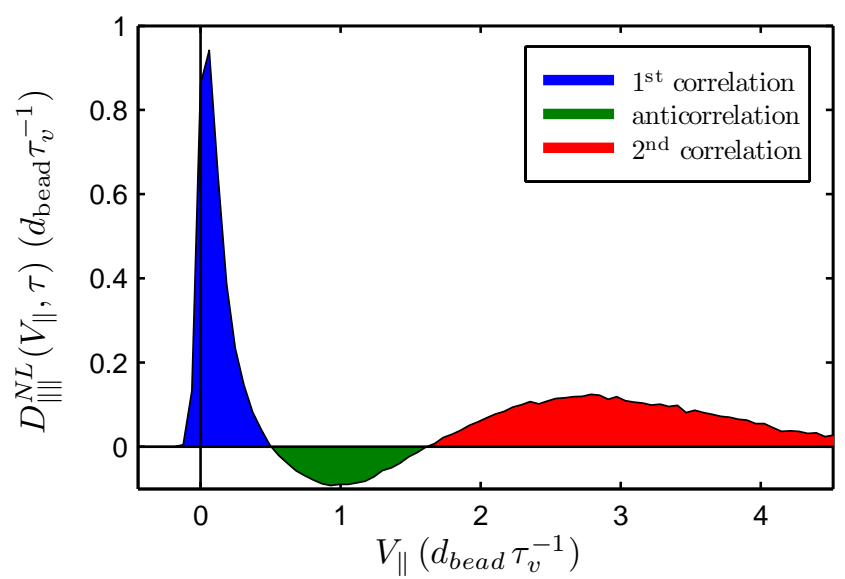

Figure 7.7: The purely longitudinal nonlocal dispersion component shows three distinct regions, in this case the mixing time is equal to $0.90 \tau_{v}$. The contribution of each region to the VACF can be investigated as a function of $\tau$. The colours of the three regions correspond to the legend of figures 7.8 and 7.9.

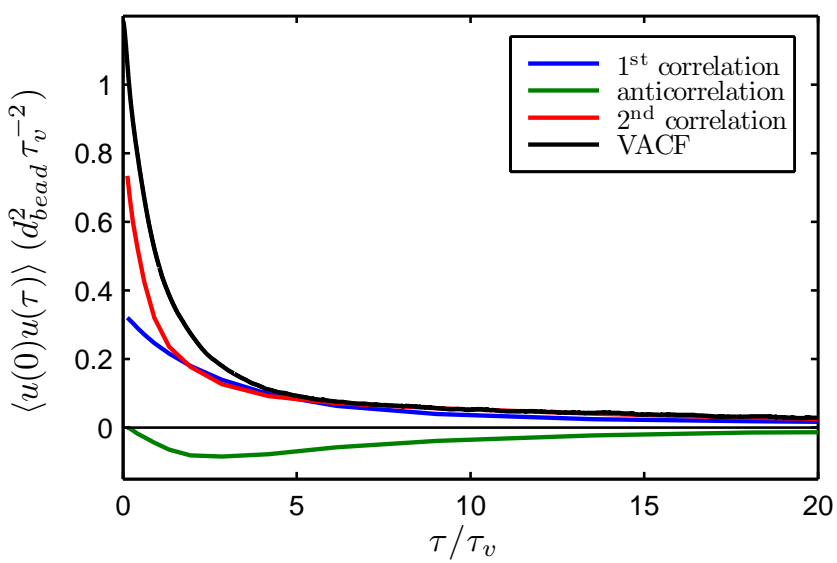

Figure 7.8: The contributions from the three regions as defined in figure 7.7. At very short $\tau$, i.e. $\tau<0.1 \tau_{v}$, there is no anticorrelation and hence no distinct regions exist. 


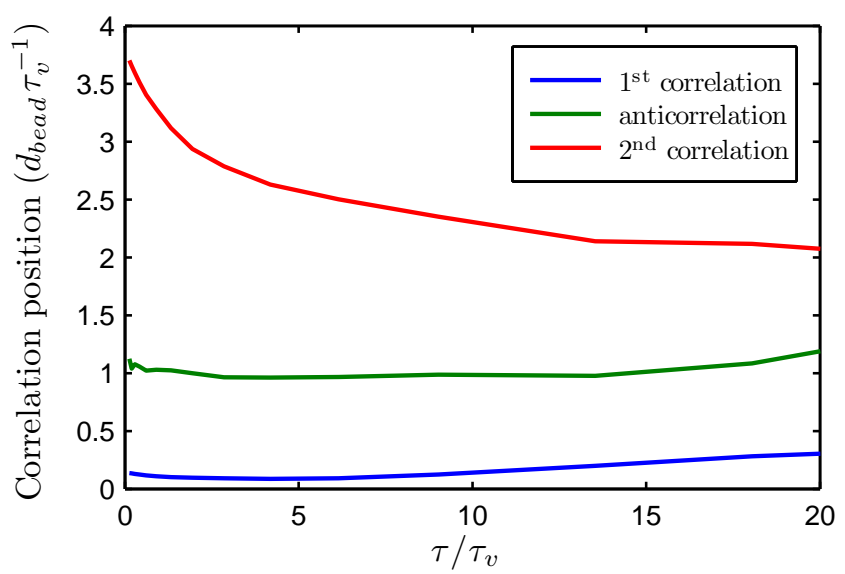

Figure 7.9: The movement of the mean of each of the three regions as defined in figure 7.7. The anticorrelation lobe is relatively unchanged but the 1st correlation region is slowly populated with slightly faster moving particles as $\tau$ increases, while the 2 nd correlation is rapidly populated with slower moving particles.

labelled $D$ shows an anticorrelation for particles that have moved a greater distance than the average. We might surmise that the subset of particles that are anticorrelated correspond to particles similar to that labelled $i$ that move round a bead as shown in figure 7.10. The particles with a large correlation have moved a short distance in $X_{\|}$. In the case of region $C$ we might guess that these particles are on a slow moving stream-line $j$, and as a result have moved in the same direction as the $u_{\perp}$ component of velocity, region $B$.

Of course, the schematic of figure 7.10 is merely a speculation. To confirm that inference, a simultaneous encoding in $X_{\perp}$ and $X_{\|}$would be necessary, thus providing a two-dimensional nonlocal dispersion tensor. The extra displacement dimension can easily be added to the NMR experiment with time as the only cost. In simulation the 'binning' as discussed in section 7.3 can include a second dimension. A large number of tracer particles, $\sim 10^{7}$, is required in order to produce statistically reliable es- 

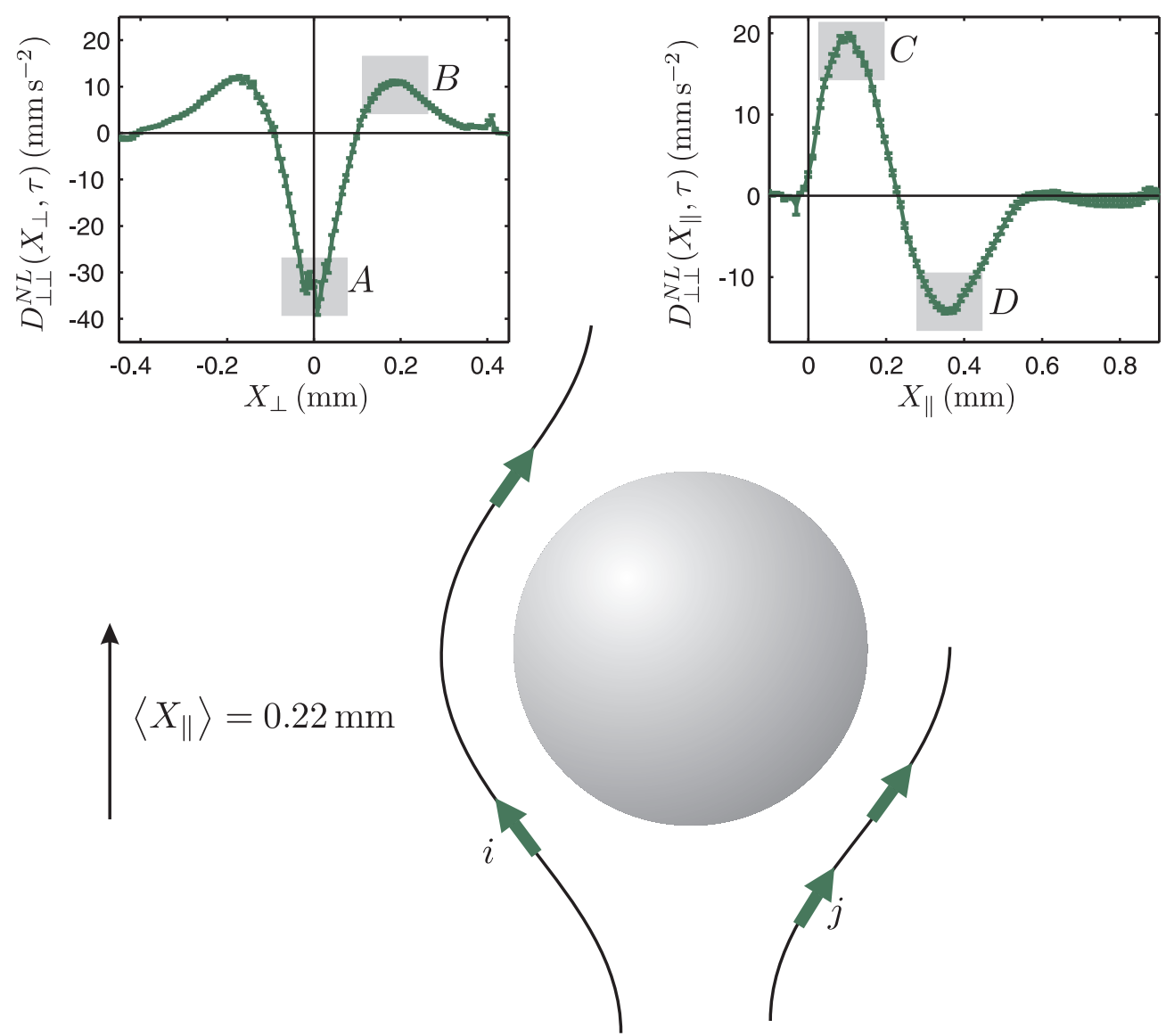

Figure 7.10: Inferring the behaviour of particles moving round a bead with experimental measurements. Consider the $D_{\perp \perp}^{N L}\left(X_{\perp}, \tau\right)$ and $D_{\perp \perp}^{N L}\left(X_{\|}, \tau\right)$ at an encoding time such that the mean displacement is approximately half a bead diameter. A guess is made that particles similar to $i$ make up the parts of the graphs $A$ and $D$, whereas the correlations shown as $B$ and $C$ are from particles similar to $j$. See text for complete discussion.

timates. Indeed the experimental data (figure 7.11) shows three distinct regions, the particles that move simultaneously in $X_{\perp}$ and $X_{\|}$showing a strong correlation in $u_{\perp}$. The particles that show a strong anticorrelation occupy a broad region and are clearly not limited to those which have moved a small $X_{\perp}$. This two-dimensional measurement is consistent with 
our interpretation but perhaps highlights a little naivety. In the component $D_{\perp \perp}^{N L}\left(X_{\perp}, \tau\right)$ the strong correlation of the slower diagonally moving particles is suppressed and is not as clear as in the two-dimensional encoding.

This two-dimensional measurement suggests that it is perhaps difficult to ascribe importance to the features in a single nonlocal measurement. The sharp distinction between correlation and anticorrelation lobes shown in $D_{\perp \perp}^{N L}\left(X_{\perp}, \tau\right)$ and $D_{\perp \perp}^{N L}\left(V_{\|}, \tau\right)$ are the result of the integral of the two-dimensional case. This would cast doubt on our analysis of the three regions in the purely longitudinal nonlocal component $D_{\|\| \|}^{N L}\left(V_{\|}, \tau\right)$ in section 7.5.4. However the other nonlocal component describing the longitudinal correlation $D_{\|\|}^{N L}\left(X_{\perp}, \tau\right)$ shows very little structure. As will be seen in chapter 8 , there is little structure when this correlation is resolved in three dimensions.

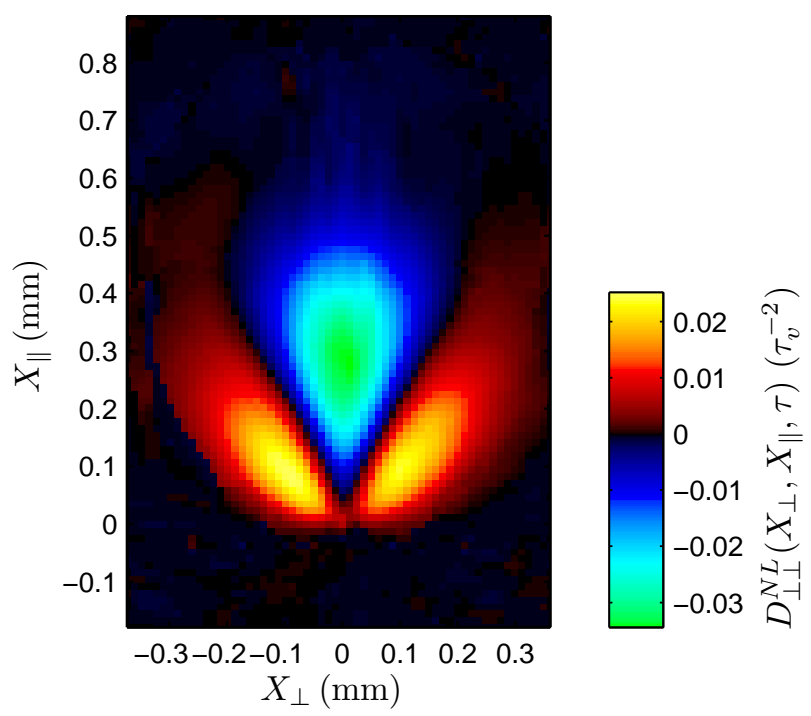

Figure 7.11: The experimental two dimensional nonlocal dispersion for $\tau=$ $0.44 \tau_{v}$ shows that there is a strong correlation (lighter colours) for particles that move simultaneously in $X_{\perp}$ and $X_{\|}$. The anticorrelation (darker colours) can be seen for a small range of $X_{\perp}$ at $X_{\|}$displacements around the average. 


\subsubsection{Other Tensors}

The difference superposition and fit to $q^{2}$ in equation 5.9 is designed to extract the correlations that yield the nonlocal dispersion tensor. An additive superposition and fit to $q^{2}$ will give a term that can be interpreted as the second moment of $u$ resolved by displacement. This term is potentially interesting as it gives some structure to the second moment, and because this structure shows temporal information.

Additionally, a fit in $q$ will give the first moment of $u$ resolved by displacement. This term is interesting since the first moment is, by definition, zero but with displacement resolution we have some displacement and temporal structure. The extra tensors do not contain any information correlating velocities in space and time and the superposition from the two pulse sequences used here is not required to measure them. We note these tensors are available because the encoding necessary for nonlocal dispersion allows their extraction.

In experiments where the motion encoding is parallel to the main flow, the necessary phase correction due to $\langle v\rangle$ can be omitted, thus giving tensors not in $u$ but $v$. It is assumed that tensors in $u$ give more structure, since the distinction between velocities above and below the mean are clear, but nevertheless, these quantities are available for examination. In particular nonlocal dispersion components describing the correlation of $v$ velocities can be obtain. These are denoted by $D_{\alpha \beta}^{N L v}\left(X_{\gamma}, \tau\right)$ and are shown in figure 7.14. Of course these terms only differ from the conventional nonlocal components when one or both of the chosen velocity encodings is in the direction of the main flow.

Figures 7.12 to 7.16 show the complete set of extra tensors available from the same experiments that produced the six nonlocal component shown in figure 7.4. Where possible the panels are arranged so that similar encodings are assigned the same letter. For example panel (a) in figure 7.4 and figures 7.12 to 7.16 all show components with the velocity and displacement encoding parallel to the main flow. This results in some uncon- 


\begin{tabular}{|c|ll|l|}
\hline Nonlocal dispersion Tensor & $D_{\|\|}^{N L}\left(X_{\|}, \tau\right)$ & $D_{\perp \perp}^{N L}\left(X_{\|}, \tau\right)$, & Figure 7.4 \\
& $D_{\|\|}^{N L}\left(X_{\perp}, \tau\right)$ & $D_{\perp \perp}^{N L}\left(X_{\perp}, \tau\right)$, & \\
& $D_{\perp \|}^{N L}\left(X_{\perp}, \tau\right)$ & $D_{\top}^{N L}\left(X_{\perp}, \tau\right)$ & \\
\hline First moment of velocity & $\left\langle u_{\|} \bar{P}\left(X_{\|}, \tau\right)\right\rangle$ & & Figure 7.12 \\
& $\left\langle u_{\|} \bar{P}\left(X_{\perp}, \tau\right)\right\rangle$ & $\left\langle u_{\perp} \bar{P}\left(X_{\perp}, \tau\right)\right\rangle$ & \\
\hline Second moment of velocity & $\left\langle u_{\|}^{2} \bar{P}\left(X_{\|}, \tau\right)\right\rangle$ & $\left\langle u_{\perp}^{2} \bar{P}\left(X_{\|}, \tau\right)\right\rangle$ & Figure 7.13 \\
& $\left\langle u_{\|}^{2} \bar{P}\left(X_{\perp}, \tau\right)\right\rangle$ & $\left\langle u_{\perp}^{2} \bar{P}\left(X_{\perp}, \tau\right)\right\rangle$ & \\
& & $\left\langle u_{\top}^{2} \bar{P}\left(X_{\perp}, \tau\right)\right\rangle$ & \\
\hline Nonlocal dispersion in $v$ & $D_{\|\|}^{N L v}\left(X_{\|}, \tau\right)$ & & Figure 7.14 \\
& $D_{\|\|}^{N L v}\left(X_{\perp}, \tau\right)$ & & \\
& $D_{\perp \|}^{N L v}\left(X_{\perp}, \tau\right)$ & & \\
\hline First moment in $v$ & $\left\langle v_{\|} \bar{P}\left(X_{\|}, \tau\right)\right\rangle$ & & Figure 7.15 \\
& $\left\langle v_{\|} \bar{P}\left(X_{\perp}, \tau\right)\right\rangle$, & \\
\hline Second moment in $v$ & $\left\langle v_{\|}^{2} \bar{P}\left(X_{\|}, \tau\right)\right\rangle$ & & \\
& $\left\langle v_{\|}^{2} \bar{P}\left(X_{\perp}, \tau\right)\right\rangle$, & & \\
\hline
\end{tabular}

Table 7.1: A table showing the independent, non-zero, displacement-resolved tensors available for the nonlocal dispersion measurements.

ventional numbering as in 7.16. In general a row describes components with the same displacement encoding direction and a column describes a similar velocity or correlation encoding. The exceptions are panel (e), which contain terms describing a longitudinal-transverse correlation and panel (f) which describes the transverse correlation orthogonal to a transverse displacement encoding. The complete components are also summarised in table 7.1 which is organised in a similar fashion. With the exception of figure 7.14 the extra tensors do not contain any information correlating velocities in space and time and the superposition detailed in section 5.3.4 is not required to measure them.

Inspection shows that, for flow in a beadpack we have 21 independent, non-zero, displacement resolved quantities, including 6 nonlocal elements. In addition, two propagators, two VACF measurements and the moments $\left\langle u_{\|}^{2}\right\rangle,\left\langle u_{\perp}^{2}\right\rangle,\left\langle v_{\|}\right\rangle$and $\left\langle v_{\|}^{2}\right\rangle$ are available. All of this information can be obtained from 6 experiments. 

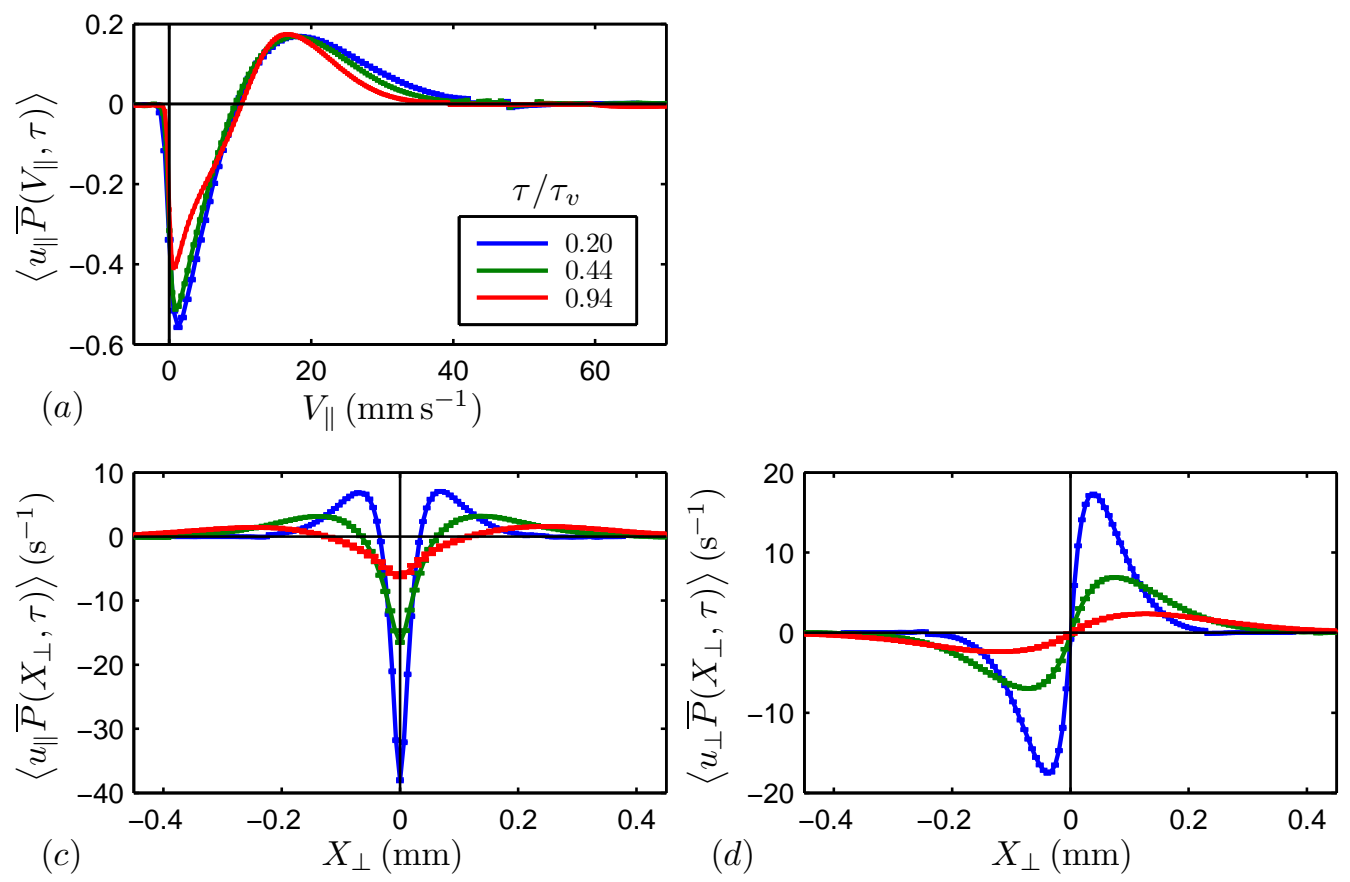

Figure 7.12: The displacement resolved first moments

\subsection{Conclusions}

We have demonstrated the ability to measure the six independent onedimensional nonlocal dispersion components for dispersive flow in a model porous medium. The components show remarkable structure that can be used to infer characteristics about the flow. Through our NMR measurements we have also been able to extract a range of other tensors. The addition of a further displacement encoding to provide a two-dimensional component was illuminating, an idea that will be expanded on in chapter 8 .

Simulations of the nonlocal dispersion tensor show broad agreement with experiment and will provide a useful tool for further investigations. Simulations naturally enable a more efficient means of investigating, over 

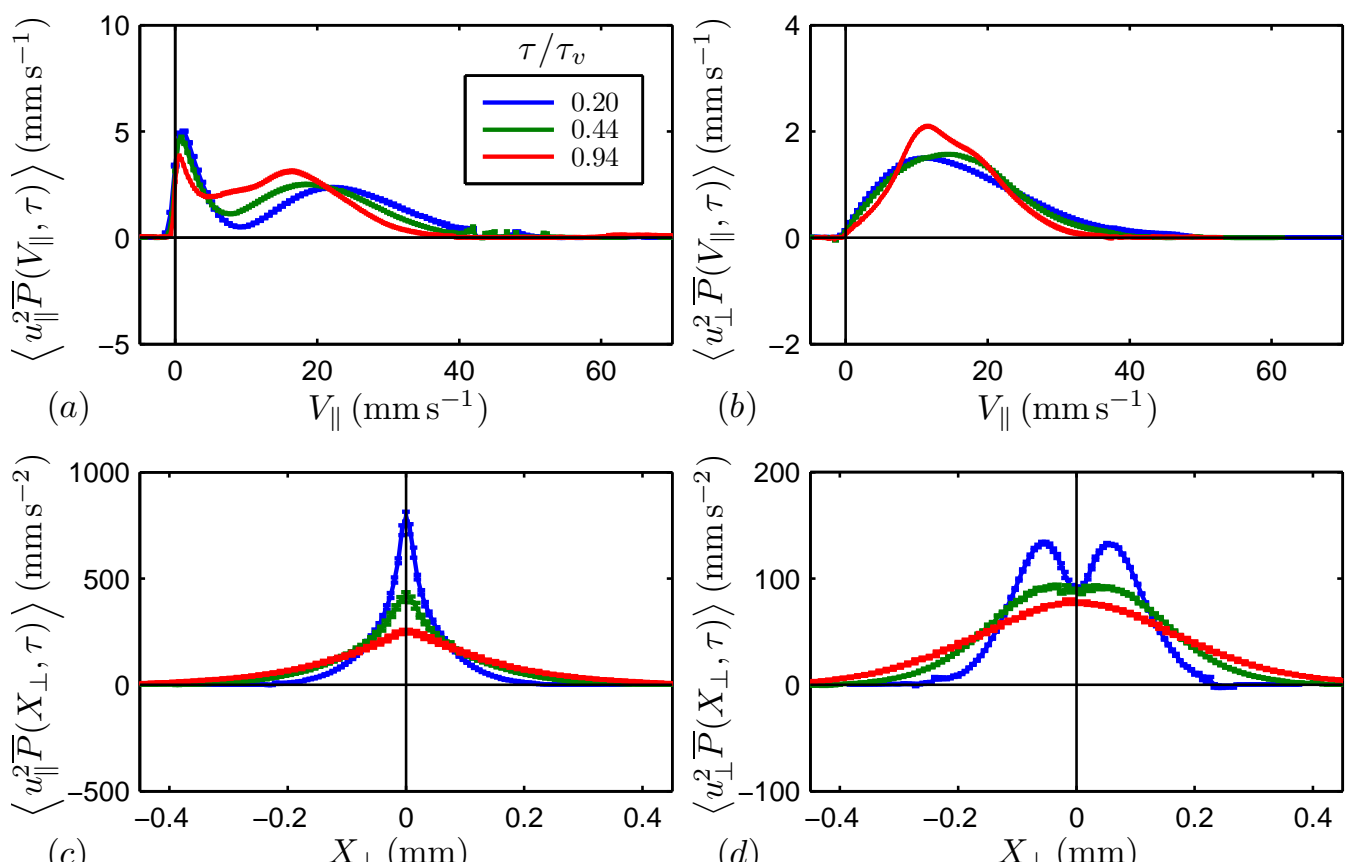

$(d)$

$X_{\perp}(\mathrm{mm})$

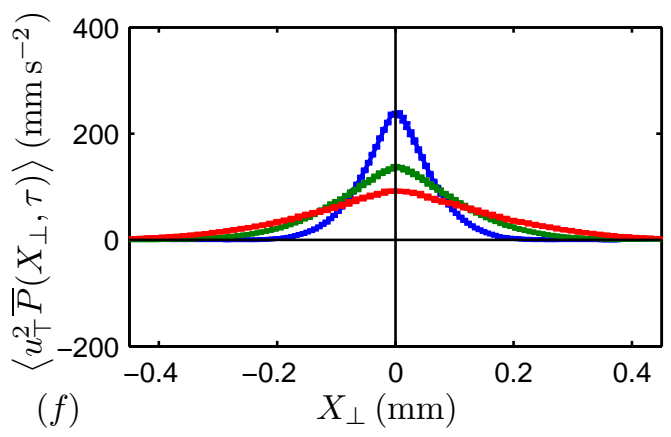

Figure 7.13: The displacement resolved second moments

a range of encoding times, the large-dimensional space involving multiple Cartesian directions. The direct comparison between the simulation and experiment can be improved in three ways: by matching the Reynolds number of the flows, by more accurately resolving the fine structure of the porous media, and by producing more precise estimates of the various observables. However, such simulations are computationally expensive 

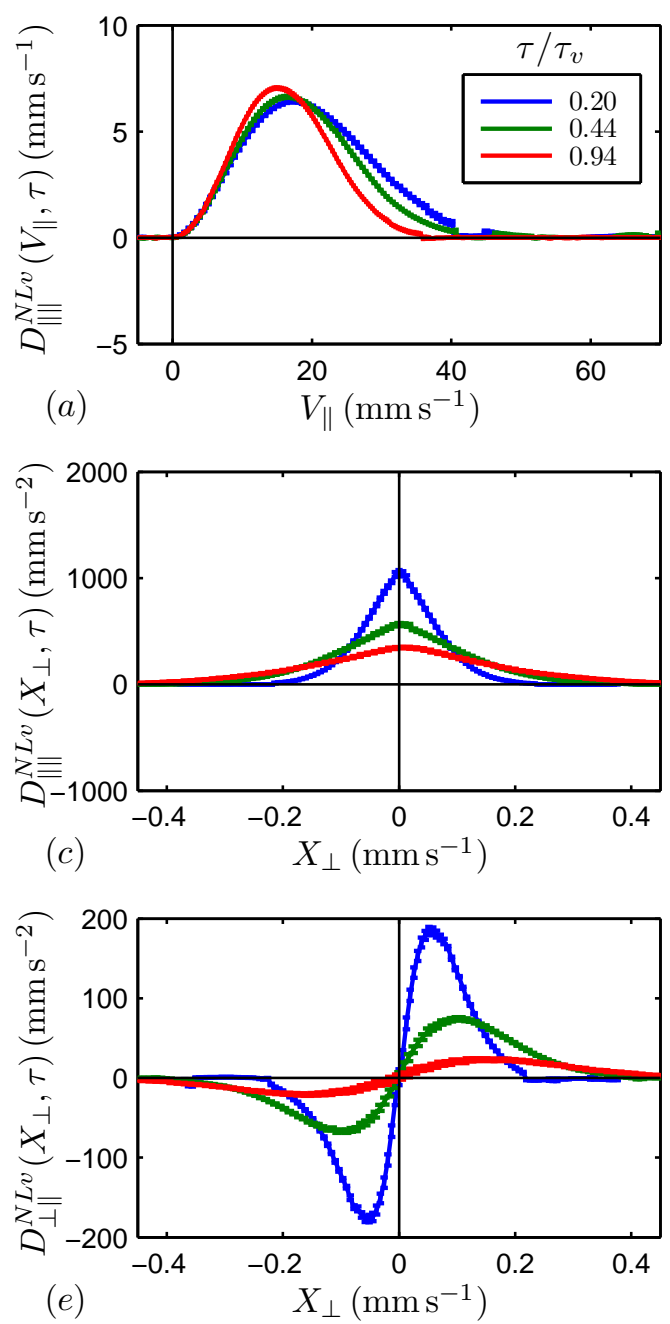

Figure 7.14: The nonlocal dispersion tensor in $v$

due to the requirement of very large lattices.

A remarkable feature of both experiment and simulation is the highly detailed structure of $\mathbf{D}^{N L}$, as well as the significant differences between the various tensor components. A simple picture involving flow around a single bead gives first order insight. But the structural details available suggest that by measuring $\mathbf{D}^{N L}$ we gain a highly sensitive probe of the 

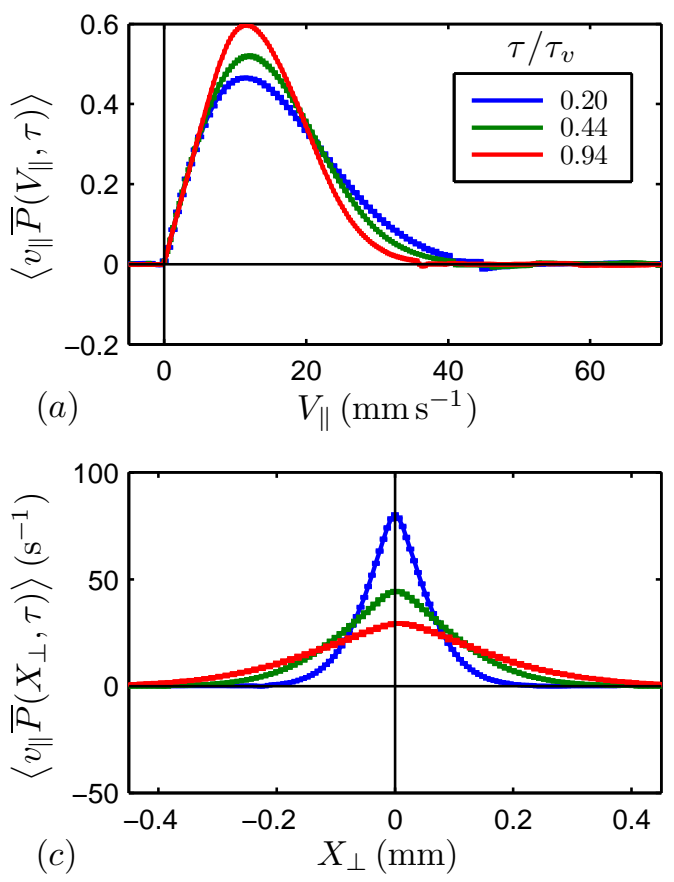

Figure 7.15: The displacement resolved second moments in $v$

mechanism of dispersion. Preliminary experiment of porous rocks show significantly different behaviour and are shown in chapter 9 . We anticipate that the $\mathbf{D}^{N L}$ measurements will provide a wealth of information. 

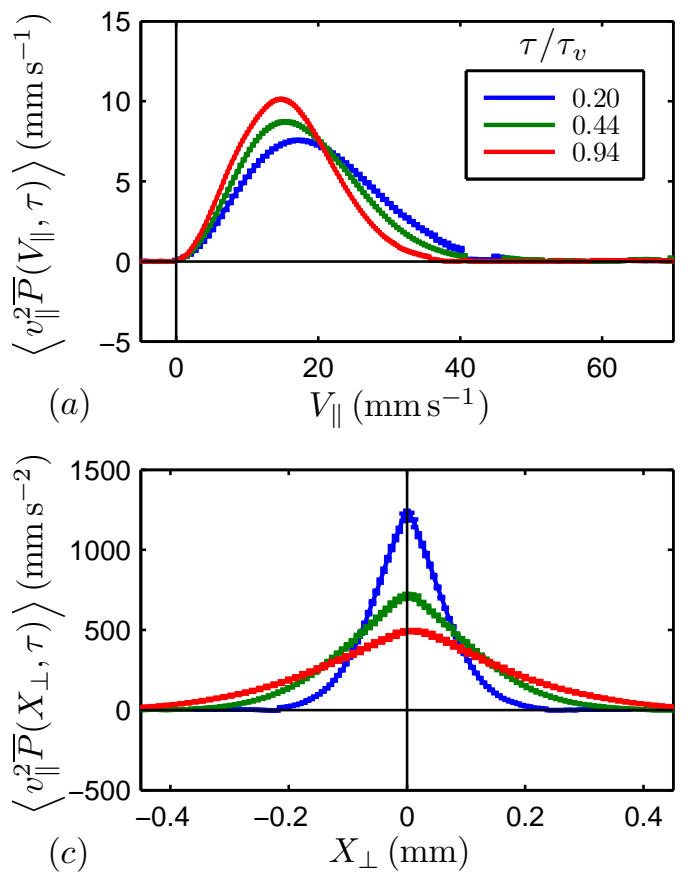

Figure 7.16: The displacement resolved first moments in $v$ 


\section{Chapter 8}

\section{Higher Dimensional Encodings}

\subsection{Introduction}

The one-dimensional nonlocal dispersion terms described in chapter 7 allowed us to infer some average properties of the flow. It was also seen that in NMR experiments one can resolve the displacement in two dimensions. In chapter 2, when the nonlocal dispersion tensor was first introduced, it was noted that in order to make predictions of the flux according to equation 2.28 the nonlocal dispersion tensor needs to be resolved in all three dimensions. As in equation 2.37 the one-dimensional terms measured in chapters 6, 7 and 9 are projections of the complete three dimensional term. A three dimensional nonlocal dispersion term will be denoted by $D_{\alpha \beta}(\mathbf{R}, \tau)$ where $\alpha$ and $\beta$ denote the chosen directions of velocity correlation.

The necessary encoding for an experimental measurement of such a term is prohibitively time consuming. Measurement of a single term at one encoding time could take 10 s of days. The computational time required for enough tracers to get meaningful statistics is also significant but not prohibitive. A large number of simulations can be run in parallel and each simulation can give a number of components at a broad range of encoding times. 
The simulations were undertaken as a means for investigating the three dimensional nonlocal dispersion tensor as a way of further describing some characteristics of the flow. The investigations contained here are in keeping with terms that could, in principle, be measured with NMR.

\subsection{Dimensionality}

For flow in porous media the displacement and velocity encoding can either be transverse to the flow or longitudinal. We have seen that the transverse directions do need to be treated independently because $D_{\perp \perp}^{N L}\left(X_{\perp}, \tau\right) \neq$ $D_{\perp \perp}^{N L}\left(X_{\top}, \tau\right)$ where $\perp$ and $\top$ indicate the two directions orthogonal to each other and the main flow. The component $D_{\perp \|}^{N L}\left(X_{\perp}, \tau\right)$ (See figure 7.4(f)) clearly shows structure that is otherwise hidden since the correlation function, $\left\langle u_{\perp}(0) u_{\|}(\tau)\right\rangle$ is equal to zero. This same correlation is described in the $D^{N L}$ component $D_{\perp \|}^{N L}\left(X_{\|}, \tau\right)$ but this is observed to be zero. Since some structure is present in the former quantity, perhaps a two-dimensional displacement encoding could provide more information, indeed the twodimensional component $\left\langle u_{\perp}(0) \bar{P}\left(X_{\perp}, X_{\|}, \tau\right) u_{\|}(\tau)\right\rangle$ shows this structure. Further investigation reveals eight independent two-dimensional components, namely $D_{\perp \perp}^{N L}\left(X_{\top}, X_{\perp}, \tau\right), D_{\|\|}^{N L}\left(X_{\top}, X_{\perp}, \tau\right), D_{\top \perp}^{N L}\left(X_{\top}, X_{\perp}, \tau\right)$ and $D_{\| \perp}^{N L}\left(X_{\top}, X_{\perp}, \tau\right)$ for fully transverse displacements and $D_{\perp \perp}^{N L}\left(X_{\perp}, X_{\|}, \tau\right), D_{\top \top}^{N L}\left(X_{\perp}, X_{\|}, \tau\right)$, $D_{\|\|}^{N L}\left(X_{\perp}, X_{\|}, \tau\right)$ and $D_{\| \perp}^{N L}\left(X_{\perp}, X_{\|}, \tau\right)$ which include a longitudinal displacement encoding.

The quantity $D_{\top \perp}^{N L}\left(X_{\top}, X_{\perp}, \tau\right)$ is interesting since none of the $D^{N L}$ showed any structure for orthogonal transverse correlations. The symmetry of $D_{\top \perp}^{N L}\left(X_{\top}, X_{\perp}, \tau\right)$ shows that the integrals over each dimension will indeed give zero. It is somewhat difficult to interpret correlations where orthogonal velocity encodings are used since it is unknown whether the positive correlation results from particles moving from a region where the velocity is in one direction to a region where the velocity is in the second direction or the particle has continued to moved in a way where the velocity is non- 


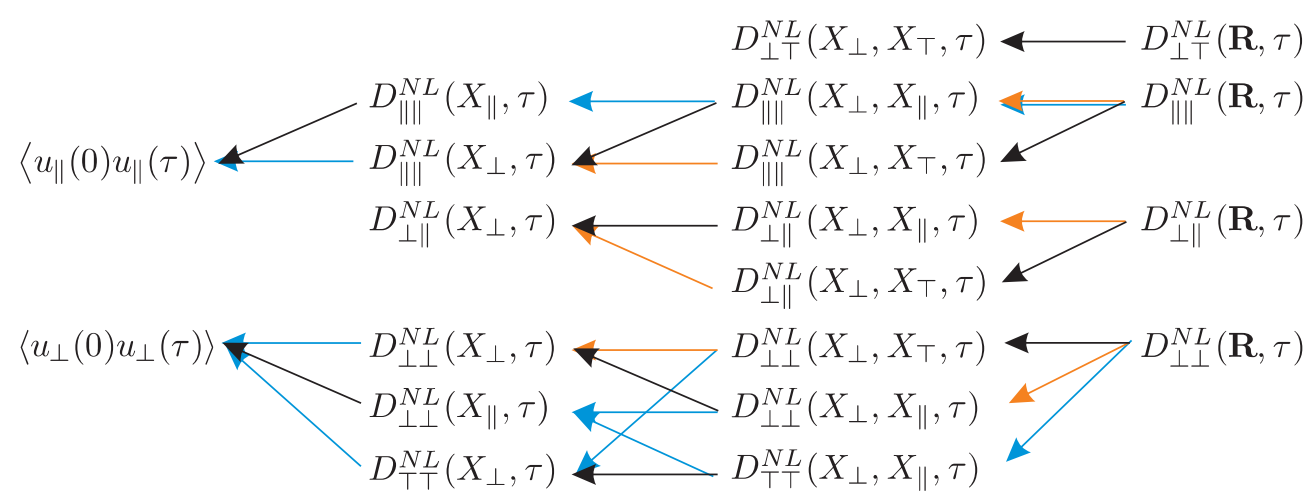

Figure 8.1: The relationship between the one-, two- and three-dimensional nonlocal components. The cyan arrow represent integration over $X_{\perp}$, the orange arrow represents integration over $X_{\top}$ and the black arrow represents integration over $X_{\|}$.

zero in both directions. Nevertheless, these quantities are available to be measured.

The six one-dimensional components describing the nonlocal dispersion in porous media have now become eight two-dimensional components. This extra information is still incomplete since there will still be terms that describe the same correlation but projected through different axes, such as $D_{\perp \perp}^{N L}\left(X_{\top}, X_{\perp}, \tau\right)$ and $D_{\perp \perp}^{N L}\left(X_{\perp}, X_{\|}, \tau\right)$. A full three-dimensional displacement resolution would result in only four terms, each describing a different correlation. These terms are $D_{\perp \perp}^{N L}(\mathbf{R}, \tau), D_{\perp \top}^{N L}(\mathbf{R}, \tau), D_{\perp \|}^{N L}(\mathbf{R}, \tau)$ and $D_{\|\| \|}^{N L}(\mathbf{R}, \tau)$. For flow in porous media, these terms represent the full nonlocal dispersion tensor.

The hierarchy of the one-, two-, and three-dimensional terms is shown in figure 8.1. The nonlocal term $D_{\perp T}^{N L}(\mathbf{R}, \tau)$ has no one-dimensional 'grandchild' and any terms describing the correlation $\left\langle u_{\perp}(0) u_{\|}(\tau)\right\rangle$ give a VACF equal to zero. Therefore only two of the three-dimensional components are direct expansions of non-zero velocity autocorrelation functions. 


\subsection{Simulation}

The simulation suite detailed in chapter 4 was used to statistically generate the three-dimensional components. In this chapter a finer resolution of 20 lattice sites per bead was used for the lattice Boltzmann simulation. An algorithm was used to plug small gaps to ensure hydrodynamic stability of the lattice Boltzmann simulation. A beadpack with a height of approximately 20 bead diameters was contained in a cylinder with a radius of 10 bead diameters. Time units will be given in units of time to flow one bead diameter, $\tau_{v}$, and displacement units will be non-dimensionalised by the bead diameter.

The method for generating three-dimensional nonlocal components is a further generalisation of that described in section 4.2.4. The contribution to a given correlation is calculated for each tracer, and then added to a bin dependent on the vector displacement. To generate meaningful statistics for a nonlocal component resolved in three dimensions a large number of tracer particles need to be simulated. A total of $4.2 \times 10^{7}$ were used to generate the plots in this chapter. Calculations were made for encoding times of $0.1,0.2,0.5,1,2,5$ and $10 \tau_{v}$, although the largest encoding time shown here will be $5 \tau_{v}$. This represents approximately 650 hours of processing time spread between 10 processors.

\subsection{Visualisation}

The components tend to show correlations and anticorrelations spread relatively broadly over regions of displacement space. Therefore the most effective way of visualising these terms is using three-dimensional isosurfaces. The components seem to describe regions of correlation that increase in intensity to a maxima. This results in each iso-surface representing a stronger correlation than that which it is inside. The isosurfaces are coloured red for a correlation and blue for an anticorrelation. The isosur- 
faces have been chosen to represent equally spaced values of correlation, straddling zero, covering the range of the correlation. Isosurfaces describing a low value of correlation will typically occupy a large region and may be susceptibly to simulation noise. Regions of intense correlation will be characterised by multiple isosurfaces packed together. The surfaces will all be plotted with the same transparency, so that intense correlations will quickly become opaque.

The value of each iso-surface will not be reported but the shape, and subsequent time evolution, will give qualitative information about the nature of the correlation function and its dependance on displacement.

\subsection{The Components}

\subsubsection{Longitudinal Correlations}

In section 7.5.4 the one-dimensional component $D_{\|\|}^{N L}\left(V_{\|}, \tau\right)$ was further investigated by monitoring the growth of the three distinct correlation regions. These regions can also be seen in the term $D_{\|\| \|}^{N L}(\mathbf{R}, \tau)$. With better statistics it is assumed that this term would be fully rotationally symmetric around the $V_{\|}$axis. The three-dimensional term does not show significant structure in the two transverse directions. This means that integrating the regions in the one-dimensional term approximates the size of regions in three dimensional displacement space reasonably well. At $\tau=1$ there is a slight overlapping in $V_{\|}$of the first correlation and the anti correlation.

As in the one-dimensional case, the $X_{\|}$axis is most conveniently displayed as velocity, here we can see the anticorrelation appearing at around $\tau=0.2$ and growing, displaying the same maximum intensity as that shown in figure 7.6. The three-dimensional component effectively shows the broadening of the correlation region that is moving faster than average. At short times, the fast moving particles have a strong correlation and have moved little in the transverse directions. At around $\tau=1$ this 

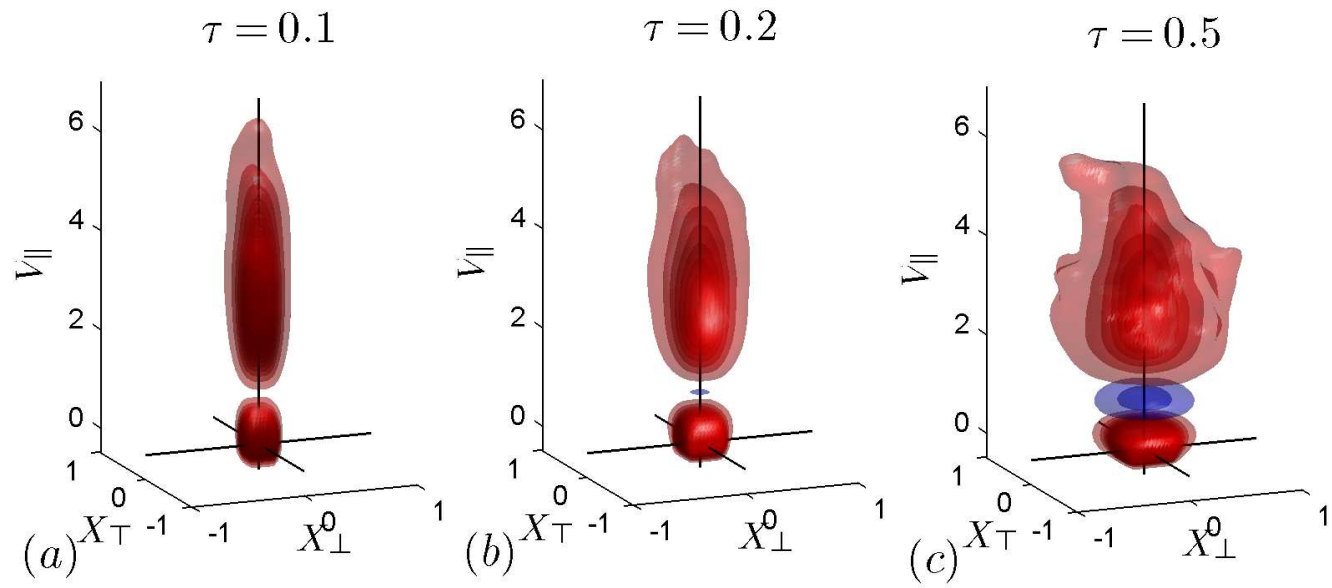

$$
\tau=1
$$

$$
\tau=2
$$

$$
\tau=5
$$
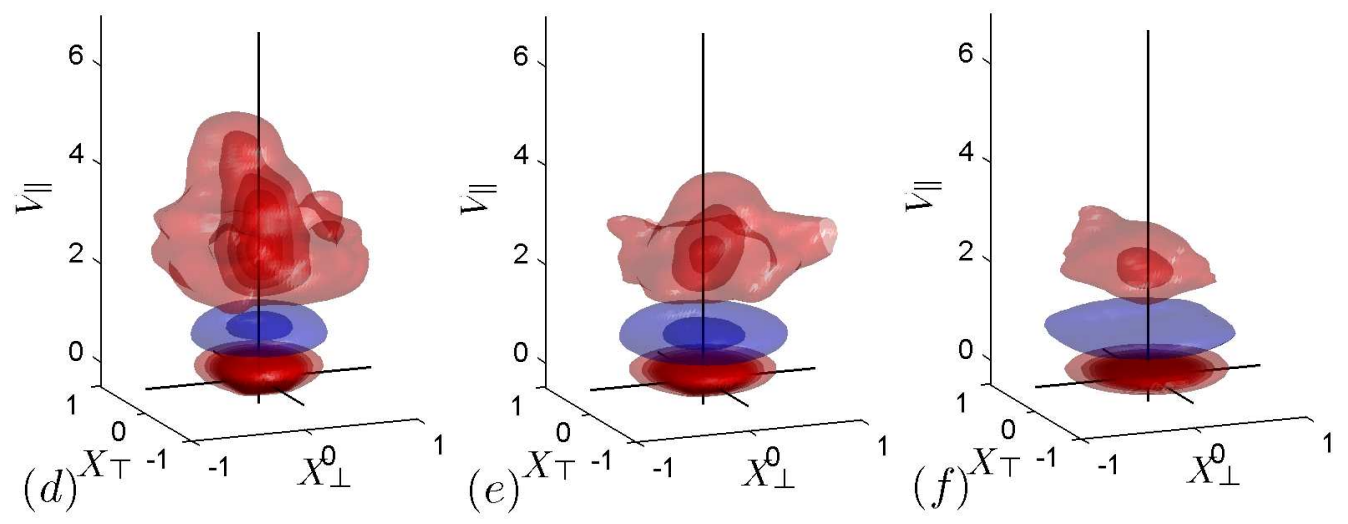

Figure 8.2: The time evolution of the component $D_{\|\| \|}^{N L}(\mathbf{R}, \tau)$. The longitudinal axis is plotted as velocity. The asymmetries in the low-value isosurfaces are due to simulations noise. The red regions correspond to a positive correlation and the blue region corresponds to an anticorrelation. 
region becomes quite spread out, but still measurable. Note that the isosurfaces in figures 8.2(c-e) are showing a significant amount of simulation noise. This is also the encoding time where the anticorrelated region appears to occupy the broadest region. According to the analysis given in figure 7.8 the maximum contribution from the anticorrelation region is around $\tau=2.5$. At encoding times of greater than 2, the second correlation region becomes significantly less broad in transverse displacement space and velocity space. Throughout the encoding times the correlation region around 0 , becomes slightly broader in the transverse directions and still accounts for a large contribution to the velocity autocorrelation function. In all of the plots in figure 8.2 the visualisation of the intensity of this correlation is saturated due to the large number of partially transparent iso-surfaces.

\subsubsection{Transverse Correlations}

In contrast to the component describing fully longitudinal correlations, all the other three-dimensional components are best displayed on a displacement axis in the longitudinal direction. This suggests that any effects from components that have a transverse encoding are closely related to displacement phenomena, while the effects from component $D_{\|\| \|}^{N L}$ are related to velocity phenomena.

The time evolution of the component $D_{\perp \perp}^{N L}(\mathbf{R}, \tau)$ is shown in figure 8.4. An experimentally measured two-dimensional component $D_{\perp \perp}^{N L}\left(X_{\perp}, X_{\|}, \tau\right)$ can be seen in figure 7.11. The three dimensional component evolves to a shape that is not unlike a protea flower (depicting the anti correlation) with two leaves (depicting the correlation). To aid in visualisation three views of the iso-surfaces at $\tau=0.5$ are shown in figure 8.3. The symmetry of this component is such that

$$
\begin{aligned}
D_{\perp \perp}^{N L}\left(X_{\perp}, X_{\top}, X_{\|}, \tau\right) & =D_{\perp \perp}^{N L}\left(X_{\perp},-X_{\top}, X_{\|}, \tau\right) \\
& =D_{\perp \perp}^{N L}\left(-X_{\perp}, X_{\top}, X_{\|}, \tau\right)
\end{aligned}
$$



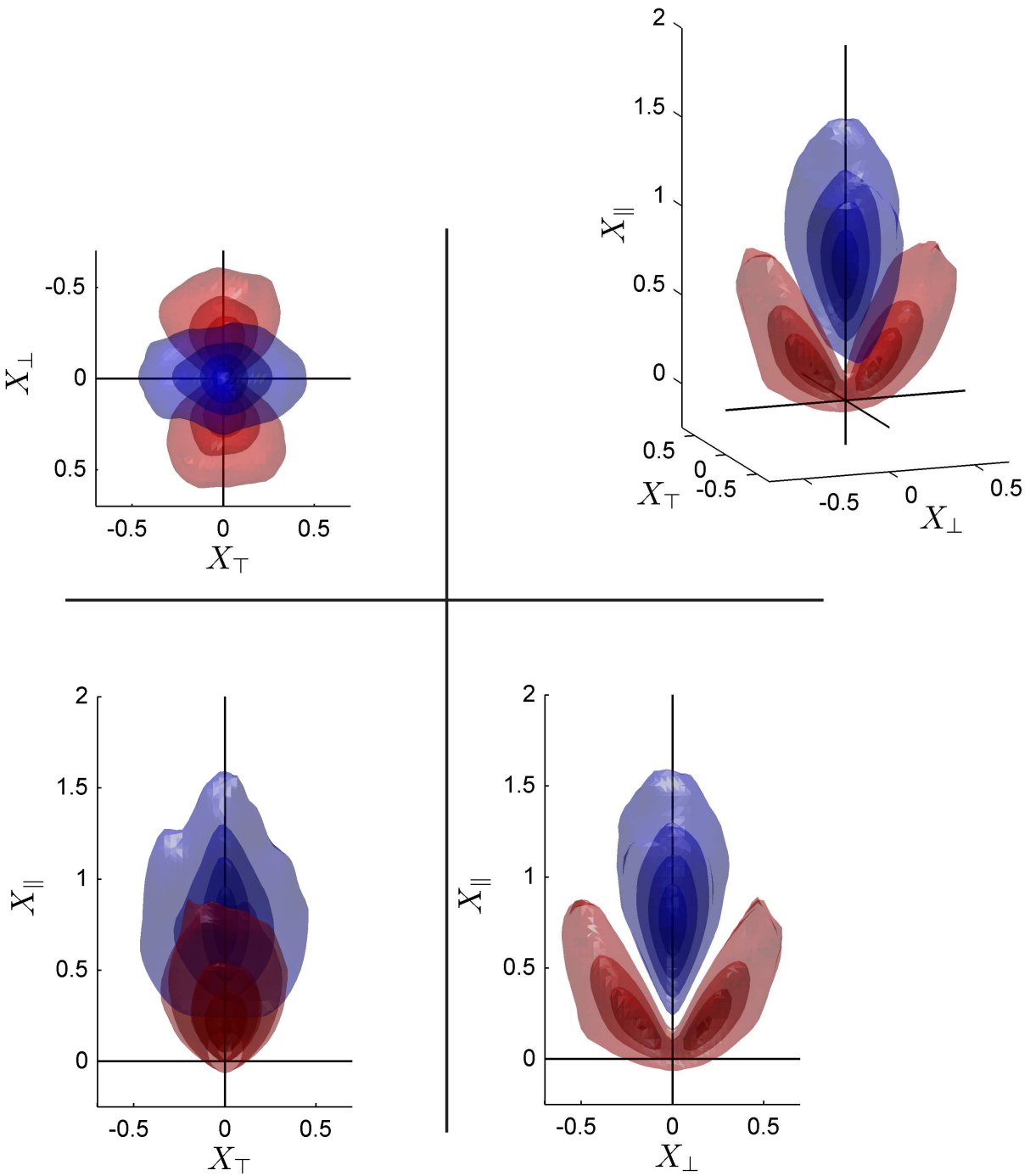

Figure 8.3: The component $D_{\perp \perp}^{N L}(\mathbf{R}, \tau)$ for $\tau=0.5$ visualised in three planes as well as the three dimensional view. The structure of this term is not unlike a protea flower. 


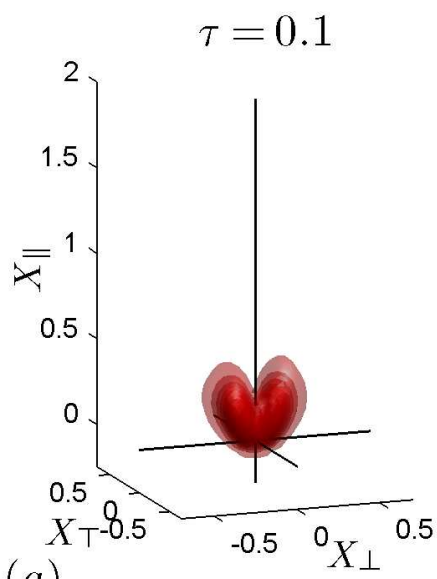

(a)

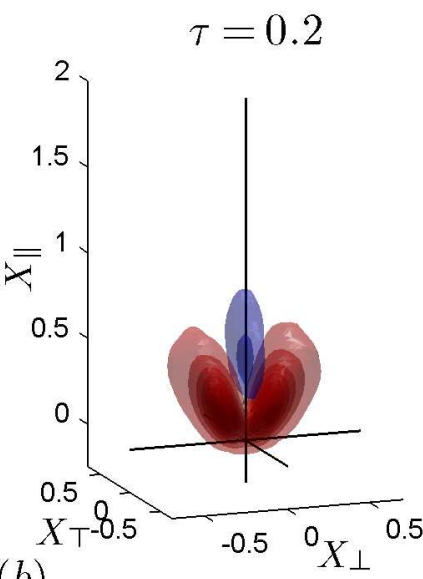

(b)

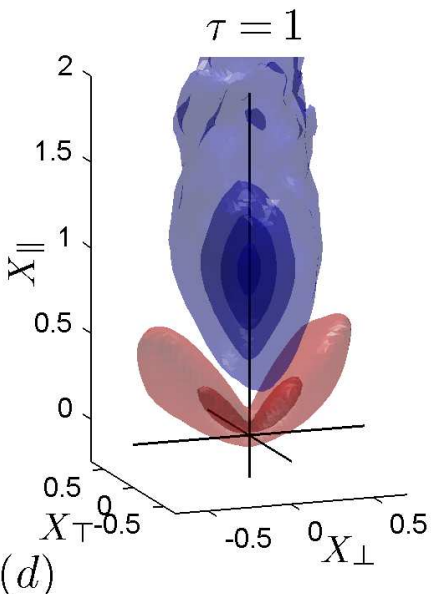

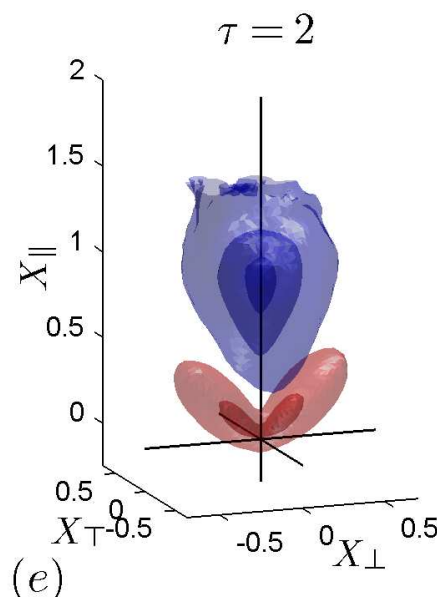

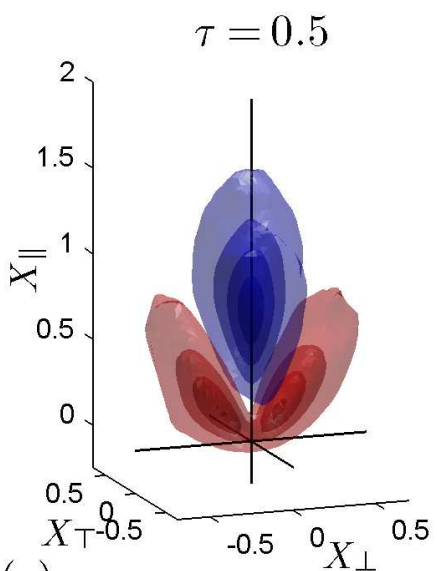

(c)

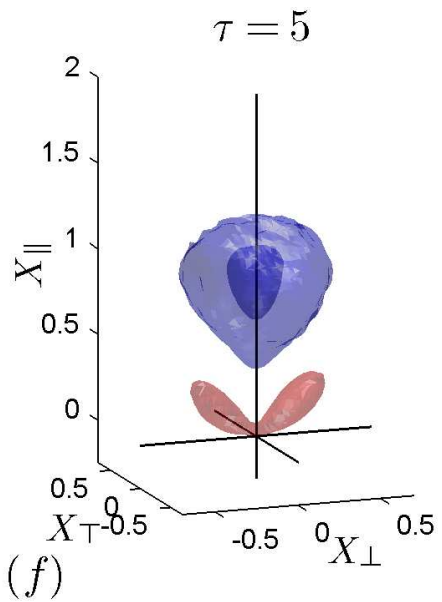

Figure 8.4: The time evolution of the component $D_{\perp \perp}^{N L}(\mathbf{R}, \tau)$. The shape of the correlation regions appear to be a function of displacement rather than velocity. 
to within simulation noise.

Initially the transverse velocities, $u_{\perp}$ are highly correlated, particularly in the $X_{\perp}$ displacement direction. The 'heart shape' in figure 8.4a shows that this is true for a range of displacements in the $X_{\|}$direction greater than the average. At encoding times greater than 0.2 , an anticorrelation region appears, this region starts to spread out, particularly in the $X_{\top}$ direction. The center of this region remains at a displacement of one bead diameter, and then dies away, becoming largely symmetric around the $X_{\|}$ axis in the process. The 'petal' shape of the correlation region, populated by particles that have moved between zero and half a bead diameter simultaneously in $X_{\perp}$ and $X_{\|}$, appears to cover a maximum range of displacements at 0.5 and then die away. There is no significant broadening in the $X_{\top}$ direction for this region.

It is worth noting that these figures show that for long encoding times, such that particles have moved on average five bead diameters, there is still a subset of particles that show transverse correlations. These particles can be further divided into two sets that have moved some small distance around a bead and those that have, on average, swapped their $u_{\perp}$ component of velocity while being displaced by a bead diameter. We can conclude that particles that have moved more than two bead diameters have, on average, lost all memory of their initial velocity. One might expect a 'recorrelation' of $u_{\perp}$ for particles that have moved two bead diameters but no evidence of that is seen here. This may be a function of some structural ordering of the beadpack. The transverse correlation effects that remain for $\tau>2$ are the result of a small set of particles that have been displaced by an amount comparable to a bead diameter.

The regions of correlation and anticorrelation can be separately integrated and monitored as a function of time. This is shown in figure 8.5. The decay of the positively correlated particles is nearly mono-exponential with a time constance of approximately $3.5 \tau_{v}$. The anticorrelated region has its maximum contribution at approximately $0.5 \tau_{v}$. This may seem to 


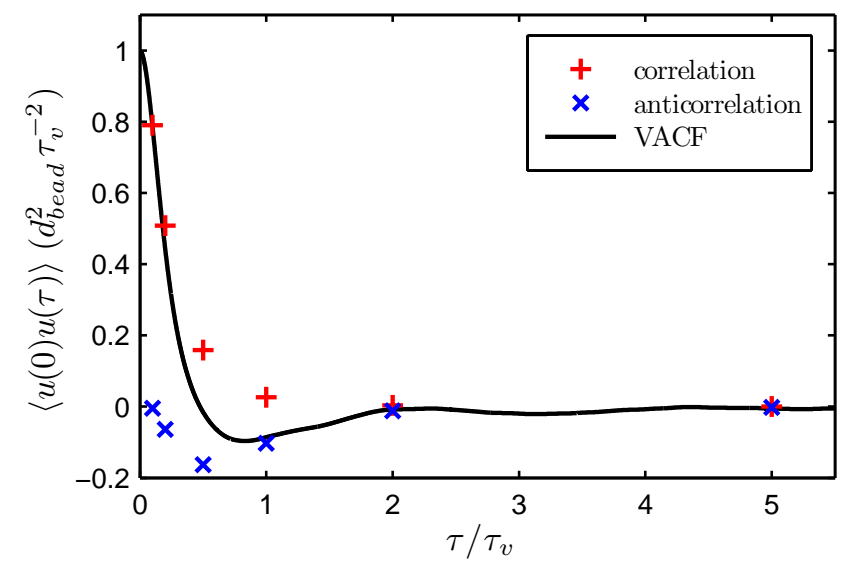

Figure 8.5: The time evolution of the correlation and anticorrelation regions of $D_{\perp \perp}^{N L}(\mathbf{R}, \tau)$ as shown in figure 8.4. The decay of the positive correlation is nearly monoexponential and the negative correlation has a maximum contribution at approximately $0.5 \tau_{v}$.

not be in agreement with what is shown in figure 8.4(d), however it is important to note that the visualisation in figure 8.4(d) shows that the anticorrelation region covers the largest displacement space, but the contours between panels are not equivalent. The intensity of the respective correlations must be found from integration. We can see therefore, that the maximum anticorrelation of transverse velocities occurs at $0.5 \tau_{v}$ and is made up of particles that have displaced only a small amount in the transverse direction. The longitudinal displacement of these particles cover a range that is centered around one bead diameter, that is, greater than the average displacement.

\subsubsection{Transverse-Longitudinal Correlations}

The component $D_{\perp \|}^{N L}(\mathbf{R}, \tau)$ has no correlation function associated with it, but it does have a single one-dimensional grandchild. It is this term $D_{\perp \|}^{N L}$ that showed largest differences in the comparison between simulation and 
experiment in chapter 7 . The symmetry of this component is such that

$$
\begin{aligned}
D_{\perp \|}^{N L}\left(X_{\perp}, X_{\top}, X_{\|}, \tau\right) & =-D_{\perp \|}^{N L}\left(-X_{\perp}, X_{\top}, X_{\|}, \tau\right) \\
& =D_{\perp \|}^{N L}\left(X_{\perp},-X_{\top}, X_{\|}, \tau\right)
\end{aligned}
$$

to within simulation noise.

It is somewhat difficult to interpret correlations that compare orthogonal velocities since it is impossible to tell if a strong correlation is due to a particle moving from a region with a velocity in $u_{\perp}$ say, to a region with a velocity $u_{\|}$, or due to a particle that has moved on a diagonal with the same starting and finishing velocity. Nevertheless, the presence of a correlation is indicative of some discernable structure.

The term $D_{\perp \|}^{N L}(\mathbf{R}, \tau)$ follows a similar evolution to that of $D_{\perp \perp}^{N L}(\mathbf{R}, \tau)$. There is little spreading in the direction of $X_{\top}$ and the overall pattern appears to grow, initially, with the bulk flow and then stabilise at $\tau=1$ and die away.

At short times there are four distinct regions, these show that particles displaced further than the average have, on average, a $u_{\|}$velocity correlated with the $u_{\perp}$, in the direction of their displacement $X_{\perp}$. The particles displaced less than the average also show a $u_{\|}$velocity correlated with the $u_{\perp}$, in the direction of their displacement $X_{\perp}$, but the correlation is shown as an anticorrelation by virtue of the fact that the $u_{\|}$velocities will be negative. At an encoding time of $\tau=0.5$ there is still a measurable correlation between transverse and longitudinal velocities for particles that have moved further than the average.

The inspection of this three-dimensional term helps to understand the origin of the correlation peaks shown in figures 7.4(e) and 7.3(e). The large correlation and peaks around $X_{\perp}=0$ in the measurement are not present in the simulation. Based on the assumption that the measured $D_{\perp \|}^{N L}(\mathbf{R}, \tau)$ would have similar structure to that shown in figure 8.6, the correlation peaks are due to particles that have moved a short distance in $X_{\|}$. This 


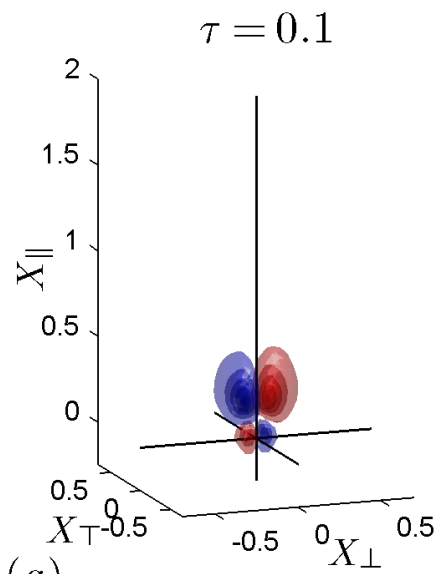

(a)

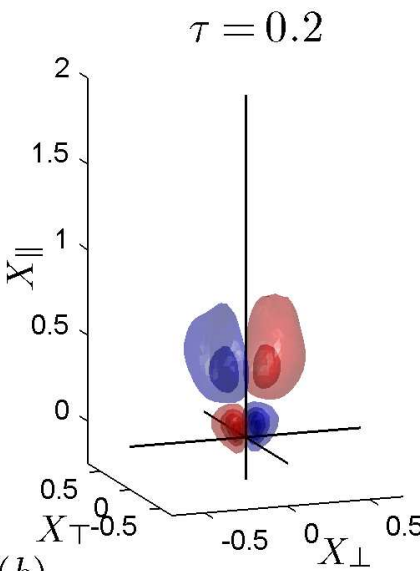

(b)

$$
\tau=1
$$

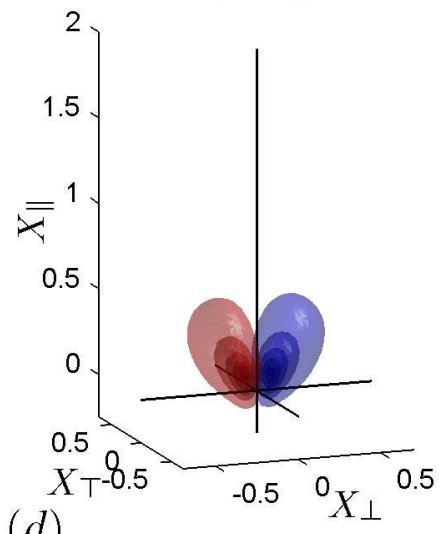

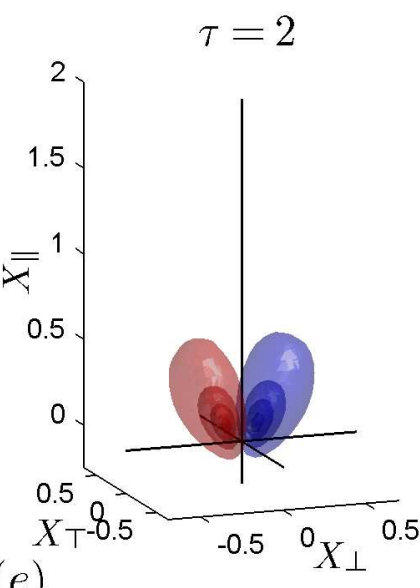

(e)

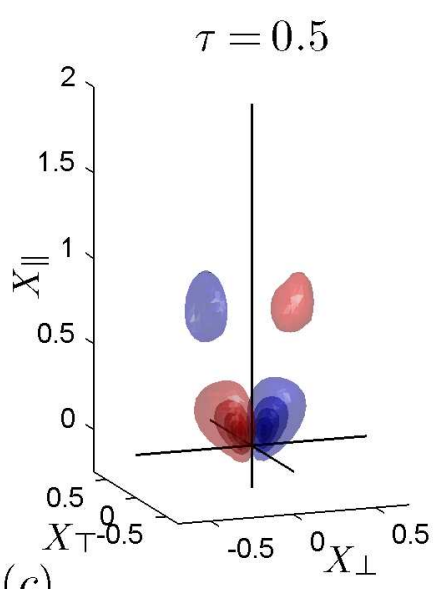

(c)

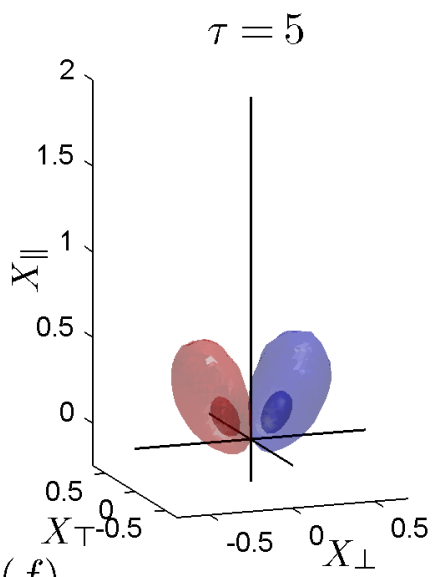

Figure 8.6: The time evolution of the component $D_{\perp \|}^{N L}(\mathbf{R}, \tau)$. The $X_{\perp}-X_{\|}$plane of symmetry and no correlation corresponds to no net velocity in the $X_{\perp}$ direction. The surface of zero correlation in the $X_{\perp}-X_{\top}$ plane at a non-zero $X_{\|}$corresponds to the bulk flow. 


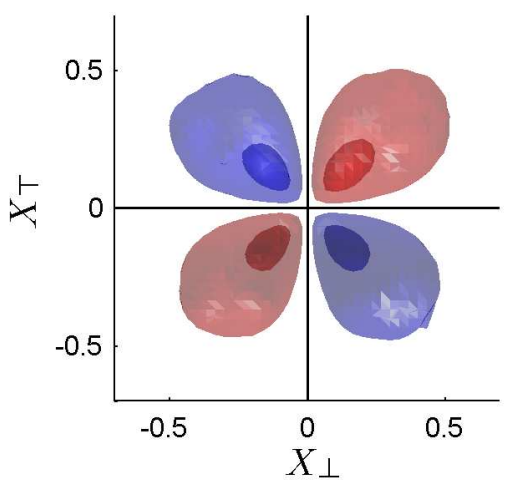

Figure 8.7: Visualising the component $D_{\perp \top}^{N L}(\mathbf{R}, \tau)$ in the $X_{\perp}-X_{\top}$ plane.

means that, on average, the short time, small displacement diagonal flow behaviour around a bead is significantly different between the simulated and measured beadpacks.

\subsubsection{Transverse-Transverse Correlations}

The component $D_{\perp \top}^{N L}(\mathbf{R}, \tau)$ is an unusual term, it has no one-dimensional 'grandchildren' and the pronounced features, similar to $D_{\perp \|}^{N L}(\mathbf{R}, \tau)$ but in the $X_{\perp}-X_{\top}$ plane, are a result of the distinction between the orthogonal directions. A view of the $X_{\perp}-X_{\top}$ plane is shown in figure 8.7. The symmetry is such that

$$
\begin{aligned}
D_{\perp \top}^{N L}\left(X_{\perp}, X_{\top}, X_{\|}, \tau\right) & =-D_{\perp \top}^{N L}\left(X_{\perp},-X_{\top}, X_{\|}, \tau\right) \\
& =-D_{\perp \top}^{N L}\left(-X_{\perp}, X_{\top}, X_{\|}, \tau\right) .
\end{aligned}
$$

The shape appears to stabilise at around $0.5 \tau_{v}$ and the range of the correlation regions is restricted, in all dimensions, to approximately half a bead diameter. The structure of the three dimensional term suggests that a one-dimensional component would be measurable if the displacement 

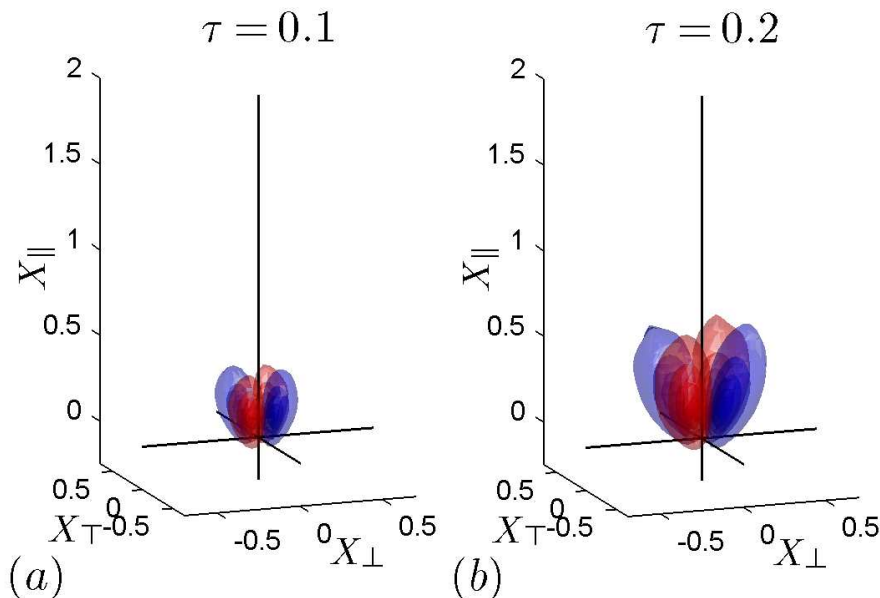

(b)
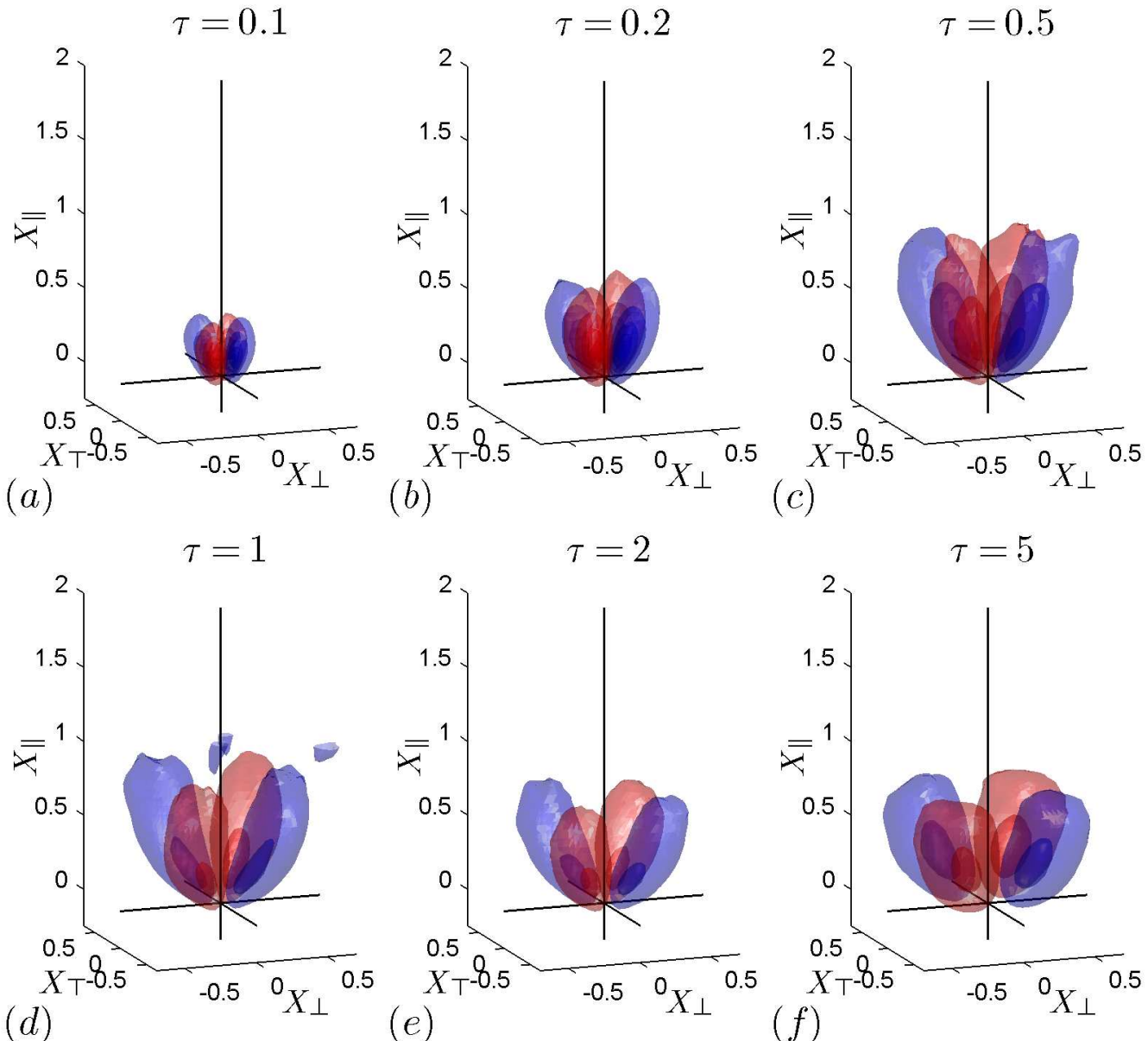

Figure 8.8: The time evolution of the component $D_{\perp \top}^{N L}(\mathbf{R}, \tau)$. The symmetry is such that there are no one-dimensional components associated with this correlation. 


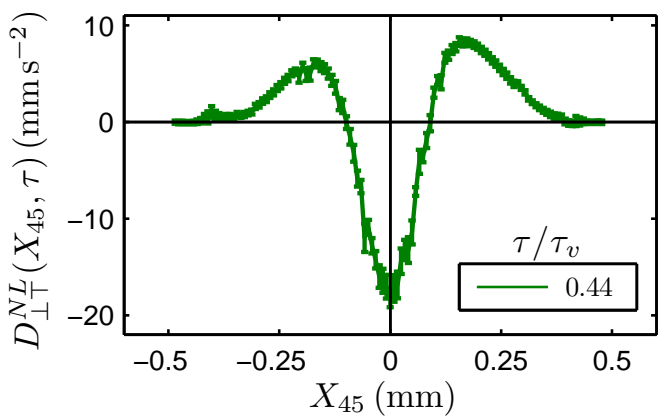

Figure 8.9: An orthogonal transverse correlation can be observed in the displacement resolution aligned at $45^{\circ}$ to both velocity encoding directions. This experimental result is for the same configuration is detailed in chapter 7 .

resolution was along the axis $X_{\perp}=X_{\top}$, that is, at a $45^{\circ}$ angle to both velocity encoding directions. Such an experimental measurement has been done, shown in figure 8.9 under the same experimental conditions as described in chapter 7 . This confirms the structure seen in simulation and may help to quantify the behaviour of transverse correlations.

\subsection{Conclusions}

The three-dimensional nonlocal dispersion components allow the visualisation of displacement resolved correlations without the averaging that is inherent in the one- and two-dimensional projections.

It is clear that the fully transverse correlations are restricted to particles that have only moved a short distance. Once a particle has moved more than $1.5 d_{\text {bead }}$ all the correlation effects have disappeared. The correlation shape that remains, at short displacements, appears to show relatively constant structure over an encoding time of $0.5-5 \tau_{v}$. This could help to describe a 'signature' of the flow and diffusion pattern around a sphere. It would be illuminating to investigate the differences in the same term for 
flow in a pack of prolate or oblate spheres. The displacement structure of the correlations is perhaps a function of the grain shape. The components containing orthogonal correlations are also, for the most part, restricted to short displacements.

The fully longitudinal components once again enabled us to look at three different correlation regions. The particles that moved faster than the mean flow begin to occupy a broad displacement space at around $\tau=0.5-1$. Following this, the particles that contribute to the anticorrelation begin to occupy a larger region in the transverse displacement space. These features are a measure of the tortuosity of the flow paths.

The symmetry and structure of the three-dimensional terms suggests that should a high degree of information be required and a full measurement unobtainable, a compromise could be made. There is lack of detailed structure in the $X_{\top}$ direction for most of the terms and hence the set of components $D_{\|\|}^{N L}\left(X_{\|}, \tau\right), D_{\perp \perp}^{N L}\left(X_{\|}, X_{\perp}, \tau\right)$ and maybe $D_{\| \perp}^{N L}\left(X_{\|}, X_{\perp}, \tau\right)$, would contain most of the information that would be contained in a full set of three-dimensional measurements. 


\section{Chapter 9}

\section{Nonlocal Dispersion in a Rock Core}

\subsection{Introduction}

NMR techniques for characterising porous media have found widespread application in measurements of rock cores. The study and further understanding of the pore structure and flow characteristics in rock cores provides essential information with regards to the economics of oil recovery. Commercial logging of potential oil-wells is commonplace [163; 164], making use of a one-sided access permanent magnet based probe [165; 166]. NMR techniques used to characterise rock cores include; one- and two-dimensional relaxation measurements [32; 34; 167; 168], static gradient techniques [32;169], pulsed gradient techniques NMR [170] including imaging [171], dispersion measurements [172] and time-of-flight methods using hyper-polarised Xenon gas [119]. The presence of internal gradients [173] requires the development of new techniques to either eliminate unwanted effects [89; 174] or to take advantage of them [32].

The type of rock studied here, a Bentheimer sandstone, has been widely studied in the laboratory using NMR. Examples include; the measurement flow propagators [123; 175-178], determining volume-to-surface ra- 
tios [40] and the monitoring the approach to asymptotic dispersion conditions [29]. Benthiemer is considered a 'heterogeneous' porous medium [29] but it is also 'considered to have a fairly uniform and reproducible pore structure' [179].

Here we demonstrate the ability to measure a number of one-dimensional nonlocal dispersion tensor components for low Pe, low Re number in a Bentheimer rock core. Measurements are performed at several encoding times which are well in the preasymptotic regime.

\subsection{Experimental Setup}

\subsubsection{Flow Cell}

A flow cell containing a bentheimer rock core was supplied by Dr Mike Johns of Cambridge university. A rock core of diameter $12.3 \mathrm{~mm}$ and length $40 \mathrm{~mm}$ was encased in perspex. A thickness of 'Scotch Brite' was used at either end to provide some uniform flow distribution. The length of the rock core was long enough to ensure that it extended outside the sensitive volume of the $20 \mathrm{~mm}$ diameter birdcage RF coil.

The core was saturated under vacuum for 24 hours with a solution of $3 \mathrm{wt} \% \mathrm{KCl}$. As in the experiments on the beadpacks (section 7.2), the water was doped with $0.025 \mathrm{wt} \% \mathrm{GdCl}_{3}$ to reduce the $T_{1}$ to approximately $110 \mathrm{~ms}$. The porosity was determined by weighing the sample before and after saturation. The measured value of $27 \pm 1 \%$ is slightly higher than some published data [29; 179], but not incompatible with others [40]. The grain size, and hence a measure of characteristic length, of Bentheimer is reported as around $300 \mu \mathrm{m}$ [29]. The average pore size, as measured by NMR, has been reported as $25 \mu \mathrm{m}$ [175] or bimodal with $79 \%$ of the pore space described by a volume-to-surface ratio ranging from $6-30 \mu \mathrm{m}$ and $21 \%$ from pores with a volume-to-surface ratio of less than $4 \mu \mathrm{m}$.

A small amount of unwanted signal originates from somewhere in the 
cell. The signal comes from some ${ }^{1} \mathrm{H}$ nuclei that are completely stationary resulting in an artifact at $X=0$ for all propagator and nonlocal measurements.

\subsubsection{Flow Stability}

The high permeability of the rock core rendered the pump system used in chapter 7 unsuitable even for a modest flow rate of $1.0 \mathrm{~mm} \mathrm{~s}^{-1}$. Instead, a Pharmacia P-500 dual syringe pump was used. This class of pump can produce long time scale stability at high pressure but produces very short time scale variations and has a flow 'dead-time' of 1-2 s while the pistons are reversed approximately every $300 \mathrm{~s}$. In order to provide a stable flow rate during this time a flow damper with a time constant of the order of 100 s of seconds is needed. A length of rubber tubing was found to be suitable. The pressure build up in the rubber tube was enough to provide a nearly constant flow rate during the reversal and still provide long time stability. There were however, still some remaining flow instabilities, resulting in modest error bars and a small amount of scatter in the nonlocal dispersion measurements.

The stability of flow is sufficient for reliable propagator measurements. Shown in figures 9.2(a, c, e, g) are the two indistinguishable propagators extracted from the uncompensated and the compensated experiments. The repeatability of the propagator measurement in comparison to the nonlocal components highlights the need for a very stable flow rate.

\subsubsection{Internal Gradients}

The difference in susceptibility between the solid matrix and the permeated fluid results in a distribution of internal gradients. These internal gradients, which are sometimes as large as the pulsed gradient, present some challenges to implementation of NMR experiments. To minimise unwanted diffusive attenuation a 'Cotts' [89] sequence (also called BP- 
PGSTE) is typically used for displacement encoding. An alternative approach to minimise the internal gradients is to work at a lower field. It has been shown that $2 \mathrm{MHz}$ is a suitable field to minimise internal gradients while still providing good signal to noise [178]. The majority of PGSE laboratory measurements of rock cores are at around $85 \mathrm{MHz}$ [29; $123 ; 175 ; 177]$. The experiments described in this chapter were performed at $400 \mathrm{MHz}$ hence the internal gradients are expected to be large. In the displacement encoding dimension of the pulse sequence a BP-PGSTE is implemented, however, the timings are not ideally suited for minimising internal gradients. It is expected that these internal gradient effects will make quantitative measurements difficult.

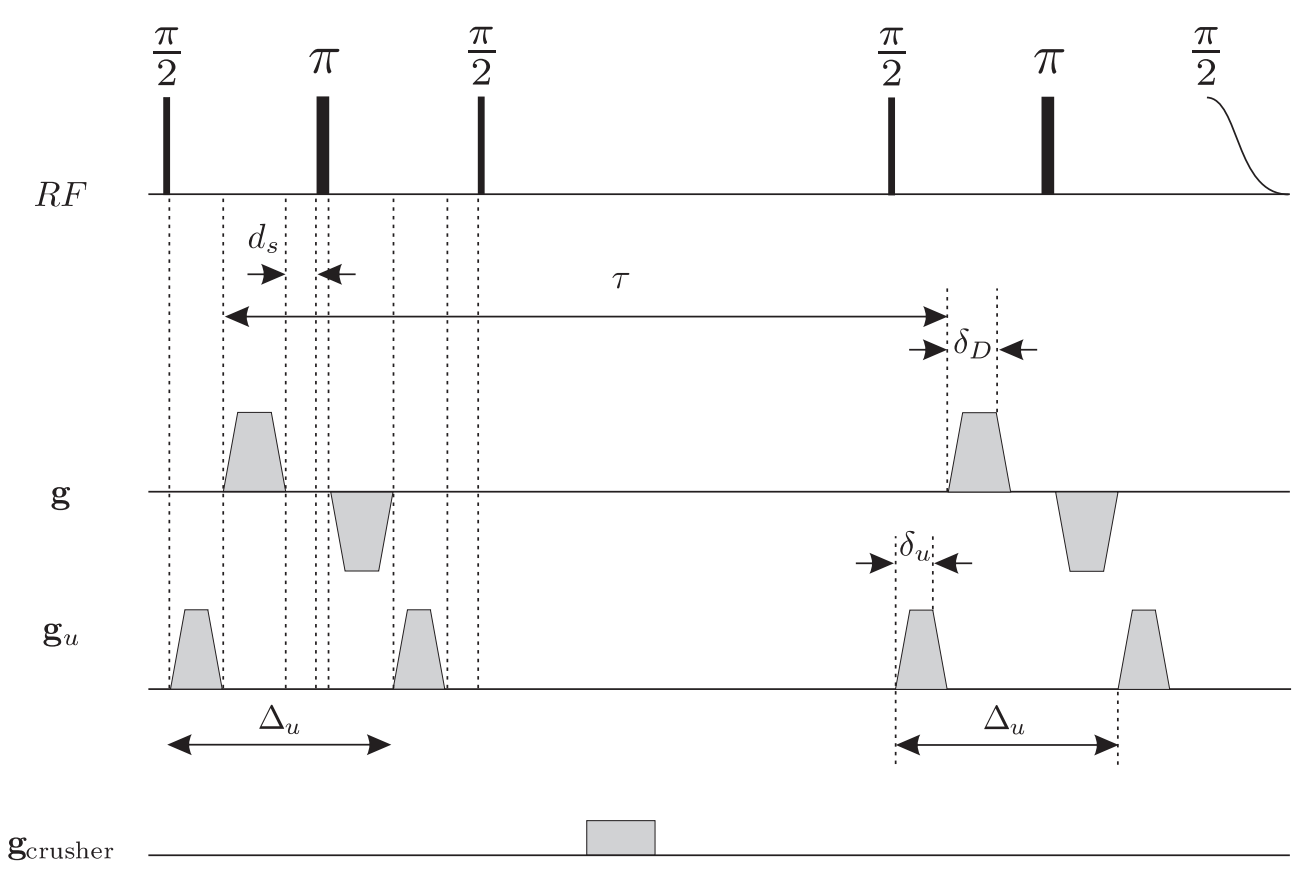

Figure 9.1: The modified pulse sequence to allow for use of the maximum gradient the system can provide. The gradient pulses for the motion encoding and the displacement encoding are no longer conincident. 


\section{Pulse Sequence Modification}

The pulse sequence described in chapter 5 was used for measurements in chapters 6 and 7. Several modifications needed to be made in order to perform successful measurements on a rock core. The short length scales and slower velocities require larger values of $\mathbf{q}$ to achieve full attenuation in the displacement dimension and sufficient attenuation in the motion encoding dimension.

In the motion encoding dimension we have $\mathbf{q}_{u}=\gamma \delta_{u} \Delta_{u} \mathbf{g}_{u}$, the maximum $\mathbf{g}_{u}$ being determined by the experimental apparatus, in this case $1.45 \mathrm{~T} \mathrm{~m}^{-1}$. To achieve appropriate attenuation we then need to increase either $\Delta_{u}$ or $\delta_{u}$ or both. In order to take advantage of the maximum available gradient, the motion encoding and the displacement encoding are now separated in time. To achieve a maximum $\Delta_{u}$ while minimising the time in the transverse plane the motion encoding pulses are either side of the bi-polar displacement encoding pulses. In an ideal 'Cotts' sequence the gradient pulse in the encoding dimension would be equidistant in time between the RF pulses. If this timing was used here the total time in the transverse plane would be too long, instead all time in the transverse plane has been minimised. This is shown schematically in figure 9.1. For the experiments shown here we have used $\delta_{u}=0.65 \mathrm{~ms}, \delta_{D}=0.65 \mathrm{~ms}$ with a stabilisation delay of $0.5 \mathrm{~ms}$. This results in $\Delta_{u}=3.55 \mathrm{~ms}$.

It is important to note the disparity in the compensation on internal gradients for each dimension. A BP-PGSTE is used for the displacement encoding but each velocity encoding is implemented as a standard PGSE. For consistency the same pulse sequence and pulse times were used for all measurements even if the motion encoding and displacement encoding were parallel, or mixing times such that a lower gradient allowed for coincident pulses.

The measurement of the displacement encoding used a graining of the gradients similar to that described in section 5.4.1 and reference [114]. For measurements where the displacement encoding is longitudinal to the 
flow a graining scheme consisting of 8 fine, 18 medium and 26 coarse steps was used giving a total of 53 gradient values. The step ratios were 1:2:8 giving an interpolated data set with 253 values. For measurements where the displacement encoding is transverse to the flow a graining scheme consisting of 8 fine, 16 medium and 16 coarse steps was used giving a total of 41 gradient values. The step ratios were 1:2:8 giving an interpolated data set with 169 values. In all experiments 25 steps were used in the velocity encoding dimension.

\subsubsection{Measured Components}

The figures showing the one-dimensional nonlocal dispersion components for flow in a bead pack suggest that the majority of the information and structure is contained in the terms $D_{\|\|}^{N L}\left(V_{\|}, \tau\right), D_{\perp \perp}^{N L}\left(V_{\|}, \tau\right)$ and $D_{\perp \perp}^{N L}\left(X_{\perp}, \tau\right)$. These are the components that have been measured here. Measurement of the full set of components and higher dimensional terms required for a more complete analysis of the fluid behaviour is reserved for further work.

\subsection{Nonlocal Components}

\subsubsection{Longitudinal Correlations}

Shown in figure 9.2 are the longitudinal propagators (a, c, e, g) and the nonlocal components $D_{\|\| \|}^{N L}\left(V_{\|}, \tau\right)(\mathrm{b}, \mathrm{d}, \mathrm{f}, \mathrm{h})$ for encoding times of 15.0, 32.4, 69.8 and $150 \mathrm{~ms}$. These times are all relatively short in comparison to the time to flow a characteristic length, in this case $300 \mathrm{~ms}$. At this low Peclet number of 53 the mixing is dominated by diffusion and we are clearly in the preasymptotic regime. Figure 9.2(a) shows the velocity propagator at $15 \mathrm{~ms}$ which shows an amount of diffusive broadening. The propagator measured at the longest time (figure 9.2(g)) agrees well with the propagator measured at a comparable encoding time in reference [29]. 
At this encoding time there appears to be a local minima in the correlation described by $D_{\|\| \|}^{N L}\left(V_{\|}, \tau\right)$ around the mean flow rate. In contrast with the beadpack measurements and simulations, this minima does not develop into an anticorrelation. We presume that this is due to the displacement at this Peclet number and encoding times being dominated by diffusion effects. That is, on average, the particles that moved with the average have done so under diffusive effects, their initial and final velocities are not correlated enough to require a slower final velocity in the case of a fast initial velocity.

One striking feature of the components shown in figure 9.2 is the distinction between particles that have not moved far and those that have moved further than the average. The time evolution of the shape of $D_{\|\| \|}^{N L}\left(V_{\|}, \tau\right)$, closely matches that of the propagator, further describing a subset of particles that are static or very slow moving. From the $D_{\|\| \|}^{N L}\left(V_{\|}, \tau\right)$ measurements we can directly see the effect on the VACF and hence dispersion.

The particles than have moved faster than the average show contributions to the VACF that are spread over a wide range of displacements. The nonlocal dispersion components clearly shows there are a subset of particles that move at high velocities, around six times faster than the average. This subset of particles is difficult to identify in the propagators.

\subsubsection{Transverse Correlations}

Figure 9.3 shows two components that describe the transverse correlation; $D_{\perp \perp}^{N L}\left(X_{\|}, \tau\right)$ in panels $(\mathrm{a}, \mathrm{c}, \mathrm{e}, \mathrm{g})$ and $D_{\perp \perp}^{N L}\left(X_{\perp}, \tau\right)(\mathrm{b}, \mathrm{d}, \mathrm{f})$. As was seen in chapter 8 the components describing transverse correlations appear to show structure that is related to the displacement rather than velocity. This suggests that we may be able to infer some kind of length scale dependent characteristic correlations.

The regions of displacement showing a high degree of correlation in figures $(\mathrm{a}, \mathrm{c}, \mathrm{e}, \mathrm{g})$ all show a similar shape. For the four encoding times 

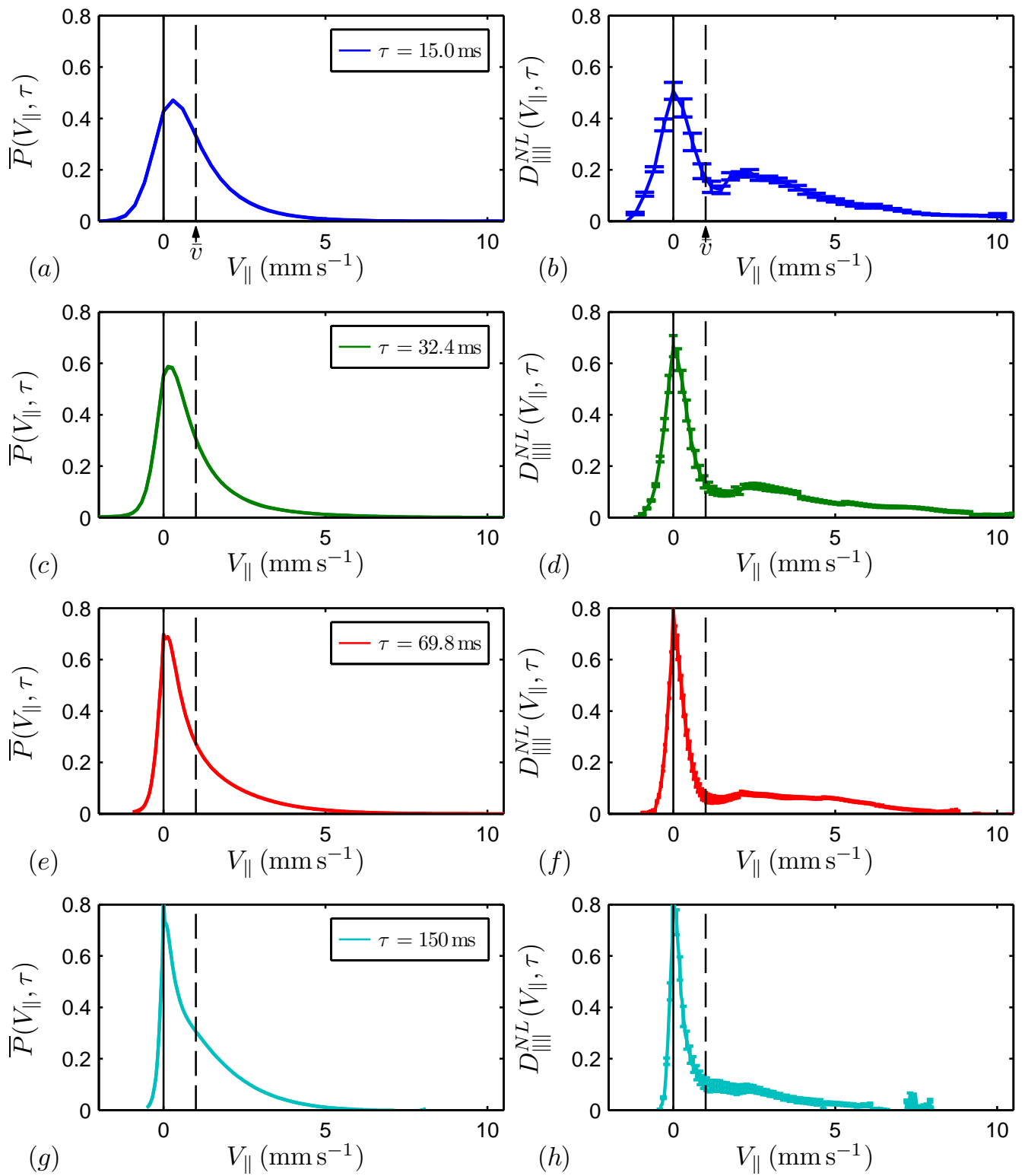

Figure 9.2: The measured propagators (a,c,e,g) and nonlocal dispersion (b,d,f,h) for longitudinal encoding for flow in a Bentheimer rock core. These components are plotted as a function of $V_{\|}$. The average velocity, $1.0 \mathrm{~mm} \mathrm{~s}^{-1}$, is shown as a dotted line. The Peclet number was 53. 
the average displacement of the particles will be $15 \mu \mathrm{m}, 32.4 \mu \mathrm{m}, 69.8 \mu \mathrm{m}$ and $150 \mu \mathrm{m}$. Despite this, the correlation region is quickly built up by particles that have been displaced by $0-200 \mu \mathrm{m}$. Apart from the decay in magnitude of this term there is little development of the shape. In contrast to the beadpack measurements it is difficult to determine any anticorrelation regions in the term $D_{\perp \perp}^{N L}\left(X_{\|}, \tau\right)$. The component $D_{\perp \perp}^{N L}\left(X_{\perp}, \tau\right)$ shown in figure $9.3(\mathrm{~d}, \mathrm{f}, \mathrm{h})$ describes the same correlation, here the anticorrelation regions are clearly identifiable for particles around $X_{\perp}=0$.

Assuming that the three-dimensional nonlocal component describing these transverse correlations has a similar from to that shown in figure 7.10, we can make some assumptions about the flow. Of course in the onedimensional measurements a certain amount of structure has been averaged out. The anticorrelated region shown around $X_{\perp}=0$ is most likely distributed over a range of longitudinal displacements and may be centered around a characteristic length. The correlations regions seem to occupy a similar displacement space relatively independent of encoding time. In the $X_{\perp}$ direction the correlations are limited to around $200 \mu \mathrm{m}$. The extent of the correlation regions in the $X_{\|}$direction can not be determined, but it can be seen that they are averaged out by the anticorrelated region.

\subsection{Conclusions}

The investigations of the nonlocal dispersion tensor components for flow in a beadpack lead to expectation that the most information can be seen in the three terms $D_{\|\| \|}^{N L}\left(X_{\|}, \tau\right), D_{\perp \perp}^{N L}\left(X_{\|}, \tau\right)$ and $D_{\perp \perp}^{N L}\left(X_{\perp}, \tau\right)$. These three terms, at a small range of $\tau$ have been measured for low Peclet number flow in a single Bentheimer rock core.

The short distance scales in the rock core required changes to be made to the pulse sequence detailed in chapter 5 , the unwanted effects due to internal gradients have not been fully minimised. The lack of a suitable 

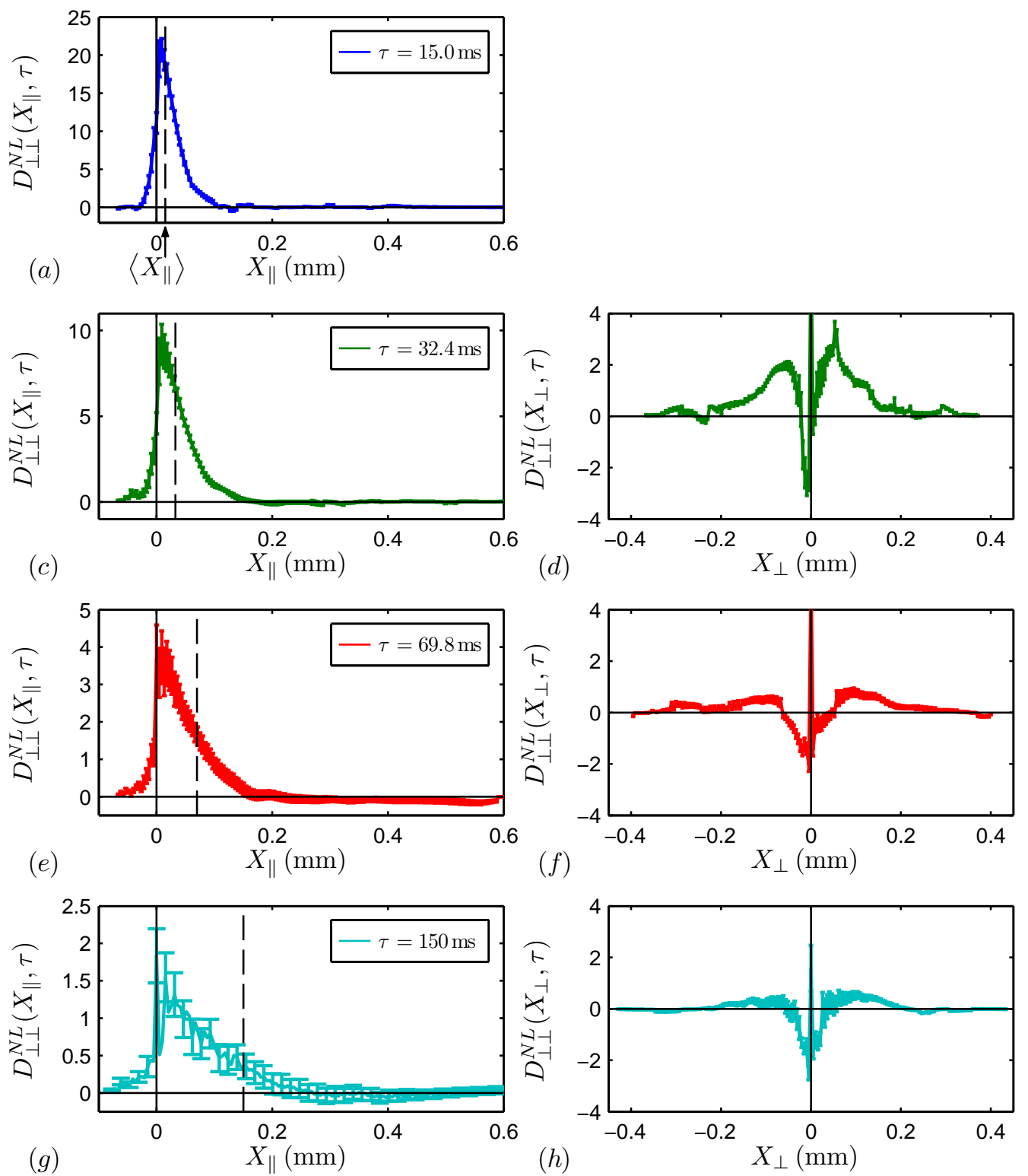

Figure 9.3: The components $D_{\perp \perp}^{N L}\left(X_{\|}, \tau\right)(\mathrm{a}, \mathrm{c}, \mathrm{e}, \mathrm{g})$ and $D_{\perp \perp}^{N L}\left(X_{\perp}, \tau\right)(\mathrm{d}, \mathrm{f}, \mathrm{h})$ describing transverse correlations for flow in a Bentheimer rock core.

mechanism for generating a stable flow resulted in large error bars and truncation errors in the $q^{2}$ fitting algorithm. These two drawbacks pre- 
cluded detailed, quantitative analysis of the various correlations and are obvious targets for further improvements. Nevertheless, the structure of the displacement resolved correlations can be inferred. The differences between the measurements on the Bentheimer rock core and the beadpack were clear.

At the low flow rate and short encoding times correlations can readily be described over displacements much smaller than the characteristic length and any REV. The components describing the transverse correlations were beginning to show a time-independent structure, in the displacement space, similar to that observed in the simulated three-dimensional components. A two-dimensional measurement here would be an obvious extension to further resolve the nature of the anticorrelated regions and investigate a characteristic displacement structure for correlations. 


\section{Chapter 10}

\section{Conclusions}

The importance of the nonlocal dispersion tensor in porous media should not be underestimated. For accurate predictions of concentration profiles and particle displacement for cases when Fick's law fails, a nonlocal theory, and hence a nonlocal dispersion tensor is crucial. We have chosen the nonlocal dispersion tensor of Koch and Brady [13] to measure using nuclear magnetic resonance. This quantity, even in cases where Fickian dispersion is observed, is well defined at the pore scale and provides a precursor to the velocity autocorrelation function and hence the dispersion tensor.

\subsection{The Work Contained in this Thesis}

\subsubsection{A New Measurement Technique}

A focus of this work was the development of a nuclear magnetic resonance pulse sequence that was capable of measuring the nonlocal dispersion tensor in porous media. The pulse sequence developed was a twodimensional sequence comprised of a double PGSTE experiment in one dimension and a BP-PGSTE in the second dimension. The use of the sequence and subsequent superposition and post-processing is entirely new 
and requires particular care. When developing a new technique one must be sure that the new measurement is 'correct'. Great care was taken to ensure that the NMR measurements were reliable and valid. Fortunately the PGSE NMR experiments implemented here provide data that can be checked for self-consistency. Temporal variations in the flow and spurious signals from the pulse sequence are easily identified, although not so easily removed.

The analysis in the double-PGSTE dimension provided the most challenges. A protocol needed to be developed to ensure a robust fit to $q$ and $q^{2}$ that was insensitive to noise and kept truncation errors to a minimum. The requirements on this process were further amplified due to the nature of the superposition. In order to extract the desired encoding the superposition is often a small difference between large numbers. Any attempt to measure nonlocal dispersion where; the flow was unstable, the data contained artifacts or an unstable $q^{2}$ fitting algorithm was used, would not be successful. The details of the technique have been published in Journal of Magnetic Resonance [180].

In order to verify the experimental technique several systems were investigated which provided calculable nonlocal dispersion components. The first, capillary flow in a pipe, proved to be a very difficult experiment. The second, flow in a Couette cell provided a more than satisfactory result. While not providing a dispersive system, the detailed structure was ideal for comparing experimental data with that calculated. These results, shown in figure 6.13, marked the first successful measurement, using any technique, of nonlocal dispersion tensor components and resulted in a publication in Physical Review Letters [181].

The use of the simulation suite as part of the methodology development was of great benefit. The echo attenuation simulation provided an ideal way to choose experimental parameters and more importantly design the algorithm for the low- $q$ fitting. The simulated experimental data could be compared directly with the statistically calculated quantities. The 
flexibility of the simulation suite also provided another tool for interpretation of the results.

\subsubsection{Analysis of Nonlocal Dispersion}

The new measurement technique was applied to flow in a porous medium, for the first time, resulting in six one-dimensional measurements. The summary of these components are given in figure 7.4, and the discussion of these results led to a publication in Physics of Fluids [158]. This set of figures demonstrates the vast information available in the nonlocal dispersion tensor, choosing one Peclet number and only three encoding times gives a set of six quantities all rich in features. There are two ways of describing longitudinal correlations, three ways of describing transverse correlations and, a way of describing transverse-longitudinal correlations.

The analysis of the terms containing transverse correlations led to a description of a characteristic flow behaviour at the pore scale. A twodimensional measurement helped to further inform on the behaviour and highlight the need for higher encodings.

The comparison between the measured nonlocal dispersion components and the simulated components showed similar structure but also gave an indication of the sensitivity of the nonlocal dispersion to pore scale behaviour. The large difference in the nonlocal term describing longitudinaltransverse correlations could be indicative of vastly different displacement behaviour at the pore scale.

The simulation suite provided a mechanism for investigating the parameter space that was either unavailable or inconvenient to measure experimentally. The time evolution of the three distinct regions in the fully longitudinal nonlocal term was investigated this way. These subsets of particles showed behaviour at different characteristic times.

The need for higher dimensional encodings was demonstrated, this was unfeasible experimentally but achievable in simulation. This lead 
to the identification of four three-dimensional nonlocal components for flow in porous media. These terms were investigated over a range of encoding times. It was shown that transverse correlation is provided solely by particles that have been displaced by a distance comparable to a pore. The transverse correlation terms appear to describe a characteristic flow around a bead. A small set of components was suggested to encompass most of the details of a full three dimensional set, namely the one-dimensional fully longitudinal component and a two-dimensional term describing transverse correlations.

A Bentheimer rock core was investigated by measuring some selected one-dimensional nonlocal components. Clear evidence of a transverse anticorrelation was seen for particles that had moved small distances in the transverse direction and a long way longitudinally. The fully longitudinal nonlocal term did not show the anticorrelation seen in the bead packs but clearly identified some fast moving particles and their contribution to the velocity autocorrelation function.

\subsection{Future Work}

\subsubsection{Experimental Developments}

In order for further experimental investigations to be undertaken several improvements to the pulse sequence could be made. The biggest advantages will come from improvements that reduce the total experimental time.

Presently, the need for completely separate experiments for different encoding times is cumbersome. Use of a technique such as 'difftrain' [122; 123], would enable convenient use for experimental time to obtain a range of encoding times. A decrease in signal-to-noise and a small reduction of flexibility in displacement encoding would be encountered. There may be further improvements to the displacement gradient graining technique, 
especially if two- and three-dimensional displacement encodings are used. The use of an efficient graining process in the motion encoding dimension, while keeping in mind the requirements for the low- $q$ fitting could be advantageous. Finally a more efficient phase cycle, perhaps based on the 'cogwheel' approach [182] could reduce the number of steps from the present 32. Should all these improvements be feasible it is not unreasonable that the experiment time could be reduced by a factor of 10 to 50 .

Further improvements to the experimental technique would include the use of the spectral domain, to simultaneously measure oil and water, and to include a relaxation dimension to compensate for any $T_{2}$ or $T_{1}$ distributions in complex porous media.

The further application of nonlocal measurements to rock cores needs a flow system with a greater stability. An improvement to the treatment of internal gradient could be made [174;183], including a thorough treatment in the motion encoding dimension. It may be most practical to move to a lower field. It is anticipated that measurements on rock cores will be the immediate follow up to this thesis.

\subsubsection{New Systems}

There is almost no limit to the range of dispersive systems to which this new measurement technique can be applied. There are several decades of Peclet number to be explored, each Peclet number requiring a full range of encoding times. The types of media to be investigated are numerous, heterogenous, bi- and poly-disperse beadpacks, hierarchical media and a whole range of rock cores. The differing pore and grain structure of each of these media are bound to show differing characteristic flow behaviour highlighted by nonlocal dispersion measurements.

A characteristic flow behaviour could be investigated conveniently in simulation. The apparent dependance on a pore or grain shape could be investigated with regular packings of beads, packings of prolate or oblate 
spheroids or bi-disperse beadpacks.

Measurements could be made with different probe fluids further increasing the parameter space. Hyper polarised gas would provide access to different length scales. Processes closer to that of industry could be investigated, examples include two phase flow such as gas/liquid or water and oil. Non-Newtonian fluids in porous media display different characteristic behaviour and would display different correlations also. The recent use of using oil-filled spheres for dispersion measurements of suspended colloidal particles $[157 ; 184]$ would provide an interesting system for nonlocal measurements.

\subsubsection{Nonlocal Theory of Koch and Brady}

Finally, the theory of Koch and Brady could be investigated by identifying an NMR measurable system displaying a clearly visible nonlocal effect and then, on measuring the full nonlocal dispersion tensor and using equation 2.28, predicting the measured effect.

\subsection{Closing Remarks}

While the word 'simulation' appears in the title of this thesis, the project itself was inherently experimental. The importance of the simulation suite to the project cannot be underestimated, and doubtless the project would have had very limited success without it. However the major accomplishments, and indeed representative of the time and effort, are the successful experimental design and measurements of, for the first time, The Nonlocal Dispersion Tensor. 


\section{References}

[1] A. E. Scheidegger, The Physics of Flow Through Porous Media. Uiversitu of Toronto Press, 1957.

[2] J. Bear, Dynamics of Fluids in Porous Media. New York: American Elsevier Publishing Company, Inc., 1972.

[3] H. Hanley, Transport Phenomena in Fluids. New York: Marcel Dekker, Inc., 1969.

[4] G. Borgia, R. Brown, P. Fantazzini, J. Gore, P. Mansfield, B. Maraviglia, E. Mesini, and L. Sgubini, eds., Proceedings of the Second International Meeting on Recent Advnaces in MR applications to Porous Media, vol. 9. Elsevier, 1991.

[5] G. Borgia, P. Fantazzini, J. Gore, R. Smith, and J. Strange, eds., Proceedings of the Second International Meeting on Recent Advnaces in MR applications to Porous Media, vol. 12. Elsevier, 1994.

[6] G. Borgia, P. Fantazzini, J. Gore, M. Halse, and J. Strange, eds., Proceedings of the Third International Meeting on Recent Advnaces in MR applications to Porous Media, vol. 14. Elsevier, 1996.

[7] G. Borgia, P. Fantazzini, J. Gore, and J. Strange, eds., Proceedings of the Fourth International Meeting on Recent Advnaces in MR applications to Porous Media, vol. 16. Elsevier, 1998. 
[8] G. Borgia, P. Fantazzini, J. Gore, and J. Strange, eds., Proceedings of the Fifth International Meeting on Recent Advnaces in MR applications to Porous Media, vol. 19. Elsevier, 2001.

[9] G. Borgia, P. Fantazzini, J. Gore, H. M., and J. Strange, eds., Proceedings of the Sixth International Meeting on Recent Advnaces in MR applications to Porous Media, vol. 21. Elsevier, 2003.

[10] P. Fantazzini, J. Gore, and J.-P. Korb, eds., Proceedings of the Seventh International Meeting on Recent Advnaces in MR applications to Porous Media, vol. 23. Elsevier, 2005.

[11] P. Fantazzini and J. Gore, eds., Proceedings of the Eighth International Meeting on Recent Advnaces in MR applications to Porous Media, vol. 25. Elsevier, 2007.

[12] M. Hürlimann, Y.-Q. Song, P. Fantazzini, and V. Bortolotti, eds., Proceedings of the Ninth International Meeting on Recent Advnaces in MR applications to Porous Media, vol. 1081. AIP Conference Proceedings, 2008.

[13] D. Koch and J. Brady, "A nonlocal description of advection diffusion with application to dispersion in porous-media," JOURNAL OF FLUID MECHANICS, vol. 180, pp. 387-403, JUL 1987.

[14] J. Seymour and P. Callaghan, "Generalized approach to NMR analysis of flow and dispersion in porous media," AICHE JOURNAL, vol. 43, pp. 2096-2111, AUG 1997.

[15] B. Manz, P. Alexander, and L. Gladden, "Correlations between dispersion and structure in porous media probed by nuclear magnetic resonance," PHYSICS OF FLUIDS, vol. 11, pp. 259-267, FEB 1999.

[16] A. Khrapitchev and P. Callaghan, "Reversible and irreversible dispersion in a porous medium," PHYSICS OF FLUIDS, vol. 15, pp. 2649-2660, SEP 2003. 
[17] P. Callaghan, "Some perspectives on dispersion and the use of ensemble-averaged PGSE NMR," MAGNETIC RESONANCE IMAGING, vol. 23, pp. 133-137, FEB 2005. 7th International Conference on Magnetic Resonance in Porous Media (MRPM7), Palaiseau, FRANCE, JUL 04-08, 2004.

[18] S. Stapf, "NMR investigations of correlations between longitudinal and transverse displacements in flow through random structured media," CHEMICAL PHYSICS, vol. 284, pp. 369-388, NOV 12002.

[19] S. Stapf, S. Han, C. Heine, and B. Blumich, "Spatiotemporal correlations in transport processes determined by multiple pulsed field gradient experiments," CONCEPTS IN MAGNETIC RESONANCE, vol. 14, no. 3, pp. 172-211, 2002.

[20] U. Tallarek, T. Scheenen, and H. Van As, "Macroscopic heterogeneities in electroosmotic and pressure-driven flow through fixed beds at low column-to-particle diameter ratio," JOURNAL OF PHYSICAL CHEMISTRY B, vol. 105, pp. 8591-8599, SEP 132001.

[21] S. Stapf, K. Packer, S. Bekri, and P. Adler, "Two-dimensional nuclear magnetic resonance measurements and numerical simulations of fluid transport in porous rocks," PHYSICS OF FLUIDS, vol. 12, pp. 566-580, MAR 2000.

[22] B. Manz, L. Gladden, and P. Warren, "Flow and dispersion in porous media: Lattice-Boltzmann and NMR studies," AICHE JOURNAL, vol. 45, pp. 1845-1854, SEP 1999.

[23] S. Stapf, K. Packer, R. Graham, J. Thovert, and P. Adler, "Spatial correlations and dispersion for fluid transport through packed glass beads studied by pulsed field-gradient NMR," PHYSICAL REVIEW E, vol. 58, pp. 6206-6221, NOV 1998. 
[24] A. Ding and D. Candela, "Probing nonlocal tracer dispersion in flows through random porous media," PHYSICAL REVIEW E, vol. 54, pp. 656-660, JUL 1996.

[25] F. A. L. Dullien, Porous Media. Fluid Transport and Pore Structure. Second Edition. New York: Academic Press., 1992.

[26] A. Watson and C. Chang, "Characterizing porous media with NMR methods," PROGRESS IN NUCLEAR MAGNETIC RESONANCE SPECTROSCOPY, vol. 31, pp. 343-386, NOV 1997.

[27] P. Barrie, "Characterization of porous media using NMR methods," in ANNUAL REPORTS ON NMR SPECTROSCOPY, VOL 41, vol. 41 of ANNUAL REPORTS ON NMR SPECTROSCOPY, pp. 265-316, 2000.

[28] P. Danckwerts, "Continuous flow systems - distribution of residence times," CHEMICAL ENGINEERING SCIENCE, vol. 2, no. 1, pp. 1-13, 1953.

[29] U. Scheven, D. Verganelakis, R. Harris, M. Johns, and L. Gladden, "Quantitative nuclear magnetic resonance measurements of preasymptotic dispersion in flow through porous media," PHYSICS OF FLUIDS, vol. 17, NOV 2005.

[30] C. J.H., The Physcis of Fluids in Hierarchical Porous Media: Angstroms to Miles. New Your: Kluwer Academic Publishers, 1997.

[31] N. Morrow, "Small-scale packing heterogeneities in porous sedimentary rocks," AMERICAN ASSOCIATION OF PETROLEUM GEOLOGISTS BULLETIN, vol. 55, no. 3, pp. 514-\&, 1971.

[32] Y. Song, S. Ryu, and P. Sen, "Determining multiple length scales in rocks," NATURE, vol. 406, pp. 178-181, JUL 132000. 
[33] K. Brownstein and C. Tarr, "Importance of classical diffusion in NMR-studies of water in biological cells," PHYSICAL REVIEW A, vol. 19, no. 6, pp. 2446-2453, 1979.

[34] R. Kleinberg, W. Kenyon, and P. Mitra, "Mechanism of NMR relaxation of fluids in rock," JOURNAL OF MAGNETIC RESONANCE SERIES A, vol. 108, pp. 206-214, JUN 1994.

[35] L. Latour, R. Kleinberg, P. Mitra, and C. Sotak, "Pore-size distributions and tortuosity in heterogeneous porous-media," JOURNAL OF MAGNETIC RESONANCE SERIES A, vol. 112, pp. 83-91, JAN 1995.

[36] K. Meyer and P. Klobes, "Comparison between different presentations of pore size distribution in porous materials," FRESENIUS JOURNAL OF ANALYTICAL CHEMISTRY, vol. 363, pp. 174-178, JAN 1999.

[37] C. Arns, "A comparison of pore size distributions derived by NMR and X-ray-CT techniques," PHYSICA A-STATISTICAL MECHANICS AND ITS APPLICATIONS, vol. 339, pp. 159-165, AUG 12004. Conference on New Materials and Complexity, Canberra, AUSTRALIA, NOV 03-07, 2003.

[38] P. Mitra, P. Sen, L. Schwartz, and P. Ledoussal, "Diffusion propagator as a probe of the structure of porous-media," PHYSICAL REVIEW LETTERS, vol. 68, pp. 3555-3558, JUN 151992.

[39] P. Mitra, P. Sen, and L. Schwartz, "Short-time behavior of the diffusion-coefficient as a geometrical probe of porous-media," PHYSICAL REVIEW B, vol. 47, pp. 8565-8574, APR 11993.

[40] H. Liaw, R. Kulkarni, S. Chen, and A. Watson, "Characterization of fluid distributions in porous media by NMR techniques," AICHE JOURNAL, vol. 42, pp. 538-546, FEB 1996. 
[41] S. Bryant and M. Blunt, "Prediction of relative permeability in simple porous-media," PHYSICAL REVIEW A, vol. 46, pp. 2004-2011, AUG 151992.

[42] A. Dixon and M. Nijemeisland, "CFD as a design tool for fixedbed reactors," INDUSTRIAL \& ENGINEERING CHEMISTRY RESEARCH, vol. 40, pp. 5246-5254, NOV 14 2001. 8th Conference on Novel Reactor Engineering for the New Millennium, BARGA, ITALY, JUN 24-29, 2001.

[43] T. Atmakidis and E. Y. Kenig, "CFD-based analysis of the wall effect on the pressure drop in packed beds with moderate tube/particle diameter ratios in the laminar flow regime," CHEMICAL ENGINEERING JOURNAL, vol. 155, pp. 404-410, DEC 12009.

[44] A. Jafari, P. Zamankhan, S. M. Mousavi, and K. Pietarinen, "Modeling and CFD simulation of flow behavior and dispersivity through randomly packed bed reactors," CHEMICAL ENGINEERING JOURNAL, vol. 144, pp. 476-482, NOV 12008.

[45] G. Barton, Elements of Green's Functions and Propagation: Potentials, Diffusion and Waves. New York: Oxford University Press., 1989.

[46] J. Salles, J. Thovert, R. Delannay, L. Prevors, J. Auriault, and P. ADLER, "Taylor dispersion in porous-media - Determination of the dispersion tensor," PHYSICS OF FLUIDS A-FLUID DYNAMICS, vol. 5, pp. 2348-2376, OCT 1993.

[47] P. Sen, "Time-dependent diffusion coefficient as a probe of geometry," CONCEPTS IN MAGNETIC RESONANCE PART A, vol. 23A, pp. 1-21, SEP 2004.

[48] H. Brenner, "Dispersion resulting from flow through spatially periodic porous-media," PHILOSOPHICAL TRANSACTIONS OF THE ROYAL SOCIETY OF LONDON SERIES A-MATHEMATICAL 
PHYSICAL AND ENGINEERING SCIENCES, vol. 297, no. 1430, pp. 81-133, 1980.

[49] C. Lowe and D. Frenkel, "Do hydrodynamic dispersion coefficients exist?," PHYSICAL REVIEW LETTERS, vol. 77, pp. 4552-4555, NOV 251996.

[50] P. T. Callaghan, "Pulsed-gradient spin-echo NMR for planar, cylindrical, and spherical pores under conditions of wall relaxation," $J$. Magn. Reson. A, vol. 113, no. 1, pp. 53 - 59, 1995.

[51] P. Callaghan, A. COY, D. Macgowan, K. Packer, and F. Zelaya, "Diffraction-Like Effects In Nmr Diffusion Studies Of Fluids In Porous Solids," NATURE, vol. 351, pp. 467-469, JUN 61991.

[52] M. Quintard and S. Whitaker, "Transport in ordered and disordered porous-media - Volume-averaged equations, closure problems, and comparison with experiment," CHEMICAL ENGINEERING SCIENCE, vol. 48, pp. 2537-2564, JUL 1993. CHEMICAL ENGINEERING FORUM FOR YOUNG SCIENTISTS, TORINO, ITALY, JUL 02-04, 1992.

[53] J. Seymour and P. Callaghan, "'Flow-diffraction" structural characterization and measurement of hydrodynamic dispersion in porous media by PGSE NMR," JOURNAL OF MAGNETIC RESONANCE SERIES A, vol. 122, pp. 90-93, SEP 1996.

[54] G. Taylor, "Diffusion and mass transport in tubes," PROCEEDINGS OF THE PHYSICAL SOCIETY OF LONDON SECTION B, vol. 67, no. 420, pp. 857-869, 1954.

[55] C. Vandenbroeck, "Taylor dispersion revisited," PHYSICA A, vol. 168, pp. 677-696, OCT 11990. 
[56] K. Ekambara and J. Joshi, "Axial mixing in laminar pipe flows," CHEMICAL ENGINEERING SCIENCE, vol. 59, pp. 3929-3944, SEP 2004.

[57] Y. Zhang, D. A. Benson, M. M. Meerschaert, and E. M. LaBolle, "Space-fractional advection-dispersion equations with variable parameters: Diverse formulas, numerical solutions, and application to the Macrodispersion Experiment site data," WATER RESOURCES RESEARCH, vol. 43, MAY 252007.

[58] E. Sudicky, J. Cherry, and E. Frind, "Migration of contaminants in groundwater at a landfill - A case-study .4. A natural-gradient dispersion-test," JOURNAL OF HYDROLOGY, vol. 63, no. 1-2, pp. 81-108, 1983.

[59] D. Mackay, D. Freyberg, and P. Roberts, "A natural gradient experiment on solute transport in a sand aquifer .1. Approach and overview of plume movement," WATER RESOURCES RESEARCH, vol. 22, pp. 2017-2029, DEC 1986.

[60] L. Gelhar, C. Welty, and K. Rehfeldt, "A critical-review of data on field-scale dispersion in aquifers," WATER RESOURCES RESEARCH, vol. 28, pp. 1955-1974, JUL 1992.

[61] A. Cortis and B. Berkowitz, "Anomalous transport in "classical" soil and sand columns," SOIL SCIENCE SOCIETY OF AMERICA JOURNAL, vol. 68, pp. 1539-1548, SEP-OCT 2004.

[62] M. Levy and B. Berkowitz, "Measurement and analysis of nonFickian dispersion in heterogeneous porous media," JOURNAL OF CONTAMINANT HYDROLOGY, vol. 64, pp. 203-226, JUL 2003.

[63] M. Moroni, N. Kleinfelter, and J. H. Cushman, "Analysis of dispersion in porous media via matched-index particle tracking velocime- 
try experiments," ADVANCES IN WATER RESOURCES, vol. 30, pp. 1-15, JAN 2007.

[64] S. Silliman, L. Konikow, and C. Voss, "Laboratory investigation of longitudinal dispersion in anisotropic porous-media," WATER RESOURCES RESEARCH, vol. 23, pp. 2145-2151, NOV 1987.

[65] S. Sternberg, J. Cushman, and R. Greenkorn, "Laboratory observation of nonlocal dispersion," TRANSPORT IN POROUS MEDIA, vol. 23, pp. 135-151, MAY 1996.

[66] J. Seymour, J. Gage, S. Codd, and R. Gerlach, "Anomalous fluid transport in porous media induced by biofilm growth," PHYSICAL REVIEW LETTERS, vol. 93, NOV 52004.

[67] F. Klammler and R. Kimmich, "Geometrical restrictions of incoherent transport of water by diffusion in protein of silica fineparticle systems and by flow in a sponge - a study of anomalous properties using an NMR field-gradient technique," croatica chemica acta, vol. 65, pp. 455-470, AUG 151992.

[68] S. P. Neuman and D. M. Tartakovsky, "Perspective on theories of non-Fickian transport in heterogeneous media," ADVANCES IN WATER RESOURCES, vol. 32, pp. 670-680, MAY 2009. Fall Meeting of the American-Geophysical-Union, San Francisco, CA, DEC, 2006.

[69] J. Jackson, Classical Electrodynamics - 3rd Edition. New York: American Elsevier Publishing Company, Inc., 1999.

[70] D. G. B. Edelen, Nonlocal field theories, in: Eringen (ed), Continuum Physics. New York: Academic Press, 1976.

[71] J. Cushman and B. Hu, "A resume of nonlocal transport theories," STOCHASTIC HYDROLOGY AND HYDRAULICS, vol. 9, pp. 105116, JUN 1995. 
[72] A. Ding, PFG NMR Study of Hydrodynamic dispersion in porous media. PhD thesis, University of Massachuesettes Amherst, 1997.

[73] P. Callaghan, Principles of Nuclear Magnetic Resonance Microscopy. Oxford University Press, 1991.

[74] B. Blümich, NMR Imaging of Materials. Oxford University Press, 2000

[75] R. R. Ernst, Principles of Nuclear Magnetic Resonance in One and Two Dimensions. Oxford University Press, 1990.

[76] M. Levitt, Spin Dynamics: Basics of Nuclear Magnetic Resonance. John Wiley \& Sons, LTD, 2001.

[77] A. Abragam, Principles of Nuclear Magnetism. Oxford University Press, 1961.

[78] E. Fukushima and S. Roeder, Experimental Pulse NMR and Nuts nad Bolts Approach. Westvire Press, 1981.

[79] H. Trodahl and B. Turrell, "Thermometric detection of nuclear magnetic-resonance," JOURNAL OF LOW TEMPERATURE PHYSICS, vol. 10, no. 1-2, pp. 217-223, 1973.

[80] J. Mispelter, M. Lupu, and A. Briguet, NMR probeheads for Biophysical and Biomedical Experiments. Imperial College Press, 2006.

[81] E. Hahn, "Spin echoes," PHYSICAL REVIEW, vol. 80, no. 4, pp. 580$594,1950$.

[82] P. Callaghan and J. Stepisnik, "Generalised Analysis of Motion using Magnetic Field Gradients," Advances in Magnetic Resonance, vol. 19, pp. 325-388, 1996. 
[83] E. Fukushima, "Nuclear magnetic resonance as a tool to study flow," ANNUAL REVIEW OF FLUID MECHANICS, vol. 31, pp. 95-123, 1999.

[84] Mansfield.P and P. Grannell, "NMR Diffraction In Solids," JOURNAL OF PHYSICS C-SOLID STATE PHYSICS, vol. 6, no. 22, pp. L422-L426, 1973.

[85] E. Stejskal and J. Tanner, "Spin diffusion measurements - spin echoes in presence of a time-dependent field gradient," JOURNAL OF CHEMICAL PHYSICS, vol. 42, no. 1, pp. 288-\&, 1965.

[86] J. Karger and W. Heink, "The propagator representation of molecular-transport in microporous crystallites," JOURNAL OF MAGNETIC RESONANCE, vol. 51, no. 1, pp. 1-7, 1983.

[87] A. Khrapitchev and P. Callaghan, "Double PGSE NMR with stimulated echoes: Phase cycles for the selection of desired encoding," JOURNAL OF MAGNETIC RESONANCE, vol. 152, pp. 259-268, OCT 2001.

[88] P. Callaghan and B. Manz, "Velocity exchange spectroscopy," JOURNAL OF MAGNETIC RESONANCE SERIES A, vol. 106, pp. 260-265, FEB 1994.

[89] R. Cotts, M. Hoch, T. Sun, and J. Markert, "Pulsed field gradient stimulated echo methods for improved NMR diffusion measurements in heterogeneous systems," JOURNAL OF MAGNETIC RESONANCE, vol. 83, pp. 252-266, JUN 151989.

[90] P. Majors, J. Smith, F. kovarik, and E. Fukushima, "NMR spectroscopic imaging of oil displacement in dolomite," JOURNAL OF MAGNETIC RESONANCE, vol. 89, pp. 470-478, OCT 11990. 
[91] G. Guillot, G. Kassab, J. Hulin, and P. Rigord, "Monitoring of tracer dispersion in porous-media by NMR imaging," JOURNAL OF PHYSICS D-APPLIED PHYSICS, vol. 24, pp. 763-773, MAY 141991.

[92] G. Guillot, A. Trokiner, L. Darrasse, A. Dupas, F. Ferdossi, G. KASSAB, J. HULIN, P. RIGORD, and H. SAINTJALMES, “NMR imaging applied to various studies of porous-media," MAGNETIC RESONANCE IMAGING, vol. 9, no. 5, pp. 821-825, 1991. 1ST INTERNATIONAL MEETING ON RECENT ADVANCES IN NMR APPLICATIONS TO POROUS MEDIA, BOLOGNA, ITALY, NOV 14-16, 1990.

[93] L. Lebon, J. Leblond, J. Hulin, N. Martys, and L. Schwartz, "Pulsed field gradient NMR measurements of probability distribution of displacement under flow in sphere packings," MAGNETIC RESONANCE IMAGING, vol. 14, no. 7-8, pp. 989-991, 1996. 3rd International Meeting on Recent Advances in MR Applications to Porous Media, LOUVAIN, BELGIUM, SEP 03-06, 1995.

[94] L. Lebon, L. Oger, J. Leblond, J. Hulin, N. Martys, and L. Schwartz, "Pulsed gradient NMR measurements and numerical simulation of flow velocity distribution in sphere packings," PHYSICS OF FLUIDS, vol. 8, pp. 293-301, FEB 1996.

[95] L. Lebon, J. Leblond, and J. Hulin, “Experimental measurement of dispersion processes at short times using a pulsed field gradient NMR technique," PHYSICS OF FLUIDS, vol. 9, pp. 481-490, MAR 1997.

[96] M. Amin, S. Gibbs, R. Chorley, K. Richards, T. Carpenter, and L. Hall, "Study of flow and hydrodynamic dispersion in a porous medium using pulsed-field-gradient magnetic resonance," PROCEEDINGS OF THE ROYAL SOCIETY OF LONDON SERIES A- 
MATHEMATICAL PHYSICAL AND ENGINEERING SCIENCES, vol. 453, pp. 489-513, MAR 81997.

[97] U. Tallarek, K. Albert, E. Bayer, and G. Guiochon, "Measurement of transverse and axial apparent dispersion coefficients in packed beds," AICHE JOURNAL, vol. 42, pp. 3041-3054, NOV 1996.

[98] S. Codd, B. Manz, J. Seymour, and P. Callaghan, "Taylor dispersion and molecular displacements in Poiseuille flow," PHYSICAL REVIEW E, vol. 60, pp. R3491-R3494, OCT 1999.

[99] U. Tallarek, D. van Dusschoten, H. Van As, E. Bayer, and G. Guiochon, "Study of transport phenomena in chromatographic columns by pulsed field gradient NMR," JOURNAL OF PHYSICAL CHEMISTRY B, vol. 102, pp. 3486-3497, APR 301998.

[100] U. Tallarek, E. Bayer, and G. Guiochon, "Study of dispersion in packed chromatographic columns by pulsed field gradient nuclear magnetic resonance," JOURNAL OF THE AMERICAN CHEMICAL SOCIETY, vol. 120, pp. 1494-1505, FEB 251998.

[101] U. Tallarek, D. van Dusschoten, H. Van As, G. Guiochon, and E. Bayer, "Mass transfer in chromatographic columns studied by PFG NMR," MAGNETIC RESONANCE IMAGING, vol. 16, pp. 699702, JUN-JUL 1998.

[102] A. Sederman, M. Johns, A. Bramley, P. Alexander, and L. Gladden, "Magnetic resonance imaging of liquid flow and pore structure within packed beds," CHEMICAL ENGINEERING SCIENCE, vol. 52, pp. 2239-2250, JUL 1997.

[103] A. Sederman, M. Johns, P. Alexander, and L. Gladden, "Structureflow correlations in packed beds," CHEMICAL ENGINEERING SCIENCE, vol. 53, pp. 2117-2128, JUN 1998. 
[104] X. Ren, S. Stapf, and B. Blumich, "NMR velocimetry of flow in model fixed-bed reactors of low aspect ratio," AICHE JOURNAL, vol. 51, pp. 392-405, FEB 2005.

[105] P. Callaghan, S. Codd, and J. Seymour, "Spatial coherence phenomena arising from translational spin motion in gradient spin echo experiments," CONCEPTS IN MAGNETIC RESONANCE, vol. 11, no. 4, pp. 181-202, 1999.

[106] E. Ozarslan, N. Shemesh, and P. J. Basser, "A general framework to quantify the effect of restricted diffusion on the NMR signal with applications to double pulsed field gradient NMR experiments," JOURNAL OF CHEMICAL PHYSICS, vol. 130, MAR 142009.

[107] N. Shemesh, E. Oezarslan, A. Bar-Shir, P. J. Basser, and Y. Cohen, "Observation of restricted diffusion in the presence of a free diffusion compartment: Single- and double-PFG experiments," JOURNAL OF MAGNETIC RESONANCE, vol. 200, pp. 214-225, OCT 2009.

[108] N. Shemesh, E. Ozarslan, P. J. Basser, and Y. Cohen, "Detecting diffusion-diffraction patterns in size distribution phantoms using double-pulsed field gradient NMR: Theory and experiments," JOURNAL OF CHEMICAL PHYSICS, vol. 132, JAN 212010.

[109] P. Callaghan and J. Stepisnik, "Frequency-domain analysis of spin motion using modulated-gradient NMR," JOURNAL OF MAGNETIC RESONANCE SERIES A, vol. 117, pp. 118-122, NOV 1995.

[110] P. Callaghan and S. Codd, "Flow coherence in a bead pack observed using frequency domain modulated gradient nuclear magnetic resonance," PHYSICS OF FLUIDS, vol. 13, pp. 421-427, FEB 2001.

[111] J. Stepisnik, A. Mohoric, and A. Duh, "Diffusion and flow in a 
porous structure by the gradient spin echo spectral analysis," PHYSICA B-CONDENSED MATTER, vol. 307, pp. 158-168, DEC 2001.

[112] E. Parsons, M. Does, and J. Gore, "Modified oscillating gradient pulses for direct sampling of the diffusion spectrum suitable for imaging sequences," MAGNETIC RESONANCE IMAGING, vol. 21, pp. 279-285, APR-MAY 2003. 6th International Conference on the Recent Advances in Magnetic Resonance Applications to Porous Media, ULM, GERMANY, SEP 08-12, 2002.

[113] U. Scheven and P. Sen, "Spatial and temporal coarse graining for dispersion in randomly packed spheres," PHYSICAL REVIEW LETTERS, vol. 89, DEC 162002.

[114] U. Scheven, J. Seland, and D. Cory, "NMR propagator measurements on flow through a random pack of porous glass beads and how they are affected by dispersion, relaxation, and internal field inhomogeneities," PHYSICAL REVIEW E, vol. 69, FEB 2004.

[115] M. Britton, R. Graham, and K. Packer, "Relationships between flow and NMR relaxation of fluids in porous solids," MAGNETIC RESONANCE IMAGING, vol. 19, pp. 325-331, APR-MAY 2001. 5th International Meeting on Recent Advances in Magnetic Resonance Applications to Porous Media, BOLOGNA, ITALY, OCT 09-11, 2000.

[116] J. Mitchell, D. A. G. von der Schulenburg, D. J. Holland, E. J. Fordham, M. L. Johns, and L. F. Gladden, "Determining NMR flow propagator moments in porous rocks without the influence of relaxation," JOURNAL OF MAGNETIC RESONANCE, vol. 193, pp. 218225, AUG 2008.

[117] S. Codd and S. Altobelli, "A PGSE study of propane gas flow through model porous bead packs," JOURNAL OF MAGNETIC RESONANCE, vol. 163, pp. 16-22, JUL 2003. 
[118] I. Koptyug, A. Matveev, and S. Altobelli, "NMR studies of hydrocarbon gas flow and dispersion," APPLIED MAGNETIC RESONANCE, vol. 22, no. 2, pp. 187-200, 2002. 6th International Conference on Magnetic Resonance Microscopy, NOTTINGHAM, ENGLAND, SEP, 2001.

[119] J. Granwehr, E. Harel, C. Hilty, S. Garcia, L. Chavez, A. Pines, P. N. Sen, and Y.-Q. Song, "Dispersion measurements using time-offlight remote detection MRI," MAGNETIC RESONANCE IMAGING, vol. 25, pp. 449-452, MAY 2007. 8th International Bologna Conferene on Magnetic Resonance in Porous Media, Bologna, ITALY, SEP $10,2006$.

[120] L. Kaiser, J. Logan, T. Meersmann, and A. Pines, "Dynamic NMR microscopy of gas phase Poiseuille flow," JOURNAL OF MAGNETIC RESONANCE, vol. 149, pp. 144-148, MAR 2001.

[121] B. S. Akpa, D. J. Holland, A. J. Sederman, M. L. Johns, and L. F. Gladden, "Enhanced C-13 PFG NMR for the study of hydrodynamic dispersion in porous media," JOURNAL OF MAGNETIC RESONANCE, vol. 186, pp. 160-165, MAY 2007.

[122] J. Stamps, B. Ottink, J. Visser, J. van Duynhoven, and R. Hulst, “Difftrain: A novel approach to a true spectroscopic single-scan diffusion measurement," JOURNAL OF MAGNETIC RESONANCE, vol. 151, pp. 28-31, JUL 2001.

[123] J. Mitchell, A. Sederman, E. J. Fordham, M. L. Johns, and L. F. Gladden, "A rapid measurement of flow propagators in porous rocks," JOURNAL OF MAGNETIC RESONANCE, vol. 191, pp. 267-272, APR 2008.

[124] S. Succi, The lattice Boltzmann equation for fluid dynamics and beyond. Oxford University Press, 2001. 
[125] S. Chen and G. Doolen, "Lattice Boltzmann method for fluid flows," ANNUAL REVIEW OF FLUID MECHANICS, vol. 30, pp. 329-364, 1998.

[126] C. Pan, L.-S. Luo, and C. T. Miller, "An evaluation of lattice Boltzmann schemes for porous medium flow simulation," COMPUTERS E FLUIDS, vol. 35, pp. 898-909, SEP-NOV 2006. 1st International Conference for Mesoscopic Methods in Engineering and Science (ICMMES), Braunschweig, GERMANY, JUL 25, 2004-JUL 30, 2005.

[127] R. S. Maier and R. S. Bernard, "Lattice-Boltzmann accuracy in pore-scale flow simulation," JOURNAL OF COMPUTATIONAL PHYSICS, vol. 229, pp. 233-255, JAN 202010.

[128] S. Sullivan, F. Sani, M. Johns, and L. Gladden, "Simulation of packed bed reactors using lattice Boltzmann methods," CHEMICAL ENGINEERING SCIENCE, vol. 60, pp. 3405-3418, JUN 2005.

[129] S. Succi, E. Foti, and F. Higuera, "3-dimensional flows in complex geometries with the lattice Boltzmann method," EUROPHYSICS LETTERS, vol. 10, pp. 433-438, NOV 11989.

[130] G. Mcnamara and G. Zanetti, "Use of the Boltzmann-equation to simulate lattice-gas automata," PHYSICAL REVIEW LETTERS, vol. 61, pp. 2332-2335, NOV 141988.

[131] F. Higuera and J. Jimenez, "Boltzmann approach to lattice gas simulations," EUROPHYSICS LETTERS, vol. 9, pp. 663-668, AUG 11989.

[132] U. Frisch, B. Hasslacher, and Y. Pomeau, "Lattice-gas automata for the Navier-Stokes equation," PHYSICAL REVIEW LETTERS, vol. 56, pp. 1505-1508, APR 71986.

[133] X. He and L. Luo, "Theory of the lattice Boltzmann method: From the Boltzmann equation to the lattice Boltzmann equation," PHYSICAL REVIEW E, vol. 56, pp. 6811-6817, DEC 1997. 
[134] X. He and L. Luo, "A priori derivation of the lattice Boltzmann equation," PHYSICAL REVIEW E, vol. 55, pp. R6333-R6336, JUN 1997.

[135] R. Maier, D. Kroll, Y. Kutsovsky, H. Davis, and R. Bernard, "Simulation of flow through bead packs using the lattice Boltzmann method," PHYSICS OF FLUIDS, vol. 10, pp. 60-74, JAN 1998.

[136] J. D. Brewster, "Lattice-Boltzmann simulations of three-dimensional fluid flow on a desktop computer," ANALYTICAL CHEMISTRY, vol. 79, pp. 2965-2971, APR 12007.

[137] P. Bhatnagar, E. Gross, and M. Krook, "A model for collision processes in gases .1. Small amplitude processes in charged and neutral one-component systems," PHYSICAL REVIEW, vol. 94, no. 3, pp. 511-525, 1954.

[138] S. Chen, H. Chen, D. Martinez, and W. Matthaeus, "Lattice Boltzmann model for simulation of magnetohydrodynamics," PHYSICAL REVIEW LETTERS, vol. 67, pp. 3776-3779, DEC 301991.

[139] H. Chen, S. Chen, and W. Matthaeus, "Recovery of the NavierStokes equations using a lattice-gas Boltzmann method," PHYSICAL REVIEW A, vol. 45, pp. R5339-R5342, APR 151992.

[140] P. Adler, M. Zuzovsky, and H. Brenner, "Spatially periodic suspensions of convex particles in linear shear flows," INTERNATIONAL JOURNAL OF MULTIPHASE FLOW, vol. 11, no. 3, pp. 387-417, 1985.

[141] D. ZIEGLER, “BOUNDARY-CONDITIONS FOR LATTICE BOLTZMANN SIMULATIONS," JOURNAL OF STATISTICAL PHYSICS, vol. 71, pp. 1171-1177, JUN 1993.

[142] W. Visscher and M. Bolsterl, "Random packing of equal and unequal spheres in 2 and 3 dimensions," NATURE, vol. 239, no. 5374, pp. 504-\&, 1972. 
[143] R. Jullien, A. Pavlovitch, and P. Meakin, "Random packings of spheres built with sequential models," JOURNAL OF PHYSICS AMATHEMATICAL AND GENERAL, vol. 25, pp. 4103-4113, AUG 7 1992.

[144] A. N. Jackson, H. M. W., and C. P. T., "Simulating dispersion in porous media," to be published, 2010.

[145] P. Szymczak and A. Ladd, "Boundary conditions for stochastic solutions of the convection-diffusion equation," PHYSICAL REVIEW E, vol. 68, SEP 2003.

[146] R. Maier, D. Kroll, R. Bernard, S. Howington, J. Peters, and H. Davis, "Pore-scale simulation of dispersion," PHYSICS OF FLUIDS, vol. 12, pp. 2065-2079, AUG 2000.

[147] F. Jimenez-Hornero, J. Giraldez, and A. Laguna, "Simulation of tracer dispersion in porous media using lattice Boltzmann and random walk models," VADOSE ZONE JOURNAL, vol. 4, pp. 310-316, APR 2005. Vadose Zone Research Meeting, Valladolid, SPAIN, NOV, 2003.

[148] R. Maier, D. Kroll, R. Bernard, S. Howington, J. Peters, and H. Davis, "Enhanced dispersion in cylindrical packed beds," PHILOSOPHICAL TRANSACTIONS OF THE ROYAL SOCIETY OF LONDON SERIES A-MATHEMATICAL PHYSICAL AND ENGINEERING SCIENCES, vol. 360, pp. 497-506, MAR 152002.

[149] R. S. Maier, M. R. Schure, J. P. Gage, and J. D. Seymour, "Sensitivity of pore-scale dispersion to the construction of random bead packs," WATER RESOURCES RESEARCH, vol. 44, JUN 42008.

[150] P. Adler, C. Jacquin, and J. Quiblier, "Flow in simulated porousmedia," INTERNATIONAL JOURNAL OF MULTIPHASE FLOW, vol. 16, pp. 691-712, JUL-AUG 1990. 
[151] J. Tessier and K. Packer, “The characterization of multiphase fluid transport in a porous solid by pulsed gradient stimulated echo nuclear magnetic resonance," PHYSICS OF FLUIDS, vol. 10, pp. 75-85, JAN 1998.

[152] R. Damion, K. Packer, K. Sorbie, and S. McDougall, "Pore-scale network modelling of flow propagators derived from pulsed magnetic field gradient spin echo NMR measurements in porous media," CHEMICAL ENGINEERING SCIENCE, vol. 55, pp. 5981-5998, DEC 2000.

[153] M. Mantle, A. Sederman, and L. Gladden, "Single- and two-phase flow in fixed-bed reactors: MRI flow visualisation and latticeBoltzmann simulations," CHEMICAL ENGINEERING SCIENCE, vol. 56, pp. 523-529, JAN 2001. 16th International Symposium on Chemical Reaction Engineering, KRAKOW, POLAND, SEP 10-13, 2000.

[154] S. Sullivan, L. Gladden, and M. Johns, "Simulation of power-law fluid flow through porous media using lattice Boltzmann techniques," JOURNAL OF NON-NEWTONIAN FLUID MECHANICS, vol. 133, pp. 91-98, FEB 12006.

[155] S. P. Sullivan, A. J. Sederman, M. L. Johns, and L. F. Gladden, "Verification of shear-thinning LB simulations in complex geometries," JOURNAL OF NON-NEWTONIAN FLUID MECHANICS, vol. 143, pp. 59-63, MAY 182007.

[156] A. Ladd, "Numerical simulations of particulate suspensions via a discretized Boltzmann-equation .1. Theoretical foundation," JOURNAL OF FLUID MECHANICS, vol. 271, pp. 285-309, JUL 251994.

[157] S. A. Creber, T. R. R. Pintelon, and M. L. Johns, "Quantification of the velocity acceleration factor for colloidal transport in porous media 
using NMR," JOURNAL OF COLLOID AND INTERFACE SCIENCE, vol. 339, pp. 168-174, NOV 12009.

[158] M. W. Hunter, A. N. Jackson, and P. T. Callaghan, "Nuclear magnetic resonance measurement and lattice-Boltzmann simulation of the nonlocal dispersion tensor," PHYSICS OF FLUIDS, vol. 22, FEB 2010 .

[159] G. Uhlenbeck and L. Ornstein, "On the theory of the Brownian motion," PHYSICAL REVIEW, vol. 36, pp. 0823-0841, SEP 1930.

[160] P. Callaghan, D. Macgowan, K. Packer, and F. Zelaya, "Highresolution q-space imaging in porous structures," JOURNAL OF MAGNETIC RESONANCE, vol. 90, pp. 177-182, OCT 151990.

[161] P. Callaghan, A. Coy, T. Halpin, D. Macgowan, K. Packer, and F. Zelaya, "Diffusion in porous systems and the influence of pore morphology in pulsed gradient spin-echo nuclear-magnetic-resonance studies," JOURNAL OF CHEMICAL PHYSICS, vol. 97, pp. 651-662, JUL 11992.

[162] B. S. Akpa, S. M. Matthews, A. J. Sederman, K. Yunus, A. C. Fisher, M. L. Johns, and L. F. Gladden, "Study of miscible and immiscible flows in a microchannel using magnetic resonance imaging," $A N A$ LYTICAL CHEMISTRY, vol. 79, pp. 6128-6134, AUG 152007.

[163] R. Brown, R. Chandler, J. Jackson, R. Kleinberg, M. Miller, Z. Paltiel, and M. Prammer, "History of NMR well logging," CONCEPTS IN MAGNETIC RESONANCE, vol. 13, no. 6, pp. 335-413, 2001.

[164] G. R. Coats, L. Xiao, and M. G. Prammer, NMR-Logging. Principles and Applications. Gulf Professional Publishing, 2001.

[165] R. Kleinberg, A. Sezginer, D. Griffin, and M. Fukuhara, "Novel NMR apparatus for investigating an external sample," JOURNAL OF MAGNETIC RESONANCE, vol. 97, pp. 466-485, MAY 1992. 
[166] Z. Taicher, G. Coates, Y. Gitartz, and L. Berman, “A comprehensive approach to studies of porous-media (rocks) using a laboratory spectrometer and logging tool with similar operating characteristics," MAGNETIC RESONANCE IMAGING, vol. 12, no. 2, pp. 285-289, 1994. 2nd International Meeting on Recent Advances in MR Applications to Porous Media, CANTERBURY, ENGLAND, APR 14-16, 1993.

[167] M. Peyron, G. Pierens, A. Lucas, L. Hall, and R. Stewart, “The modified stretched-exponential model for characterization of NMR relaxation in porous media," JOURNAL OF MAGNETIC RESONANCE SERIES A, vol. 118, pp. 214-220, FEB 1996.

[168] Y. Song, L. Venkataramanan, M. Hurlimann, M. Flaum, P. Frulla, and C. Straley, "T-1-T-2 correlation spectra obtained using a fast twodimensional Laplace inversion," JOURNAL OF MAGNETIC RESONANCE, vol. 154, pp. 261-268, FEB 2002.

[169] M. Hurlimann, "Diffusion and relaxation effects in general stray field NMR experiments," JOURNAL OF MAGNETIC RESONANCE, vol. 148, pp. 367-378, FEB 2001.

[170] F. Stallmach and J. Karger, "The potentials of pulsed field gradient NMR for investigation of porous media," ADSORPTIONJOURNAL OF THE INTERNATIONAL ADSORPTION SOCIETY, vol. 5, pp. 117-133, MAR 1999.

[171] P. Mansfield and B. Issa, "Fluid transport in porous rocks .1. EPI studies and a stochastic model of flow," JOURNAL OF MAGNETIC RESONANCE SERIES A, vol. 122, pp. 137-148, OCT 1996.

[172] E. Fordham, S. Gibbs, and L. Hall, "Partially restricted diffusion in a permeable sandstone - observations by stimulated echo PFG NMR," MAGNETIC RESONANCE IMAGING, vol. 12, no. 2, pp. 279-284, 
1994. 2nd International Meeting on Recent Advances in MR Applications to Porous Media, CANTERBURY, ENGLAND, APR 14-16, 1993.

[173] M. Hurlimann, "Effective gradients in porous media due to susceptibility differences," JOURNAL OF MAGNETIC RESONANCE, vol. 131, pp. 232-240, APR 1998.

[174] P. Sun, J. Seland, and D. Cory, "Background gradient suppression in pulsed gradient stimulated echo measurements," JOURNAL OF MAGNETIC RESONANCE, vol. 161, pp. 168-173, APR 2003.

[175] R. Waggoner and E. Fukushima, "Velocity distribution of slow fluid flows in Bentheimer sandstone: An NMRI and propagator study," MAGNETIC RESONANCE IMAGING, vol. 14, no. 9, pp. 1085-1091, 1996.

[176] C. Chang and A. Watson, "NMR imaging of flow velocity in porous media," AICHE JOURNAL, vol. 45, pp. 437-444, MAR 1999.

[177] M. Johns, A. Sederman, L. Gladden, A. Wilson, and S. Davies, "Using MR techniques to probe permeability reduction in rock cores," AICHE JOURNAL, vol. 49, pp. 1076-1084, MAY 2003.

[178] P. M. Singer, G. Leu, E. J. Fordham, and P. N. Sen, "Low magnetic fields for flow propagators in permeable rocks," JOURNAL OF MAGNETIC RESONANCE, vol. 183, pp. 167-177, DEC 2006.

[179] D. Verganelakis, J. Crawshaw, M. Johns, M. Mantle, U. Scheven, A. Sederman, and L. Gladden, "Displacement propagators of brine flowing within different types of sedimentary rock," MAGNETIC RESONANCE IMAGING, vol. 23, pp. 349-351, FEB 2005. 7th International Conference on Magnetic Resonance in Porous Media (MRPM7), Palaiseau, FRANCE, JUL 04-08, 2004. 
[180] M. Hunter, A. Jackson, and P. Callaghan, "PGSE NMR measurement of the non-local dispersion tensor for flow in porous media," Journal of Magnetic Resonance, vol. 204, no. 1, pp. 11 - 20, 2010.

[181] M. W. Hunter and P. T. Callaghan, "NMR measurement of nonlocal dispersion in complex flows," PHYSICAL REVIEW LETTERS, vol. 99, NOV 232007.

[182] A. Jerschow and R. Kumar, "Calculation of coherence pathway selection and cogwheel cycles," JOURNAL OF MAGNETIC RESONANCE, vol. 160, pp. 59-64, JAN 2003.

[183] P. Galvosas, F. Stallmach, and J. Karger, "Background gradient suppression in stimulated echo NMR diffusion studies using magic pulsed field gradient ratios," JOURNAL OF MAGNETIC RESONANCE, vol. 166, pp. 164-173, FEB 2004.

[184] J. R. Brown, E. O. Fridjonsson, J. D. Seymour, and S. L. Codd, “Nuclear magnetic resonance measurement of shear-induced particle migration in Brownian suspensions," PHYSICS OF FLUIDS, vol. 21, SEP 2009. 